\title{
The role of STAT1-cooperative DNA binding in myocardial infarction
}

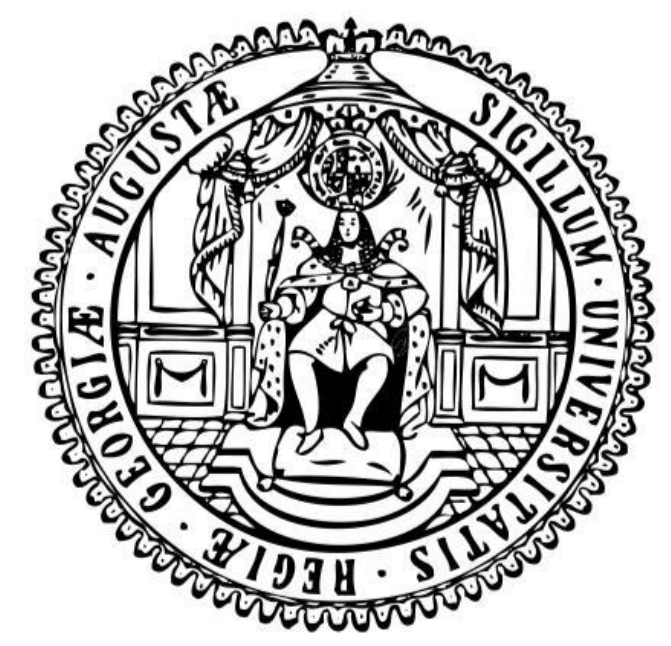

Dissertation

In partial fulfilment of the requirements for the degree

"Doctor rerum naturalium (Dr. rer. nat.)"

of the Georg-August-Universität Göttingen

within the "Molecular Medicine" study program

of the Georg-August University School of Science (GAUSS)

submitted by

Asmma Doudin

born in Amman

Göttingen 2019 


\section{Members of the thesis committee:}

Prof. Dr. mult. Thomas Meyer

Email: $\quad$ thomas.meyer@med.uni-goettingen.de

Postal address: $\quad$ Department of Psychosomatic Medicine and Psychotherapy University Medical Centre Göttingen Georg-August-University Göttingen Waldweg 33 37073 Göttingen

Dr. Aleksandar Ivetic

Email: $\quad$ aleksandar.ivetic@kcl.ac.uk

Postal address: $\quad$ School of Cardiovascular Medicine \& Sciences BHF Centre of Research Excellence James Black Centre King's College London 125, Coldharbour Lane London SE5 9NU

Prof. Dr. rer. nat. Susanne Lutz (second member of the thesis committee)

Email: $\quad$ susanne.lutz@med.uni-goettingen.de

Postal address: $\quad$ Institute of Pharmacology and Toxicology University Medical Centre Göttingen Georg-August-University Göttingen Robert-Koch-Straße 40 37075 Göttingen

Prof. Dr. med. Dörthe Katschinski (third member of the thesis committee)

Email: doerthe.katschinski@med.uni-goettingen.de

Postal address: $\quad$ Institute of Cardiovascular Physiology University Medical Centre Göttingen Georg-August-University Göttingen Humboldtallee 23 37073 Göttingen 


\section{AFFIDAVIT}

Here I declare that my doctoral thesis entitled

"The role of STAT1-cooperative DNA binding in myocardial infarction"

has been written independently with no other sources and aids than quoted.

Asmma Doudin

Göttingen, June 2019 


\section{List of publications}

Doudin A, Becker A, Rothenberger A, Meyer T. Relationship between serum 25hydroxyvitamin D and red blood cell indices in German adolescents. Eur J Pediatr 2018;177(4):583-591.

Doudin A, Becker A, Rothenberger A, Meyer T. The associations between serum 25hydroxyvitamin D and red blood cell indices reported from the KiGGS study are, except for mean corpuscular hemoglobin, not mediated by health-related quality of life. Eur J Pediatr 2018;177(11):1733-1734. 


\section{Table of Contents}

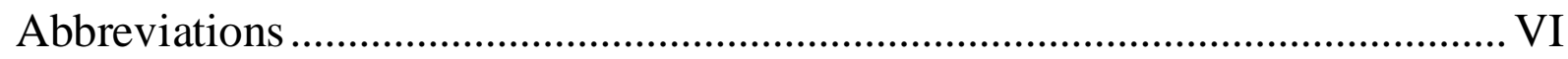

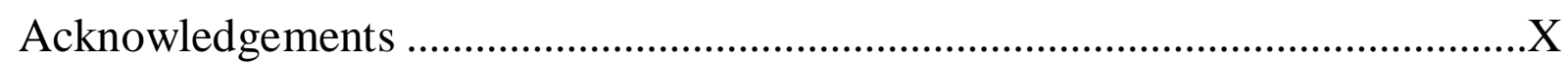

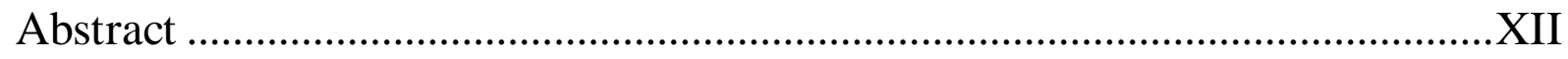

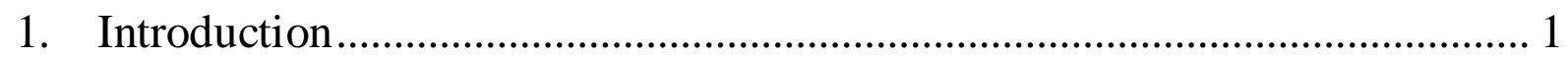

1.1 Discovery of the JAK/STAT signal transduction pathway............................................. 2

1.1.1 Interferons are categorized as cytokines ............................................................. 2

1.1.2 Structure, function, and regulation of STAT proteins .............................................. 3

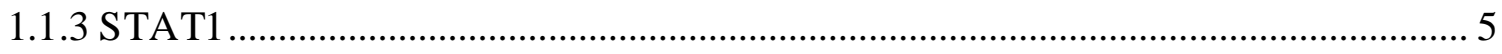

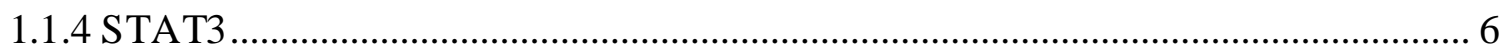

1.1.5 Type I and II interferon signalling ................................................................... 6

1.1.6 STAT1-cooperative DNA binding ………………………………………….... 8

1.1.7 Antagonistic activities of STAT1 and STAT3 ………………………................ 9

1.2 The JAK/STAT signalling pathway in cardiovascular diseases ....................................... 10

1.2.1 Myocardial infarction and leukocytes responses..................................................... 11

1.2.2 Regulation of leucocyte transendothelial migration by the JAK/STAT pathway ... 12

1.3 Original hypotheses and aims of this thesis ............................................................. 13

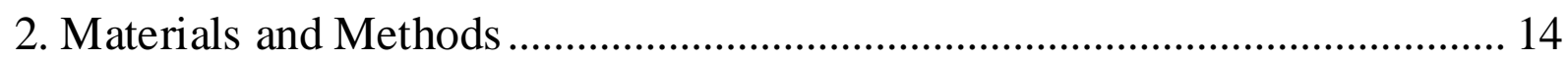

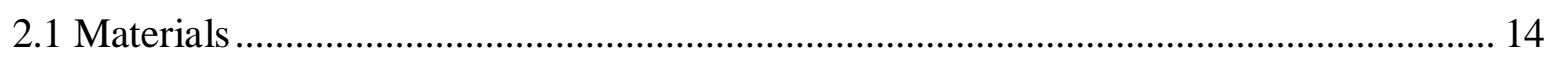

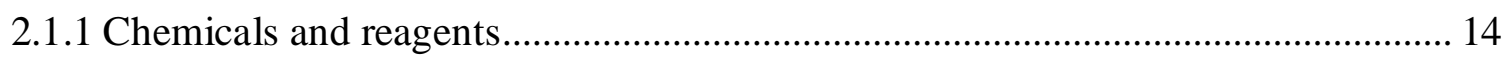

2.1.2 Recombinant proteins and enzymes.................................................................... 17

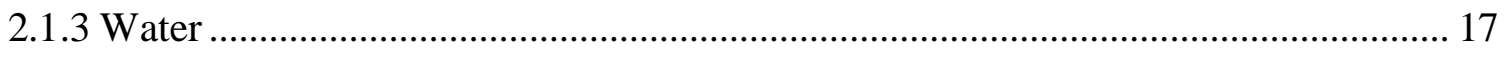

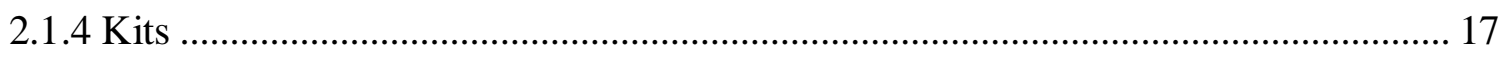

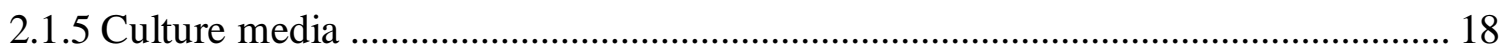

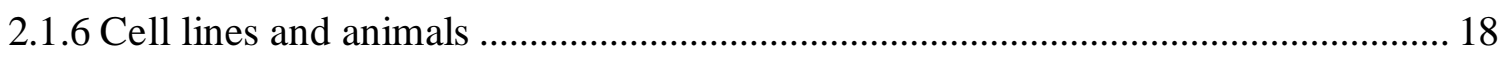

2.1.7 shRNA lentiviral transduction particles .................................................................. 19

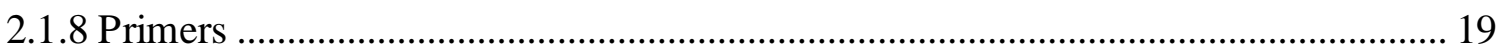

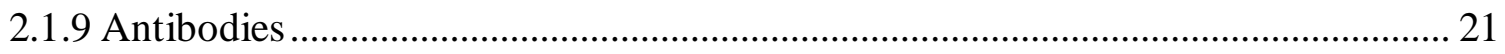

2.1.10 Equipments …………………………………………………………….... 21

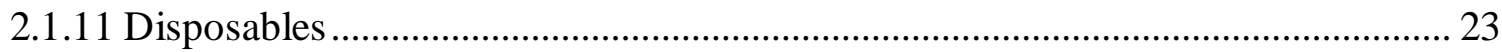




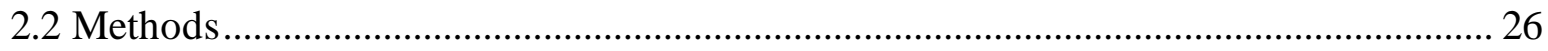

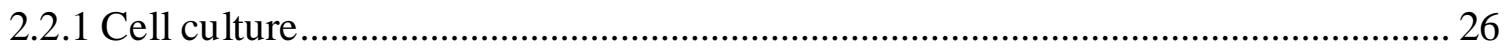

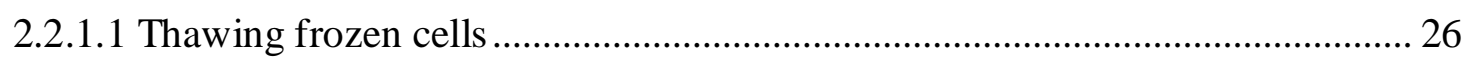

2.2.1.2 Harvesting of cells for cryopreservation ......................................................... 26

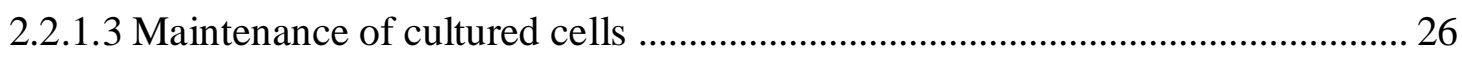

2.2.1.4 Treatment of cultured cells with different stimuli ............................................. 27

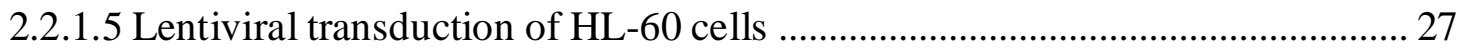

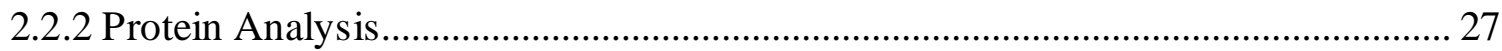

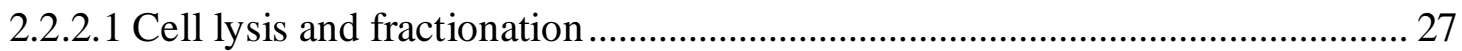

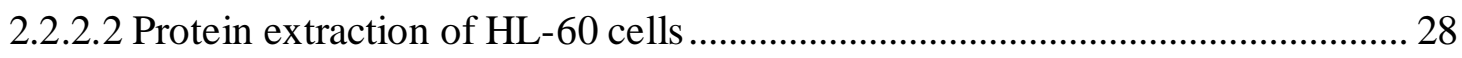

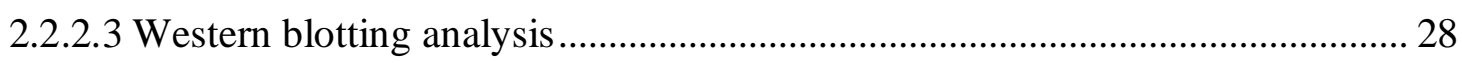

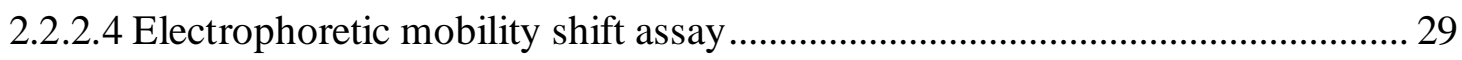

2.2.2.5 Radioactive labelling of EMSA probes ……....................................................... 29

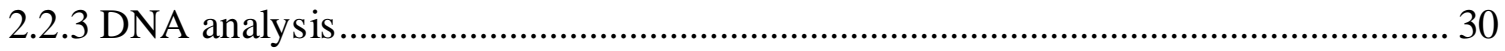

2.2.3.1 DNA isolation from mouse tails ....................................................................... 30

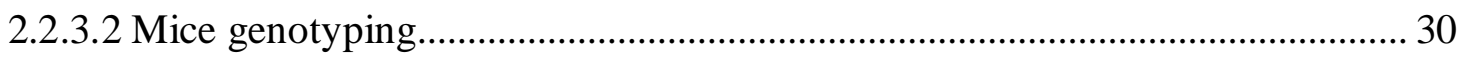

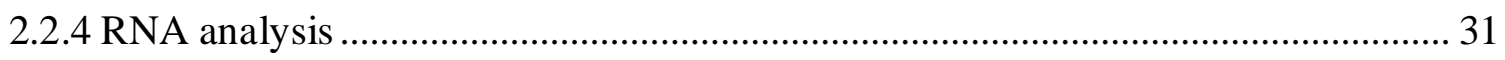

2.2.4.1 RNA extraction from cell culture and reverse transcription ............................. 31

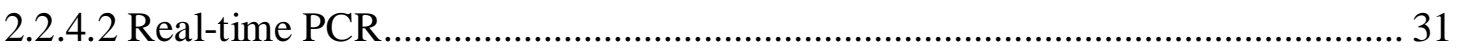

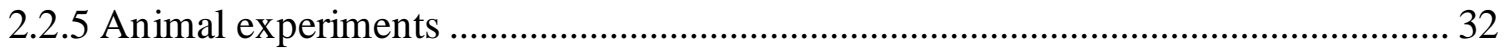

2.2.5.1 Left anterior descending coronary artery ligation in mice .............................. 32

2.2.5.2 Immunohistochemistry of paraffin-embedded sections................................... 32

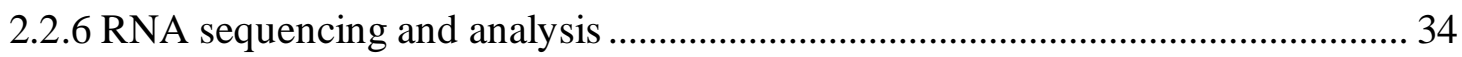

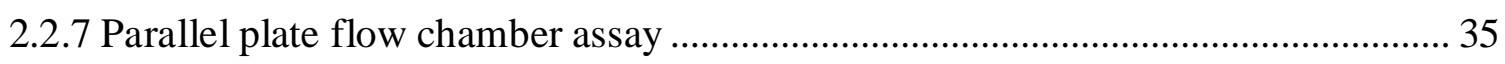

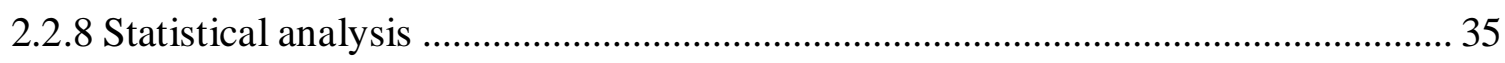

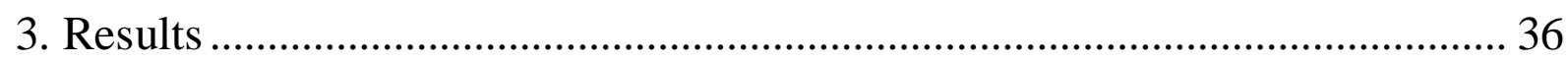

3.1 Echocardiographic analysis demonstrated beneficial outcomes in STAT1-F77A mice

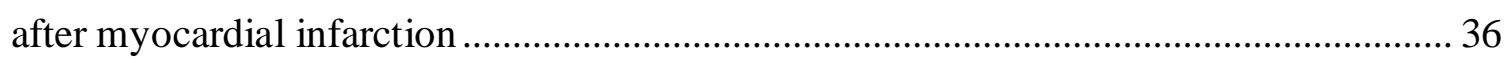

3.2 Expression of STAT1 and STAT3 in both cardiomyocytes and infiltrating immune

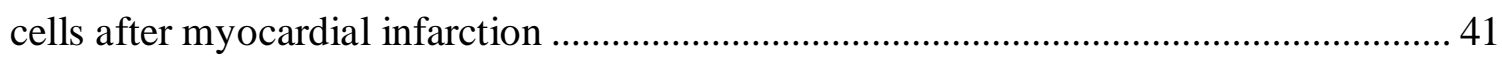

3.3 Transcriptomic analysis of F77A mice in a LAD ligation model ............................... 42 
3.4 Identification of differentially expressed genes in STAT1-F77A and wild-type mice at day 1 post-myocardial infarction

3.5 Gene set enrichment analysis identified common and unique pathways associated with myocardial infarction

3.6 Identification of immune-related pathways differentially regulated after myocardial infarction

3.7 Identification of signal pathways and cellular processes differentially regulated after

LAD ligation 65

3.8 Gene set enrichment analysis revealed changes in metabolic signature following myocardial infarction.

3.9 Differentially regulated pathways in F77A as compared to wild-type mice in the infracted area following LAD ligation

3.10 A gene expression signature indicative of immune cells infiltration detected in F77A mice following LAD ligation 86

3.11 Analysis of STAT1-target genes associated with myocardial infarction. 90

3.12 Co-stimulatory effects of interferon- $\gamma$, interferon- $\alpha$, and interleukin- 6 in MHEC-5 cells

3.13 Generation of STAT1- and STAT3-knockdown HL-60 cell lines

3.14 DMSO-induced differentiation results in upregulation of STAT1 and STAT3 in HL60 cells

3.15 Decreased phosphorylation levels of STAT1 and STAT3 in HL-60 knockdown cells

3.16 Downregulation of ezrin in STAT1- and STAT3-knockdown HL-60 cells 97

3.17 Reduced transendothelial migration in HL-60 cells with decreased STAT1 and STAT3 expression

4. Discussion

5. References 


\section{Abbreviations}

\begin{tabular}{|c|c|}
\hline A & Adenine \\
\hline APS & Ammonium persulfate \\
\hline ATP & Adenosine triphosphate \\
\hline BSA & Bovine serum albumin \\
\hline $\mathrm{C}$ & Cytosine \\
\hline CAMs & Cell adhesion molecules \\
\hline $\mathrm{CBP} / \mathrm{p} 300$ & CREB-binding protein \\
\hline $\mathrm{CCL}$ & $\mathrm{C}-\mathrm{C}$ motif chemokine ligand \\
\hline $\mathrm{CCR}$ & C-C chemokine receptor \\
\hline $\mathrm{CD}$ & Cluster of differentiation \\
\hline cDNA & Complementary deoxyribonucleic acid \\
\hline ChIP & Chromatin immunoprecipitation \\
\hline $\mathrm{CXC}$ & $\mathrm{C}-\mathrm{X}-\mathrm{C}$ motif chemokine \\
\hline CXCL & $\mathrm{C}-\mathrm{X}-\mathrm{C}$ motif chemokine ligand \\
\hline CXCR & CXC chemokine receptor \\
\hline DAB & 3,3'-Diaminobenzidine \\
\hline DBD & DNA-binding domain \\
\hline DMEM & Dulbecco's modified Eagle medium \\
\hline DMSO & Dimethyl sulfoxide \\
\hline DNA & Deoxyribonucleic acid \\
\hline dNTP & Deoxyribonucleotide triphosphate \\
\hline dsDNA & double stranded deoxyribonucleic acid \\
\hline DTT & Dithiothreitol \\
\hline EDTA & Ethylenediaminetetraacetic acid \\
\hline $\mathrm{EF}$ & Ejection fraction \\
\hline EGTA & $\begin{array}{l}\text { Ethylene glycol-bis( } \beta \text {-amimoethyl ether)-N,N,N',N'-tetraacetic } \\
\text { acid }\end{array}$ \\
\hline EMSA & Electrophoretic mobility shift assay \\
\hline ERM & Ezrin, radixin, moesin \\
\hline FAS & Fractional area shortening \\
\hline FBS & Fetal bovine serum \\
\hline FS & Fractional shortening \\
\hline
\end{tabular}




\begin{tabular}{|c|c|}
\hline G & Guanine \\
\hline GAF & Gamma-activating factor \\
\hline GAGE & Generally applicable gene set enrichment \\
\hline GAPDH & Glyceraldehyde-3-phosphate dehydrogenase \\
\hline GBPB & Glucan-binding protein $\mathrm{B}$ \\
\hline GSEA & Gene set enrichment analysis \\
\hline HEPES & 4-(2-Hydroxyethyl)-1-piperazineethanesulfonic acid \\
\hline HIF & Hypoxia-inducible factor \\
\hline HPRT & Hypoxanthine-guanine phosphoribosyltransferase \\
\hline HSP & Heat shock protein \\
\hline HUVEC & Human umbilical vein endothelial cell \\
\hline ICAM & Intercellu lar adhesion molecule \\
\hline IFIT1 & Interferon-induced protein with tetratricopeptide repeats 1 \\
\hline IFN & Interferon \\
\hline IFNAR & Interferon- $\alpha / \beta$ receptor \\
\hline IFNGR & Interferon- $\gamma$ receptor \\
\hline $\operatorname{IgE}$ & Immunoglobulin E \\
\hline $\operatorname{IgG}$ & Immunoglobulin $\mathrm{G}$ \\
\hline IL & Interleukin \\
\hline IL-R & Interleukin receptor \\
\hline IRDYE & Infrared fluorescent dyes \\
\hline IRF & Interferon-regulatory factor \\
\hline ISGF & Interferon-stimulated gene factor \\
\hline ISRE & Interferon-stimulated response element \\
\hline JAKs & Janus kinases \\
\hline $\mathrm{kDa}$ & kilodalton \\
\hline KEGG & Kyoto encyclopedia of genes and genomes \\
\hline LAD & Left anterior descending coronary artery \\
\hline LAVES & Landesamt für Verbraucherschutz und Lebensmittelsicherheit \\
\hline $\log$ & Logarithm \\
\hline LV & Left ventricle \\
\hline $\mathrm{LVID}_{\mathrm{D}}$ & Left ventricular internal dimension-diastole \\
\hline LVIDs & Left ventricular internal dimension-systole \\
\hline MAPK & Mitogen-activated protein kinase \\
\hline
\end{tabular}




\begin{tabular}{|c|c|}
\hline MCP-1 & Monocyte-chemoattractant protein-1 \\
\hline MHEC5-T & Mouse heart endothelial cell clone 5-transformed \\
\hline MI & Myocardial infarction \\
\hline mRNA & Messenger RNA \\
\hline N-terminus & Amino-terminus \\
\hline $\mathrm{NF}-\kappa \mathrm{B}$ & Nuclear factor $\kappa$-light-chain-enhancer of activated B cells \\
\hline NNMT & Nicotinamide N-methyltransferase \\
\hline padj & adjusted p-value \\
\hline PAGE & Polyacrylamide gel electrophoresis \\
\hline PBS & Phosphate-buffered saline \\
\hline PCR & Polymerase chain reaction \\
\hline $\mathrm{PI} 3 \mathrm{~K}$ & Phosphatidylinositol-4,5-biphosphate 3-kinase \\
\hline PPAR & Peroxisome proliferator-activated receptor $\gamma$ \\
\hline PVDF & Polyvinylidene fluoride membrane \\
\hline qPCR & Quantitative polymerase chain reaction \\
\hline Rac1 & RAS-related C 3 botulinum substrate 1 \\
\hline RNA & Ribonucleic acid \\
\hline RPKM & Reads per kilobase of transcript per million mapped reads \\
\hline RPMI & Roswell Park Memorial Institute \\
\hline rRNA & Ribosomal RNA \\
\hline SDS & Sodium dodecyl sulfate \\
\hline SELP & Selectin P \\
\hline shRNA & short hairpin RNA \\
\hline SPSS & Statistical package for the social sciences \\
\hline STAT & Signal transducer and activator of transcription \\
\hline $\mathrm{T}$ & Thymine \\
\hline TAD & Trans-activating domain \\
\hline TBE & Tris-borate-EDTA \\
\hline TBS & Tris-buffered saline \\
\hline TBS-T & Tris-buffered saline-Tween 20 \\
\hline TCA & Tricarboxylic acid \\
\hline TEMED & Tetramethylethylenediamine \\
\hline TGF- $\beta$ & Transforming growth factor- $\beta$ \\
\hline TNF & Tumor necrosis factor \\
\hline
\end{tabular}


Volume diastole

$\mathrm{Vol}_{\mathrm{s}}$

Volume systole

WT

Wild-type 


\section{Acknowledgements}

I sincerely accentuate my endless gratitude and indebtedness to my mentor and supervisor Professor Thomas Meyer. The door to his office was always open whenever I needed help or had a question about my research and writing. He steered me in the right direction whenever I needed it and shaped my experience as a scientist. I could not have imagined having a better advisor and mentor. I feel also extremely grateful to my co-supervisor Dr Alexander Ivetic for his continuous support and the scientific discussions during our meetings. He took an active interest in my work and prompted me to develop my project in a new direction, his lab members Dr. Justin Joachim, Samuel Hart and Yue Yang were all helpful during my 6 months' work in KCL. I am also grateful to my thesis committee members, Professor Dörthe Katschinski, Professor Susanne Lutz, and Dr. Alexander Ivetic for giving me critical feedback on my work and for their valuable suggestions. Thanks are also due to the service unit of the SFB 1002, and their dedicated animal technicians and veterinary surgeons Daniel Kretzschmar, Sarah Zafar, Marcel Zoremba, Beate Knocke, Sabrina Wollborn, and Dr. Verena Reupke for helping me with high scientific and ethical standards to perform LAD ligation surgeries. I would also like to extend my gratitude to the members of the Transcriptome and Genome Analysis Laboratory (TAL), Dr. Gabriela Salinas, Dr. Orr Shomroni and the valuable technical support in RNA-seq experiments from Susanne Luthin and Fabian Ludewig. I am extremely thankful to the International Research Training Group 1816 from the principal investigators, to the students and academic coordination team. I am grateful for the chance I was given for doing my Ph.D. in this program as it provided me with an opportunity to be exposed to infallible guidance of par excellence, professional insights, constructive criticism and generous funding. The research atmosphere was excellent, and it helped to advance my career in diverse directions. I would also like to thank Dr Christina Würtz, Fulya Ören, Ulrike Fischer for their help during my move and stay in Göttingen and London, and all my colleagues in the IRTG 1816 for their delightful company and cooperation. During the past three years, I have received lots of support from our lab members. I find it difficult to succinctly acknowledge Anke Gregus, Heike Hühn, previous Ph.D. student Theresa Riebling and Dr Julia Staab for their continuous support, current Ph.D. student Priyanka Menon, MD and master rotation students, Julian, Till, Jana, Ghazal for their encouragement and after all for their pleasant company. I would like to express my profound gratitude to my neighbor, Saima Zafar and her lovely daughter Arhami who shared very nice food and enjoyable times with me in Göttingen. At the end, I would take this golden opportunity to express my indebtedness to my parents Salah Eldin and Fatima who have been 
my greatest source of inspiration and encouragement at every step in my life. My wholehearted thanks to my lovely sisters and brothers, Aya, Azhar, Anwar, Ahmed and Omar. The time I spent with Ahmed in Germany was full of exciting adventures. I wish him the very best with his future studies. Finally, I would like to express my heartfelt gratitude to my husband, Farhan Cyprian. He has been my constant source of strength and determination and has brought a great deal of pleasure to my life. 


\section{Abstract}

Myocardial infarction (MI), defined as heart damage due to prolonged ischemia, accounts for significant mortality and morbidity and has a substantial financial burden worldwide. While major advancements have been achieved in the characterization of the JAK/STAT signalling pathway in the context of stress and infection, numerous questions on its involvement in cardiac dysfunction and remodeling remain unanswered. In this study, ligation of the left anterior descending coronary artery (LAD) was performed in wild-type and knock-in mice expressing a STAT1 point mutant with defective cooperative DNA binding (STAT1-F77A), to address the role of interferon signalling in acute myocardial infarction. We hypothesized that mice with dysfunctional STAT1 signalling would show a similar phenotype to mice expressing hyperactive STAT3 with respect to its cardioprotective actions. Using echocardiographic evaluation and transcriptomic analysis, we found that a loss of STAT1 tetramerisation protected against adverse cardiac remodeling in female mice. RNA sequencing uncovered numerous immune and metabolic pathways that were differentially regulated in these mice at day 1 post-myocardial injury. The top five immune-related pathways that were upregulated post-MI in both wild-type and F77A mice were cytokinecytokine receptor interactions, chemokine signalling pathways, cell adhesion molecules, regulation of actin cytoskeleton and leukocyte transendothelial migration, whereas the top five metabolism-related pathways downregulated post-MI were oxidative phosphorylation, citrate cycle, fatty acid metabolism, fatty acid degradation, and propanoate metabolism in both wild-type and STAT1-F77A mice. Gene set enrichment analysis revealed a downregulation of cardiac muscle contraction pathway in STAT1-F77A mice only, conceivably due to a heterogeneous cell population of infiltrating immune cells in the infarcted myocardium. In addition, the distinctive transcriptomic profile of infiltrating immune cells in the infarcted area of STAT1-F77A mice was characterized by the upregulation of several immune-activating markers to a higher order of magnitude as compared to their wild-type littermates, including chemokines and their receptors, adhesion molecules, cytokines and their receptors, growth factors and their receptors, coagulation cascade proteins, and heat shock proteins. Notably, the top ten genes differentially expressed in the infarcted area of STAT1-F77A were Fgf23, Cxcl3, Slfn4, Rab44, Cxcl2, Cd177, Gm5483, Illr2, Cd300lf, and Slfn1. The observed upregulation of genes encoding neutrophil markers and adhesion molecules and the significant downregulation of genes engaged in oxidative phosphorylation in the STAT1-F77A mouse line as compared to wild-type mice may act as an adaptive response to reduce oxidative stress, repair cardiac damage and increase 
survival. Additionally, in a parallel plate flow chamber assay, STAT1- and STAT3knockdown HL-60 cells had reduced transendothelial migration when compared to control cells. We conclude that in the early phase of myocardial infarction, a series of transcriptional regulations can probably initiate a beneficial remodeling of the left ventricle in a mouse model expressing dysfunctional STAT1. This study has expanded our understanding of STAT1 transcriptional regulation within the context of murine myocardial infarction. 


\section{Introduction}

Ischemic heart diseases have accounted globally for more than one million deaths in 2017 , making them the first cause of early death. Myocardial infarction (MI) results from acute obstruction of a coronary artery with subsequent myocardial cell death, leading to insufficient pumping of blood to meet the body's demand or what is known as heart failure (Thygesen et al., 2018). Accumulative research in mechanistic and cellular adaptations to cardiac injury has enhanced our understanding of maladaptive cardiac remodeling (Schirone et al., 2017). It is largely accepted that ischemic damage to the cardiac tissue is accompanied by sterile inflammatory responses, where several cytokines play significant roles (Epelman et al., 2015). Until recently, the potential involvement of the JAK/STAT pathway in cardiac repair, regeneration and metabolism has remained largely underestimated. Hence, elucidation of the JAK/STAT signalling pathway in ischemia-induced cardiac dysfunction is relevant for a deeper understanding of the disease outcome. Notably, STAT proteins are known to control a remarkable variety of fundamental biological processes, such as cellular proliferation, differentiation, growth and homeostasis (Abroun et al., 2015). As transcription factors, STAT proteins are characterized by their highly conserved amino acid sequence among different species, including nematodes, insects, and vertebrates (Wang and Levy, 2012). STAT1 and STAT2 were the first two members of STAT protein family to be discovered, followed by five more, namely STAT3, STAT4, STAT5A, STAT5B, and STAT6. Apart from STAT2, all human STAT proteins form homodimers (Kisseleva et al., 2002). Additionally, STATs can be found in heterodimers, e.g. STAT1:STAT2 and STAT1:STAT3, where the magnitude and nature of heterodimeric STATs are influenced by the type of activating ligands (Delgoffe and Vignali, 2013). Since the immunological actions of interferons are not restricted to anti-viral immunity, the investigation of the JAK/STAT pathway is continuously extending our knowledge and generates surprising findings.

STAT1 knock-out mice have reported profound biological defects in response to type I, II, and III interferons and less to other ligands (Akira, 1999). Non-canonical STAT signalling exerts transcriptional regulation in mammals (Majoros et al., 2017), where STAT1 and STAT3 can regulate gene transcription either through binding to DNA or indirectly by several co-activators. Additionally, unphosphorylated STAT3 is known to translocate to the mitochondria and interfere with the energy production by changes in the mitochondrial cellular respiration (Wegrzyn et al., 2009). This extends further connections that link STATs and cellular metabolism, which has newly entered the picture (Dodington et al., 2018). 


\subsection{Discovery of the JAK/STAT signal transduction pathway}

The history of the JAK/STAT signal transduction discovery dates back to the 1980s. At that time, research groups led by James Darnell, Ian Kerr, and George Stark were interested in the question, how gene transcription could be induced within minutes following type I interferons treatment of cells. The rate at which a signal originated at the plasma membrane and transduced to activate target genes in the nucleus, proposed the presence of few intermediate steps. Utilizing different biochemical and genetic techniques, the Darnell, Kerr and Stark laboratories uncovered a previously unrecognized direct signal transduction pathway to the nucleus by identifying the STATs as signal transducers and activators of transcription (Darnell et al., 1994; Fu et al., 1992). Briefly following this pioneering breakthrough, nonreceptor protein tyrosine kinases (pTK) were separately discovered in signalling pathways involving interferons, erythropoietin, and growth hormone receptors (Argetsinger et al., 1993; Velazquez et al., 1992; Witthuhn, et al. 1993). Identical kinases had also come into sight earlier in screening for new pTKs and were called Janus kinases (Firmbach-Kraft et al., 1990; Wilks et al., 1991). Genetic complementation of interferon-unresponsive mutants with recombinant JAK and STAT proteins had made it feasible to reconstitute interferon signalling between the cell membrane and the transcription machinery in the nucleus, with merely two elements: firstly, receptor-associated JAKs that carry the recruitment side for tyrosine phosphorylation of STATs as a first step, and secondly, tyrosine-phosphorylated STATs, which translocate to the nucleus and activate target genes through binding to specific DNA elements (Levy and Darnell, 1990). Currently, the JAK/STAT signal transduction is viewed as a key pathway transducing signals to the nucleus from class I and class II cytokine receptors. Owing to its immense implication in biology, the JAK/STAT pathway is portrayed as a major signalling paradigm (Bousoik et al., 2018). Thus, it is not surprising that JAKs and STATs regulate vital processes in the cell, wherein almost every intricate biological process, from embryogenesis to aging is affected by the JAK/STAT signal transduction (Binari and Perrimon 1994).

\subsubsection{Interferons are categorized as cytokines}

Cytokines are extracellular soluble proteins that convey cell-to-cell signals. They control a plethora of intercellular processes and regulate the recruitment of cells that are involved in inflammation, cellular growth, proliferation, differentiation, apoptosis, angiogenesis, homeostasis, tissue repair, and remodeling. Different families of cytokines bind their cognate receptors on targeted cells, which ultimately determine cellular fate from activation to 
differentiation and survival (Dinarello, 2007). When classified, they can be grouped by their structure and their receptors. One group of cytokines is called interferons (IFNs), which are produced as glycoproteins by a variety of cell types to carry important cell signalling events and trigger various immune responses (Pestka et al., 2007). Notably, interferons can interfere with viral replication, making it less permissive for the virus to replicate inside the cell (Isaacs and Lindenmann, 1957). Classification of interferons is based upon their receptors, to which they bind to in order to carry the signal inside the cell. For example, interferons type I binds to IFNAR (interferon $\alpha / \beta$ receptor) which consists of two chains, IFNAR1 ( $\alpha$ chain) and IFNAR2 ( $\beta$ chain), whereas interferon type II binds to INFGR (interferon- $\gamma$ receptor). There are many type I interferons, namely IFN- $\alpha$, IFN- $\beta$, IFN- $\kappa$, IFN- $\delta$, IFN $-\varepsilon$, IFN- $\tau$, IFN- $\omega$, and IFN- $\zeta$, and only one type II interferon, which is IFN- $\gamma$ (Mesev et al. 2019).

\subsubsection{Structure, function, and regulation of STAT proteins}

The STATs are differentially regulated by a multitude of signalling molecules, including interferons, interleukins, growth factors, and hormones (Ihle et al., 1996). The mammalian STAT family comprises seven STAT proteins, which are STAT1, STAT2, STAT3, STAT4, STAT5a, STAT5b, and STAT6 (Kisseleva et al., 2002). STAT proteins have a size range between 750-900 amino acids. Studying the genomic clustering of STATs and searching for sequence similarity in other eukaryotic species have led to the identification of homologs most closely related to STAT3 and STAT5, in organisms like Dictyostelium (Kawata et al., 1997), C. elegans (Wang and Levy, 2006), and Drosophila (Zeidler et al., 2000). In contrast to Drosophila, where a single STAT transmits the signal through the classical JAK/STAT pathway, the homologs in C. elegans and Dictyostelium signal through different pathways.

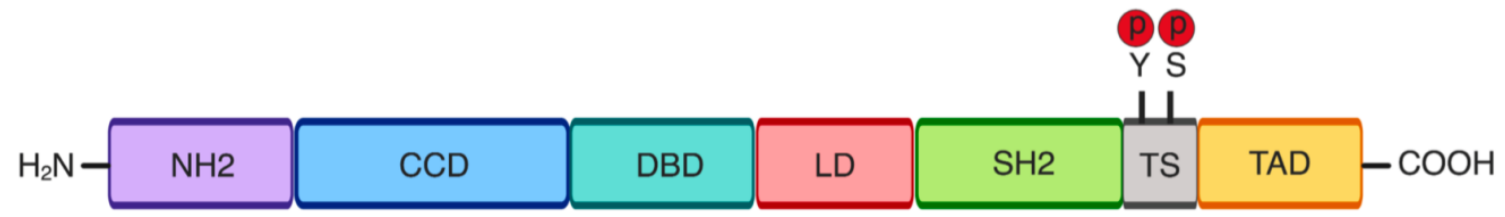

Figure 1: A schematic representation of STAT proteins structure. $\mathrm{NH}_{2}, \mathrm{~N}$-terminus domain; $\mathrm{CCD}$, coiled-coil domain; DBD, DNA-binding domain; LD, linker domain; SH2, Src-homology 2 domain; TS: phosphotyrosyl tail with phosphorylation sites; TAD, transactivation domain.

Utilizing multiple sequence alignment models and analyzing the crystal structure have helped in the identification of six structurally and functionally conserved domains in STAT proteins (Figure 1) (Becker et al., 1998; Chen et al., 1998): (1) The amino terminal domain $\left(\mathrm{NH}_{2}\right)$ being approximately 125 amino acids long, is a well conserved domain that promotes 
homotypic interactions between STAT molecules (Vinkemeier et al., 1998). (2) The coiledcoil domain, located between 135-315 amino acids, is a four-helix bundle that protrudes laterally $(\sim 80 \AA)$ from the core and associates with a number of potentially important regulatory proteins. Hence, it is implicated in nuclear import and export (Begitt et al., 2000). (3) The DNA-binding domain (DBD) (320-480 amino acids), recognizes the palindromic GAS element in activated STAT homodimers (except STAT2), and participates in the process of nuclear import/export (Horvath et al., 1995). (4) The linker domain, localized roughly between 480-575 amino acids, is able to structurally translate the dimerization signal to the DNA-binding motif and regulate basal nuclear export in resting cells (Hüntelmann et al., 2014). (5) The SH2 domain, which is between 575-680 amino acids, is the most highly conserved motif. This domain mediates the specific recruitment to the appropriate receptor as well as the formation of active STAT dimers (Greenlund et al. 1994; Heim et al., 1995). (6) The transcriptional activation domain (TAD) is conserved between mouse and man for every STAT member except STAT2, however, it diverges substantially between STATs. The TAD domain includes conserved serine phosphorylation sites that direct the recruitment of coactivators and in some cases regulate STAT stability (Varinou et al., 2003).

Following phosphorylation of the tyrosine activation motif on the conserved tyrosine residue (701), the phosphotyrosyl residue is subsequently recognized by an SH2 domain on other STAT protomers leading to the formation of active STAT dimers. The amino terminal domain and the SH2 domain are the most conserved structures, they are also involved in proteinprotein interactions, including dimerization, binding to the transcriptional co-activator CREBbinding protein $(\mathrm{CBP} / \mathrm{p} 300)$, and binding to the cytoplasmic receptor (Wojciak et al., 2009; Zhang et al., 1996). The cytoplasmic and nuclear compartment both play an important role in modulating the kinetics of STAT activation. While the activation of the JAK/STAT signal pathway leads to the formation of dimers in parallel conformation by reciprocal phosphotyrosine (pY)-SH2 domain interactions, the antiparallel alignment is present mostly in the absence of stimulation. The parallel conformation positions the two phosphorylated tyrosine residues in opposing SH2-domain pockets, thus protecting STAT1 from dephosphorylation. Therefore, the current notion is that conformational transition from the parallel to the antiparallel alignment renders the phosphotyrosine residue susceptible to enzymatic dephosphorylation and deactivation (Mertens et al., 2006; Wenta et al., 2008; Zhong et al., 2005). Nonetheless, the exact details of these conformational shifts are still a matter of debate. 
Once exported to the cytosol, STATs can enter new cycles of re-activation, nuclear import, inactivation and nuclear export. Apart from canonical tyrosine-phosphorylation, STAT-driven transcription is also regulated by a number of posttranslational modifications, including serine phosphorylation and sumoylation (Droescher et al., 2011; Zhu et al., 1997). Another critical aspect of the JAK-STAT signalling pathway is its short-lived nature, generally confined to less than a few hours. This decline in the cascade is achieved through the activation of opposing regulatory proteins, namely phosphatases and suppressors of cytokine signalling (SOCS) (Adams et al., 1998), along with less well studied regulators such as protein inhibitor of activated STATs (PIAS) (Shuai, 2006), N-myc and STAT interactor (NMI) (Bao and Zervos, 1996), and PDZ and LIM domain-containing protein (SLIM) (Tanaka et al., 2005). Other phosphatases that regulate the JAK-STAT signalling involve: protein tyrosine phosphatase non-receptor type 1 (PTP1B), protein tyrosine phosphatase non-receptor type 2 (TC-PTP), protein tyrosine phosphatase non-receptor type 6 (SHP-1), protein tyrosine phosphatase non-receptor type 11 (SHP-2), and protein tyrosine phosphatase non-receptor Type 13 (PTP-BL). Some of these regulators appear to target receptors and their associated JAKs, while others target the STATs directly and their nuclear export (Aman et al., 1997; Böhmer and Friedrich, 2014).

\subsubsection{STAT1}

STAT1 is a crucial activator of numerous immune effector functions and regulates the expression of genes that are involved in chemokine and cytokine signalling, antigenpresentation, phagocytosis, anti-microbial, and anti-viral immunity. Therefore, expectedly humans carrying variants of STAT1 in the form of nonsense or missense mutations present clinically with a higher predisposition to bacterial and viral infections (Boisson-Dupuis et al., 2012; Casanova et al., 2012). The identification of STAT1 was based on screening of human complementary DNA (cDNA) libraries, that were generated from HeLa cells treated with IFN- $\gamma$ and IFN- $\alpha$ for 45 min and 16 hours, respectively. In particular, molecular cloning and sequence analysis of the cDNA eventually led to the characterization of the two forms p91 and p84, termed as STAT1 $\alpha$ and STAT1 $\beta$, which are the products of alternative splicing of the same gene. Following nuclear import, STAT1 dimers that are not bound to DNA have a conformational change resulting in the exposure of a critical phosphotyrosine residue at position 701 (Staab et al., 2013). This conformational shift renders STAT1 susceptible to the removal of the phosphate group by T-cell protein tyrosine phosphatase (TC-45), which makes the export of STAT1 feasible (ten Hoeve et al., 2002). A compromised dissociation reaction 
of STAT1 from DNA not only hampers the constant search for GAS (gamma-activated sequence) sites, but also impedes with the rate of nucleocytoplasmic shuttling and the transcriptional activity of STAT1 (Yang et al., 2002). Notably, unphosphorylated STAT1 also functions as a transcription factor. However, it is quickly substituted by activated STAT1 dimers following IFN- $\gamma$ stimulation (Yao et al., 2017). Hence, unphosphorylated STAT1 stays for several days in the cell, leading to enhanced signal transduction following re-exposure to low doses of interferon (Cheon and Stark, 2009). Additionally, experimental data suggested the existence of unphosphorylated homodimers, STAT1:STAT2 and STAT1:STAT3 heterodimers before cytokine stimulus (Ho et al., 2016).

\subsubsection{STAT3}

In response to inflammation, binding of acute-phase response factor (APRF) to IL-6responsive elements in the DNA of target genes is known to activate the transcription of acute-phase proteins (APPs) (Gabay et al., 1999; Wegenka et al., 1993). Analysis of APRF revealed a mouse cDNA encoding the 770 amino acid-long homodimers of STAT3. In addition, Northern blot detected a single mRNA transcript $(4.8 \mathrm{~kb})$ in both mouse and human, corresponding to a protein product of $88 \mathrm{kDa}$. Stimulation of mouse liver cells for 3 hours with IL-6 has shown a 5-fold induction of STAT3 mRNA levels (Akira et al., 1994; Zhong et al., 1994). Expression of STAT3 transcripts has been found to be ubiquitous in all tissues examined. Previous research demonstrated that STAT3 can transduce signals from the IL-6 and IL-10 families, as well as granulocyte-colony stimulating factor (G-CSF), leptin, IL-21, IL-27, and potentially IFN-Is (Akira et al., 1997). Moreover, in vitro studies have recently shown that different growth factors and oncogenes are inducing the activation of STAT3, which is associated with the expression of anti-apoptotic/pro-survival genes (Yeh et al., 2009). Deletion of the Stat3 gene in mice resulted in an early lethal embryonic phenotype at embryonic day 6.5-7.5 (Takeda et al., 1997), while tissue specific Stat3 deletions demonstrated an inflammatory phenotype with decreased transformative capacity in cancer cells and tumor regression in animal models (Yu et al., 2009). Moreover, overexpression of the active Stat3 allele, using dominant negative inhibitors and RNA silencing, was associated with increased transformation capacity (Bromberg et al., 1998; Gough et al., 2009; Vultur et al., 2005).

\subsubsection{Type I and II interferon signalling}

The JAK-STAT1 pathway is activated by binding of type I and II interferons to their cognate receptors on the plasma membrane, followed by the activation of non-covalently attached 
Janus-activated kinases (JAKs) bound to these receptors (Darnell et al., 1994; Levy and Darnell, 2002). The mammalian family of JAKs consists of four ubiquitously expressed members: JAK1, JAK2, JAK3, and TYK2, but the expression of JAK3 is primarily limited to the hematopoietic cells. In type I interferon signalling, binding of several ligands induces the dimerization of type I interferon receptor subunits and the phosphorylation of their associated JAKs on specific tyrosine residues. This is followed by the phosphorylation of other tyrosineresidues on the cytoplasmic receptor domains, creating docking sites for latent cytoplasmic STAT molecules, that bind through their Src-homology-2 (SH2) domain. Then, the STATs become phosphorylated by the JAKs on a conserved tyrosine residue near their C-terminus. Upon phosphorylation, the dissociation of STAT molecules from the receptor complex ensues, followed by dimerization via reciprocal phosphotyrosine (pY)-SH2 domain interactions. A complex which comprises STAT1, STAT2, and IRF9, called interferonstimulated gene factor 3 (ISGF3), is subsequently formed. The ISGF3 complex binds DNA on interferon-sensitive response element (ISRE) sequences (5'-AGTTTCN ${ }_{2}$ TTTC-' 3 ), to induce gene transcription of target genes. However, in type II interferon signalling, interferon- $\gamma$ receptor subunits (IFNGR1 and IFNGR2) associate with JAK1 and JAK2, which phosphorylate exclusively STAT1. Two STAT1 monomers form a dimeric complex called gamma-activation factor (GAF), that after translocation to the nucleus and binds GAS sites $\left(5^{\prime}-\mathrm{TTCN}_{3} \mathrm{GAA}^{-} 3\right)$ to activate gene transcription of IFN- $\gamma$-driven target genes. Thus, type I and II interferons translate extracellular signals and mediate differential transcriptional responses (Figure 2) (Lee and Ashkar, 2018). In addition, STATs nuclear translocation is regulated through two pathways, which are the importin $\alpha-5$ dependent pathway and the Ran (RAs-related Nuclear protein) nuclear import pathway (McBride and Reich, 2003). 


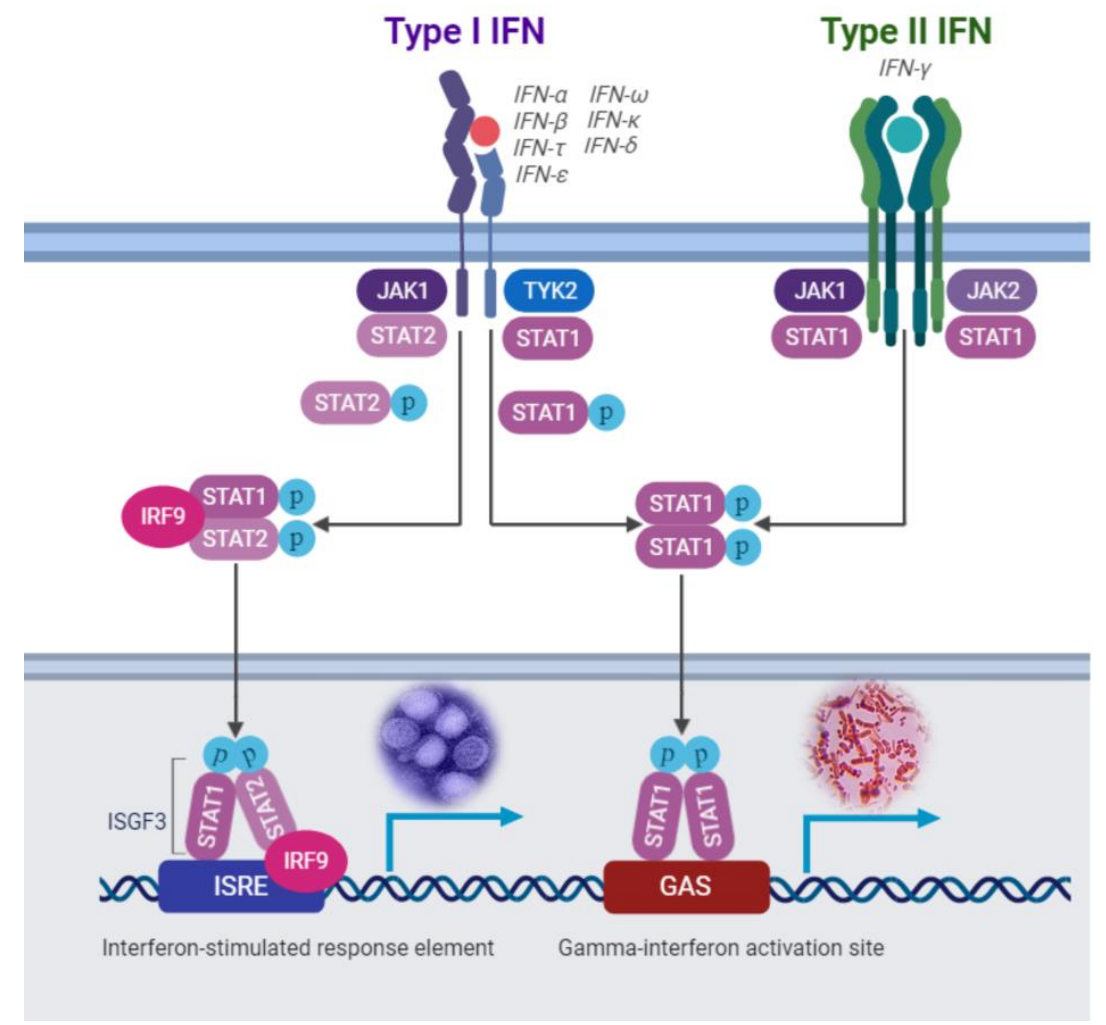

Figure 2: Differential regulation of type I and II interferons signalling pathways. $I F N-\alpha$ (type I IFN) leads to the formation of STAT1-STAT2 heterodimers, which also require IRF9 (interferonregulatory factor 9). This heterotrimeric complex known as ISGF3 (interferon-stimulated gene factor 3 ) drives transcription at so-called ISREs (IFN-stimulated response elements) sites. IFN- $\gamma$ (type II IFN) results in the formation of STATI-STAT1 homodimers that are known as GAF, which binds to other DNA sequence, termed GAS sites, to drive transcription.

\subsubsection{STAT1-cooperative DNA binding}

It has been shown that phage lambda will enter a lytic or a lysogenic cycle by performing a swift switch utilizing cooperative DNA binding (Murray and Gann, 2007). Similarly, STAT dimers polymerize on tandem GAS and ISRE sites through $\mathrm{N}$-terminal domain mediated interactions (Xu et al., 1996). Begitt and colleagues studied the role of cooperative DNA binding in type I and type II interferon signalling. The foundation of their work was the generation of a mutant mouse line (STAT1-F77A), which is defective in polymerizing additional STAT dimers on the DNA, as a result of a single amino acid substitution, namely alanine for phenylalanine at position 77 in the $\mathrm{N}$-terminus domain. Infection experiments using this knock-in mouse line emphasized the physiological significance of STAT1 tetramerisation in immunity. Although STAT1-F77A mice expressed similar levels of STAT1 and responded similarly to vesicular stomatitis virus (VSV) infection, as compared to wildtype littermates, they had a severe defect in antibacterial immunity, exhibiting a higher susceptibility to infection with Listeria monocytogenes. Additionally, STAT1-F77A- 
fibroblasts have shown an impaired induction of IFN- $\gamma$-regulated genes as compared to IFN$\alpha$-regulated genes. Furthermore, using chromatin immunoprecipitation and electrophoretic mobility shift assays, STAT1-F77A recruitment to GAS sites was impeded upon IFN- $\gamma$ stimulation as compared to wild-type cells, but not upon stimulation with IFN- $\alpha$ (Begitt et al., 2014).

\subsubsection{Antagonistic activities of STAT1 and STAT3}

Phosphorylation of STAT proteins by JAKs is essential for facilitating STAT homo- and hetero-dimerization in a parallel dimer conformation. Upon binding of STATs to the receptors, phosphorylation of tyrosine residue 701 in STAT1 and tyrosine residue 705 in STAT3 occurs, followed by STATs dimerization and nuclear translocation to activate responsive genes. Accordingly, tyrosine-phosphorylated STAT1 can form dimers with STAT3 and with STAT2, and the balance between STAT1:STAT3 heterodimers and their STAT3 homodimers will define the transcriptional activity in a cell. Different group of cytokines have been shown to activate distinct STAT proteins. This differential potency of activation for certain cytokines is best exemplified by IFN- $\gamma$, a potent activator of STAT1, and STAT3 mediating signalling from interleukin-6 (IL-6) family members, in particular IL6, leukemia inhibitory factor (LIF) and cardiotrophin-1 (CT-1). Remarkably, STAT1 and STAT3 can substitute for each other absence following IFN- $\gamma$ and IL-6 stimulation, this is characterized by GAS-dependent gene activation in STAT1-null cells, and enhanced activation of STAT1 in STAT3-null cells resulting in IFN- $\gamma$-like response (Costa-Pereira et al., 2002). Every STAT protein has a modular structure comprised of distinct domains. STAT1 and STAT3 display certain homology within the coiled-coiled domain, the DNAbinding domain, the linker domain, the Src-homology (SH2) domain and the carboxy-terminal transactivation domain (TD). To the contrary, the amino-terminal domain is dramatically less conserved between STAT1 and STAT3, signifying this region as a mediator of cellular responses that are unique to distinctive STAT proteins. Even though STAT1 and STAT3 share very comparable conserved structure, both have potential antagonistic effects of their gene products, which regulate processes such as cellular proliferation and apoptosis. For example, several studies have established that STAT1 plays an important role as a tumor suppressor promoting apoptosis in multiple cell types, however, STAT3 exhibits antiapoptotic activities. Additionally, while STAT3 promotes proliferation and transformation in a wide spectrum of carcinomas, STAT1 is reported to have anti-proliferative activities (Table $1)$. 
Table 1: Antagonistic activities of STAT1 and STAT3

\begin{tabular}{cc}
\hline STAT1 & STAT3 \\
\hline anti-proliferative & pro-proliferative \\
pro-apoptotic & pro-survival \\
pro-inflammatory & immunosupressive \\
anti-angiogenic & pro-angiogenic \& pro-metastatic \\
\hline
\end{tabular}

\subsection{The JAK/STAT signalling pathway in cardiovascular diseases}

Several animal models have been developed to investigate the role of various signalling pathways in cardiovascular function under normal or pathologically conditions, including the JAK/STAT signalling pathway, which is a key regulator of several cardiovascular pathologies. Studies have been performed on its function in atherosclerosis, hypertension, myocardial infarction, hypertrophy, myocarditis, and ischemia-reperfusion-induced cardiac injury (El-Adawi et al., 2003; Mascareno et al., 2001; Ortiz-Muñoz et al., 2009; Satou and Gonzalez-Villalobos, 2012; Zhang et al., 2013). In the myocardium, STATs regulate the expression of inflammation- and extracellular matrix-related genes as well as genes regulating apoptosis, angiogenesis and proliferation (Figure 3).

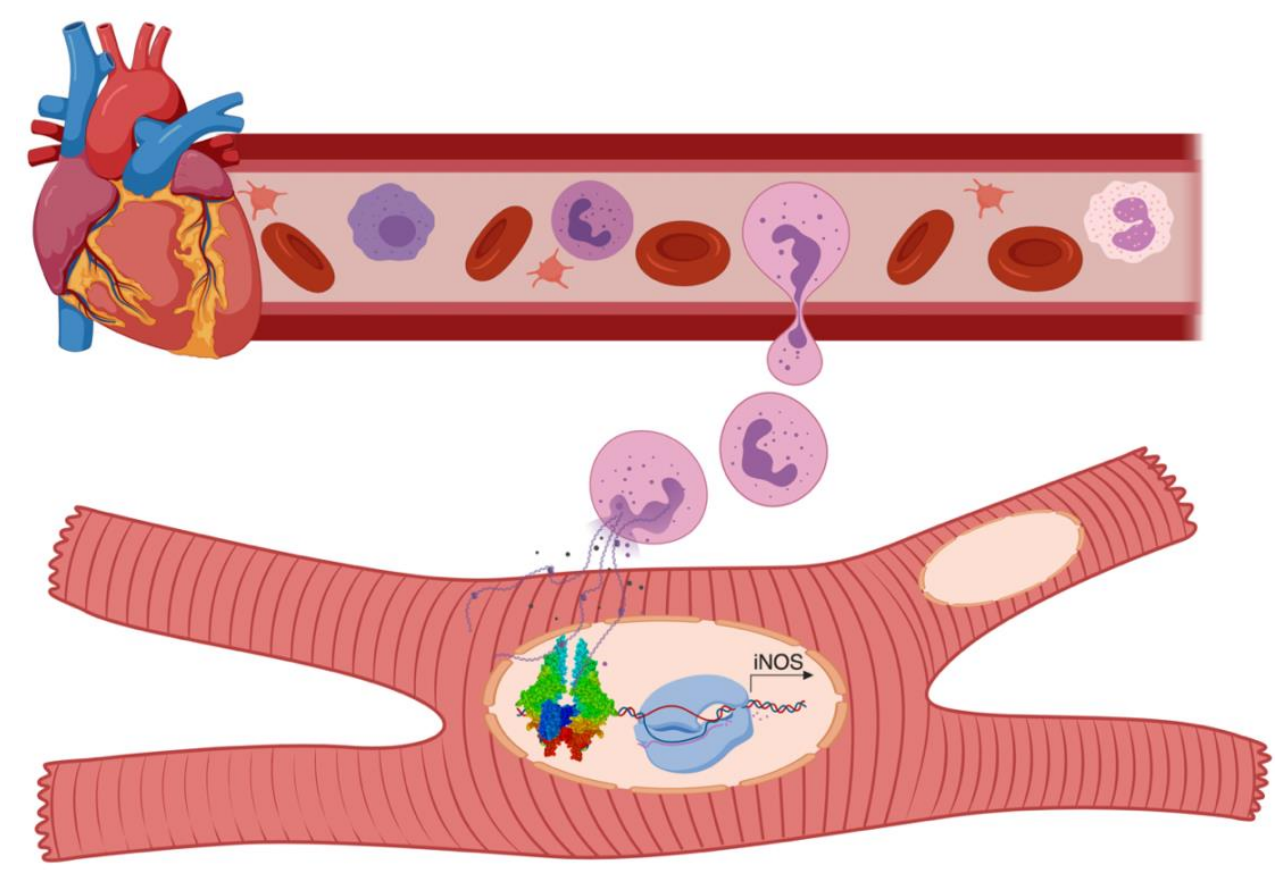

Figure 3: STAT1 and STAT3 regulate transcriptional processes in the ischemic heart. The balance between the activation state of both members of the STAT protein family in the cardiomyocytes as well as in the immune infiltrating cells mediates the remodeling process after myocardial infarction. 


\subsubsection{Myocardial infarction and leukocytes responses}

Myocardial infarction (MI) triggers a cascade of events which recruit different types of immune cells such as neutrophils, macrophages, lymphoid cells, and mast cells to orchestrate several inflammatory responses. Following a cardiac insult, leukocytes move out of the circulation towards the site of injury, guided by a gradient of chemoattractant peptides, known as chemokines (Altara et al., 2016; Frangogiannis, 2014; Gomez et al., 2018; Meng et al., 2016; Nahrendorf and Swirski, 2013; Pinto et al., 2012). Recruited monocytes and neutrophils are initially mobilized from their original niche in the bone marrow to the spleen, generating myeloid cells that take part in multiple repair processes (Lambert et al., 2008; Nahrendorf et al., 2007). Heart-infiltrating immune cells perform a complex role clearing debris and stabilizing the heart wall, through a tuned balance between residents and recruited/differentiated hematopoietic progenitors (Heidt et al., 2014; Massa et al., 2005; Nahrendorf and Swirski, 2016). Hence, trafficking of immune cells shapes the outcome following myocardial infarction by profoundly influencing cardiac repair, fibrosis, regeneration and scar formation, and exerting either pro-inflammatory or anti-inflammatory actions (Forte et al., 2018; Ruparelia et al., 2015). Activated chemokines signal through Gprotein-coupled receptors, which are expressed on various immune cells. Dissociation of the $\alpha$ - and $\beta$ - $\gamma$-subunits of G-proteins leads to downstream signalling cascades which ultimately result in changes in cell polarity and motility through small GTPases (Zweemer et al., 2014). For example, highly coordinated migration and velocity of neutrophils towards sites of injury are established and maintained by cell adhesion molecules (CAMs). Notably, CAMs underpin a crucial cross-talk between innate and adaptive immune cells. They can either anchor the cell to the substratum or transduce signals between adjacent cells to reshape their migratory responses and dynamically remodel the organization of their actin cytoskeleton.

The migration of neutrophils into extravascular tissue can be viewed as a series of interactions which is mediated by (1) the integrin family, (2) the immunoglobulin superfamily, (3) selectins, and (4) cadherins. CAMs can also be classified according to the role they play to (1) recognize antigens, (2) adhere to each other and to the extracellular matrix, and (3) carry costimulation signal (Cavallaro and Dejana, 2011). Additionally, activated chemokine receptors stimulate a rapid elevation of diacylglycerol and cytosolic calcium levels to induce effector functions and coordinate microvascular remodeling, including nitric oxide production and release of reactive oxygen species (ROS) (Cavalera and Frangogiannis, 2014; Saparov et al., 2017). The ensuing removal of dead cells sustains a local environment that supports cardiomyocyte repair. Platelet activation is another hallmark of acute myocardial infarction, 
which can be stimulated by collagen, von Willebrand factor (VWF), thromboxane A2 (TXA2), adenosine diphosphate (ADP), and thrombin. The proceeding thrombus formation within the ventricle increases the myocardium stiffness (Dutta et al., 2012). Furthermore, myocardial ischemia reprograms catabolic and anabolic pathways in the heart to adjust for new requirements of energy acquisition and substrate utilization, and mediate repair processes, cell survival, and growth (Meyer and Voigt, 2017; Wende et al., 2017). Nearly a century ago, Otto Warburg found that cancer cells favor the metabolization of glucose via aerobic glycolysis. Similarly, deregulated metabolism with increased glycolysis has emerged as a significant hallmark of ischemic injury in the heart (Chen et al., 2018). The goal in near future would be to enhance our understanding of the metabolism mediated through the JAK/STAT signalling and propose a rational basis to reprogram metabolic pathways for an improved cardiac repair and regeneration (Doenst et al., 2013).

\subsubsection{Regulation of leucocyte transendothelial migration by the JAK/STAT pathway}

Cell migration is an intricate, synchronized process in which numerous parts of the cell are involved, including surface receptors, intracellular signalling proteins, and the cytoskeleton. Cumulating evidence has highlighted the role of inflammatory cytokines and transcription factors as crucial mediators of cell migration and polarization (Dustin and Chan, 2000; Nieto et al., 1997; Randolph, 2001). One of the best studied examples in cellular polarity is the epithelial-to-mesenchymal transition, in which cells lose epithelial polarity and attachment to adjacent cells (Lamouille et al., 2014). The inverse happens when migrating cells arrive at their target location, build an epithelium and/or integrate into a previous epithelial tissue (Muller, 2015). Examples of such transitions taking place are demonstrated by neural crest development in vertebrates (Bronner and LeDouarin, 2012). Similarly, heart regeneration depends on overlooked temporal and spatial roles for macrophages and neutrophils, where they mediate extracellular matrix regulation. Consequently, improper migration and polarization can potentially affect repair processes and thus contribute to the development of heart failure. STAT1 was found to be essential for IL-6 expression and the increased adhesion and migration of monocytes across the blood-brain barrier, using different in-vitro models in human immunodeficiency virus 1 (HIV-1) infection (Yang et al., 2009). Another study has shown that depletion of STAT1 in a fibroblast cell line resulted in a reduction of cell migration (Xie et al., 2001). Whereas, IFN- $\gamma$ treatment arrested monocyte chemotaxis by modulating the organization of the cytoskeleton via RAC/CDC42 pathways (Hu et al., 2008). Recent studies investigating the loss of STAT3 expression had revealed an elevation of Rac1 
activity in murine embryonic fibroblasts, which mediated a random type of migration through decreasing directional persistence and the assembly of actin stress fibers (Marcella et al., 2005; Teng et al., 2009). These findings suggest a major role of STAT1 and STAT3 in chemokine-induced leucocyte transendothelial migration.

\subsection{Original hypotheses and aims of this thesis}

There is accumulating evidence defining a critical role for cytokines in post-myocardial infarction remodeling. While there is ample evidence indicating a pivotal cardioprotective role of STAT3 in acute and chronic stress in the heart (Zouein et al., 2015), only a small proportion of the studies carried out have investigated the functional role of STAT1. It is proposed that STAT1 deficiency in the heart has a cardioprotective role by enhancing autophagy (McCormick et al., 2012). Accordingly in this thesis, the pleiotropy of STAT1 action and the nature of its transcriptional program in the failing myocardium was studied, with its subsequent impact on cardiac function using genome-wide profiling of the cardiac transcriptome in infarcted areas of wild-type and STAT1 tetramerisation-deficient mice (STAT1-F77A).

This project investigates the biological roles of STAT1-cooperative DNA binding in a murine model of myocardial infarction. In order to test our hypothesis, the following aims were established to:

1) understand the role of STAT1 as a transcription factor and the function of its downstream effector genes responding to myocardial infarction, as well as their potential cross talk in normal and dysfunctional signaling,

2) investigate the potential role of the antiviral program "type-II interferon response" in pathophysiological remodeling, by identifying signalling pathways that are differentially regulated between wild-type and transgenic STAT1-F77A animals following injury of the myocardium, and

3) perform proof-of-concept experiments to study the functional role of STAT1 and STAT3 in mediating transendothelial migration in a neutrophil-like cell line, by generating STAT1- and STAT3-knockdown HL-60 cells. 


\section{Materials and Methods}

\subsection{Materials}

\subsubsection{Chemicals and reagents}

Table 2: List of chemicals and reagents

\begin{tabular}{|c|c|}
\hline Name & Company \\
\hline 3,3’-Diaminobenzidine (DAB) & Sigma-Aldrich, Taufkirchen, Germany \\
\hline 4-(2-Hydroxyethyl)-1- & Carl Roth, Karlsruhe, Germany \\
\hline \multicolumn{2}{|l|}{ piperazineethanesulphonic acid (HEPES, } \\
\hline \multicolumn{2}{|l|}{ Pufferan $\geq 99.5 \%$, p. a.) } \\
\hline Agarose & Carl Roth \\
\hline Ammonium persulphate (APS) & Carl Roth \\
\hline Ampicillin & Sigma-Aldrich \\
\hline Bovine serum albumin (BSA) Fraction V & Carl Roth \\
\hline Bromophenol blue & Fisher Bioreagents, Pittsburgh, USA \\
\hline Chloroform & Sigma-Aldrich \\
\hline Complete mini proteinase inhibitor cocktail & Roche, Rotkreuz, Switzerland \\
\hline Deoxy-adenosine triphosphate (dATP) $\left[{ }^{33} \mathrm{P}\right]-$ & Hartmann Analytic, Braunschweig, \\
\hline labelled & Germany \\
\hline Deoxynucleotide triphosphates (dNTP) & Carl Roth \\
\hline Dimethyl sulphoxide (DMSO) & Applichem, Darmstadt, Germany \\
\hline Dithiothreitol (DTT) & Applichem \\
\hline Entellan & Merck Millipore, Darmstadt, Germany \\
\hline Eosin $Y$ & Merck Millipore \\
\hline Ethanol $(99.8 \%)$ & Carl Roth \\
\hline Ethidium bromide solution $(0.625 \mathrm{~g} / \mathrm{ml})$ & VWR Life Science, Erlangen, Germany \\
\hline Ethylene glycol-bis( $\beta$-aminoethyl ether)- & Carl Roth \\
\hline N,N,N',N'-tetraacetic acid (EGTA) & \\
\hline Ethylenediamine-tetraacetic acid (EDTA) & Sigma-Aldrich \\
\hline Fetal bovine serum (FBS) & Biochrom, Berlin, Germany \\
\hline Fluoromount $\mathrm{G}$ & Southern Biotech, Birmingham, USA \\
\hline Formaldehyde solution (37\%) & Carl Roth \\
\hline L-Glutamine & Thermo Fisher Scientific, Langenselbold \\
\hline
\end{tabular}




\begin{tabular}{|c|c|}
\hline & Germany \\
\hline Glycerol, ROTIPURAN $\geq 99.5 \%$, p. a. & Carl Roth \\
\hline Glycine & Carl Roth \\
\hline HEPES, cell culture grade & Carl Roth \\
\hline Hydrogen peroxide solution (30\%) & Carl Roth \\
\hline IGEPAL CA-630 & Sigma-Aldrich \\
\hline Isopropanol & Carl Roth \\
\hline Kanamycin & Sigma-Aldrich \\
\hline Lithium chloride & Carl Roth \\
\hline Magnesium chloride & Sigma-Aldrich \\
\hline Mayer's haemalum & Merck Millipore \\
\hline Mercaptoethanol & Sigma-Aldrich \\
\hline Methanol & Merck Millipore \\
\hline Methylene blue with (3\%) acetic acid & Stemcell, Cologne, Germany \\
\hline Pefabloc & Roche \\
\hline Penicillin/streptomycin & Biochrom \\
\hline Phenol/chloroform/isoamylalcohol (25:24:1) & Carl Roth \\
\hline Phosphate-buffered saline (PBS) & Life Technologies, Darmstadt, Germany \\
\hline $\begin{array}{l}\text { Polyacrylamide gel electrophoresis running } \\
\text { buffer (SDS-PAGE) }\end{array}$ & Carl Roth \\
\hline $\begin{array}{l}\text { Poly-deoxyinosinic-deoxycytidylic acid (Poly- } \\
\text { dIdC) }\end{array}$ & Sigma-Aldrich \\
\hline Ponceau-S & Sigma-Aldrich \\
\hline Potassium chloride & Merck Millipore \\
\hline Puromycin & Sigma-Aldrich \\
\hline Radioimmunoprecipitation assay buffer (RIPA) & Merck Millipore \\
\hline $\begin{array}{l}\text { Rotiphorese gel } 30 \text { (acrylamid/bisacrylamid } \\
\text { (37.5:1)-solution) }\end{array}$ & Carl Roth \\
\hline $\begin{array}{l}\text { Rotiphorese gel } 40 \text { (acrylamid/bisacrylamid } \\
\text { (29:1)-solution) }\end{array}$ & Carl Roth \\
\hline Roti-plast paraffin & Carl Roth \\
\hline Sodium acetate & Carl Roth \\
\hline Sodium azide & Thermo Fisher Scientific \\
\hline Sodium bicarbonate & Carl Roth \\
\hline
\end{tabular}




\begin{tabular}{ll}
\hline Sodium chloride & Carl Roth \\
\hline Sodium chloride solution $(0.9 \%)$ & B. Braun, Melsungen, Germany \\
\hline Sodium citrate & Carl Roth \\
\hline Sodium deoxycholate & Applichem \\
\hline Sodium dodecyl sulphate (SDS) & Carl Roth \\
\hline Sodium orthovanadate & Acros Organics, Geel, Belgium \\
\hline Sodium pyruvate & Thermo Fisher Scientific \\
\hline Sulfuric acid & Carl Roth \\
\hline Tetramethylethylenediamine (TEMED) & Carl Roth \\
\hline Tris-base & Carl Roth \\
\hline Tris-HCl & Carl Roth \\
\hline Triton X-100 & Carl Roth \\
\hline TRIzol reagent & Thermo Fisher Scientific \\
\hline Tween-20 & Carl Roth \\
\hline Xylene & Merck Millipore \\
\hline
\end{tabular}

Table 3: List of drugs

\begin{tabular}{ll}
\hline Name & Company \\
\hline Cepetor $($ medetomidine, $1 \mathrm{mg} / \mathrm{ml})$ & Cp-pharma, Burgdorf, Germany \\
\hline Fentanyl $(0.05 \mathrm{mg} / \mathrm{ml})$ & Rotexmedica, Trittau, Germany \\
\hline Flumazenil $(0.5 \mathrm{mg} / \mathrm{ml})$ & Inresa Arzneimittel, Freiburg, Germany \\
\hline Isoflurane & Abbvie, Wiesbaden, Germany \\
\hline Ketamine & Medistar, Ascheberg, Germany \\
\hline Midazolam $(5 \mathrm{mg} / \mathrm{ml})$ & Rotexmedica \\
\hline Metamizol $(500 \mathrm{mg} / \mathrm{ml})$ & Zentiva Pharma, Frankfurt am Main, Germany \\
\hline Atipamezole hydrochloride $(5 \mathrm{mg} / \mathrm{ml})$ & Cp-pharma \\
\hline Buprenorphine hydrochloride & Reckitt Benckiser, Slough, UK \\
\hline Xylazine hydrochloride & Ecuphar, Greifswald, Germany
\end{tabular}




\subsubsection{Recombinant proteins and enzymes}

Table 4: List of recombinant proteins and enzymes

\begin{tabular}{ll}
\hline Name & Company \\
\hline IFN- $\alpha$, human, recombinant from $E$. coli & Biomol, Hamburg, Germany \\
\hline IFN- $\gamma$, human, recombinant from $E$. coli & Biomol \\
\hline IL-6, human recombinant & Biomol \\
\hline InnuTaq DNA polymerase & Analytic Jena \\
\hline InnuTaq HOT-A DNA polymerase & Analytic Jena \\
\hline Proteinase K & Carl Roth \\
\hline Taq polymerase & Ares Bioscience, Köln, Germany \\
\hline Trypsin & Sigma-Aldrich \\
\hline Tsp509I & Thermo Fisher Scientific
\end{tabular}

\subsubsection{Water}

Table 5: List of water

Double-distilled $\mathrm{H}_{2} \mathrm{O} \quad$ PURELAB Plus purification system, Elga

Labwater, Celle, Germany

Molecular biology grade water 5 Prime, Heidelberg, Germany

DNase-, RNase- and proteinase-free water

\subsubsection{Kits}

Table 6: List of kits

Name

ABsolute Blue QPCR SYBR Green Mix

dsDNA 905 Reagent Kit

\section{Company}

Thermo Fisher Scientific

Advanced Analytical Technologies,

Heidelberg, Germany

PeqGold total RNA Kit

VWR Life Science

QuantiFluor dsDNA System

Promega

Thermo Scientific Verso cDNA Synthesis Kit

Thermo Fisher Scientific

TruSeq stranded mRNA prep Kit
Illumina, California, USA 


\subsubsection{Culture media}

Table 7: List of media

Name

\section{Company}

Dulbecco's modified Eagle's medium (DMEM) (Glucose

Biochrom

$4.5 \mathrm{~g} / 1,580 \mathrm{mg} / 1 \mathrm{~L}$-glutamine, $110 \mathrm{mg} / 1 \mathrm{Na}$-pyruvate)

DMEM (Glucose $4.5 \mathrm{~g} / \mathrm{l}$, without L-glutamine, $110 \mathrm{mg} / \mathrm{l} \quad$ Biochrom

Na-pyruvate)

RPMI (Roswell Park Memorial Institute medium) $1640 \quad$ Lonza, Cologne, Germany

Endothelial cell growth medium

PromoCell, Heidelberg,

Germany

\subsubsection{Cell lines and animals}

Table 8: List of cell lines

Cell line Characteristics

HL-60 Human suspension cell line derived from peripheral blood acute promyelocytic leukemia, exhibit phagocytic activity and responsiveness to chemotactic stimuli

HUVEC Human umbilical vein endothelial cells are cells derived from the endothelium of veins from the umbilical cord

Table 9: List of animals

\begin{tabular}{lll}
\hline Animal model & Description & \\
\hline STAT1-F77A & Mouse line carrying a point mutation resulting in a & (Begitt et al., \\
knock-in & substitution of alanine in position 77 for phenylalanine & 2014) \\
& on a C57BL6/N background (Charles Rivers, Sulzfeld, \\
& Germany)
\end{tabular}

Mice were kept in individually ventilated cages in the University Medical Centre Animal Facility, Göttingen. Experiments performed were approved by the Lower Saxony State Office for Consumer Protection and Food Safety (Niedersächsisches Landesamt für Verbraucherschutz und Lebensmittelsicherheit, LAVES). 


\subsection{7 shRNA lentiviral transduction particles}

Table 10: List of STAT MISSION shRNA lentiviral transduction particles

Signal transducer and activator of transcription $1,91 \mathrm{kDa}$

SHCLNV-NM_007315

TRCN0000280021

CCGGCTGGAAGATTTACAAGATGAACTCGAGTTCATCTTGTAAATCTTCCAGTTTTTG

TRCN0000280024

CCGGCCCTGAAGTATCTGTATCCAACTCGAGTTGGATACAGATACTTCAGGGTTTTTG

TRCN0000004267

CCGGCTGGAAGATTTACAAGATGAACTCGAGTTCATCTTGTAAATCTTCCAGTTTTT

TRCN0000004265

CCGGCCCTGAAGTATCTGTATCCAACTCGAGTTGGATACAGATACTTCAGGGTTTTT

TRCN0000004266

CCGGCGACAGTATGATGAACACAGTCTCGAGACTGTGTTCATCATACTGTCGTTTTT

Signal transducer and activator of transcription 3 (acute-phase response factor)

SHCLNV-NM_003150

TRCN0000329888

CCGGGGCGTCCAGTTCACTACTAAACTCGAGTTTAGTAGTGAACTGGACGCCTTTTTG

TRCN0000329886

CCGGGCAAAGAATCACATGCCACTTCTCGAGAAGTGGCATGTGATTCTTTGCTTTTTG

TRCN0000329887

CCGGGCACAATCTACGAAGAATCAACTCGAGTTGATTCTTCGTAGATTGTGCTTTTTG

TRCN0000020840

CCGGGCTGACCAACAATCCCAAGAACTCGAGTTCTTGGGATTGTTGGTCAGCTTTTT

TRCN0000020843

CCGGGCAAAGAATCACATGCCACTTCTCGAGAAGTGGCATGTGATTCTTTGCTTTTT

\subsubsection{Primers}

Table 11: List of oligonucleotide primers for genotyping

\begin{tabular}{ll}
\hline Gene & Sequence \\
\hline Stat1-F77A & F: 5'-CGCGAATTGCTAATAAAACAA G-3' \\
& R: 5'-TGCTGCTGAGTCCAAATAAAG-3' \\
Stat1-WT & F: 5'-GGCTGACCTTGGAACTTGTG-3' \\
& R: 5'-AAGAGTCAGCAGGGGTCTGA-3'
\end{tabular}


Table 12: List of oligonucleotide primers for quantitative PCR (qPCR)

\begin{tabular}{|c|c|}
\hline Gene & Sequence \\
\hline \multirow[t]{2}{*}{ Bcl2ll } & F: 5'-GCCTTTTTCTCCTTTGGCGG-3' \\
\hline & R: 5'-TCCACAAAAGTGTCCCAGCC-3' \\
\hline \multirow[t]{2}{*}{$C d 34$} & F: 5'-AGGCTGATGCTGGTGCTAG-3' \\
\hline & R: 5'-AGTCTTTCGGGAATAGCTCTG-3' \\
\hline \multirow[t]{2}{*}{ Cox7a } & F: 5'-AAAACCGTGTGGCAGAGAAG-3' \\
\hline & R: 5'-CAGCGTCATGGTCAGTCTGT-3' \\
\hline \multirow[t]{2}{*}{ Csf3r } & F: 5'-TGAACTACACCCAGGCCTTC-3' \\
\hline & R: 5'-GTGCATGAGGCAGGATAGGT-3' \\
\hline \multirow{2}{*}{ Cxcl2 } & F: 5'-GCAAGGCTAACTGACCTGGA-3' \\
\hline & R: 5'-AGGCACATCAGGTACGATCC-3' \\
\hline \multirow[t]{2}{*}{ Cxcl9 } & F: 5'-GGAGTTCGAGGAACCCTAGT-3' \\
\hline & R: 5'-AGGCAGGTTTGATCTCCGTT-3' \\
\hline \multirow[t]{2}{*}{$G b p 2 b$} & F: 5’-AAGGGCATCTGGATGTGGTG-3' \\
\hline & R: 5'-TCTGGTTGTCACCCTTCTGC-3' \\
\hline \multirow[t]{2}{*}{ Hprt } & F: 5'-AGTCCCAGCGTCGTGATTAG-3' \\
\hline & R: 5'-TGATGGCCTCCCATCTCCTT-3' \\
\hline \multirow[t]{2}{*}{ Irf1 } & F: 5’-ACCCTGGCTAGAGATGCAGA-3' \\
\hline & R: 5'-TGCTTTGTATCGGCCTGTGT-3' \\
\hline \multirow[t]{2}{*}{ Kras } & F: 5'-CAAGAGCGCCTTGACGATACA-3' \\
\hline & R: 5'-CCAAGAGACAGGTTTCTCCATC-3' \\
\hline \multirow[t]{2}{*}{ Nnmt } & F: 5'-GAAGGCAACAGAATGAAGGGAC-3' \\
\hline & R: 5'-TTCCTGAGGGCAGTGCGATA-3' \\
\hline \multirow[t]{2}{*}{$R b 1$} & F: 5'-ACTCCGTTTTCATGCAGAGACTAA-3' \\
\hline & R: 5'-GAGGAATGTGAGGTATTGGTGACA-3' \\
\hline \multirow[t]{2}{*}{ Scal } & F: 5'-AGGAGGCAGCAGTTATTGTGG-3' \\
\hline & R: 5'-CGTTGACCTTAGTACCCAGGA-3' \\
\hline \multirow[t]{2}{*}{ Stat1 } & F: 5'-CCGTTTTCATGACCTCCTGT-3' \\
\hline & R: 5'-TGAATATTCCCCGACTGAGC-3' \\
\hline \multirow[t]{2}{*}{ Thyl } & F: 5'-TCCAGAATCCAAGTCGGAAC-3' \\
\hline & R: 5'-GTTATTCTCATGGCGGCAGT-3' \\
\hline
\end{tabular}

Primers were purchased from Sigma with a grade of purification as desalted. NCBI-BLAST tool was used for primer design. 


\subsubsection{Antibodies}

Table 13: List of primary antibodies

\begin{tabular}{lll}
\hline Name & Company & Cat-No. \\
\hline Ezrin & Cell Signaling Technology, Frankfurt am Main, & 3145 \\
& Germany & \\
\hline Moesin (C-15) & Santa Cruz Biotechnology, Heidelberg, Germany & sc-6410 \\
\hline STAT1(D1K9Y) & Cell Signaling Technology & 14994 \\
\hline pSTAT1 (Tyr701)(58D6) & Cell Signaling Technology & 9167 \\
\hline STAT3 (D1B2J) & Cell Signaling Technology & 30835 \\
\hline STAT3 (H-190) & Santa Cruz Biotechnology & sc-7179 \\
\hline pSTAT3 (Tyr705) (D3A7) & Cell Signaling Technology & 9145 \\
\hline GAPDH (14C10) & Cell Signaling Technology & 2118 \\
\hline$\beta$-actin (8H10D10) & Cell Signaling Technology & 3700
\end{tabular}

Table 14: List of secondary antibodies

\begin{tabular}{llc}
\hline Name & Company & Cat-No. \\
\hline Donkey anti-rabbit secondary antibody & LI-COR Biosciences & $926-32213$ \\
IRDye 800CW IgG $(\mathrm{H}+\mathrm{L})$ & & \\
\hline Donkey anti-mouse secondary antibody & LI-COR Biosciences & $926-32212$ \\
IRDye 800CW IgG $(\mathrm{H}+\mathrm{L})$ & & \\
\hline Donkey anti-Goat secondary antibody & LI-COR Biosciences & $925-68074$ \\
IRDye 680RD IgG $(\mathrm{H}+\mathrm{L})$ & & \\
\hline Biotinylated anti-rabbit IgG $(\mathrm{H}+\mathrm{L})$ & Vector Laboratories, & BA-1000 \\
derived from goat & Peterborough, United Kingdom & \\
\hline
\end{tabular}

\subsubsection{Equipments}

Table 15: List of equipment

\begin{tabular}{ll}
\hline Name & Company \\
\hline 5075 ELV autoclave & Tuttnauer Europe B.V., Breda, Netherlands \\
\hline Bandelin Sonopuls & Bandelin electronic, Berlin, Germany \\
\hline Biological safety cabinet class II & Thermo Fisher Scientific \\
\hline BioPhotometer plus & Eppendorf, Hamburg, Germany
\end{tabular}




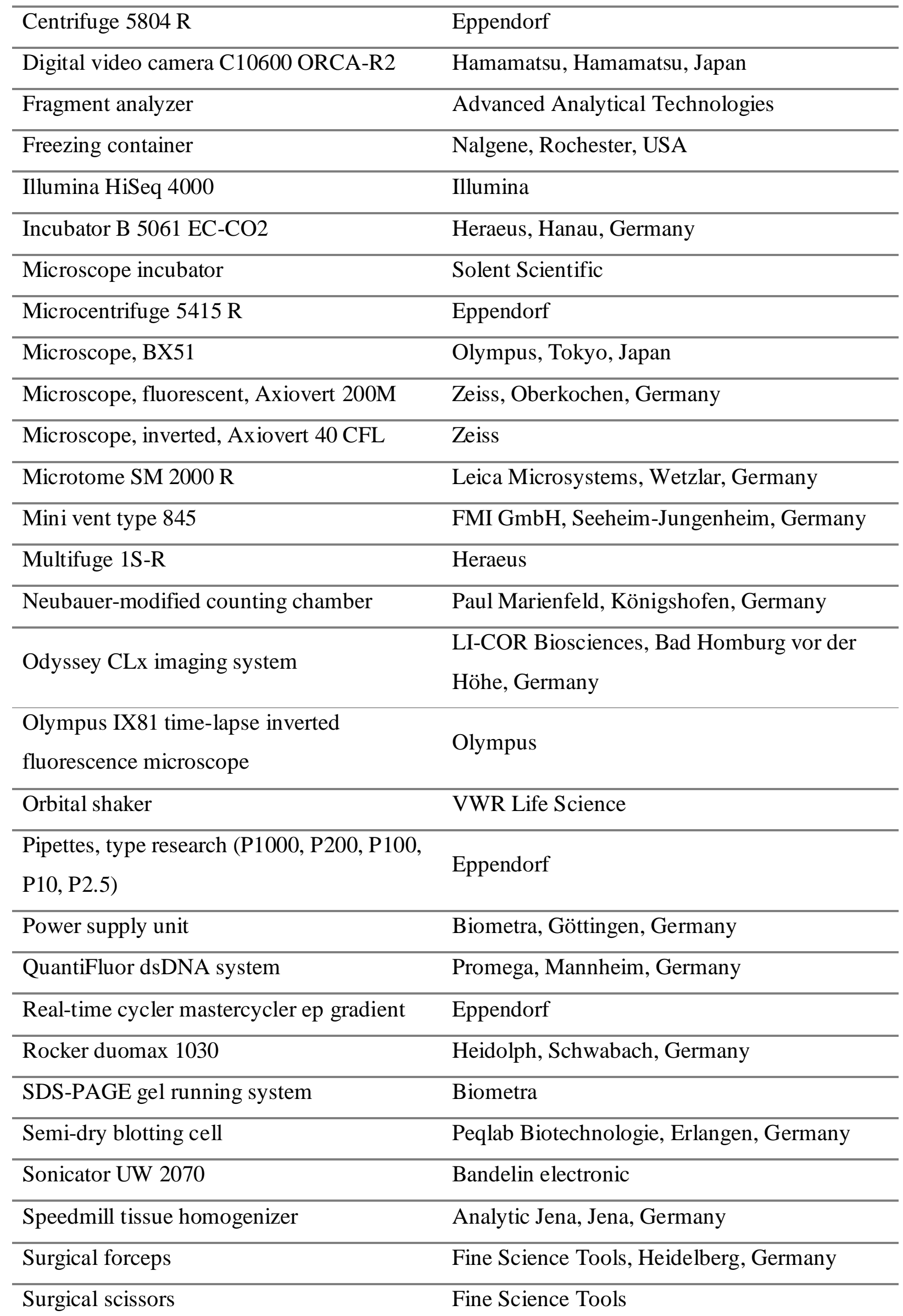




\begin{tabular}{ll}
\hline Thermo shaker TS1 & Biometra \\
\hline Tissue dehydration system TP1028 & Leica Microsystems \\
\hline UVsolo TS imaging system & Analytic Jena \\
\hline Vevo 2100 System & Visualsonics, Toronto, Canada \\
\hline Waterbath GFL & Schuett-biotec, Göttingen, Germany \\
\hline XCell II blot module & Thermo Fisher Scientific \\
\hline XCell SureLock mini-cell electrophoresis & Thermo Fisher Scientific \\
system & \\
\hline
\end{tabular}

\subsubsection{Disposables}

Table 16: List of disposables

\begin{tabular}{ll}
\hline Name & Company \\
\hline Cell culture flasks, T75 & Sarstedt, Nümbrecht, Germany \\
\hline Cell culture plates, 6-well, 24-well, 96-well & Sarstedt \\
\hline Cell scraper $25 \mathrm{~cm}$ & Sarstedt \\
\hline Cryovials & Nunc, Roskilde, Denmark \\
\hline Ethilon 9-0 BV-4 5.0 mm 3/8c EH7448G & Ethicon, Norderstedt, Germany \\
surgical silk & \\
\hline Falcon tubes, 15 ml, 50 ml & Sarstedt \\
\hline Filter (0.22 $\mu \mathrm{m})$ & Carl Roth \\
\hline Glass cover slip & Thermo Fisher Scientific \\
\hline Glass microscope slide & Thermo Fisher Scientific \\
\hline Injection needles & B. Braun, Melsungen, Germany \\
\hline Insulin syringe, 29 G & B. Braun \\
\hline Microreaction tube, 0.2 ml, 1.5 ml, 2 ml & Eppendorf \\
\hline Minisart NY 25 syringe filter & Sartorius, Göttingen, Germany \\
\hline Nitrocellulose membrane & GE Healthcare, Braunschweig, Germany \\
\hline PCR-plate (96 well) & Nunc \\
\hline Petri dishes, 10 cm & Corning, New York, USA \\
\hline Pipette tips (P1000, P100, P10) & Sarstedt \\
\hline Prolene 6-0 C-1 13 mm 3/8c 8889H surgical & Ethicon \\
silk & \\
\hline
\end{tabular}




\begin{tabular}{ll}
\hline $\begin{array}{l}\text { Polyvinylidene fluoride membrane } \\
\text { Immobilon-P (PVDF) }\end{array}$ & Merck Millipore \\
\hline Serological pipettes, $5 \mathrm{ml}, 10 \mathrm{ml}$ & Sarstedt \\
\hline Syringe, $20 \mathrm{ml}, 10 \mathrm{ml}$ & Becton Dickinson, Heidelberg, Germany \\
\hline Tissue culture dish $20 \mathrm{~cm}$ & Nunc \\
\hline Tissue culture plates, 96 well, 24 well, 6 well & Nunc
\end{tabular}

\subsubsection{Software}

Table 17: List of software

\begin{tabular}{|c|c|c|}
\hline Name & Description & Distributor \\
\hline BaseCaller & Base calling for sequence files & Illumina \\
\hline $\begin{array}{l}\text { bcl2fastq } \\
\text { (version } 2.17 .1 .14 \text { ) }\end{array}$ & Formats conversion software & Illumina \\
\hline $\begin{array}{l}\text { biomaRt } \\
\text { (version 2.32.1) }\end{array}$ & $\begin{array}{l}\text { Gene annotation to database } \\
\text { mining }\end{array}$ & Bioconductor $\mathrm{R}$ package \\
\hline BLAST & $\begin{array}{l}\text { Basic local alignment search } \\
\text { tool }\end{array}$ & $\begin{array}{l}\text { National Human Genome Research } \\
\text { Institute, Bethesda, USA }\end{array}$ \\
\hline CorelDraw & Vector graphics editor & Corel, Ottawa, Canada \\
\hline $\begin{array}{l}\text { DESeq2 package } \\
\text { (version 1.14.1.) }\end{array}$ & $\begin{array}{l}\text { Differential gene expression } \\
\text { analysis based on the negative } \\
\text { binomial distribution }\end{array}$ & Bioconductor R package \\
\hline $\begin{array}{l}\text { FastQC } \\
\text { (version } 0.11 .7 \text { ) }\end{array}$ & $\begin{array}{l}\text { Quality control check on } \\
\text { sequences }\end{array}$ & Babraham Institute, Cambridge, UK \\
\hline $\begin{array}{l}\text { featureCounts } \\
\text { (version } 1.4 .5-\mathrm{p} 1 \text { ) }\end{array}$ & $\begin{array}{l}\text { Assigning sequence reads to } \\
\text { genomic features }\end{array}$ & Bioconductor $\mathrm{R}$ package \\
\hline FlowJo & Flow cytometry data analysis & FlowJo LLC, Ashland, USA \\
\hline GraphPad Prism & Analysis and graphing solution & GraphPad, San Diego, USA \\
\hline IBM SPSS & Statistical software, Version 24 & IBM, Kassel, Germany \\
\hline ImageJ & Image processing software & NIH, Bethesda, USA \\
\hline ImageStudio Lite & Image processing software & LI-COR Biosciences \\
\hline Inkscape 2 & Vector graphics editor & Inkscape \\
\hline Realplex ep & $\begin{array}{l}\text { qPCR running and data analysis } \\
\text { software }\end{array}$ & Eppendorf \\
\hline
\end{tabular}




\begin{tabular}{lll}
\hline RStudio & $\begin{array}{l}\text { Open-source integrated } \\
\text { development environment for } \\
\text { statistical computing }\end{array}$ & RStudio Inc. Boston, USA \\
\hline SigmaPlot & $\begin{array}{l}\text { Scientific data analysis and } \\
\text { graphing }\end{array}$ & Systat Software, Erkrath, Germany \\
& DNA cloning and PCR tools & GSL Biotech, Chicago, USA \\
\hline Snapgene & NGS read aligner & National Human Genome Research \\
(version 2.5.2a) & & Institute \\
\hline Vevo 2100 & Ultrasound analysis software & FUJIFILM, Tokyo, Japan \\
& for image data & \\
\hline Volocity Software & $\begin{array}{l}\text { 3D analysis of fluorescence } \\
\text { images }\end{array}$ & PerkinElmer, Baesweiler, USA \\
& $\begin{array}{l}\text { Spreadsheet for calculation and } \\
\text { graphing tools }\end{array}$ & Microsoft, Washington, USA \\
\hline Windows Office & & \\
\hline
\end{tabular}




\subsection{Methods}

\subsubsection{Cell culture}

\subsubsection{Thawing frozen cells}

To unfreeze cells, a cryovial containing $2 \times 10^{6}$ cells was thawed by gentle agitation in a water bath set at $37^{\circ} \mathrm{C}$. When most of the ice started to melt, the vial was removed from the water bath and added immediately to $10 \mathrm{ml}$ of pre-warmed culture medium. The cell suspension was centrifuged at $65 \times \mathrm{g}$ for $6 \mathrm{~min}$ at room temperature. Supernatant was carefully aspirated, leaving the pellet undisturbed to be resuspended in $10 \mathrm{ml}$ of pre-warmed culture medium and cultured in a T75 flask. MHEC5-T cells were cultured in DMEM, supplemented with $10 \%$ FBS and 1\% penicillin/streptomycin. HL-60 cells were cultured in RPMI 1640, supplemented with $10 \%$ FBS and $1 \%$ penicillin/streptomycin, in the additional presence of L-glutamine and $1 \mu \mathrm{g} / \mathrm{ml}$ puromycin.

\subsubsection{Harvesting of cells for cryopreservation}

Cells were harvested by trypsinization after washing with PBS for adherent cells, or by centrifugation for suspension cells. Adherent cells were incubated with trypsin for $2 \mathrm{~min}$, and then centrifuged at $65 \times \mathrm{g}$ for $5 \mathrm{~min}$. Cryopreservation medium was prepared by adding DMSO to FBS at a final concentration of $10 \%(\mathrm{v} / \mathrm{v})$, which was placed on ice until ready to use. The cell pellet was resuspended in $1 \mathrm{ml}$ of cold freezing medium at the recommended viable cell density $\left(1.5 \times 10^{6}-10 \times 10^{6}\right)$. The cryovials were stored at $-80^{\circ} \mathrm{C}$.

\subsubsection{Maintenance of cultured cells}

Cells were maintained at $37^{\circ} \mathrm{C}$ in $5 \% \mathrm{CO}_{2}$ under a humidified atmosphere and passaged every 2 to 3 days. MHEC5-T cells were washed with PBS and subsequently trypsinized for 2 min at $37^{\circ} \mathrm{C}$. Trypsinization was stopped by adding $5 \mathrm{ml}$ of complete culture medium. An aliquot from this suspension was used to seed a new T75 flask with 1:10 dilution. HL-60 cells were counted and then subcultured at a density of $0.5 \times 10^{6}$ cells per ml. Cells were grown at densities no greater than $2.5 \times 10^{6}$ per ml. For protein and RNA analysis, MHEC5-T cells were seeded in a 6-well plate at a density of approximately $1.5 \times 10^{5}$ cells per $\mathrm{cm}^{2}$, and HL-60 cells were seeded in a 6 -well plate at a density of $2 \times 10^{6}$ cells per ml. Absence of mycoplasma infection was routinely checked in the cell lines. 


\subsubsection{Treatment of cultured cells with different stimuli}

MHEC5-T were treated with the respective stimulus (e.g. IFN- $\gamma$, IL-6) after removing the medium and replacing it with cytokine-containing medium at the indicated concentration. For stimulating HL-60 cells, without removing the media, the cytokine was added from an intermediate stock media to achieve the indicated concentration. For RNA extraction, cells were exposed $16 \mathrm{~h}$ prior to stimulation to a medium which had $0.5 \%$ FBS (low serum-culture medium). Stimuli concentrations were the following: human IFN- $\gamma 50 \mathrm{ng} / \mathrm{ml}$, murine IFN $\gamma 5$ $\mathrm{ng} / \mathrm{ml}$, and murine IFN- $\alpha 5 \mathrm{ng} / \mathrm{ml}$, human IL-6 $10 \mathrm{ng} / \mathrm{ml}$, and murine IL-6 $10 \mathrm{ng} / \mathrm{ml}$.

\subsubsection{Lentiviral transduction of HL-60 cells}

Expression of STAT1 and STAT3 genes were knockdown in HL-60 cells using MISSION TRC shRNA lentiviral transduction particles (SIGMA) with pLKO.1 plasmid, using standard puromycin selection method. At the first day, $100 \mu 1$ of HL-60 cells were plated at a density of $1 \times 10^{6}$ cells per $\mathrm{ml}$ in a complete medium in 96-well plates prior to transduction. The lentiviral stock was slowly thawed on ice and then added to each well with a multiplicity of infection (MOI) of 5. At day 4, $100 \mu$ of puromycin was added to the transfected cells with a concentration of $1 \mu \mathrm{g} / \mathrm{ml}$. Cells were observed under the microscope for viability and passaged by adding fresh puromycin-supplemented media according to the cell density. Cells were transferred to a 6-well plate and subsequently cultured in a T25 and then a T75 flask, as they continued to proliferate.

\subsubsection{Protein Analysis}

\subsubsection{Cell lysis and fractionation}

Cells grown on a 6-well plate were washed with PBS, then lysed on ice for 5 min with $50 \mu 1$ of complete cytosolic protein lysis buffer (20 mM HEPES, $10 \mathrm{mM} \mathrm{KCl,} 1 \mathrm{mM}$ EDTA, 100 $\mu \mathrm{M} \mathrm{Na} \mathrm{VO}_{4}, 10 \%$ glycerol, $0.1 \%$ IGEPAL CA-630, supplemented with $1 \%$ Complete proteinase inhibitor cocktail, $3 \mathrm{mM}$ DTT, $0.4 \mathrm{mM}$ Pefabloc, $\mathrm{pH}$ 7.4). Adherent cells were harvested using a cell scraper and then transferred to a $1.5 \mathrm{ml}$ tube. Cells were spun down for $15 \mathrm{sec}$ at $4^{\circ} \mathrm{C}$ and $16100 \times \mathrm{g}$. The supernatant was transferred to a fresh tube and centrifuged again for $5 \mathrm{~min}$ at $4^{\circ} \mathrm{C}$ and $16100 \times \mathrm{g}$. The resulting supernatant was collected as a cytosolic extract. The pellet from the first centrifugation was lysed with $50 \mu$ of complete nucleic protein lysis buffer $\left(20 \mathrm{mM}\right.$ HEPES, $420 \mathrm{mM} \mathrm{KCl,} 1 \mathrm{mM}$ EDTA, $100 \mu \mathrm{M} \mathrm{Na} \mathrm{VO}_{4}, 20 \%$ glycerol, supplemented with $1 \%$ complete proteinase inhibitor cocktail, $3 \mathrm{mM}$ DTT, and 0.4 $\mathrm{mM}$ Pefabloc, $\mathrm{pH}$ 7.4) and incubated for $30 \mathrm{~min}$ on ice, followed by centrifugation for $15 \mathrm{~min}$ 
at $4^{\circ} \mathrm{C}$ and $16100 \times \mathrm{g}$. The supernatant was collected as a nucleic extract. The cytosolic and nucleic extracts were pooled and stored at $-80^{\circ} \mathrm{C}$ for further use. In total, $14 \mu 1$ of $6 \times$ Laemmli buffer $(350 \mathrm{mM}$ Tris-HCl, 8\% SDS, 30\% glycerol, 10\% mercaptoethanol, 0.04\% bromophenol blue, $\mathrm{pH}$ 7.4) were added to each protein extract. Samples were then boiled at $95^{\circ} \mathrm{C}$ for $3 \mathrm{~min}$ and stored at $-20^{\circ} \mathrm{C}$ for Western blotting analysis.

\subsubsection{Protein extraction of HL-60 cells}

HL-60 cells were harvested by centrifugation at $65 \times$ g. Pellets were lysed in $300 \mu 1$ of $2.5 \times$ protein loading buffer (200 mM Tris-HCl pH 6.8, 8\% SDS (v/v), 20\% glycerol (v/v), 0.4\% bromophenol blue (w/v)). Protein extracts were sonicated for few pulses and subsequently incubated at $95^{\circ} \mathrm{C}$ for 5 minutes to complete protein denaturation. Finally, samples were stored at $-20^{\circ} \mathrm{C}$ until further analysis.

\subsubsection{Western blotting analysis}

Protein samples were loaded into the wells of a two-phase 10\% SDS-PAGE gel, along with the molecular weight marker. The stacking gel solution was made by adding $25 \%$ stacking gel buffer (500 mM Tris-HCl, 0.4\% SDS, pH 6.8), 16\% Rotiphorese Gel 30, 0.6\% APS, and $0.2 \%$ TEMED in double-distilled $\mathrm{H}_{2} \mathrm{O}$. The separating gel solution was made by mixing $25 \%$ separating gel buffer (1.5 mM Tris-HCl, 0.2\% SDS, pH 8.8), 33.1\% Rotiphorese Gel 30, $0.03 \%$ APS, and $0.16 \%$ TEMED in double-distilled $\mathrm{H}_{2} \mathrm{O}$. SDS-PAGE running buffer $(25 \mathrm{mM}$ Tris-base, $192 \mathrm{mM}$ glycine, $0.1 \%$ SDS, $\mathrm{pH}$ 8.6) was used to run the gels at $11 \mathrm{~mA}$. A semidry blotting system was used at $80 \mathrm{~mA}$ for $90 \mathrm{~min}$ with transfer buffer ( $25 \mathrm{mM}$ Tris-base, 150 $\mathrm{mM}$ glycine, $10 \%$ methanol, $\mathrm{pH}$ 8.0). A polyvinylidene difluoride (PVDF) membrane was activated with methanol for $1 \mathrm{~min}$ and rinsed with transfer buffer before preparing the stack. The membrane was subsequently blocked for $1 \mathrm{~h}$ at room temperature using blocking buffer, 4\% BSA in Tris-buffered saline (TBS: $137 \mathrm{mM} \mathrm{NaCl}, 10 \mathrm{mM}$ Tris-HCl, pH 7.4) supplemented with $0.1 \%$ Tween-20 (TBS-T). The membrane was incubated with the appropriate dilutions of primary antibody in blocking buffer overnight at $4{ }^{\circ} \mathrm{C}$. The blot was washed five times for 5 min with TBS-T before being incubated with the diluted secondary antibody (prepared in blocking buffer) for $1 \mathrm{~h}$ at room temperature in the dark. The membrane was washed five times with TBS-T and once with TBS, 5 min each. For signal reading, the blot was scanned using the LI-COR Odyssey CLx imaging system. For reblotting, the membrane was washed three times for 15 min in TBS-T, then incubated overnight with a new primary antibody for next day development. When necessary, the blot was stripped with stripping buffer $(62.5 \mathrm{mM}$ Tris- $\mathrm{HCl}, 2 \% \mathrm{SDS}, 0.7 \%$ mercaptoethanol, $\mathrm{pH} 6.8)$ at $60^{\circ} \mathrm{C}$ for $1 \mathrm{~h}$. 
Afterward, it was thoroughly washed with tap water followed by four times TBS-T for 15 min. The blot was blocked with blocking buffer for $1 \mathrm{~h}$ at room temperature and incubated overnight with a new primary antibody. The dilutions of used antibodies were as follows: anti-ezrin 1:1000, anti-GAPDH 1:5000, anti- $\beta$-actin 1:5000, anti-phosphotyrosine-STAT1 1:1000, anti-phosphotyrosine-STAT3 1:1000, anti-STAT1 1:1000, and anti-STAT3 1:1000. The IRDye 800CW-conjugated donkey anti-rabbit secondary antibody used for detection was diluted 1:15000, and IRDye 800CW-conjugated donkey anti-mouse secondary antibody was diluted 1:15000.

\subsubsection{Electrophoretic mobility shift assay}

Native protein samples were incubated with radioactively labelled DNA probes in the presence of the non-specific competitor poly-dIdC. A native polyacrylamide gel was used to run the samples. The reaction mix contained $0.2 \mu \mathrm{l}$ of the DNA probe, which was added to 1 $\mu 1$ poly-dIdC $(2 \mathrm{mg} / \mathrm{ml}), 1.3 \mu \mathrm{l}$ DTT $(100 \mathrm{mM}), 3.5 \mu 1 \mathrm{H}_{2} \mathrm{O}$, and $2.5 \mu 15 \times$ EMSA loading buffer (100 mM HEPES, $200 \mathrm{mM} \mathrm{KCl,} 5 \mathrm{mM} \mathrm{MgCl} 2$, $2.5 \mathrm{mM}$ EDTA, $0.5 \mathrm{mM}$ EGTA, 20\% Ficoll). The reaction mix was incubated for the indicated time points with $4.5 \mu \mathrm{l}$ of protein extracts. In supershift reactions, the STAT1 C-24 antibody was used, while the STAT3 H-190 antibody was used as a negative control. The reactions were loaded onto an equilibrated nondenaturing 4.8\% TBE-polyacrylamide gel (12\% Rotiphorese Gel 40, 2.4\% TBE (89 mM Trisbase, $89 \mathrm{mM}$ boric acid, $2 \mathrm{mM}$ EDTA), $2 \%$ APS, 0.1\% TEMED) and run using $0.25 \times$ TBE at $400 \mathrm{~V}$. After the run, Whatman blotting paper was pressed against the gel and the gel was then vacuum-dried. The radioactive signal was recorded using a phospho-imager film and read by FLA-5100 scanner using TINA v 2.0 software.

\subsubsection{Radioactive labelling of EMSA probes}

Oligonucleotides were diluted at a concentration of $100 \mathrm{mmol} / \mathrm{l}$ in DNase-, RNase- and proteinase-free water and incubated at $95^{\circ} \mathrm{C}$ in a water bath. Mixtures were cooled overnight at room temperature for proper annealing and stored at $-20^{\circ} \mathrm{C}$. Five units of Klenow fragment from E. coli DNA polymerase I were mixed with $5 \mu$ of $10 \times$ Eco-Pol buffer, $0.1 \mathrm{ng}$ annealed oligonucleotides, and $6 \mu \mathrm{l}$ of $\left[{ }^{33} \mathrm{P}\right]$-labelled ATP and incubated for $25 \mathrm{~min}$ at room temperature. Following that, an excess of non-radioactive dNTPs $(6.5 \mathrm{mM}$ of each dNTP) was added for $5 \mathrm{~min}$. The reaction was stopped by the addition of $1 \mu 1$ of $0.5 \mathrm{mM}$ EDTA. Free nucleotides were removed by centrifugation at $700 \times \mathrm{g}$ for $3 \mathrm{~min}$. 


\subsubsection{DNA analysis}

\subsubsection{DNA isolation from mouse tails}

Tail biopsy samples were lysed in $500 \mu \mathrm{l}$ of tail extraction buffer $(100 \mathrm{mM}$ Tris, $5 \mathrm{mM}$ EDTA, $200 \mathrm{mM} \mathrm{NaCl}, 0.2 \% \mathrm{SDS}, \mathrm{pH} 8.5)$ with $10 \mu 1$ proteinase $\mathrm{K}(20 \mathrm{mg} / \mathrm{ml})$ at $56^{\circ} \mathrm{C}$ overnight under continuous shaking at $250 \mathrm{rpm}$. Proteinase $\mathrm{K}$ was inactivated by incubating the samples for $5 \mathrm{~min}$ at $90^{\circ} \mathrm{C}$. Samples were centrifuged at $16100 \times \mathrm{g}$ in $10^{\circ} \mathrm{C}$ for $15 \mathrm{~min}$. $350 \mu \mathrm{l}$ of the resulting supernatant was collected for the DNA precipitation step by adding $500 \mu 1$ of isopropanol. The samples were vortexed and subsequently centrifuged for $5 \mathrm{~min}$ at $16100 \times \mathrm{g}$. The supernatant was removed, and the pellet was washed twice with $70 \%$ ethanol. The air-dried pellets were then resuspended in $50 \mu 1$ of TE buffer $(10 \mathrm{mM}$ Tris-base, $1 \mathrm{mM}$ EDTA, pH 7.5). Heating of DNA samples was performed at $48^{\circ} \mathrm{C}$ for $30 \mathrm{~min}$. The samples were stored at $4^{\circ} \mathrm{C}$ overnight to achieve a complete resuspension in TE buffer.

\subsubsection{Mice genotyping}

Genotyping of STAT1-F77A knock-in mice was performed by combing PCR with a restriction fragment length polymorphism (RFLP) technique. The amplified fragment was generated by mixing the following components in a single reaction mix for each sample: the primer pair stat1-F77A at a final concentration of $0.4 \mu \mathrm{mol} / 1,2.5 \mu 1$ of $10 \times$ reaction buffer, $0.5 \mu \mathrm{l}$ of $50 \mathrm{mM} \mathrm{MgCl} 2$ solution, $1 \mu 1$ of dNTP mix $(10 \mu \mathrm{mol} / 1), 17.25 \mu 1$ of DNase-, RNaseand proteinase-free water, and $0.25 \mu 1$ of Taq polymerase. In total, $1.5 \mu 1$ of purified DNA solution was added to each reaction. The following PCR program was run for the reaction mixtures: $95^{\circ} \mathrm{C}$ for $3 \mathrm{~min}, 41$ cycles of denaturation at $95^{\circ} \mathrm{C}$ for $15 \mathrm{~s}$, annealing at $61^{\circ} \mathrm{C}$ for 15 $\mathrm{s}$, and extension at $72^{\circ} \mathrm{C}$ for $15 \mathrm{sec}$ and a final elongation step at $72^{\circ} \mathrm{C}$ for $7 \mathrm{~min}$. A $1 \%$ agarose gel was run at $85 \mathrm{~mA}$ for 45 min to detect the generated PCR product with the UVsolo TS gel documentation system. Digestion of the fragment was performed by incubating $5 \mu \mathrm{l}$ of the product with $0.3 \mu \mathrm{l}$ of $T$ sp509I, $1.5 \mu \mathrm{l}$ of reaction buffer B and $7.2 \mu 1$ of DNase, RNase- and proteinase-free water at $65^{\circ} \mathrm{C}$ overnight. The digestion reaction mix was analyzed with $3 \%$ agarose gel run at $85 \mathrm{~mA}$ for $60 \mathrm{~min}$. As the F77A mutation causes a loss of Tsp509I site, a 234 bp fragment was detected in homozygous mice, while the wildtypederived PCR product showed two fragments of equal size. 


\subsubsection{RNA analysis}

\subsubsection{RNA extraction from cell culture and reverse transcription}

Cells were cultured in a starvation medium for $16 \mathrm{~h}$ and then stimulated with the respective cytokine for the specified time duration. PeqGold total RNA kit (VWR) was used for RNA isolation. For adherent cells, culture medium was removed and cells were washed with PBS. $400 \mu \mathrm{l}$ of RNA Lysis Buffer T were added directly to the cells on ice. The lysate was transferred directly into a DNA Removing Column placed in a $2.0 \mathrm{ml}$ Collection Tube and centrifuged at $12000 \times \mathrm{g}$ for $1 \mathrm{~min}$ at room temperature. The flow-through lysate was transferred into a new $1.5 \mathrm{ml}$ tube and an equal volume of $70 \%$ ethanol was added to the lysate and mixed thoroughly by vortexing. The lysate was then added directly to the membrane of a PerfectBind RNA column in a new $2.0 \mathrm{ml}$ collection tube. This assembly was centrifuged at $10000 \times \mathrm{g}$ for $1 \mathrm{~min}$. The flow-through liquid and the collection tube were discarded. The column was placed in a fresh $2.0 \mathrm{ml}$ collection tube and washed by adding 500 $\mu \mathrm{l}$ of RNA Wash Buffer I followed by a centrifuge step for $15 \mathrm{sec}$ at $10000 \times \mathrm{g}$. A $600 \mu \mathrm{l}$ of completed RNA Wash Buffer II was added to the column and centrifuged for $15 \mathrm{sec}$ at 10000 $\times \mathrm{g}$. The flow-through liquid was discarded, and the wash step was repeated. The column was placed in the collection tube and centrifuged for $2 \mathrm{~min}$ at $10000 \times \mathrm{g}$ to completely dry the column matrix. To elute RNA, the column was placed into a fresh $1.5 \mathrm{ml}$ microcentrifuge tube and $50 \mu \mathrm{l}$ of sterile RNase-free water were added directly to the binding matrix and centrifuged for $1 \mathrm{~min}$ at $5000 \times \mathrm{g}$. The eluted RNA was used directly for cDNA synthesis or stored at $-80^{\circ} \mathrm{C}$. Verso cDNA Synthesis kit was used to reverse transcribe the RNA to create cDNA, according to the manufacturer's instructions. The reaction mixture was as follows: 4 $\mu 1$ of $5 \times$ cDNA synthesis buffer, $2 \mu l$ of dNTP mix, $1 \mu 1$ of RNA primer (anchored Oligo dT), $1 \mu \mathrm{l}$ of RT Enhancer, $1 \mu \mathrm{l}$ of Verso Enzyme Mix, and $3 \mu \mathrm{l}$ of DNase-, RNase- and proteinasefree $\mathrm{H}_{2} \mathrm{O}$ with $8 \mu$ l of the purified RNA. The amount of necessary RNA had been determined experimentally. The reverse transcription cycling program was $30 \mathrm{~min}$ at $42^{\circ} \mathrm{C}$, followed by inactivation cycle at $95^{\circ} \mathrm{C}$ for $2 \mathrm{~min}$. The samples were stored at $-20^{\circ} \mathrm{C}$.

\subsubsection{Real-time PCR}

Real-time reverse transcription PCR was used to enable detection and measurement of gene transcription products. The PCR cycling program was as follows: $95^{\circ} \mathrm{C}$ for $4 \mathrm{~min}, 38$ cycles of denaturation at $95^{\circ} \mathrm{C}$ for $45 \mathrm{sec}$, annealing at $61^{\circ} \mathrm{C}$ for $45 \mathrm{sec}$, and extension at $72^{\circ} \mathrm{C}$ for $120 \mathrm{sec}$, with a final elongation at $72^{\circ} \mathrm{C}$ for $10 \mathrm{~min}$. A master mix was prepared for each reaction containing $10 \mu \mathrm{l}$ of SYBR green, $0.28 \mu \mathrm{l}$ of each primer $(5 \mu \mathrm{mol} / 1)$, and $9.44 \mu \mathrm{l}$ of 
$\mathrm{H}_{2} \mathrm{O}$. Using a semi-skirted 96-well-plate, $19 \mu \mathrm{l}$ of the master mix was distributed to each well with $1 \mu \mathrm{l}$ of the cDNA reaction sample. GAPDH or HPRT were used as a reference housekeeping gene and each sample was run in duplicate. The $2^{-\Delta \Delta \mathrm{CT}}$ method was used to analyze the data exported from the realplex ep program.

\subsubsection{Animal experiments}

\subsubsection{Left anterior descending coronary artery ligation in mice}

STAT1-F77A knock-in mice were used in the myocardial infarction model (LAVES approved with reference number 3392 42502-04-13/1226). Surgery was performed on healthy young female animals at the age of 8-12 weeks. To provide basic analgesia, metamizol in a final concentration of $2 \mathrm{mg} / \mathrm{ml}$ was added to drinking water three days before surgery. Mice were intraperitoneally anaesthetized with $10 \mu \mathrm{l} / \mathrm{g}$ body weight of 5\% Cepetor, $10 \%$ midazolam, and $10 \%$ fentanyl in $0.9 \% \mathrm{NaCl}$ solution and stabilized in the supine position. A small incision of the trachea was introduced to insert a tube for artificial respiration $(150 \mu 1$ volume and 150 strokes/min). To expose the heart, a lateral cut of the pectoral muscles was carried to expose the rib cage and spread the fourth intercostal space. Using Ethilon 9-0 BV-4 $5.0 \mathrm{~mm} \mathrm{3/8c}$ EH7448G surgical silk, ligation of the left anterior descending artery was performed to induce infarction. Cardiac apex started discoloration into white as a measure of proper occlusion of the coronary vessel. Sham-operated mice underwent the same procedure except occlusion of the left anterior descending artery. Following successful ligation, retractors were disconnected, and suturing of the skin was performed using Prolene 6-0 C-1 $13 \mathrm{~mm} \mathrm{3/8c}$ $8889 \mathrm{H}$ surgical silk. Intraperitoneal injection of $10 \mu \mathrm{l} / \mathrm{g}$ body weight of narcosis antagonist, $5 \%$ atipamezole, $10 \%$ flumazenil, in sterile $0.9 \% \mathrm{NaCl}$ was administered. Mice were given $1 \%$ buprenorphin in sterile $0.9 \% \mathrm{NaCl}$ at a dose of $15 \mu \mathrm{l} / \mathrm{g}$ body weight and placed on a prewarmed plate at $37^{\circ} \mathrm{C}$ until awake. Mice were sacrificed through $\mathrm{CO}_{2}$ asphyxiation and hearts were collected at day 1 following the surgery. The whole heart was washed with $1 \times$ PBS and the infarcted areas from the left ventricle were excised with a surgical blade. Samples were collected in TRIzol and stored at $-80^{\circ} \mathrm{C}$ until further analysis.

\subsubsection{Immunohistochemistry of paraffin-embedded sections}

Mice were sacrificed through $\mathrm{CO}_{2}$ asphyxiation. In LAD ligation experiments, the heart was surgically removed and washed in PBS before being stored in $4 \%$ formaldehyde solution at $4^{\circ} \mathrm{C}$. After 24 hours, organs were transferred from paraformaldehyde (PFA) into PBS and stored at $4{ }^{\circ} \mathrm{C}$ until further processing. For paraffin embedding, organs were prepared through 
dehydration using ascending concentrations of ethanol and finally pure xylene. Casting paraffin-embedded organs in blocks was performed using the EG1160 tissue embedder. The blocks were thren cut into sections at $3 \mu \mathrm{m}$ thickness using the SM $2000 \mathrm{R}$ microtome. Tissues were left to stretch in a water bath at $45^{\circ} \mathrm{C}$ before transferring them into a slide to dry at $50^{\circ} \mathrm{C}$. To monitor protein expression and localization of proteins in tissues, deparaffinization and rehydration were performed by xylene incubation for $15 \mathrm{~min}$ twice, then by using descending concentrations of ethanol $\left(99 \%, 96 \%, 70 \%\right.$, desalted $\left.\mathrm{H}_{2} \mathrm{O}\right)$ twice for 2 min per each solution. Citrate buffer ( $8.13 \mathrm{mM}$ sodium citrate, $\mathrm{pH}$ 6.0) was used for boiling the samples for $15 \mathrm{~min}$ in a microwave. Samples were cooled on ice followed by washing with double-distilled $\mathrm{H}_{2} \mathrm{O}$ followed by PBS. A solution of $3 \% \mathrm{H}_{2} \mathrm{O}_{2}$ in PBS was used for 20 min at $4^{\circ} \mathrm{C}$ to block peroxidase activity in the tissue sections. A blocking solution consisting of $10 \%$ FBS in PBS, supplemented with $0.05 \%$ Triton X-100, was used to incubate the slides for $1 \mathrm{~h}$ at room temperature. Primary antibodies were incubated in 10\% FBS in PBS overnight at $4^{\circ} \mathrm{C}$. Tissue slides were washed three times in PBS before secondary antibody incubation, which was diluted in 10\% FBS in PBS at 1:1000 for $1 \mathrm{~h}$ at room temperature. Tissue slides were washed four times with PBS followed by incubation for $1 \mathrm{~h}$ with streptavidin/peroxidase solution diluted at 1:1000 in PBS. A 3,3'-diaminobenzidine (DAP) staining was used at development stage (2.5\% stock was used by diluting it 1:250 in PBS and adding $0.01 \%$ $\mathrm{H}_{2} \mathrm{O}_{2}$ ). Reaction time was between 5 to $10 \mathrm{~min}$ and was stopped by rinsing with doubledistilled water. Slides were incubated in filtered Mayer's haemalum solution for $10 \mathrm{~min}$ followed by washing in running tap water for $10 \mathrm{~min}$. Slides were then washed twice with desalted water and dehydrated with ascending concentrations of ethanol (70\%, 96\%, 99\%) twice for 2 min each. Tissues were incubated in xylene for 15 min twice. Entellan was used to mount the samples to be examined using a light microscope. Dilutions of primary antibodies were as the following: anti-STAT1; 1:200, anti-STAT3; 1:200. 
Table 18: Evaluation of STAT staining distribution and intensity in heart tissue

\begin{tabular}{ll}
\hline 0 & No specific staining \\
\hline 1 & Less than half of the left ventricle \\
\hline 2 & $50 \%$ to $90 \%$ of the left ventricle \\
\hline 3 & Entire left ventricle but little or no infiltration of the septum or right ventricle \\
\hline 4 & $\begin{array}{l}\text { Entire left ventricle and moderate to significant infiltration of the right } \\
\text { ventricle or the septum }\end{array}$ \\
\hline 0 & No specific staining \\
\hline 1 & Single cells at low density \\
\hline 3 & Small accumulations, moderate density \\
\hline 4 & Many or large accumulations \\
\hline
\end{tabular}

\subsubsection{RNA sequencing and analysis}

Sequencing of RNA samples was conducted at the Microarray and Deep-Sequencing Facility (Transcriptome and Genome Analysis Laboratory (TAL), Göttingen). RNA was extracted using TRIzol isolation protocol. Quality and integrity of RNA samples were assessed with a fragment analyzer using the standard sensitivity RNA analysis kit. All samples selected for sequencing exhibited an RNA integrity number over 8 . For library preparation, $500 \mathrm{ng}$ of total RNA was used. Samples were immediately fragmented (300 bp) and cDNA subsequently synthesized. RNA-seq libraries were performed using TruSeq stranded mRNA prep kit with a modified strand-specific massively-parallel cDNA sequencing mRNA-seq protocol. The protocol was first optimized during the ligation step by diluting the adapters concentration to increase ligation efficiency (>94\%), and finally reducing the number of PCR cycles to avoid PCR duplication artifacts as well as primer dimers in the final library product. Using strandspecificity protocols, differences in coverage, agreement with known annotations, and accuracy for expression profiling were observed. For accurate quantitation of cDNA libraries, a fluorometric-based system called QuantiFluor dsDNA was used. The size of final cDNA libraries was determined by using the dsDNA 905 reagent kit, exhibiting a sizing of $300 \mathrm{bp}$ in average. Libraries were pooled and sequenced on an Illumina HiSeq 4000 generating 50 bp single-end reads (30-40 million reads/sample). Generation of raw data files and their quality check were performed, and sequence images were transformed with BaseCaller to bcl files, 
which was demultiplexed to FASTQ files with bcl2fastq. The sequencing quality was asserted using FastQC. Mapping of sequenced reads and read counting for all samples was performed. Sequences were aligned to the reference genome Mus musculus mm10 (version 89) using the STAR aligner, allowing for 2 mismatches within 50 bases (Dobin et al., 2013). Subsequently, read counting was performed using featureCounts (Liao et al., 2014). Differential expression analysis was also performed and read counts were analyzed in the R/Bioconductor environment (version 3.4.2) using DESeq2 package (Love et al., 2014). Candidate genes were filtered using an absolute $\log _{2}$ fold-change $>1$ and FDR(False Discovery Rate)-corrected $p$ value $<0.05$. Gene annotation was performed using Mus musculus entries via biomaRt $\mathrm{R}$ package (Durinck et al., 2009). The following R packages were used for gene set enrichment analysis and data visualization: ggplot2, kableExtra, pheatmap, d3heatmap, gage, dplyr, pathview, biomaRt, grid, VennDiagram, and ggrepel.

\subsubsection{Parallel plate flow chamber assay}

All cells were resuspended in HL-60 media (RPMI-1640, 10\% FCS, 1\% penicillin/ streptomycin) supplemented with $25 \mathrm{mM}$ HEPES buffer prior to the perfusion assay. Addition of HEPES provided supplemental buffering at a $\mathrm{pH} 7.2$ through 7.6, as $\mathrm{CO}_{2}$ supply was absent in the assay. A monolayer of human umbilical vein endothelial cell (HUVEC) was grown to confluency and stimulated overnight with $10 \mathrm{ng} / \mathrm{ml}$ TNF- $\alpha$. On the next day, analysis of HL-60 cells interacting with HUVEC was performed at $37^{\circ} \mathrm{C}$ in a $5 \% \mathrm{CO}_{2}$ incubator and visualized using Olympus IX81 time-lapse inverted fluorescence microscope linked to a Hamamatsu C10600 ORCA-R2 digital video camera. A Harvard syringe pump generated a physiological shear stress of blood flow with a flow rate of $0.30 \mathrm{ml} / \mathrm{min}$ which corresponds to $1.5 \mathrm{dyn} / \mathrm{cm}^{3}$. HL-60 cells were perfused at a density of $1 \times 10^{6}$ cells $/ \mathrm{ml}$ over TNF- $\alpha$ activated HUVEC, grown in plastic dishes for 25 minutes. The footage was recorded from the moment the cells were visible using a 4 ×objective. Images were saved into a video file using Volocity Imaging software.

\subsubsection{Statistical analysis}

IBM SPSS and R statistical software were used. Descriptive statistics were calculated from the data (e.g. mean, confidence interval of the mean, median, minimum, maximum, first quantile, third quantile, standard deviation, and standard error). A t-test for independent samples was used to compare the means for two groups. A p-value of 0.05 was chosen as level of significance. 


\section{Results}

\subsection{Echocardiographic analysis demonstrated beneficial outcomes in STAT1-F77A mice after myocardial infarction}

The left ventricular size and function of STAT1-F77A and wild-type mice were assessed using sham-operated mice (as control) or mice that were subjected to ligation of the left anterior descending coronary artery (LAD), as published in Theresa Riebling's doctoral thesis, summarized in Tables 21 and 22 and depicted in Figure 4. The left ventricular endsystolic inner diameter in STAT1-F77A mice was significantly decreased as compared to wild-type mice on the third day (mean $=3.92 \mathrm{~mm}, 95 \%-\mathrm{CI}=3.59-4.25 \mathrm{~mm}$ vs mean $=4.39 \mathrm{~mm}$, $95 \%-\mathrm{CI}=4.15-4.64 \mathrm{~mm}, \mathrm{p}=0.036$ ) and at the end of the first week (mean=4.38 mm, 95\%$\mathrm{CI}=3.70-5.05 \mathrm{~mm}$ vs mean $=5.40 \mathrm{~mm}, 95 \%-\mathrm{CI}=4.60-6.20 \mathrm{~mm}, \mathrm{p}=0.049)$ after LAD ligation. Similarly, the left ventricular end-systolic area in STAT1-F77A mice was significantly lower as compared to wild-type mice on the third day (mean $=10.24 \mathrm{~mm}^{2}, 95 \%-\mathrm{CI}=8.92-11.55 \mathrm{~mm}^{2}$ vs mean $\left.=12.79 \mathrm{~mm}^{2}, 95 \%-\mathrm{CI}=11.54-14.05 \mathrm{~mm}^{2}, \mathrm{p}=0.0068\right)$ and at the end of the first week (mean $=11.55 \mathrm{~mm}^{2}, 95 \%-\mathrm{CI}=7.79-15.31 \mathrm{~mm}^{2}$ vs mean $=18.09 \mathrm{~mm}^{2}, 95 \%-\mathrm{CI}=13.54-22.65$ $\mathrm{mm}^{2}, \mathrm{p}=0.03$ ) after LAD ligation. The systolic epicardial area was reduced at day 7 post-MI compared to wild-type mice (mean=22.6 $\mathrm{mm}^{2}, 95 \%-\mathrm{CI}=18.36-26.84 \mathrm{~mm}^{2}$ vs mean=29.52 $\left.\mathrm{mm}^{2}, 95 \%-\mathrm{CI}=24.32-34.71 \mathrm{~mm}^{2}, \mathrm{p}=0.039\right)$. Additionally, the left ventricular end-diastolic inner diameter in STAT1-F77A mice was significantly decreased on the third day compared to wild-type mice (mean=4.39 $\mathrm{mm}, 95 \%-\mathrm{CI}=4.14-4.64 \mathrm{~mm}$ vs mean=4.79 $\mathrm{mm}$, 95\%$\mathrm{CI}=4.64-4.94 \mathrm{~mm}, \mathrm{p}=0.0035)$. Correspondingly, the end-diastolic area in STAT1-F77A mice was significantly lower on the third day compared to wild-type mice (mean=13.57 $\mathrm{mm}^{2}$, $95 \%-\mathrm{CI}=12.17-14.98 \mathrm{~mm}^{2}$ vs mean $\left.=15.83 \mathrm{~mm}^{2}, 95 \%-\mathrm{CI}=14.81-16.85 \mathrm{~mm}^{2}, \mathrm{p}=0.013\right)$. 
Table 19 illustrates the most common parameters used to evaluate the function of the left ventricle including the equations used to calculate them, which are fractional area shortening (FAS), fractional shortening (FS), end-systolic volume ( $\left.\mathrm{Vol}_{\mathrm{s}}\right)$, end-diastolic volume $\left(\mathrm{Vol}_{\mathrm{d}}\right)$, and ejection fraction (EF). In our data, the fractional area shortening of the left ventricle in STAT1-F77A mice was significantly increased at the end of the first week following myocardial infarction as compared to wild-type mice (mean $=26.92 \%, 95 \%$-CI $=20.36-33.48 \%$ vs mean $=16.13 \%, 95 \%-\mathrm{CI}=9.70-22.56 \%, \mathrm{p}=0.024)$. Likewise, the ejection fraction of the left ventricle in STAT1-F77A mice was significantly improved at day 7 following MI as compared to wild-type mice $($ mean $=30.11 \%, 95 \%-\mathrm{CI}=22.64-37.57 \%$ vs mean $=18.6 \%, 95 \%$ $\mathrm{CI}=11.26-25.95 \%, \mathrm{p}=0.03$ ). Furthermore, the end-systolic volume of the left ventricle in STAT1-F77A mice was significantly decreased as compared to wild-type mice on the third day $\left(\right.$ mean $=62.44 \mathrm{~mm}^{3}, 95 \%-\mathrm{CI}=52.42-72.45 \mathrm{~mm}^{3}$ vs mean=79.49 $\mathrm{mm}^{3}, 95 \%-\mathrm{CI}=69.93-$ $\left.89.05 \mathrm{~mm}^{3}, \mathrm{p}=0.039\right)$ and at the end of the first week (mean $=72.00 \mathrm{~mm}^{3}, 95 \%-\mathrm{CI}=42.88-$ $101.12 \mathrm{~mm}^{3}$ vs mean $\left.=119.69 \mathrm{~mm}^{3}, 95 \%-\mathrm{CI}=84.14-155.23 \mathrm{~mm}^{3}, \mathrm{p}=0.039\right)$. Moreover, the end-diastolic volume of the left ventricle in STAT1-F77A mice was significantly lower on the third day as compared to wild-type mice (mean $=85.82 \mathrm{~mm}^{3}, 95 \%-\mathrm{CI}=74.52-97.12 \mathrm{~mm}^{3} \mathrm{vs}$ mean $\left.=101.90 \mathrm{~mm}^{3}, 95 \%-\mathrm{CI}=93.03-110.78 \mathrm{~mm}^{3}, \mathrm{p}=0.045\right)$.

Table 19: Equations used in the calculation of left ventricular function parameters

\begin{tabular}{|ll|}
\hline FAS & $\left(\right.$ Area $_{\mathrm{d}}-$ Area $\left._{\mathrm{s}}\right) /$ Area $_{\mathrm{d}} * 100$ \\
\hline $\mathrm{FS}$ & $\left(\mathrm{LVID}_{\mathrm{d}}-\mathrm{LVID}_{\mathrm{s}}\right) / \mathrm{LVID}_{\mathrm{d}} * 100$ \\
\hline $\mathrm{Vol}_{\mathrm{s}}$ & $(5 / 6) *\left(\right.$ Area $\left._{\mathrm{s}} * \mathrm{~L}_{\mathrm{s}}\right)$ \\
\hline $\mathrm{Vol}_{\mathrm{d}}$ & $(5 / 6) *\left(\right.$ Area $\left._{\mathrm{d}} * \mathrm{~L}_{\mathrm{d}}\right)$ \\
\hline $\mathrm{EF}$ & $\left(\mathrm{Vol}_{\mathrm{d}}-\mathrm{Vol}_{\mathrm{s}}\right) / \mathrm{Vol}_{\mathrm{d}} * 100$ \\
\hline
\end{tabular}


A
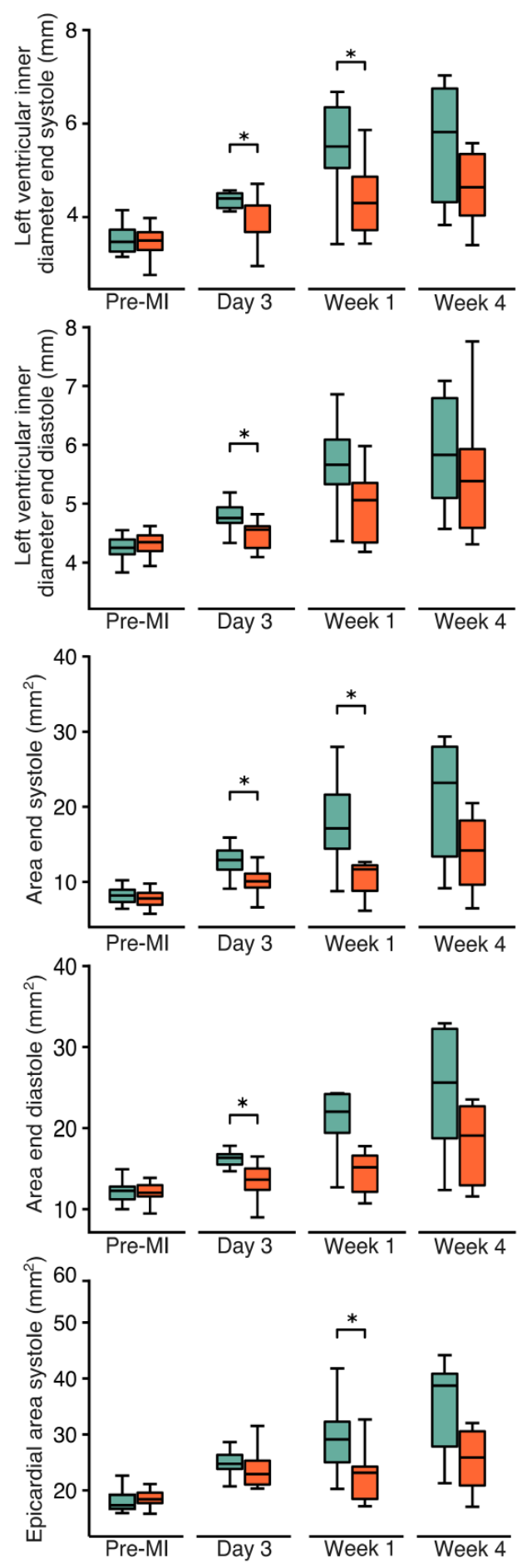

$\mathrm{B}$
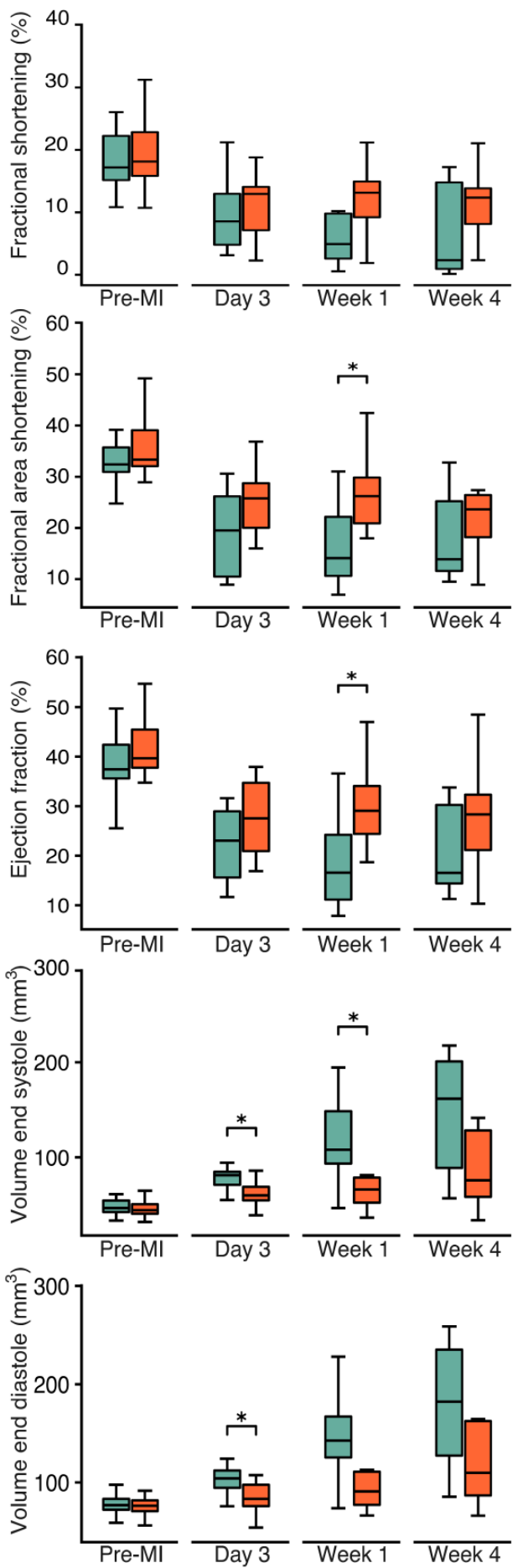

Figure 4: Echocardiographic assessment of hemodynamic parameters in wild-type and STAT1F77A knock-in mice. Before LAD ligation ( $n=23$ and $n=23$, respectively), 3 days, $(n=12$ versus $n=12)$, one week ( $n=9$ versus $n=8)$ and four weeks $(n=7$ versus $n=8)$ following LAD ligation. ${ }^{*} p \leq$ 0.05 by independent-samples $t$-test. 
Table 20: Measurements of important structural cardiac parameters in wild-type and STAT1F77A knock-in mice.

\begin{tabular}{|c|c|c|c|c|c|c|c|c|c|}
\hline & Time & Genotype & Mean (CI) & Min & Q1 & Median & Q3 & Max & $p$-value \\
\hline \multirow{8}{*}{ 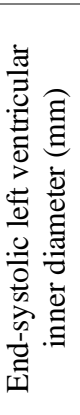 } & \multirow[t]{2}{*}{ Pre-MI } & WT & $3.50(3.38-3.63)$ & 3.15 & 3.26 & 3.47 & 3.73 & 4.15 & \multirow[t]{2}{*}{0.99} \\
\hline & & STAT1-F77A & $3.47(3.34-3.59)$ & 2.76 & 3.3 & 3.5 & 3.68 & 3.98 & \\
\hline & \multirow[t]{2}{*}{ Day 3} & WT & $4.39(4.15-4.64)$ & 3.58 & 4.19 & 4.4 & 4.51 & 5.03 & \multirow[t]{2}{*}{$0.036 *$} \\
\hline & & STAT1-F77A & $3.92(3.59-4.25)$ & 2.95 & 3.68 & 3.79 & 4.25 & 4.71 & \\
\hline & \multirow[t]{2}{*}{ Week 1} & WT & $5.40(4.6-6.20)$ & 3.42 & 5.05 & 5.51 & 6.35 & 6.68 & \multirow[t]{2}{*}{$0.049 *$} \\
\hline & & STAT1-F77A & $4.38(3.7-5.05)$ & 3.43 & 3.72 & 4.3 & 4.86 & 5.86 & \\
\hline & \multirow[t]{2}{*}{ Week 4} & WT & $5.55(4.26-6.83)$ & 3.83 & 4.32 & 5.82 & 6.75 & 7.03 & \multirow[t]{2}{*}{0.52} \\
\hline & & STAT1-F77A & $4.94(3.75-6.13)$ & 3.4 & 4.03 & 4.64 & 5.35 & 7.94 & \\
\hline \multirow{8}{*}{ 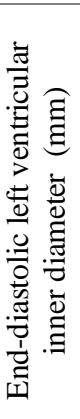 } & \multirow[t]{2}{*}{ Pre-MI } & WT & $4.29(4.18-4.4)$ & 3.83 & 4.14 & 4.25 & 4.39 & 5.01 & \multirow[t]{2}{*}{0.52} \\
\hline & & STAT1-F77A & $4.31(4.22-4.4)$ & 3.94 & 4.19 & 4.35 & 4.46 & 4.62 & \\
\hline & \multirow[t]{2}{*}{ Day 3} & WT & $4.79(4.64-4.94)$ & 4.33 & 4.68 & 4.76 & 4.94 & 5.19 & \multirow[t]{2}{*}{$0.0035^{*}$} \\
\hline & & STAT1-F77A & $4.39(4.14-4.64)$ & 3.46 & 4.25 & 4.56 & 4.62 & 4.82 & \\
\hline & \multirow[t]{2}{*}{ Week 1} & WT & $5.60(5.05-6.16)$ & 4.36 & 5.33 & 5.66 & 6.09 & 6.86 & \multirow[t]{2}{*}{0.061} \\
\hline & & STAT1-F77A & $4.96(4.43-5.49)$ & 4.18 & 4.34 & 5.06 & 5.36 & 5.98 & \\
\hline & \multirow[t]{2}{*}{ Week 4} & WT & $5.9(4.94-6.85)$ & 4.57 & 5.1 & 5.83 & 6.8 & 7.09 & \multirow[t]{2}{*}{0.45} \\
\hline & & STAT1-F77A & $5.47(4.52-6.43)$ & 4.31 & 4.59 & 5.39 & 5.93 & 7.76 & \\
\hline \multirow{8}{*}{ 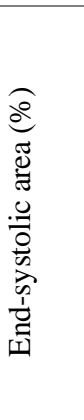 } & \multirow[t]{2}{*}{ Pre-MI } & WT & $8.28(7.68-8.88)$ & 6.43 & 7.34 & 8.2 & 8.98 & 11.96 & \multirow[t]{2}{*}{0.38} \\
\hline & & STAT1-F77A & $7.92(7.32-8.52)$ & 5.77 & 6.97 & 7.8 & 8.54 & 11.55 & \\
\hline & \multirow[t]{2}{*}{ Day 3} & WT & $12.79(11.54-14.05)$ & 9.1 & 11.64 & 12.93 & 14.17 & 15.91 & \multirow[t]{2}{*}{$0.0068 *$} \\
\hline & & STAT1-F77A & $10.24(8.92-11.55)$ & 6.64 & 9.27 & 10.09 & 11.11 & 13.27 & \\
\hline & \multirow[t]{2}{*}{ Week 1} & WT & $18.09(13.54-22.65)$ & 8.76 & 14.42 & 17.14 & 21.62 & 27.96 & $0.030 *$ \\
\hline & & STAT1-F77A & $11.55(7.79-15.31)$ & 6.17 & 8.83 & 11.7 & 12.22 & 21.12 & \\
\hline & Week 4 & WT & $20.64(12.79-28.50)$ & 9.18 & 13.38 & 23.19 & 28.01 & 29.35 & 0.38 \\
\hline & & STAT1-F77A & $16.43(7.76-25.10)$ & 6.51 & 9.63 & 14.18 & 18.19 & 39.39 & \\
\hline & Pre-MI & WT & $12.2(11.6-12.81)$ & 10.01 & 11.2 & 12.27 & 12.79 & 15.89 & 0.95 \\
\hline$\widetilde{\tau}$ & & STAT1-F77A & $12.22(11.55-12.90)$ & 9.47 & 11.56 & 12.01 & 12.96 & 16.62 & \\
\hline$\Xi$ & Day 3 & WT & $15.83(14.81-16.85)$ & 12.3 & 15.52 & 16.34 & 16.79 & 17.81 & $0.013 *$ \\
\hline 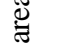 & & STAT1-F77A & $13.57(12.17-14.98)$ & 9 & 12.39 & 13.63 & 15 & 16.49 & \\
\hline$\because \frac{0}{0}$ & Week 1 & WT & $21.29(16.79-25.79)$ & 12.7 & 19.4 & 22.02 & 24.2 & 32.24 & 0.061 \\
\hline 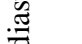 & & STAT1-F77A & $15.54(11.5-19.58)$ & 10.72 & 12.13 & 15.15 & 16.6 & 25.74 & \\
\hline ت્己 & Week 4 & WT & $24.68(17.06-32.31)$ & 12.34 & 18.73 & 25.62 & 32.24 & 32.91 & 0.27 \\
\hline & & STAT1-F77A & $20.52(11.86-29.19)$ & 11.58 & 12.95 & 19.07 & 22.71 & 43.23 & \\
\hline & Pre-MI & WT & $18.19(17.4-18.98)$ & 15.93 & 16.73 & 17.34 & 19.23 & 22.66 & 0.29 \\
\hline$\Xi$ & & STAT1-F77A & $18.61(17.78-19.44)$ & 14.57 & 17.71 & 18.4 & 19.58 & 23.67 & \\
\hline $\mathbb{J}$ & Day 3 & WT & $25.02(23.57-26.47)$ & 20.73 & 23.83 & 24.75 & 26.35 & 28.62 & 0.17 \\
\hline .0 & & STAT1-F77A & $23.21(20.18-26.24)$ & 14.12 & 21.04 & 22.91 & 25.33 & 31.5 & \\
\hline$\frac{0}{0}$ & Week 1 & WT & $29.52(24.32-34.71)$ & 20.27 & 25.03 & 29.1 & 32.28 & 41.8 & $0.039 *$ \\
\hline$\frac{\infty}{\pi}$ & & STAT1-F77A & $22.6(18.36-26.84)$ & 17.17 & 18.47 & 23.15 & 24.22 & 32.66 & \\
\hline 氖 & Week 4 & WT & $34.49(26.27-42.72)$ & 21.26 & 27.83 & 38.71 & 40.83 & 44.16 & 0.15 \\
\hline जิ & & STAT1-F77A & $28.97(17.9-40.04)$ & 17.07 & 20.87 & 25.86 & 30.56 & 59.27 & \\
\hline
\end{tabular}

Before MI ( $n=23$ and $n=23$, respectively), and at different time points after MI: 3 days, $(n=12$ versus $n=12)$, one week $(n=9$ versus $n=8)$ and four weeks $(n=7$ versus $n=8)$. Mean, lower and upper quartile of the mean, minimum, first (Q1) and third (Q3) quartiles, median, and maximum are shown below. 
Table 21: Assessment of important functional cardiac parameters in wild-type and STAT1-

F77A knock-in mice

\begin{tabular}{|c|c|c|c|c|c|c|c|c|c|}
\hline & Time & Genotype & Mean (CI) & Min & Q1 & Median & Q3 & Max & $p$-value \\
\hline \multirow{8}{*}{ 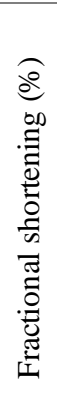 } & \multirow[t]{2}{*}{ Pre-MI } & WT & $18.35(16.43-20.28)$ & 10.84 & 15.16 & 17.19 & 22.27 & 26.06 & \multirow[t]{2}{*}{0.49} \\
\hline & & STAT1-F77A & $19.54(17.19-21.90)$ & 10.74 & 15.84 & 18.14 & 22.84 & 31.27 & \\
\hline & \multirow[t]{2}{*}{ Day 3} & WT & $9.47(5.85-13.08)$ & 3.11 & 4.81 & 8.56 & 12.98 & 21.21 & \multirow[t]{2}{*}{0.56} \\
\hline & & STAT1-F77A & $10.88(7.06-14.71)$ & 2.28 & 7.13 & 12.96 & 14.1 & 18.8 & \\
\hline & \multirow[t]{2}{*}{ Week 1} & WT & $6.79(1.82-11.77)$ & 0.55 & 2.58 & 4.93 & 9.81 & 21.67 & \multirow[t]{2}{*}{0.11} \\
\hline & & STAT1-F77A & $12.23(7.50-16.95)$ & 1.88 & 9.22 & 13.13 & 14.95 & 21.19 & \\
\hline & \multirow[t]{2}{*}{ Week 4} & WT & $7.31(0.02-14.6)$ & 0.13 & 0.93 & 2.34 & 14.81 & 17.24 & \multirow[t]{2}{*}{0.35} \\
\hline & & STAT1-F77A & $11.38(6.56-16.19)$ & 2.34 & 8.13 & 12.38 & 13.84 & 21.07 & \\
\hline \multirow{8}{*}{ 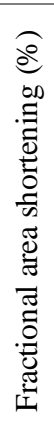 } & \multirow[t]{2}{*}{ Pre-MI } & WT & $32.38(29.86-34.91)$ & 21.25 & 30.93 & 32.38 & 35.73 & 45.46 & \multirow[t]{2}{*}{0.22} \\
\hline & & STAT1-F77A & $35.26(32.23-38.29)$ & 18.62 & 32.04 & 33.33 & 39.07 & 49.9 & \\
\hline & \multirow[t]{2}{*}{ Day 3} & WT & $19.33(14.07-24.58)$ & 8.9 & 10.51 & 19.53 & 26.17 & 30.58 & \multirow[t]{2}{*}{0.21} \\
\hline & & STAT1-F77A & $24.72(20.16-29.29)$ & 15.97 & 20.02 & 25.76 & 28.74 & 36.84 & \\
\hline & \multirow[t]{2}{*}{ Week 1} & WT & $16.13(9.7-22.56)$ & 6.98 & 10.65 & 14.09 & 22.14 & 31.01 & \multirow[t]{2}{*}{$0.024 *$} \\
\hline & & STAT1-F77A & $26.92(20.36-33.48)$ & 17.96 & 20.88 & 26.2 & 29.84 & 42.42 & \\
\hline & \multirow[t]{2}{*}{ Week 4} & WT & $18.52(10.15-26.9)$ & 9.49 & 11.6 & 13.86 & 25.2 & 32.73 & \multirow[t]{2}{*}{0.45} \\
\hline & & STAT1-F77A & $23.29(14.49-32.08)$ & 8.89 & 18.18 & 23.62 & 26.43 & 43.81 & \\
\hline \multirow{8}{*}{ 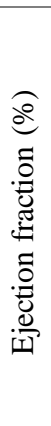 } & \multirow[t]{2}{*}{ Pre-MI } & WT & $37.82(35.23-40.41)$ & 25.52 & 35.6 & 37.46 & 42.4 & 49.68 & \multirow[t]{2}{*}{0.11} \\
\hline & & STAT1-F77A & $41.08(38.23-43.93)$ & 25.37 & 37.76 & 39.65 & 45.43 & 54.71 & \\
\hline & \multirow[t]{2}{*}{ Day 3} & WT & $22.27(17.45-27.08)$ & 11.65 & 15.57 & 23.04 & 28.93 & 31.61 & \multirow[t]{2}{*}{0.10} \\
\hline & & STAT1-F77A & $27.39(22.25-32.54)$ & 16.9 & 20.91 & 27.53 & 34.71 & 37.89 & \\
\hline & \multirow[t]{2}{*}{ Week 1} & WT & $18.60(11.26-25.95)$ & 7.84 & 11.14 & 16.59 & 24.23 & 36.59 & $0.030 *$ \\
\hline & & STAT1-F77A & $30.11(22.64-37.57)$ & 18.69 & 24.41 & 29.05 & 34.08 & 47 & \\
\hline & Week 4 & WT & $21.55(12.64-30.46)$ & 11.25 & 14.41 & 16.54 & 30.26 & 33.76 & 0.52 \\
\hline & & STAT1-F77A & $27.36(17.33-37.38)$ & 10.31 & 21.11 & 28.32 & 32.31 & 48.46 & \\
\hline & Pre-MI & WT & $48.50(44.3-52.7)$ & 33.58 & 42.47 & 46.68 & 54.67 & 75.21 & 0.25 \\
\hline$\stackrel{m}{\Xi}$ & & STAT1-F77A & $45.08(41.36-48.8)$ & 32.28 & 40.73 & 44.56 & 50.86 & 64.85 & \\
\hline$=$ & Day 3 & WT & $79.49(69.93-89.05)$ & 55.23 & 71.23 & 81.4 & 85.01 & 108.4 & $0.039 *$ \\
\hline$\Xi$ & & STAT1-F77A & $62.44(52.42-72.45)$ & 39.05 & 54.82 & 60.11 & 69.01 & 86 & \\
\hline . & Week 1 & WT & $119.69(84.14-155.23)$ & 46.72 & 93.47 & 108.07 & 148.48 & 194.47 & $0.039 *$ \\
\hline 营 & & STAT1-F77A & $72.00(42.88-101.12)$ & 36.61 & 52.32 & 66.16 & 78.59 & 148.88 & \\
\hline$\stackrel{1}{a}_{=}^{1}$ & Week 4 & WT & $145.13(83.69-206.57)$ & 57.03 & 88.86 & 161.71 & 200.87 & 217.71 & 0.27 \\
\hline II & & STAT1-F77A & $108.43(37.11-179.76)$ & 34.04 & 58.41 & 75.94 & 128.26 & 299.99 & \\
\hline & Pre-MI & WT & $77.69(72.7-82.68)$ & 59.02 & 72.24 & 76.89 & 83.34 & 110.86 & 0.78 \\
\hline m. & & STAT1-F77A & $76.46(71.59-81.32)$ & 56.03 & 70.81 & 76.27 & 81.72 & 106.57 & \\
\hline 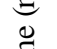 & Day 3 & WT & $101.9(93.03-110.78)$ & 75.72 & 94.42 & 104.22 & 112.07 & 124.1 & $0.045^{*}$ \\
\hline$\Xi$ & & STAT1-F77A & $85.82(74.52-97.12)$ & 53.89 & 75.85 & 83.24 & 97.68 & 107.4 & \\
\hline. & Week 1 & WT & $143.94(108.35-179.53)$ & 73.67 & 125.49 & 142.63 & 167.09 & 228.05 & 0.061 \\
\hline$\frac{0}{3}$ & & STAT1-F77A & $100.36(68.71-132.01)$ & 66.42 & 77.08 & 90.74 & 110.85 & 183.09 & \\
\hline$i_{i}^{i}$ & Week 4 & WT & $178.84(116.48-241.2)$ & 85.47 & 127.35 & 182.2 & 235.29 & 258.94 & 0.22 \\
\hline 피 & & STAT1-F77A & $139.31(66.55-212.07)$ & 66.05 & 86.83 & 109.76 & 162.51 & 334.46 & \\
\hline
\end{tabular}

Before $M I$ ( $n=23$ and $n=23$, respectively), and at different time points after MI: 3 days, $(n=12$ versus $n=12)$, one week ( $n=9$ versus $n=8)$ and four weeks $(n=7$ versus $n=8)$. Mean, lower and upper quartile of the mean, minimum, first (Q1) and third (Q3) quartiles, median, and maximum are shown below. 


\subsection{Expression of STAT1 and STAT3 in both cardiomyocytes and infiltrating immune}

\section{cells after myocardial infarction}

In order to analyze the expression profile of STAT1 and STAT3 proteins, immunohistochemical staining was performed in sections of infarcted area for STAT1-F77A and wild-type mice undergoing LAD ligation or sham operation. Immunohistochemical analysis of staining intensity and distribution revealed expression of STAT1 and STAT3 in both cardiomyocytes and infiltrating immune cells at day 3 post-MI, while no significant differences in their expression levels were identified among the two groups (Figure 5).
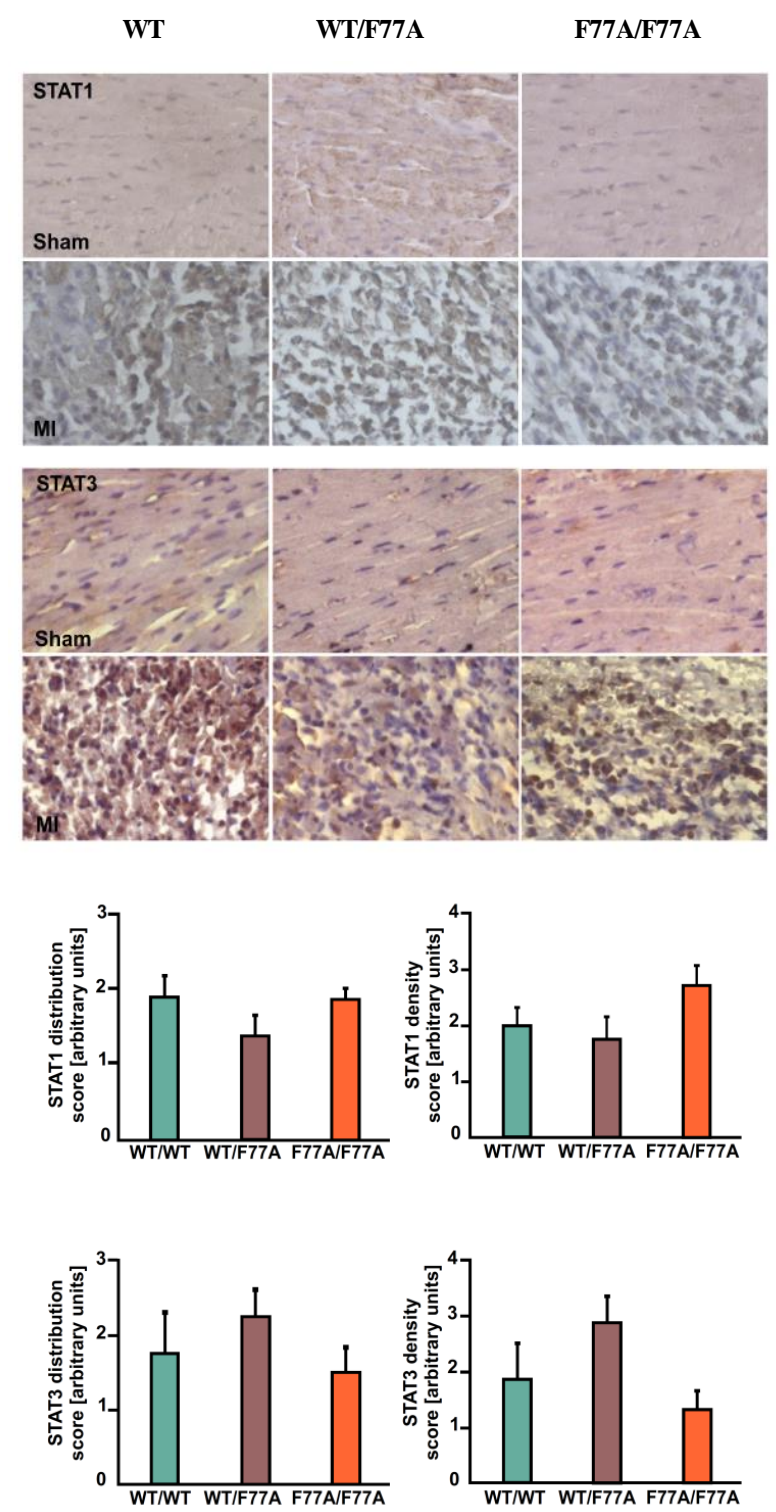

Figure 5: Immunohistochemical analysis of STAT1 and STAT3 expression in the infarcted area at day 3 post-MI. Semi-quantitative analysis of STAT staining distribution and intensity are presented as means $\pm S D$. 


\subsection{Transcriptomic analysis of F77A mice in a LAD ligation model}

With the aim to analyze the left ventricular global transcriptional profile, we constructed 12 RNA libraries and sequenced them (Figure 6). Samples were collected from sham-operated or LAD-ligation-operated mice expressing either wild-type or mutant STAT1. The quality controls as well as the number of pooled mice for extracted RNA are shown in Table 22 and Figure 7.

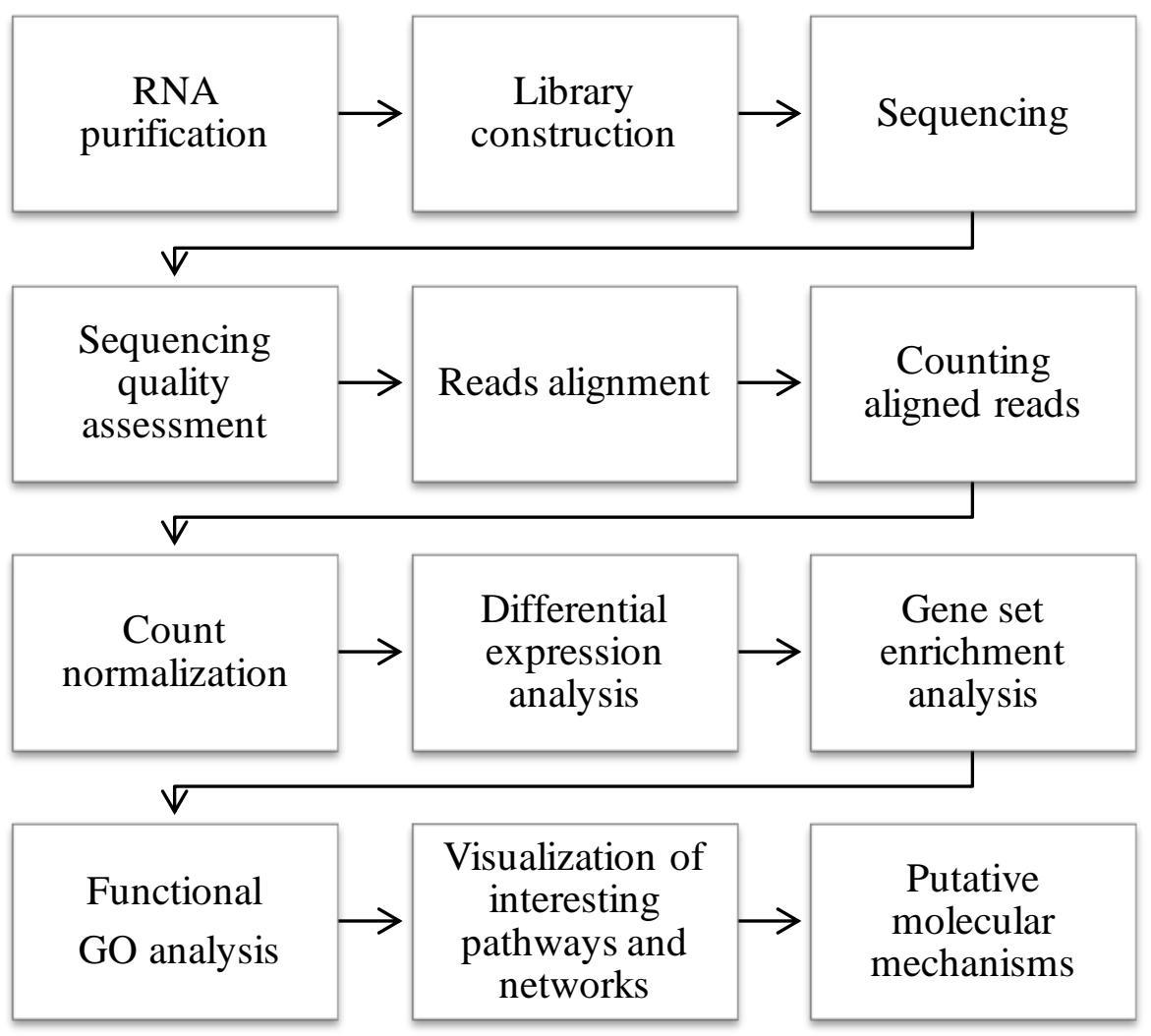

Figure 6: RNA-seq pipeline to establish transcriptomic signature of the infarcted area. 
Table 22: Quality assessment of total RNA in the infarcted heart tissue samples.

\begin{tabular}{|c|c|c|c|c|c|c|c|}
\hline Sample ID & $\mathbf{N}$ & $\begin{array}{c}\text { Concentration } \\
(\mathbf{n g} / \mu \mathrm{l})\end{array}$ & Yield $(\mu \mathrm{g})$ & A260/A280 & A260/A230 & RIN & $28 s / 18 s$ \\
\hline WT-Sham-1 & 4 & $711.46 \pm 197.94$ & $35.57 \pm 9.9$ & $1.92 \pm 0.05$ & $1.61 \pm 0.33$ & $9.35 \pm 0.16$ & $1.40 \pm 0.19$ \\
\hline WT-Sham-2 & 4 & $728.99 \pm 68.55$ & $36.45 \pm 3.43$ & $1.88 \pm 0.04$ & $1.35 \pm 0.28$ & $9.88 \pm 0.08$ & $1.33 \pm 0.22$ \\
\hline WT-Sham-3 & 3 & $910.82 \pm 130.15$ & $45.54 \pm 6.51$ & $1.97 \pm 0.03$ & $1.73 \pm 0.33$ & $10.00 \pm 0$ & $1.43 \pm 0.29$ \\
\hline WT-MI-1 & 4 & $341.19 \pm 59.23$ & $17.06 \pm 2.96$ & $1.87 \pm 0.01$ & $2.08 \pm 0.07$ & $8.35 \pm 0.28$ & $1.65 \pm 0.27$ \\
\hline WT-MI-2 & 4 & $524.07 \pm 80.14$ & $26.20 \pm 4.01$ & $1.90 \pm 0.02$ & $1.66 \pm 0.25$ & $9.45 \pm 0.06$ & $1.73 \pm 0.2$ \\
\hline WT-MI-3 & 3 & $348.60 \pm 45.23$ & $17.43 \pm 2.26$ & $1.83 \pm 0.01$ & $2.01 \pm 0.06$ & $9.87 \pm 0.13$ & $1.67 \pm 0.09$ \\
\hline F77A-Sham-1 & 2 & $493.65 \pm 12.11$ & $24.68 \pm 0.61$ & $1.89 \pm 0.07$ & $1.54 \pm 0.52$ & $9.00 \pm 0.6$ & $1.40 \pm 0$ \\
\hline F77A-Sham-2 & 1 & 435.19 & 21.76 & 1.88 & 2.19 & 10.00 & 1.20 \\
\hline F77A-Sham-3 & 1 & 768.92 & 38.45 & 1.95 & 1.44 & 10.00 & 1.70 \\
\hline F77A-MI-1 & 4 & $316.85 \pm 59.01$ & $15.84 \pm 2.95$ & $1.86 \pm 0.01$ & $1.70 \pm 0.24$ & $8.03 \pm 0.08$ & $1.35 \pm 0.05$ \\
\hline F77A-MI-2 & 3 & $331.88 \pm 82.68$ & $16.59 \pm 4.13$ & $1.80 \pm 0.06$ & $1.61 \pm 0.51$ & $8.23 \pm 0.03$ & $1.27 \pm 0.09$ \\
\hline F77A-MI-3 & 3 & $378.46 \pm 114.19$ & $18.92 \pm 5.71$ & $1.88 \pm 0.01$ & $1.63 \pm 0.25$ & $8.67 \pm 0.09$ & $2.80 \pm 1.55$ \\
\hline
\end{tabular}
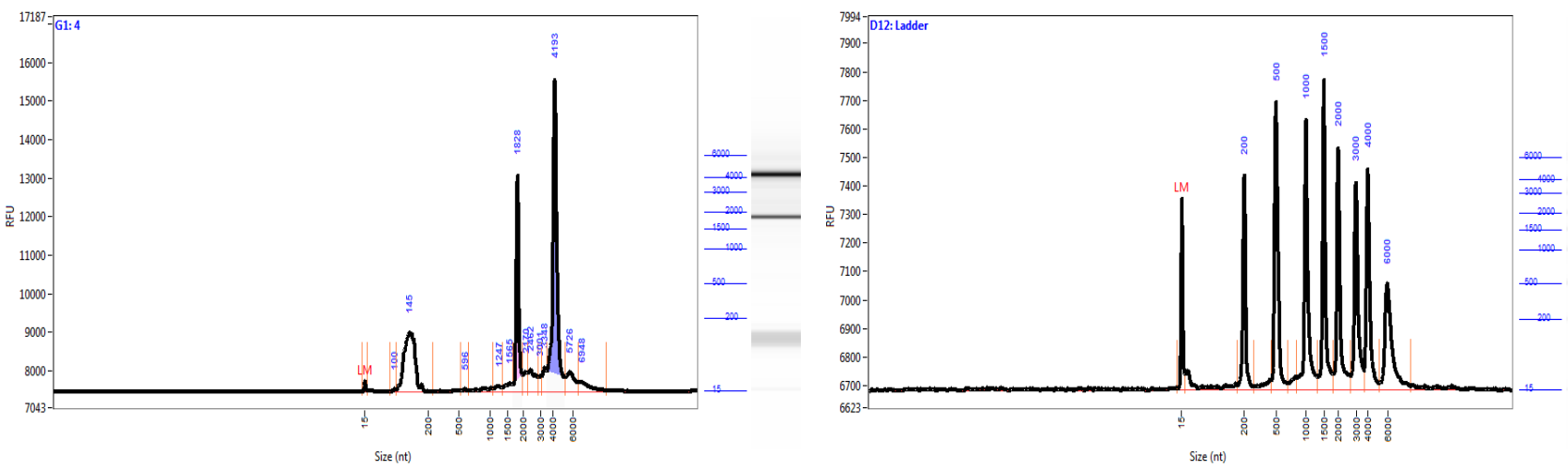

Figure 7: Separation of high-quality total RNA extracted from infarcted heart tissue. A) The purple and pink peaks represent the large (28S rRNA) and small (18S rRNA) ribosomal RNA peaks, respectively. The small RNA region includes the peaks and smears below 200 nucleotides, which is well defined, as these are the small and large rRNA subunits, providing a high RQN (RNA quality number) of 10. All samples selected for sequencing exhibited an RNA integrity number over 8. B) Separation of HS RNA Ladder on a Fragment Analyzer using the Standard Sensitivity RNA analysis kit (DNF-471). 


\subsection{Identification of differentially expressed genes in STAT1-F77A and wild-type mice at} day 1 post-myocardial infarction

To identify differentially expressed genes (DEGs) following MI in wild-type and mutant mice, a $\mid \log _{2}$ (fold change) $\mid>1$ and a $p$-value $<0.05$ were used as standards. We identified 1912 differentially expressed genes in wild-type mice. However, as a consequence of LAD ligation, the number of differentially regulated genes was 4055 genes in STAT1-F77A, which is significantly higher as compared to wild-type mice. Furthermore, subsequent to MI induction, we found 194 DEGs that were unique in wild-type mice but were not significantly modulated in STAT1-F77A mice, and 2337 DEGs that are unique in STAT1-F77A mice, but not changed in wild-type mice post-MI. Moreover, we identified 1718 DEGs that were commonly expressed in wild-type and STAT1-F77A mice. Of the 2337 uniquely differentially expressed genes in the STAT1-F77A following MI, there were 909 upregulated and 1428 downregulated genes, whereas, of the 194 uniquely differentially expressed genes in wild-type animals, there were 111 upregulated and 83 downregulated genes (Figure 8). The hierarchical clustering of RPKM (reads per kilobase of transcript per million mapped reads) values of the differentially expressed genes for both wild-type (MI vs sham) and STAT1F77A (MI vs sham) comparisons in the four groups (wild-type sham, wild-type MI, STAT1F77A sham, STAT1-F77A MI) are shown in Figure 9.

The top 10 genes that are differentially expressed in wild-type following MI are the following: Hspala (heat shock protein family a (hsp70) member 1a), Hspalb (heat shock protein family a (hsp70) member 1b), Fgf23 (fibroblast growth factor 23), Cxcl2 (C-X-C motif chemokine ligand 2), Ngp (neutrophilic granule protein), Fosb (fosb proto-oncogene, ap-1 transcription factor subunit), Aa467197 (expressed sequence aa467197), Cxcl10 (C-X-C motif chemokine ligand 10), Ccl4 (c-c motif chemokine ligand 4), Cd177 (human neutrophil alloantigen 2a). Table 23 illustrates the top 20 differentially expressed genes in wild-type (LAD ligation vs sham) comparison with the $\log _{2}$ fold change, padj, $\log _{10}$ padj and baseMean values shown. 


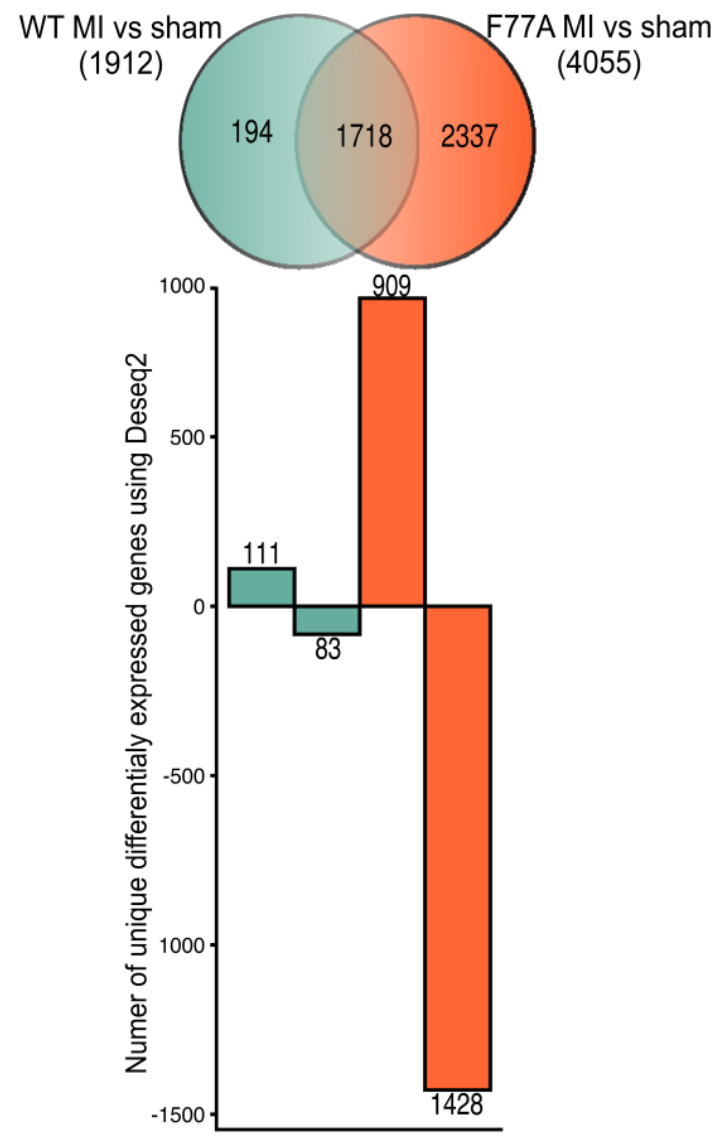

Figure 8: The number of unique genes that are upregulated or downregulated in wild-type and STAT1-F77A following LAD ligation.

Table 23: Top 20 genes differentially expressed in wild-type following LAD ligation

\begin{tabular}{lllll}
\hline Gene & $\log _{2}$ fold change & padj & $\log _{10}$ padj & baseMean \\
\hline Hspala & 3.77 & $9.56 \mathrm{e}-11$ & $1.00 \mathrm{e}+01$ & 1851.08 \\
\hline Hspalb & 3.66 & $4.59 \mathrm{e}-10$ & $9.34 \mathrm{e}+00$ & 2979.38 \\
\hline Fgf23 & 3.65 & $1.91 \mathrm{e}-10$ & $9.72 \mathrm{e}+00$ & 216.89 \\
\hline Cxcl2 & 3.62 & $4.60 \mathrm{e}-11$ & $1.03 \mathrm{e}+01$ & 1167.84 \\
\hline Ngp & 3.43 & $2.40 \mathrm{e}-08$ & $7.62 \mathrm{e}+00$ & 49.94 \\
\hline Fosb & 3.39 & $1.53 \mathrm{e}-08$ & $7.82 \mathrm{e}+00$ & 478.94 \\
\hline AA467197 & 3.31 & $4.37 \mathrm{e}-08$ & $7.36 \mathrm{e}+00$ & 690.60 \\
\hline Cxcl10 & 3.28 & $3.62 \mathrm{e}-09$ & $8.44 \mathrm{e}+00$ & 422.35 \\
\hline Ccl4 & 3.27 & $1.02 \mathrm{e}-09$ & $8.99 \mathrm{e}+00$ & 393.55 \\
\hline Cd177 & 3.25 & $1.28 \mathrm{e}-09$ & $8.89 \mathrm{e}+00$ & 406.20 \\
\hline Thbs1 & 3.24 & $5.26 \mathrm{e}-11$ & $1.03 \mathrm{e}+01$ & 12485.02 \\
\hline Hcar2 & 3.23 & $1.46 \mathrm{e}-08$ & $7.84 \mathrm{e}+00$ & 337.96 \\
\hline Cxcl3 & $1.20 \mathrm{e}-08$ & $7.92 \mathrm{e}+00$ & 1422.77 \\
\hline Asprv1 & 3.21 & $6.28 \mathrm{e}-11$ & $1.02 \mathrm{e}+01$ & 290.54 \\
\hline Ptgs2 & 3.21 & $3.82 \mathrm{e}-08$ & $7.42 \mathrm{e}+00$ & 1411.21 \\
\hline Slc7al1 & 3.18 & $1.36 \mathrm{e}-08$ & $7.87 \mathrm{e}+00$ & 422.98 \\
\hline Retnlg & 3.17 & $1.20 \mathrm{e}-11$ & $1.09 \mathrm{e}+01$ & 797.23 \\
\hline Il6 & 3.16 & $7.46 \mathrm{e}-09$ & $8.13 \mathrm{e}+00$ & 532.09 \\
\hline Selp & 3.16 & $5.26 \mathrm{e}-11$ & $1.03 \mathrm{e}+01$ & 1177.32 \\
\hline Illr2 & 3.16 & $1.98 \mathrm{e}-08$ & $7.70 \mathrm{e}+00$ & 1517.34 \\
\hline
\end{tabular}


In addition, the top 10 genes that are differentially expressed in STAT1-F77A (LAD ligation vs sham) comparison are the following: Fgf23 (fibroblast growth factor 23), Cxcl3 (C-X-C motif chemokine ligand 3), Slfn4 (schlafen 4), Rab44 (rab44, member ras oncogene family), Cxcl2 (C-X-C motif chemokine ligand 2), Cd177 (human neutrophil alloantigen 2a), Gm5483 (predicted gene 5483), Illr2 (interleukin 1 receptor type 2), Cd300lf (cd300 molecule like family member f), Slfnl (schlafen family member 12). Table 24 illustrates the top 20 differentially expressed genes in STAT1-F77A following LAD ligation with the $\log _{2}$ fold change, padj, $\log _{10}$ padj and baseMean values shown.

Table 24: Top 20 genes differentially expressed in STAT1-F77A following LAD ligation

\begin{tabular}{lrllr}
\hline Gene & $\log _{2}$ fold change & padj & $\log _{10}$ padj & baseMean \\
\hline Fgf23 & 4.89 & $1.29 \mathrm{e}-19$ & $1.89 \mathrm{e}+01$ & 216.89 \\
\hline Cxcl3 & 4.76 & $9.07 \mathrm{e}-20$ & $1.90 \mathrm{e}+01$ & 1422.77 \\
\hline Slfn4 & 4.64 & $8.59 \mathrm{e}-21$ & $2.01 \mathrm{e}+01$ & 5478.06 \\
\hline Rab44 & 4.52 & $1.37 \mathrm{e}-17$ & $1.69 \mathrm{e}+01$ & 788.41 \\
\hline Cxcl2 & 4.51 & $1.38 \mathrm{e}-18$ & $1.79 \mathrm{e}+01$ & 1167.84 \\
\hline Cd177 & 4.49 & $3.60 \mathrm{e}-19$ & $1.84 \mathrm{e}+01$ & 406.20 \\
\hline Gm5483 & 4.45 & $1.53 \mathrm{e}-16$ & $1.58 \mathrm{e}+01$ & 136.50 \\
\hline Illr2 & 4.41 & $2.53 \mathrm{e}-17$ & $1.66 \mathrm{e}+01$ & 1517.34 \\
\hline Cd300lf & 4.40 & $4.25 \mathrm{e}-22$ & $2.14 \mathrm{e}+01$ & 1932.80 \\
\hline Slfn1 & 4.34 & $9.07 \mathrm{e}-20$ & $1.90 \mathrm{e}+01$ & 1233.29 \\
\hline Ptgs 2 & 4.27 & $2.51 \mathrm{e}-15$ & $1.46 \mathrm{e}+01$ & 1411.21 \\
\hline Cxcr2 & 4.19 & $3.23 \mathrm{e}-18$ & $1.75 \mathrm{e}+01$ & 1119.46 \\
\hline Slc7a11 & 4.19 & $9.62 \mathrm{e}-16$ & $1.50 \mathrm{e}+01$ & 422.98 \\
\hline Hcar2 & 4.18 & $5.19 \mathrm{e}-15$ & $1.43 \mathrm{e}+01$ & 337.96 \\
\hline Sh2d5 & 4.15 & $1.04 \mathrm{e}-13$ & $1.30 \mathrm{e}+01$ & 925.10 \\
\hline Adamts4 & 4.13 & $5.50 \mathrm{e}-14$ & $1.33 \mathrm{e}+01$ & 3382.60 \\
\hline Trem1 & 4.13 & $2.77 \mathrm{e}-17$ & $1.66 \mathrm{e}+01$ & 631.05 \\
\hline Hspala & 4.12 & $5.50 \mathrm{e}-14$ & $1.33 \mathrm{e}+01$ & 1851.08 \\
\hline Mirt2 & 4.12 & $4.52 \mathrm{e}-13$ & $1.23 \mathrm{e}+01$ & 69.10 \\
\hline Il6 & 4.09 & $1.18 \mathrm{e}-15$ & $1.49 \mathrm{e}+01$ & 532.09 \\
\hline
\end{tabular}


DE genes in WT MI vs sham

DE genes in F77A MI vs sham
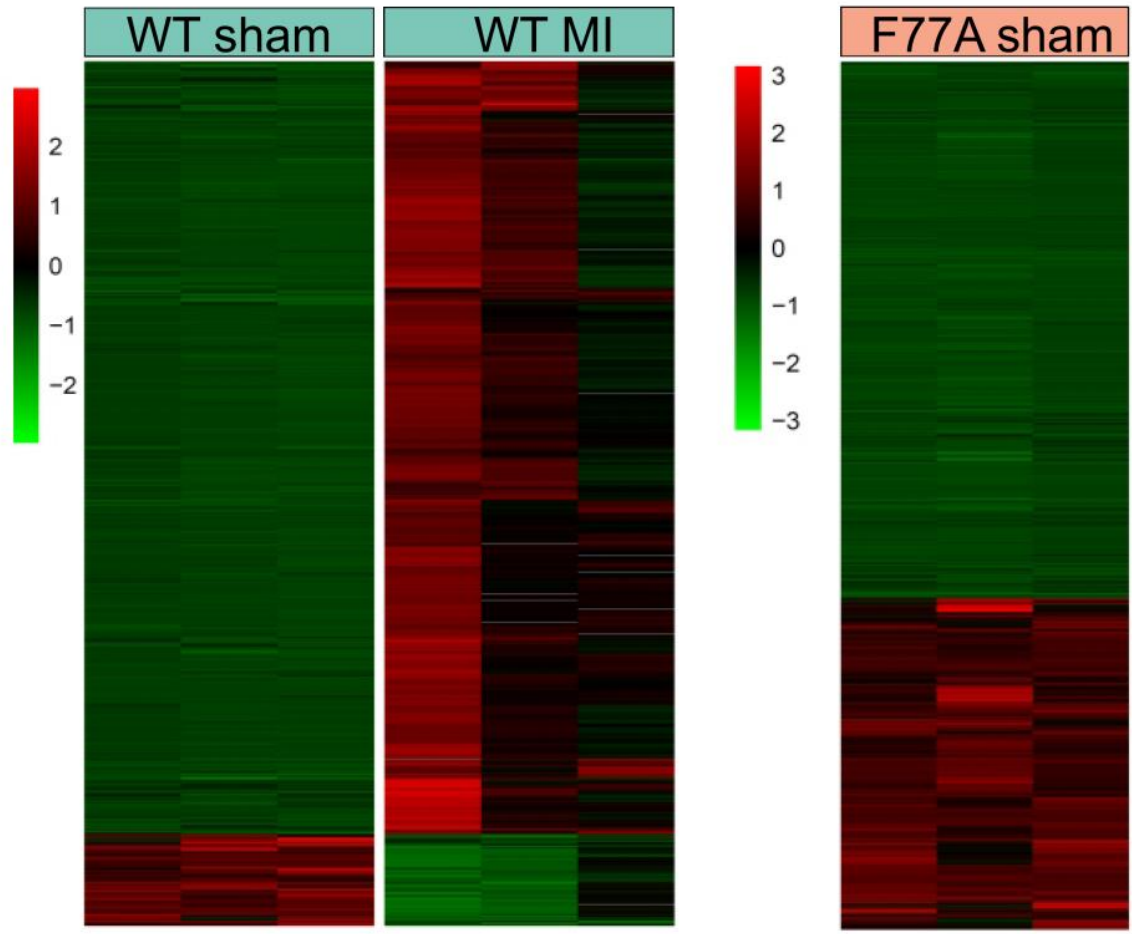

\section{F77A MI}
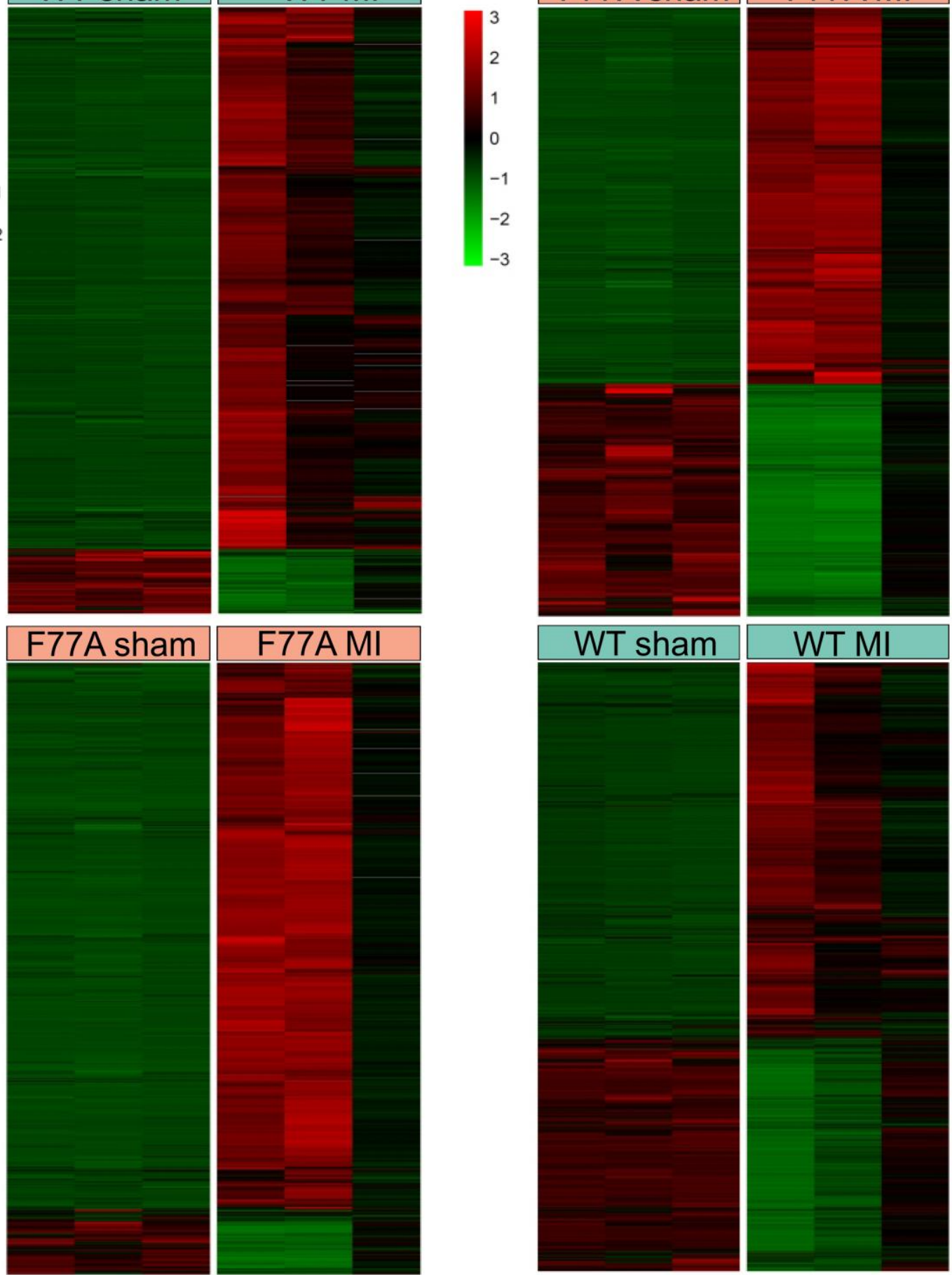

Figure 9: Hierarchical clustering of RPKM values in wild-type and STAT1-F77A mice in both sham-operated and LAD-ligation-operated mice. The first heat map was generated using the list of genes that are differentially expressed in wild-type (MI vs sham) comparison (in total 1912 genes). The second heat map was generated using genes that are differentially expressed in STAT1-F77A (MI vs sham) comparison (in total 4055 genes). 
To depict the distribution of differentially regulated genes unique in unaffected myocardium and infarcted areas from the hearts of wild-type and STAT1-F77A mice, a Venn diagram representation was used. Figure 10 demonstrates the number of differentially expressed genes found in the following four groups: wild-type (MI vs sham) upregulated, wild-type (MI vs sham) downregulated, STAT1-F77A (MI vs sham) upregulated, and STAT1-F77A (MI vs sham) downregulated. The overlap of the two ovals in the top of the diagram is 1598, which is the number of differentially expressed genes that are shared between wild-type (MI vs sham) upregulated and F77A (MI vs sham) upregulated lists. The overlap of the two ovals in the bottom of the diagram is 120 , which is the number of the differentially expressed genes that are shared between the wild-type (MI vs sham) downregulated and F77A (MI vs sham) downregulated lists. The areas of no overlap represent the number of uniquely (not shared) differentially expressed genes in each respective comparison.

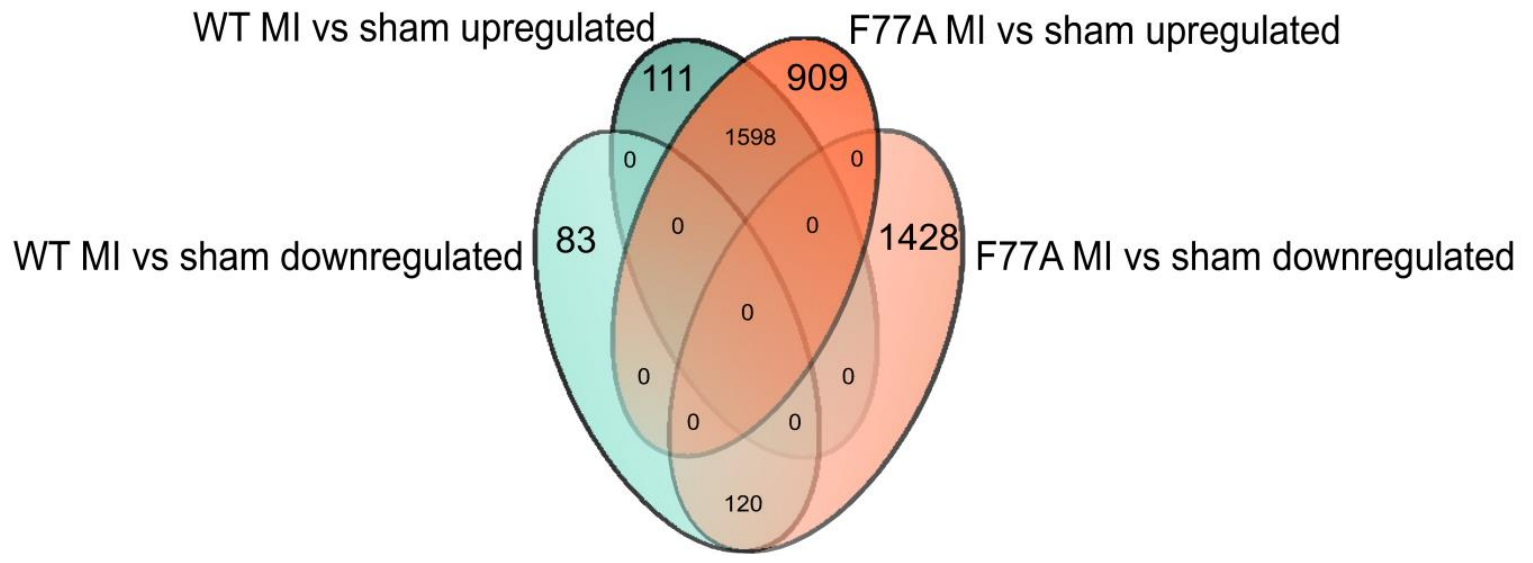

Figure 10: The numbers of differentially expressed genes in the infarcted area common and unique in wild-type and STAT1-F77A mice. The following pairwise comparisons are shown: wildtype (MI vs sham) upregulated, wild-type (MI vs sham) downregulated, STAT1-F77A (MI vs sham) upregulated, and STAT1-F77A (MI vs sham) downregulated. 
Additionally, we used volcano plots to represent DESeq2 differential expression analysis results in animals who underwent ligation of the LAD vs sham operation in wild-type and STAT1-F77A mice, with $\mathrm{y}$-axis as $-\log _{10}$-adjusted $p$-value and the $\mathrm{x}$-axis as the $\log _{2}$-fold change (Figure 11 and Figure 12). In each plot, every point represents an individual transcript. The vertical lines represent a threshold of 1-fold change, either upregulated (right side) or downregulated (left side), while the horizontal lines represent an adjusted $p$-value $<0.05$. Colored points correspond to the uniquely differentially expressed genes in the following groups: 111 genes in wild-type MI vs sham upregulated (dark-turquoise), 83 genes in wild-type MI vs sham downregulated (light-turquoise), 909 genes in F77A MI vs sham upregulated (dark-orange), 1428 genes in F77A MI vs sham downregulated (light-orange), which corresponds to Figure 10 as well.
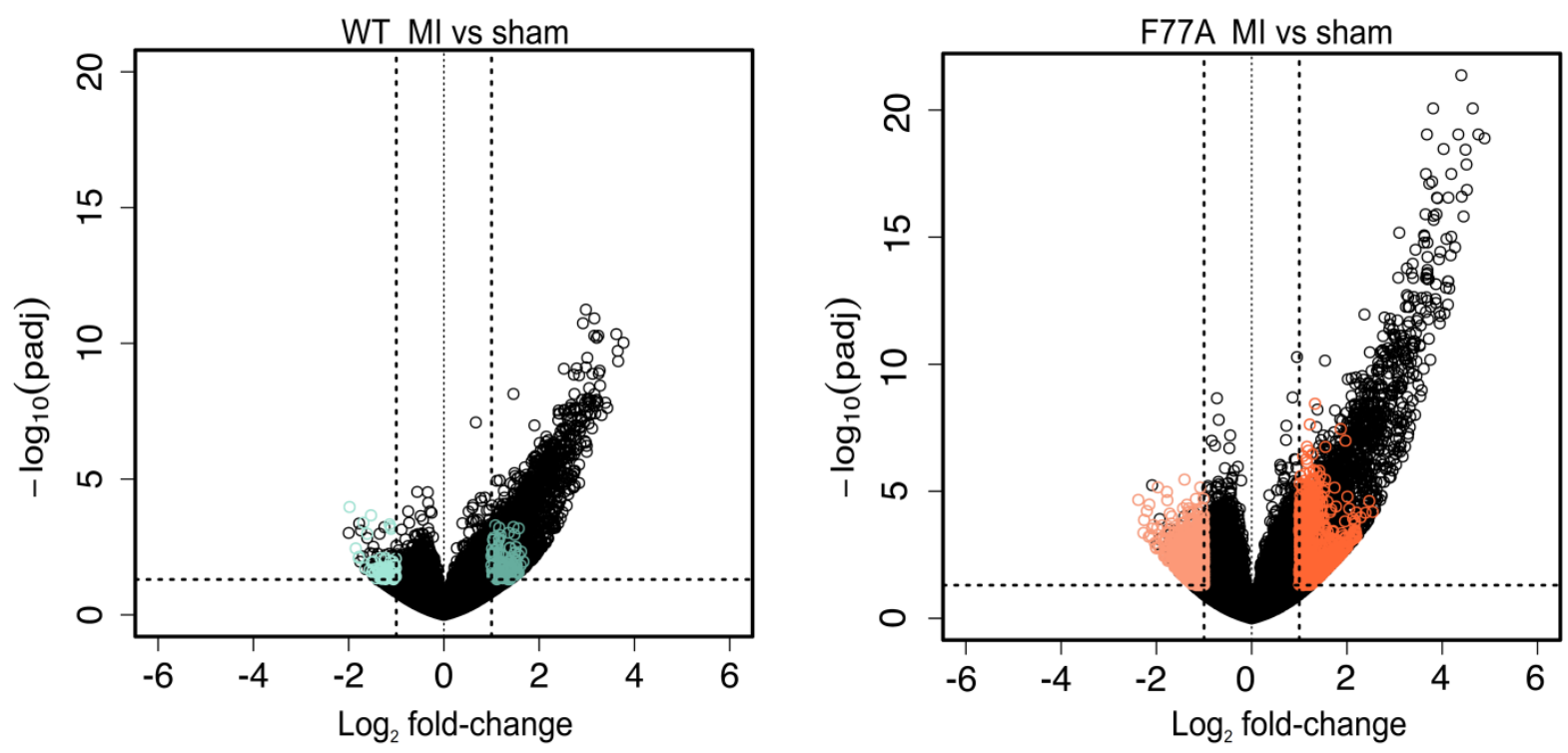

Figure 11: Volcano plot representation of differentially expressed genes in wild-type and STAT1-F77A mice post-myocardal infarction vs sham operated. Colored points correspond to uniquely upregulated or downregulated genes in the following pairwise comparisons: wild-type (MI vs sham) upregulated, wild-type (MI vs sham) downregulated, STAT1-F77A (MI vs sham) upregulated, and STAT1-F77A (MI vs sham) downregulated. 
A)

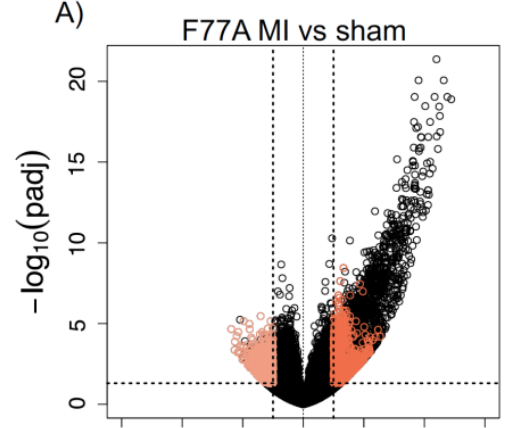

$\log _{2}$ fold-change

B)

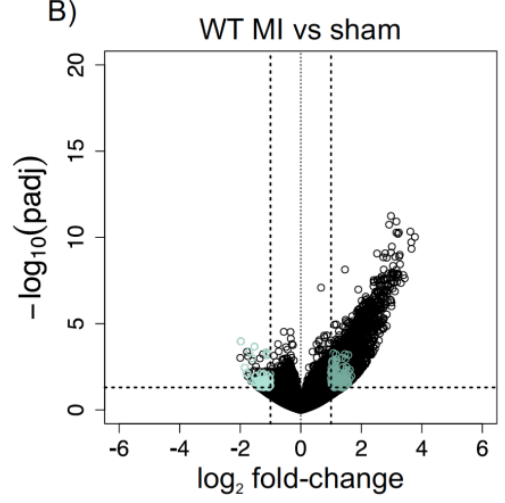

C)

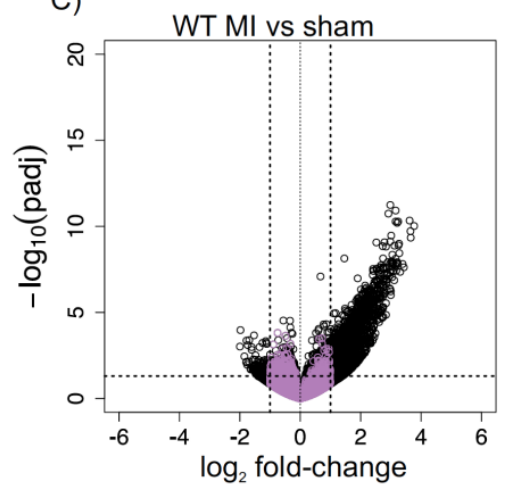

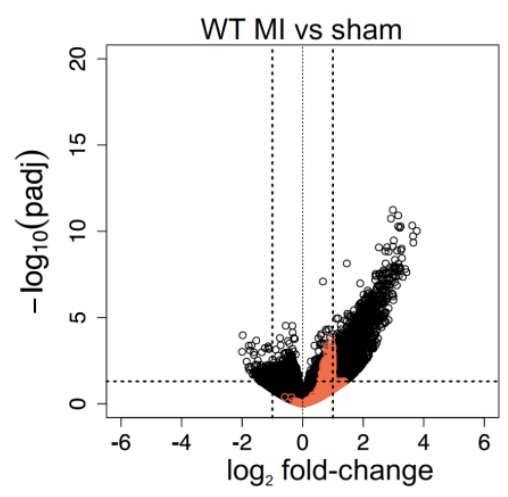
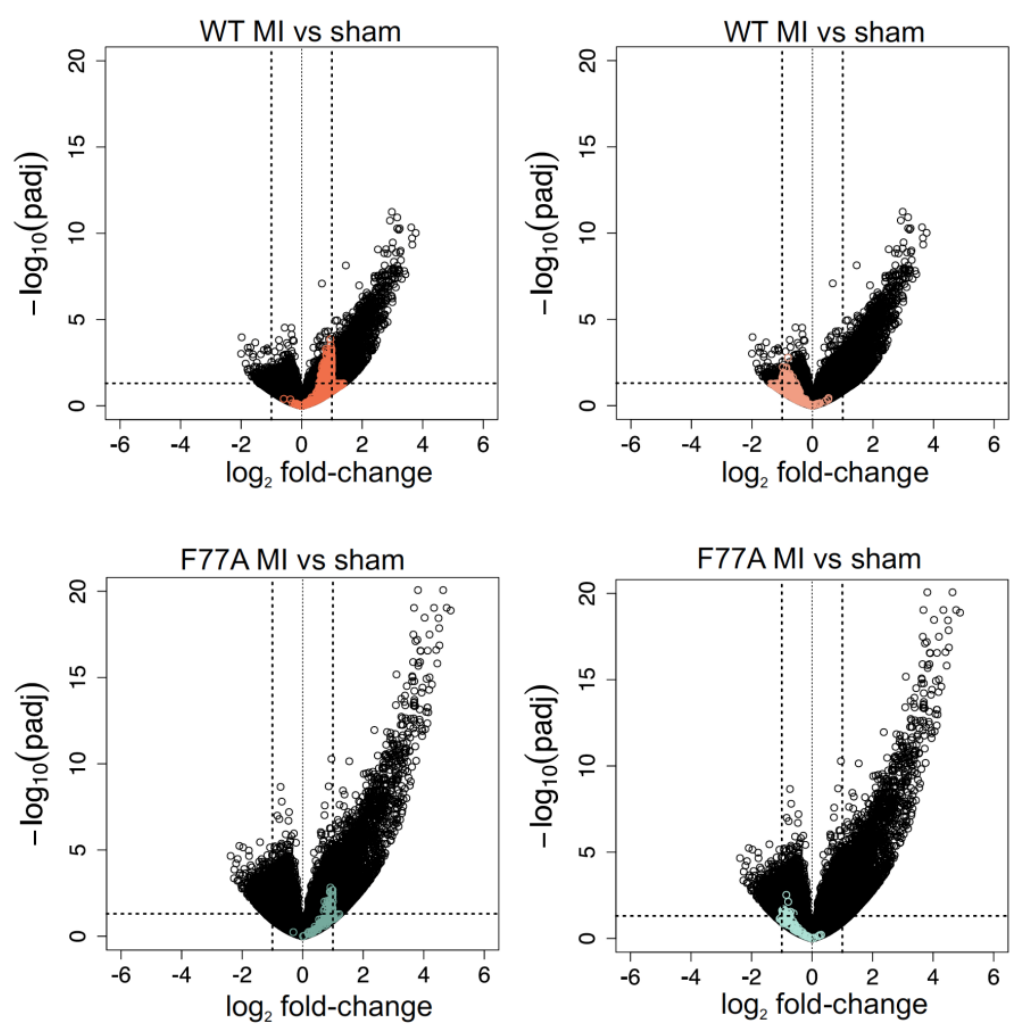

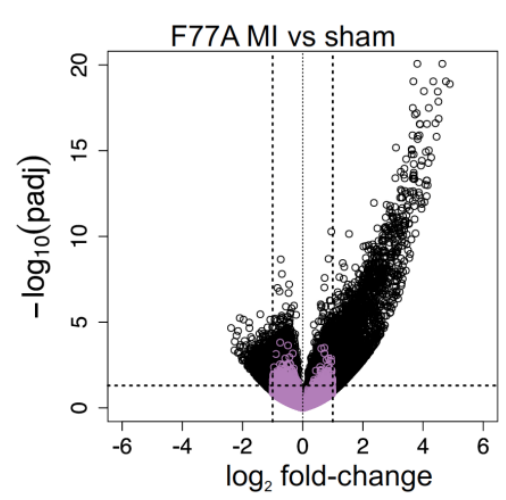

Figure 12: Volcano plot representation of uniquely differentially expressed genes in wild-type and STAT1-F77A (MI vs sham operated). (A) STAT1-F77A MI vs sham comparison. Colored points correspond to uniquely upregulated or downregulated genes in the STAT1-F77A MI vs sham comparison. The same genes are highlighted in the wild-type MI vs sham plots separately. (B) Wildtype MI vs sham. Colored points correspond to uniquely upregulated or downregulated genes in the wild-type MI vs sham comparison and the same genes are highlighted in the STAT1-F77A MI vs sham plots separately. (C) Colored points correspond to non-differentially expressed genes in the wild-type MI vs sham comparison and the same is highlighted in the F77A MI vs sham plot. 
Tables 25 and 26 illustrate the top 20 uniquely upregulated or downregulated differentially expressed genes in wild-type (LAD ligation vs sham) comparison, respectively.

Table 25: Top 20 uniquely upregulated genes in wild-type following LAD ligation

\begin{tabular}{lllll}
\hline Gene & $\log _{2}$ fold change & padj & $\log _{10}$ padj & baseMean \\
\hline Olfm 2 & 1.67 & $1.10 \mathrm{e}-02$ & $1.96 \mathrm{e}+00$ & 7.21 \\
\hline Unc13c & 1.62 & $4.75 \mathrm{e}-03$ & $2.32 \mathrm{e}+00$ & 18.22 \\
\hline Gp5 & 1.61 & $1.50 \mathrm{e}-02$ & $1.82 \mathrm{e}+00$ & 17.47 \\
\hline Cacnale & 1.57 & $6.56 \mathrm{e}-04$ & $3.18 \mathrm{e}+00$ & 18.02 \\
\hline Mospd4 & 1.57 & $2.77 \mathrm{e}-02$ & $1.56 \mathrm{e}+00$ & 4.27 \\
\hline Jakmip1 & 1.57 & $6.29 \mathrm{e}-03$ & $2.20 \mathrm{e}+00$ & 15.90 \\
\hline I700016P03Rik & 1.56 & $2.92 \mathrm{e}-02$ & $1.53 \mathrm{e}+00$ & 7.58 \\
\hline Snord58b & 1.54 & $2.51 \mathrm{e}-02$ & $1.60 \mathrm{e}+00$ & 5.60 \\
\hline Depdc1b & 1.53 & $1.47 \mathrm{e}-02$ & $1.83 \mathrm{e}+00$ & 6.53 \\
\hline Ighg2b & 1.53 & $3.14 \mathrm{e}-02$ & $1.50 \mathrm{e}+00$ & 3.81 \\
\hline Crhbp & 1.52 & $3.23 \mathrm{e}-02$ & $1.49 \mathrm{e}+00$ & 3.59 \\
\hline Col2a1 & 1.52 & $1.90 \mathrm{e}-02$ & $1.72 \mathrm{e}+00$ & 6.18 \\
\hline Lrtm2 & 1.52 & $1.00 \mathrm{e}-02$ & $2.00 \mathrm{e}+00$ & 13.59 \\
\hline Gm10288 & 1.51 & $2.55 \mathrm{e}-02$ & $1.59 \mathrm{e}+00$ & 9.14 \\
\hline Igha & 1.51 & $3.18 \mathrm{e}-03$ & $2.50 \mathrm{e}+00$ & 46.31 \\
\hline Spic & 1.50 & $8.90 \mathrm{e}-03$ & $2.05 \mathrm{e}+00$ & 11.16 \\
\hline Igsf11 & 1.50 & $4.84 \mathrm{e}-03$ & $2.32 \mathrm{e}+00$ & 44.37 \\
\hline Gm17619 & 1.50 & $3.40 \mathrm{e}-02$ & $1.47 \mathrm{e}+00$ & 4.77 \\
\hline Gm23301 & 1.48 & $5.33 \mathrm{e}-03$ & $2.27 \mathrm{e}+00$ & 18.45 \\
\hline Cebpd & 1.48 & $1.11 \mathrm{e}-03$ & $2.95 \mathrm{e}+00$ & 1707.34 \\
\hline
\end{tabular}

Table 26: Top 20 uniquely downregulated genes in wild-type following LAD ligation

\begin{tabular}{lllll}
\hline Gene (wild-type unique down) & $\log _{2}$ fold change & padj & $\log _{10}$ padj & baseMean \\
\hline Slitrk6 & -1.01 & $3.86 \mathrm{e}-02$ & $1.41 \mathrm{e}+00$ & 50.93 \\
\hline Dact2 & -1.01 & $3.85 \mathrm{e}-02$ & $1.41 \mathrm{e}+00$ & 51.37 \\
\hline Adamts17 & -1.01 & $8.55 \mathrm{e}-03$ & $2.07 \mathrm{e}+00$ & 44.52 \\
\hline Ccdc173 & -1.01 & $1.92 \mathrm{e}-02$ & $1.72 \mathrm{e}+00$ & 38.83 \\
\hline Ripk4 & -1.02 & $3.48 \mathrm{e}-02$ & $1.46 \mathrm{e}+00$ & 17.04 \\
\hline Gm17080 & -1.03 & $2.89 \mathrm{e}-02$ & $1.54 \mathrm{e}+00$ & 33.97 \\
\hline Gm15853 & -1.03 & $1.45 \mathrm{e}-02$ & $1.84 \mathrm{e}+00$ & 30.76 \\
\hline E330037G11Rik & -1.03 & $2.69 \mathrm{e}-02$ & $1.57 \mathrm{e}+00$ & 19.74 \\
\hline Fgf14 & -1.05 & $3.86 \mathrm{e}-02$ & $1.41 \mathrm{e}+00$ & 70.66 \\
\hline Pdzrn4 & -1.05 & $2.62 \mathrm{e}-02$ & $1.58 \mathrm{e}+00$ & 133.42 \\
\hline Rassf9 & -1.05 & $4.06 \mathrm{e}-02$ & $1.39 \mathrm{e}+00$ & 626.03 \\
\hline Ict1os & -1.06 & $2.60 \mathrm{e}-02$ & $1.59 \mathrm{e}+00$ & 17.01 \\
\hline Gm28523 & -1.06 & $1.60 \mathrm{e}-02$ & $1.80 \mathrm{e}+00$ & 56.84 \\
\hline Gm43075 & $4.64 \mathrm{e}-02$ & $1.33 \mathrm{e}+00$ & 12.46 \\
\hline Gm45809 & -1.06 & $4.61 \mathrm{e}-02$ & $1.34 \mathrm{e}+00$ & 10.64 \\
\hline Gm6581 & -1.06 & $4.93 \mathrm{e}-02$ & $1.31 \mathrm{e}+00$ & 11.72 \\
\hline BC065397 & -1.06 & $1.10 \mathrm{e}-02$ & $1.96 \mathrm{e}+00$ & 92.85 \\
\hline 5033417F24Rik & -1.07 & $1.92 \mathrm{e}-02$ & $1.72 \mathrm{e}+00$ & 23.30 \\
\hline Syt15 & -1.07 & $2.79 \mathrm{e}-02$ & $1.55 \mathrm{e}+00$ & 43.60 \\
\hline Gm20518 & -1.08 & $1.91 \mathrm{e}-02$ & $1.72 \mathrm{e}+00$ & 18.63 \\
\hline
\end{tabular}


Tables 27 and 28 illustrate the top 20 uniquely upregulated or downregulated differentially expressed genes in STAT1-F77A (LAD ligation vs sham) comparison, respectively.

Table 27: Top 20 uniquely upregulated genes in STAT1-F77A following LAD ligation

\begin{tabular}{lllll}
\hline Gene (F77A unique UP) & $\log _{2}$ fold change & padj & $\log _{10}$ padj & baseMean \\
\hline Tnfsf15 & 2.54 & $6.45 \mathrm{e}-05$ & $4.19 \mathrm{e}+00$ & 3.93 \\
\hline Trdc & 2.47 & $2.43 \mathrm{e}-05$ & $4.61 \mathrm{e}+00$ & 10.88 \\
\hline Gm10800 & 2.39 & $6.96 \mathrm{e}-05$ & $4.16 \mathrm{e}+00$ & 140.52 \\
\hline Gm19610 & 2.29 & $1.06 \mathrm{e}-04$ & $3.97 \mathrm{e}+00$ & 6.86 \\
\hline Muc2 & 2.20 & $6.83 \mathrm{e}-05$ & $4.17 \mathrm{e}+00$ & 26.84 \\
\hline Khdc3 & 2.20 & $5.70 \mathrm{e}-04$ & $3.24 \mathrm{e}+00$ & 4.04 \\
\hline Gm40304 & 2.20 & $5.74 \mathrm{e}-04$ & $3.24 \mathrm{e}+00$ & 4.81 \\
\hline Gm15922 & 2.19 & $4.36 \mathrm{e}-04$ & $3.36 \mathrm{e}+00$ & 10.23 \\
\hline Gm38575 & 2.19 & $6.16 \mathrm{e}-04$ & $3.21 \mathrm{e}+00$ & 3.21 \\
\hline Gm10801 & 2.18 & $4.00 \mathrm{e}-04$ & $3.40 \mathrm{e}+00$ & 16.00 \\
\hline Gm16853 & 2.18 & $3.40 \mathrm{e}-04$ & $3.47 \mathrm{e}+00$ & 4.66 \\
\hline Slc22a20 & 2.17 & $6.31 \mathrm{e}-04$ & $3.20 \mathrm{e}+00$ & 3.17 \\
\hline 5830428 M24Rik & 2.17 & $1.46 \mathrm{e}-04$ & $3.84 \mathrm{e}+00$ & 19.90 \\
\hline Chrnb3 & 2.16 & $7.11 \mathrm{e}-04$ & $3.15 \mathrm{e}+00$ & 5.36 \\
\hline Gpr15 & 2.16 & $1.95 \mathrm{e}-04$ & $3.71 \mathrm{e}+00$ & 7.39 \\
\hline Irgc1 & 2.15 & $5.52 \mathrm{e}-04$ & $3.26 \mathrm{e}+00$ & 4.32 \\
\hline Gm10717 & $6.31 \mathrm{e}-04$ & $3.20 \mathrm{e}+00$ & 4.74 \\
\hline Gm13748 & 3.15 & $1.18 \mathrm{e}-04$ & $3.40 \mathrm{e}+00$ & 8.37 \\
\hline E230014E18Rik & 2.14 & $5.47 \mathrm{e}-04$ & $3.26 \mathrm{e}+00$ & 16.59 \\
\hline Gm42141 & 2.13 & & & 9.37 \\
\hline
\end{tabular}

Table 28: Top 20 uniquely downregulated genes in STAT1-F77A following LAD ligation

\begin{tabular}{lllll}
\hline Gene & $\log _{2}$ fold change & padj & $\log _{10}$ padj & baseMean \\
\hline Cd36 & -1.00 & $4.51 \mathrm{e}-03$ & $2.35 \mathrm{e}+00$ & 72449.49 \\
\hline Rmnd1 & -1.00 & $6.19 \mathrm{e}-03$ & $2.21 \mathrm{e}+00$ & 851.78 \\
\hline Gnb3 & -1.00 & $1.24 \mathrm{e}-02$ & $1.91 \mathrm{e}+00$ & 168.96 \\
\hline Dusp23 & -1.00 & $8.89 \mathrm{e}-05$ & $4.05 \mathrm{e}+00$ & 454.24 \\
\hline Rgs5 & -1.00 & $6.41 \mathrm{e}-03$ & $2.19 \mathrm{e}+00$ & 22728.88 \\
\hline Nipal3 & -1.00 & $5.40 \mathrm{e}-03$ & $2.27 \mathrm{e}+00$ & 1366.59 \\
\hline Zfp606 & -1.00 & $3.36 \mathrm{e}-03$ & $2.47 \mathrm{e}+00$ & 613.67 \\
\hline Myom2 & -1.00 & $2.38 \mathrm{e}-03$ & $2.62 \mathrm{e}+00$ & 69623.68 \\
\hline Macrod1 & -1.00 & $2.76 \mathrm{e}-04$ & $3.56 \mathrm{e}+00$ & 5880.34 \\
\hline D630003M21Rik & -1.00 & $2.89 \mathrm{e}-02$ & $1.54 \mathrm{e}+00$ & 465.21 \\
\hline Uqcrfs1 & -1.00 & $8.79 \mathrm{e}-04$ & $3.06 \mathrm{e}+00$ & 28587.72 \\
\hline Fam13c & -1.00 & $1.72 \mathrm{e}-02$ & $1.76 \mathrm{e}+00$ & 291.44 \\
\hline Cxxc4 & -1.00 & $2.06 \mathrm{e}-02$ & $1.69 \mathrm{e}+00$ & 183.16 \\
\hline Kcnb1 & -1.00 & $5.70 \mathrm{e}-03$ & $2.24 \mathrm{e}+00$ & 2996.43 \\
\hline Gm9947 & -1.00 & $4.03 \mathrm{e}-02$ & $1.39 \mathrm{e}+00$ & 10.70 \\
\hline Camk2b & -1.00 & $2.07 \mathrm{e}-03$ & $2.68 \mathrm{e}+00$ & 573.18 \\
\hline Cd59b & -1.00 & $6.76 \mathrm{e}-03$ & $2.17 \mathrm{e}+00$ & 54.54 \\
\hline Gm10604 & -1.00 & $4.75 \mathrm{e}-02$ & $1.32 \mathrm{e}+00$ & 12.84 \\
\hline Gm13657 & -1.00 & $4.62 \mathrm{e}-02$ & $1.34 \mathrm{e}+00$ & 17.66 \\
\hline Spa17 & -1.01 & $5.45 \mathrm{e}-03$ & $2.26 \mathrm{e}+00$ & 72.01 \\
\hline
\end{tabular}




\subsection{Gene set enrichment analysis identified common and unique pathways associated with myocardial infarction}

To gain biological insight into the physiological role of cooperative DNA binding of STAT1 transcription factor on the global transcriptional regulation in acute myocardial infarction, we aimed to characterize the transcriptome of normal and infarcted heart tissue on day 1 from both wild-type and STAT1-F77A mice following LAD ligation. The top enriched pathways in the myocardial tissue of wild-type and STAT1-F77A animals post-MI were identified. To explore shared functions among differentially expressed genes, a common approach is to integrate the biological information, such as Gene Ontology (GO) and Kyoto Encyclopedia of Genes and Genomes (KEGG), for classifying major biological themes of gene sets. In these analyses, we tested for coordinated differential expression over gene sets from KEGG pathways rather than changes of individual genes. Generally applicable gene-set enrichment analysis was performed using the GAGE package on the differentially expressed genes. The $\log _{2}$-fold changes for all the genes from the DEseq 2 differential expression analysis were used as an input for GAGE analysis. We assumed that perturbations over a specific pathway may propose mechanistic changes post-myocardial infarction. In addition, gene expression profiles that are genotype dependent can be identified. GAGE uses fold changes for each gene to calculate mean and standard deviation of fold changes for a specific gene set (pathway), as well as for the whole transcriptome as a background, generating $t$-test statistics and a $p$-value for each gene set including the background in the respective comparison. Finally, to integrate and visualize enriched pathways, the Pathview $\mathrm{R}$ package was used to render the data (Gentleman et al., 2004). KEGG pathways that were identified as significantly differentially regulated were visualized using PathView, where upregulated genes are displayed in red and downregulated genes are in green and an unregulated gene in grey. The pathway is considered to be generally upregulated if the majority of genes are colored in red. A higher enrichment mean corresponds to a shifting of gene set constituents towards either end of the ranked list representing strongly upregulated or downregulated pathways.

The differentially regulated pathways demonstrate that differentially expressed genes were mainly enriched in four main categories, which are (1) immune processes, (2) signal transduction, (3) cellular processes and (4) metabolism. The enrichment scores for both immune- and extracellular matrix remodeling-related pathways were significantly higher, while metabolism-related pathways had higher negative enrichment scores in both wild-type and STAT1-F77A after myocardial infarction. The top gene set enrichment analysis (GSEA)identified pathways for wild-type (LAD ligation vs sham) comparison with q-value $<0.1$ are 
shown in Table 29 and Figure 13, and similarly for STAT1-F77A (LAD ligation vs sham) comparison in Table 30 and Figure 14, while Figure 15 lists both genotypes.

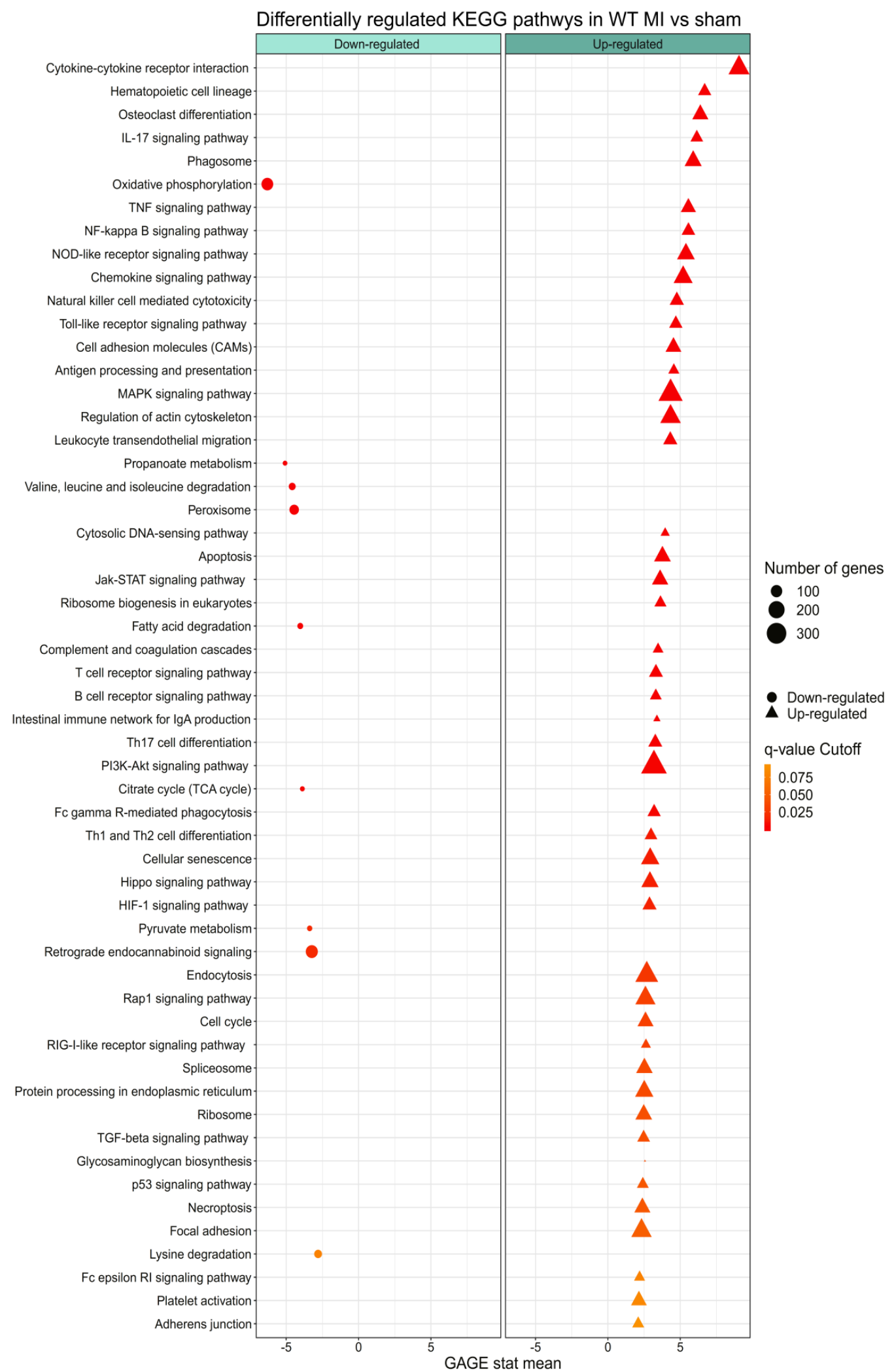

Figure 13: List of KEGG differentially regulated pathways in wild-type mice post-MI. Table 29: List of KEGG differentially regulated pathways following LAD ligation in wild-type mice. 


\begin{tabular}{|c|c|c|c|c|c|c|}
\hline & KEGG ID & KEGG pathway (wild-type MI vs sham) & mean & p value & q value & size \\
\hline \multirow{21}{*}{ 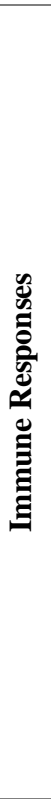 } & $\mathrm{mmu} 04060$ & Cytokine-cytokine receptor interaction & 9.1 & $4.66 \mathrm{e}-18$ & $1.03 \mathrm{e}-15$ & 204 \\
\hline & mmu04062 & Chemokine signalling pathway & 5.2 & $1.70 \mathrm{e}-07$ & $4.16 \mathrm{e}-06$ & 167 \\
\hline & $\mathrm{mmu} 04514$ & Cell adhesion molecules (CAMs) & 4.5 & $4.80 \mathrm{e}-06$ & $8.80 \mathrm{e}-05$ & 117 \\
\hline & $\mathrm{mmu} 04810$ & Regulation of actin cytoskeleton & 4.3 & $9.56 \mathrm{e}-06$ & $1.40 \mathrm{e}-04$ & 195 \\
\hline & mmu04670 & Leukocyte transendothelial migration & 4.3 & $1.32 \mathrm{e}-05$ & $1.82 \mathrm{e}-04$ & 95 \\
\hline & $\mathrm{mmu} 04510$ & Focal adhesion & 2.3 & $1.03 \mathrm{e}-02$ & $5.29 \mathrm{e}-02$ & 192 \\
\hline & mmu04611 & Platelet activation & 2.1 & $1.66 \mathrm{e}-02$ & $8.10 \mathrm{e}-02$ & 116 \\
\hline & $\mathrm{mmu} 04520$ & Adherens junction & 2.1 & $1.91 \mathrm{e}-02$ & $9.14 \mathrm{e}-02$ & 70 \\
\hline & $\mathrm{mmu} 04620$ & Toll-like receptor signalling pathway & 4.7 & $2.93 \mathrm{e}-06$ & $5.86 \mathrm{e}-05$ & 85 \\
\hline & mmu04666 & Fc $\gamma R$-mediated phagocytosis & 3.2 & $8.39 \mathrm{e}-04$ & $6.84 \mathrm{e}-03$ & 85 \\
\hline & $\mathrm{mmu} 04640$ & Hematopoietic cell lineage & 6.7 & $2.00 \mathrm{e}-10$ & $2.20 \mathrm{e}-08$ & 84 \\
\hline & mmu04659 & Th17 cell differentiation & 3.3 & $6.32 \mathrm{e}-04$ & $5.56 \mathrm{e}-03$ & 93 \\
\hline & $\mathrm{mmu} 04657$ & IL-17 signalling pathway & 6.1 & $4.73 \mathrm{e}-09$ & $2.60 \mathrm{e}-07$ & 78 \\
\hline & mmu04660 & T cell receptor signalling pathway & 3.3 & $5.44 \mathrm{e}-04$ & $5.44 \mathrm{e}-03$ & 93 \\
\hline & $\mathrm{mmu} 04658$ & Th1 and Th2 cell differentiation & 3 & $1.69 \mathrm{e}-03$ & $1.32 \mathrm{e}-02$ & 79 \\
\hline & mmu04662 & B cell receptor signalling pathway & 3.3 & $5.96 \mathrm{e}-04$ & $5.56 \mathrm{e}-03$ & 69 \\
\hline & mmu04650 & Natural killer cell mediated cytotoxicity & 4.8 & $1.93 \mathrm{e}-06$ & $4.25 \mathrm{e}-05$ & 96 \\
\hline & mmu04672 & Intestinal immune network for $\operatorname{Ig} \mathrm{A}$ production & 3.4 & $6.20 \mathrm{e}-04$ & $5.56 \mathrm{e}-03$ & 34 \\
\hline & mmu04610 & Complement and coagulation cascades & 3.5 & $3.91 \mathrm{e}-04$ & $4.09 \mathrm{e}-03$ & 59 \\
\hline & mmu04664 & Fc epsilon RI signalling pathway & 2.2 & $1.54 \mathrm{e}-02$ & $7.69 \mathrm{e}-02$ & 61 \\
\hline & $\mathrm{mmu} 04612$ & Antigen processing and presentation & 4.6 & $6.35 \mathrm{e}-06$ & $1.07 \mathrm{e}-04$ & 62 \\
\hline \multirow{14}{*}{ 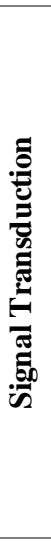 } & mmu04151 & PI3K-Akt signalling pathway & 3.2 & $7.66 \mathrm{e}-04$ & $6.48 \mathrm{e}-03$ & 306 \\
\hline & mmu04630 & Jak-STAT signalling pathway & 3.6 & $1.89 \mathrm{e}-04$ & $2.18 \mathrm{e}-03$ & 124 \\
\hline & $\mathrm{mmu} 04668$ & TNF signalling pathway & 5.6 & $4.48 \mathrm{e}-08$ & $1.62 \mathrm{e}-06$ & 108 \\
\hline & mmu04064 & NF- $\kappa \mathrm{B}$ signalling pathway & 5.6 & $5.15 \mathrm{e}-08$ & $1.62 \mathrm{e}-06$ & 89 \\
\hline & mmu04621 & NOD-like receptor signalling pathway & 5.4 & $7.44 \mathrm{e}-08$ & $2.04 \mathrm{e}-06$ & 146 \\
\hline & mmu04010 & MAPK signalling pathway & 4.3 & $8.88 \mathrm{e}-06$ & $1.40 \mathrm{e}-04$ & 272 \\
\hline & $\mathrm{mmu} 04380$ & Osteoclast differentiation & 6.4 & $4.91 \mathrm{e}-10$ & $3.60 \mathrm{e}-08$ & 122 \\
\hline & mmu04066 & HIF-1 signalling pathway & 2.9 & $2.25 \mathrm{e}-03$ & $1.60 \mathrm{e}-02$ & 95 \\
\hline & mmu04350 & TGF- $\beta$ signalling pathway & 2.7 & $3.95 \mathrm{e}-03$ & $2.63 e-02$ & 73 \\
\hline & mmu04015 & Rap1 signalling pathway & 2.6 & $4.93 \mathrm{e}-03$ & $3.03 e-02$ & 188 \\
\hline & mmu04622 & RIG-I-like receptor signalling pathway & 2.6 & $4.95 \mathrm{e}-03$ & $3.03 \mathrm{e}-02$ & 52 \\
\hline & mmu04390 & Hippo signalling pathway & 2.9 & $1.98 \mathrm{e}-03$ & $1.45 \mathrm{e}-02$ & 138 \\
\hline & $\mathrm{mmu} 04115$ & p53 signalling pathway & 2.4 & $8.59 \mathrm{e}-03$ & $4.61 \mathrm{e}-02$ & 69 \\
\hline & $\mathrm{mmu} 04623$ & Cytosolic DNA-sensing pathway & 4 & $7.89 \mathrm{e}-05$ & $1.02 \mathrm{e}-03$ & 46 \\
\hline \multirow{12}{*}{ 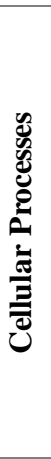 } & mmu04144 & Endocytosis & 2.7 & $3.74 \mathrm{e}-03$ & $2.57 \mathrm{e}-02$ & 240 \\
\hline & $\mathrm{mmu} 04110$ & Cell cycle & 2.6 & $4.95 \mathrm{e}-03$ & $3.03 e-02$ & 119 \\
\hline & mmu04218 & Cellular senescence & 2.9 & $1.87 \mathrm{e}-03$ & $1.42 \mathrm{e}-02$ & 155 \\
\hline & mmu04210 & Apoptosis & 3.8 & $1.02 \mathrm{e}-04$ & $1.24 \mathrm{e}-03$ & 131 \\
\hline & mmu04217 & Necroptosis & 2.4 & $8.96 \mathrm{e}-03$ & $4.69 \mathrm{e}-02$ & 121 \\
\hline & mmu03010 & Ribosome & 2.5 & $6.99 \mathrm{e}-03$ & $3.94 \mathrm{e}-02$ & 130 \\
\hline & $\mathrm{mmu} 04145$ & Phagosome & 5.9 & $5.96 \mathrm{e}-09$ & $2.62 \mathrm{e}-07$ & 137 \\
\hline & $\mathrm{mmu} 04146$ & Peroxisome & -4.5 & $8.56 \mathrm{e}-06$ & $4.71 \mathrm{e}-04$ & 78 \\
\hline & $\mathrm{mmu} 03040$ & Spliceosome & 2.5 & $6.16 \mathrm{e}-03$ & $3.63 e-02$ & 127 \\
\hline & $\mathrm{mmu} 04141$ & Protein processing in endoplasmic reticulum & 2.5 & $6.26 \mathrm{e}-03$ & $3.63 e-02$ & 155 \\
\hline & mmu04723 & Retrograde endocannabinoid signalling & -3.2 & $6.97 \mathrm{e}-04$ & $1.92 \mathrm{e}-02$ & 122 \\
\hline & mmu03008 & Ribosome biogenesis in eukaryotes & 3.6 & $2.05 \mathrm{e}-04$ & $2.26 \mathrm{e}-03$ & 72 \\
\hline \multirow{10}{*}{ 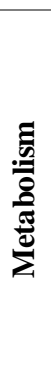 } & mmu00190 & Oxidative phosphorylation & -6.3 & $1.28 \mathrm{e}-09$ & $2.82 \mathrm{e}-07$ & 116 \\
\hline & mmu00020 & Citrate cycle (TCA cycle) & -3.9 & $1.84 \mathrm{e}-04$ & $6.74 \mathrm{e}-03$ & 30 \\
\hline & $\mathrm{mmu} 01212$ & Fatty acid metabolism & -2.8 & $3.51 \mathrm{e}-03$ & $7.72 \mathrm{e}-02$ & 49 \\
\hline & $\mathrm{mmu} 00071$ & Fatty acid degradation & -4 & $7.69 \mathrm{e}-05$ & $3.39 \mathrm{e}-03$ & 37 \\
\hline & $\mathrm{mmu} 00640$ & Propanoate metabolism & -5.1 & $4.08 \mathrm{e}-06$ & $4.49 \mathrm{e}-04$ & 29 \\
\hline & mmu00310 & Lysine degradation & -2.8 & $3.24 \mathrm{e}-03$ & $7.72 \mathrm{e}-02$ & 56 \\
\hline & mmu00380 & Tryptophan metabolism & -2.7 & $4.39 \mathrm{e}-03$ & $8.79 \mathrm{e}-02$ & 33 \\
\hline & mmu00620 & Pyruvate metabolism & -3.4 & $6.88 \mathrm{e}-04$ & $1.92 \mathrm{e}-02$ & 33 \\
\hline & $\mathrm{mmu} 00280$ & Valine, leucine and isoleucine degradation & -4.6 & $7.44 \mathrm{e}-06$ & $4.71 \mathrm{e}-04$ & 47 \\
\hline & mmu00532 & Glycosaminoglycan biosynthesis & 2.6 & $7.39 \mathrm{e}-03$ & $4.06 \mathrm{e}-02$ & 20 \\
\hline
\end{tabular}




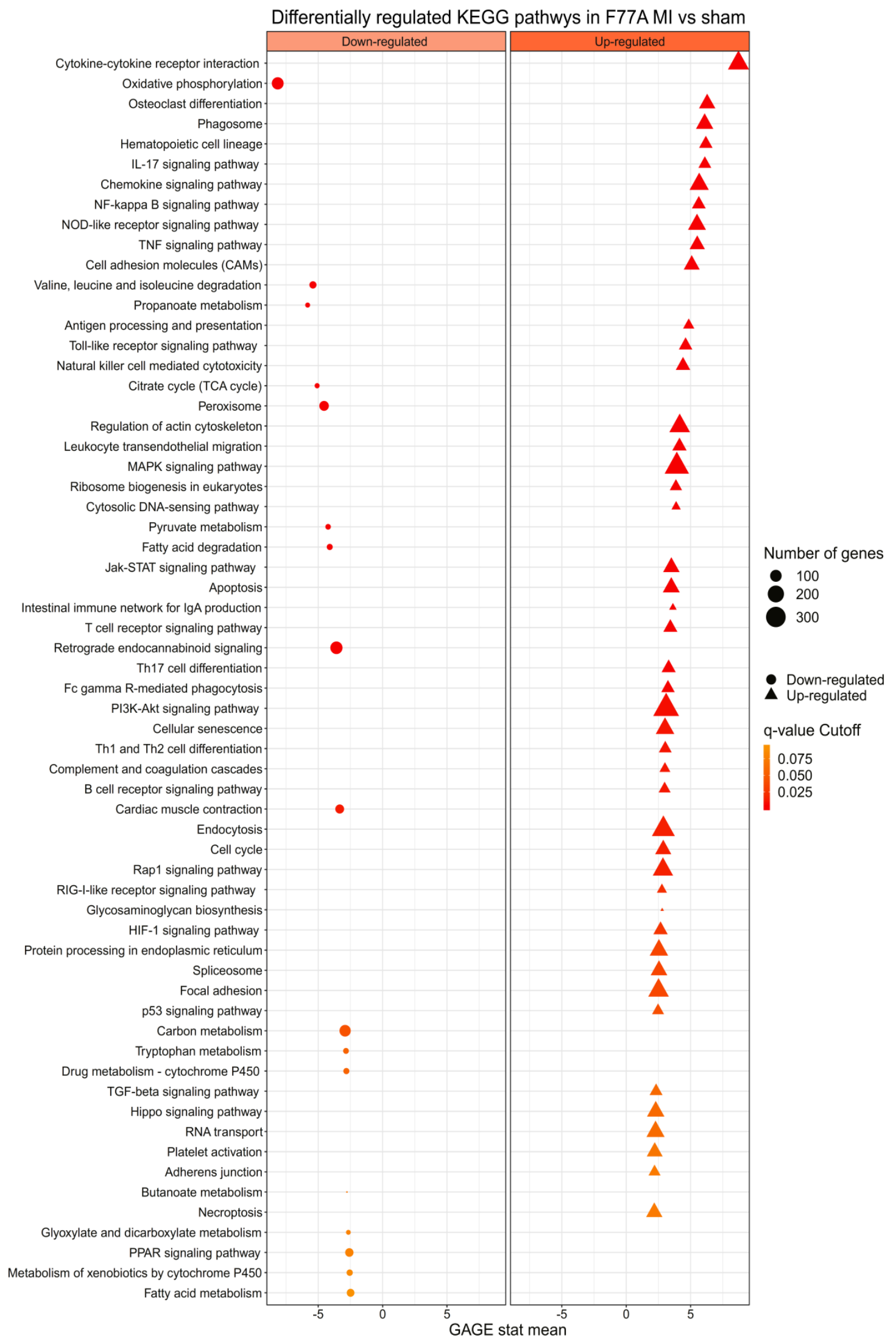

Figure 14: List of KEGG differentially regulated pathways following LAD ligation in transgenic mice expressing STAT1-F77A. 
Table 30: List of KEGG differentially regulated pathways following LAD ligation in STAT1 F77A mice

\begin{tabular}{|c|c|c|c|c|c|c|}
\hline & KEGG ID & KEGG pathway (STAT1-F77A MI vs sham) & mean & p value & q value & size \\
\hline \multirow{20}{*}{ 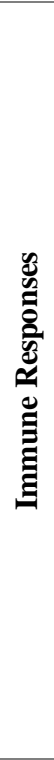 } & mmu04060 & Cytokine-cytokine receptor interaction & 8.7 & $6.39 \mathrm{e}-17$ & $1.41 \mathrm{e}-14$ & 204 \\
\hline & mmu04062 & Chemokine signalling pathway & 5.7 & $1.53 \mathrm{e}-08$ & $5.60 \mathrm{e}-07$ & 167 \\
\hline & $\mathrm{mmu04514}$ & Cell adhesion molecules (CAMs) & 5.1 & $3.87 \mathrm{e}-07$ & $8.51 \mathrm{e}-06$ & 117 \\
\hline & mmu04810 & Regulation of actin cytoskeleton & 4.2 & $2.03 \mathrm{e}-05$ & $3.19 \mathrm{e}-04$ & 195 \\
\hline & mmu04670 & Leukocyte transendothelial migration & 4.1 & $2.71 \mathrm{e}-05$ & $3.97 \mathrm{e}-04$ & 95 \\
\hline & mmu04510 & Focal adhesion & 2.5 & $6.26 \mathrm{e}-03$ & $3.53 \mathrm{e}-02$ & 192 \\
\hline & mmu04611 & Platelet activation & 2.2 & $1.40 \mathrm{e}-02$ & $7.18 \mathrm{e}-02$ & 116 \\
\hline & mmu04520 & Adherens junction & 2.2 & $1.48 \mathrm{e}-02$ & $7.40 \mathrm{e}-02$ & 70 \\
\hline & mmu04620 & Toll-like receptor signalling pathway & 4.6 & $4.14 \mathrm{e}-06$ & $7.60 \mathrm{e}-05$ & 85 \\
\hline & mmu04666 & Fc $\gamma$ R-mediated phagocytosis & 3.2 & $7.22 \mathrm{e}-04$ & $6.62 \mathrm{e}-03$ & 85 \\
\hline & mmu04640 & Hematopoietic cell lineage & 6.2 & $2.89 \mathrm{e}-09$ & $1.59 \mathrm{e}-07$ & 84 \\
\hline & mmu04659 & Th17 cell differentiation & 3.3 & $5.96 \mathrm{e}-04$ & $5.70 \mathrm{e}-03$ & 93 \\
\hline & mmu04657 & IL-17 signalling pathway & 6.1 & 5.81e-09 & $2.56 \mathrm{e}-07$ & 78 \\
\hline & mmu04660 & T cell receptor signalling pathway & 3.4 & $3.77 \mathrm{e}-04$ & $3.77 \mathrm{e}-03$ & 93 \\
\hline & mmu04658 & Th1 and Th2 cell differentiation & 3 & $1.43 \mathrm{e}-03$ & $1.17 \mathrm{e}-02$ & 79 \\
\hline & mmu04662 & B cell receptor signalling pathway & 3 & $1.70 \mathrm{e}-03$ & $1.29 \mathrm{e}-02$ & 69 \\
\hline & mmu04650 & Natural killer cell mediated cytotoxicity & 4.4 & $8.80 \mathrm{e}-06$ & $1.49 \mathrm{e}-04$ & 96 \\
\hline & mmu04672 & Intestinal immune network for IgA production & 3.6 & $2.95 \mathrm{e}-04$ & $3.09 \mathrm{e}-03$ & 34 \\
\hline & mmu04610 & Complement and coagulation cascades & 3 & $1.69 \mathrm{e}-03$ & $1.29 \mathrm{e}-02$ & 59 \\
\hline & mmu04612 & Antigen processing and presentation & 4.9 & $1.83 \mathrm{e}-06$ & $3.66 \mathrm{e}-05$ & 62 \\
\hline \multirow{15}{*}{ 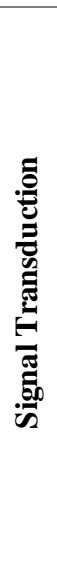 } & mmu04151 & PI3K-Akt signalling pathway & 3.1 & $1.02 \mathrm{e}-03$ & $8.96 \mathrm{e}-03$ & 306 \\
\hline & mmu04630 & Jak-STAT signalling pathway & 3.5 & $2.76 \mathrm{e}-04$ & $3.06 \mathrm{e}-03$ & 124 \\
\hline & $\mathrm{mmu} 04668$ & TNF signalling pathway & 5.5 & $5.72 \mathrm{e}-08$ & $1.40 \mathrm{e}-06$ & 108 \\
\hline & mmu04064 & $\mathrm{NF}-\kappa \mathrm{B}$ signalling pathway & 5.6 & $3.62 \mathrm{e}-08$ & $1.14 \mathrm{e}-06$ & 89 \\
\hline & $\mathrm{mmu} 04621$ & NOD-like receptor signalling pathway & 5.5 & $4.48 \mathrm{e}-08$ & $1.23 \mathrm{e}-06$ & 146 \\
\hline & mmu04010 & MAPK signalling pathway & 3.9 & $4.86 \mathrm{e}-05$ & $6.69 \mathrm{e}-04$ & 272 \\
\hline & $\mathrm{mmu} 04380$ & Osteoclast differentiation & 6.3 & $8.57 \mathrm{e}-10$ & $9.43 \mathrm{e}-08$ & 122 \\
\hline & mmu04066 & HIF-1 signalling pathway & 2.7 & $4.22 \mathrm{e}-03$ & $2.66 \mathrm{e}-02$ & 95 \\
\hline & mmu04350 & TGF- $\beta$ signalling pathway & 2.6 & $5.50 \mathrm{e}-03$ & $3.36 \mathrm{e}-02$ & 73 \\
\hline & mmu04015 & Rap1 signalling pathway & 2.8 & $2.32 \mathrm{e}-03$ & $1.60 \mathrm{e}-02$ & 188 \\
\hline & $\mathrm{mmu04622}$ & RIG-I-like receptor signalling pathway & 2.8 & $3.34 \mathrm{e}-03$ & $2.22 \mathrm{e}-02$ & 52 \\
\hline & mmu04390 & Hippo signalling pathway & 2.3 & $1.14 \mathrm{e}-02$ & $6.13 \mathrm{e}-02$ & 138 \\
\hline & mmu04115 & p53 signalling pathway & 2.5 & $7.40 \mathrm{e}-03$ & $4.07 \mathrm{e}-02$ & 69 \\
\hline & $\mathrm{mmu04623}$ & Cytosolic DNA-sensing pathway & 3.9 & $1.05 \mathrm{e}-04$ & $1.28 \mathrm{e}-03$ & 46 \\
\hline & mmu03013 & RNA transport & 2.3 & $1.19 \mathrm{e}-02$ & $6.25 \mathrm{e}-02$ & 150 \\
\hline \multirow{12}{*}{ 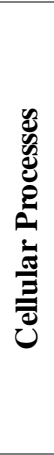 } & mmu04144 & Endocytosis & 2.9 & $2.05 \mathrm{e}-03$ & $1.50 \mathrm{e}-02$ & 240 \\
\hline & mmu04110 & Cell cycle & 2.9 & $2.24 \mathrm{e}-03$ & $1.59 \mathrm{e}-02$ & 119 \\
\hline & mmu04260 & Cardiac muscle contraction & -3.3 & $5.57 \mathrm{e}-04$ & $1.36 \mathrm{e}-02$ & 67 \\
\hline & mmu04218 & Cellular senescence & 3 & $1.40 \mathrm{e}-03$ & $1.17 \mathrm{e}-02$ & 155 \\
\hline & mmu04210 & Apoptosis & 3.5 & $2.79 \mathrm{e}-04$ & $3.06 \mathrm{e}-03$ & 131 \\
\hline & mmu04217 & Necroptosis & 2.2 & $1.52 \mathrm{e}-02$ & $7.44 \mathrm{e}-02$ & 121 \\
\hline & $\mathrm{mmu} 04145$ & Phagosome & 6.1 & $2.03 \mathrm{e}-09$ & $1.49 \mathrm{e}-07$ & 137 \\
\hline & mmu04146 & Peroxisome & -4.5 & $5.53 \mathrm{e}-06$ & $2.44 \mathrm{e}-04$ & 78 \\
\hline & mmu03040 & Spliceosome & 2.5 & $5.88 \mathrm{e}-03$ & $3.40 \mathrm{e}-02$ & 127 \\
\hline & mmu04141 & Protein processing in endoplasmic reticulum & 2.5 & $5.83 \mathrm{e}-03$ & $3.40 \mathrm{e}-02$ & 155 \\
\hline & mmu04723 & Retrograde endocannabinoid signalling & -3.6 & $2.02 \mathrm{e}-04$ & $5.56 \mathrm{e}-03$ & 122 \\
\hline & mmu03008 & Ribosome biogenes is in eukaryotes & 3.9 & $9.28 \mathrm{e}-05$ & $1.20 \mathrm{e}-03$ & 72 \\
\hline \multirow{14}{*}{ 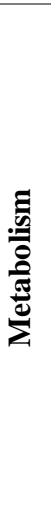 } & $\mathrm{mmu} 00190$ & Oxidative phosphorylation & -8.1 & $3.66 \mathrm{e}-14$ & $8.06 \mathrm{e}-12$ & 116 \\
\hline & mmu00020 & Citrate cycle (TCA cycle) & -5.1 & $3.38 \mathrm{e}-06$ & $1.86 \mathrm{e}-04$ & 30 \\
\hline & $\mathrm{mmu} 01212$ & Fatty acid metabolism & -2.8 & $3.56 \mathrm{e}-03$ & $6.02 \mathrm{e}-02$ & 49 \\
\hline & mmu00071 & Fatty acid degradation & -4.1 & $5.31 \mathrm{e}-05$ & $1.67 \mathrm{e}-03$ & 37 \\
\hline & mmu00640 & Propanoate metabolism & -5.8 & $3.59 \mathrm{e}-07$ & $2.63 \mathrm{e}-05$ & 29 \\
\hline & mmu00380 & Tryptophan metabolism & -3 & $2.01 \mathrm{e}-03$ & $4.01 \mathrm{e}-02$ & 33 \\
\hline & $\mathrm{mmu} 00620$ & Pyruvate metabolism & -4.2 & $4.22 \mathrm{e}-05$ & $1.55 \mathrm{e}-03$ & 33 \\
\hline & $\mathrm{mmu} 00280$ & Valine, leucine and isoleucine degradation & -5.4 & $2.58 \mathrm{e}-07$ & $2.63 \mathrm{e}-05$ & 47 \\
\hline & mmu00532 & Glycosaminoglycan biosynthesis & 2.8 & $4.19 \mathrm{e}-03$ & $2.66 \mathrm{e}-02$ & 20 \\
\hline & mmu00650 & Butanoate metabolism & -2.8 & $4.39 \mathrm{e}-03$ & $6.90 \mathrm{e}-02$ & 19 \\
\hline & mmu00630 & Glyoxylate and dicarboxylate metabolism & -2.7 & $5.15 \mathrm{e}-03$ & $7.55 \mathrm{e}-02$ & 28 \\
\hline & $\mathrm{mmu} 01200$ & Carbon metabolism & -2.9 & $1.98 \mathrm{e}-03$ & $4.01 \mathrm{e}-02$ & 106 \\
\hline & mmu00982 & Drug metabolism - cytochrome P450 & -2.8 & $3.12 \mathrm{e}-03$ & $5.72 \mathrm{e}-02$ & 37 \\
\hline & mmu03320 & PPAR signalling pathway & -2.6 & $5.49 \mathrm{e}-03$ & $7.55 \mathrm{e}-02$ & 61 \\
\hline
\end{tabular}


Differentially regulated KEGG pathwys in MI vs sham

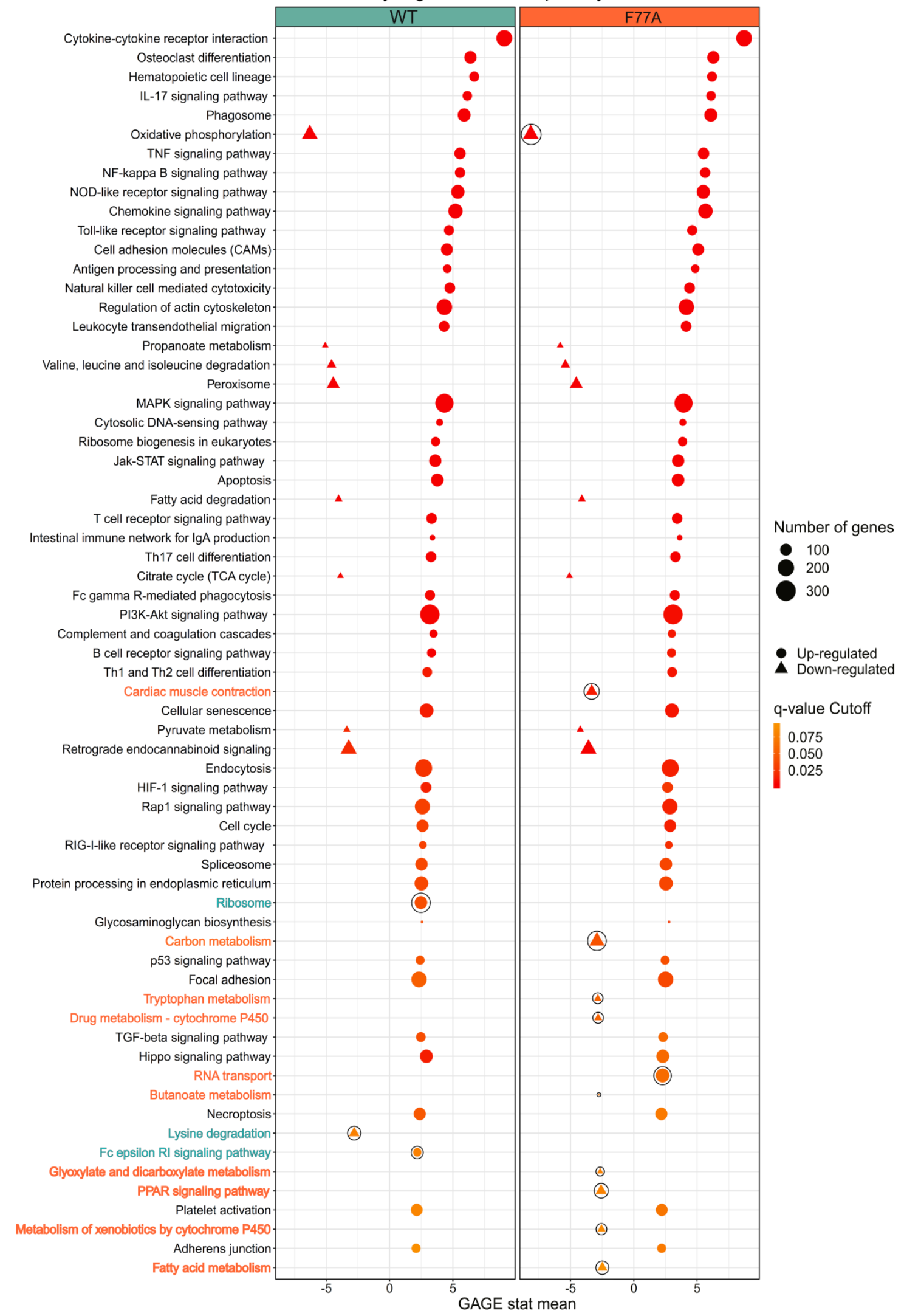

Figure 15: KEGG-regulated pathways following LAD ligation in the two STAT1 genotypes. 


\subsection{Identification of immune-related pathways differentially regulated after myocardial infarction}

In this study, KEGG pathways that were classified as immune processes significantly enriched post-myocardial infarction in both wild-type and STAT1-F77A mice were the following: cytokine-cytokine receptor interaction (Figure 17), chemokine signalling pathway (Figure 18), cell adhesion molecules (CAMs) (Figure 19), regulation of actin cytoskeleton (Figure 20), leukocyte transendothelial migration (Figure 21), focal adhesion, platelet activation, adherens junction, Toll-like receptor signalling pathway, FcyR-mediated phagocytosis, hematopoietic cell lineage, Th17 cell differentiation, IL-17 signalling pathway, T cell receptor signalling pathway, Th1 and Th2 cell differentiation, B cell receptor signalling pathway, natural killer cell-mediated cytotoxicity, intestinal immune network for $\operatorname{IgA}$ production, complement and coagulation cascades, Fc epsilon RI signalling pathway, and antigen processing and presentation.

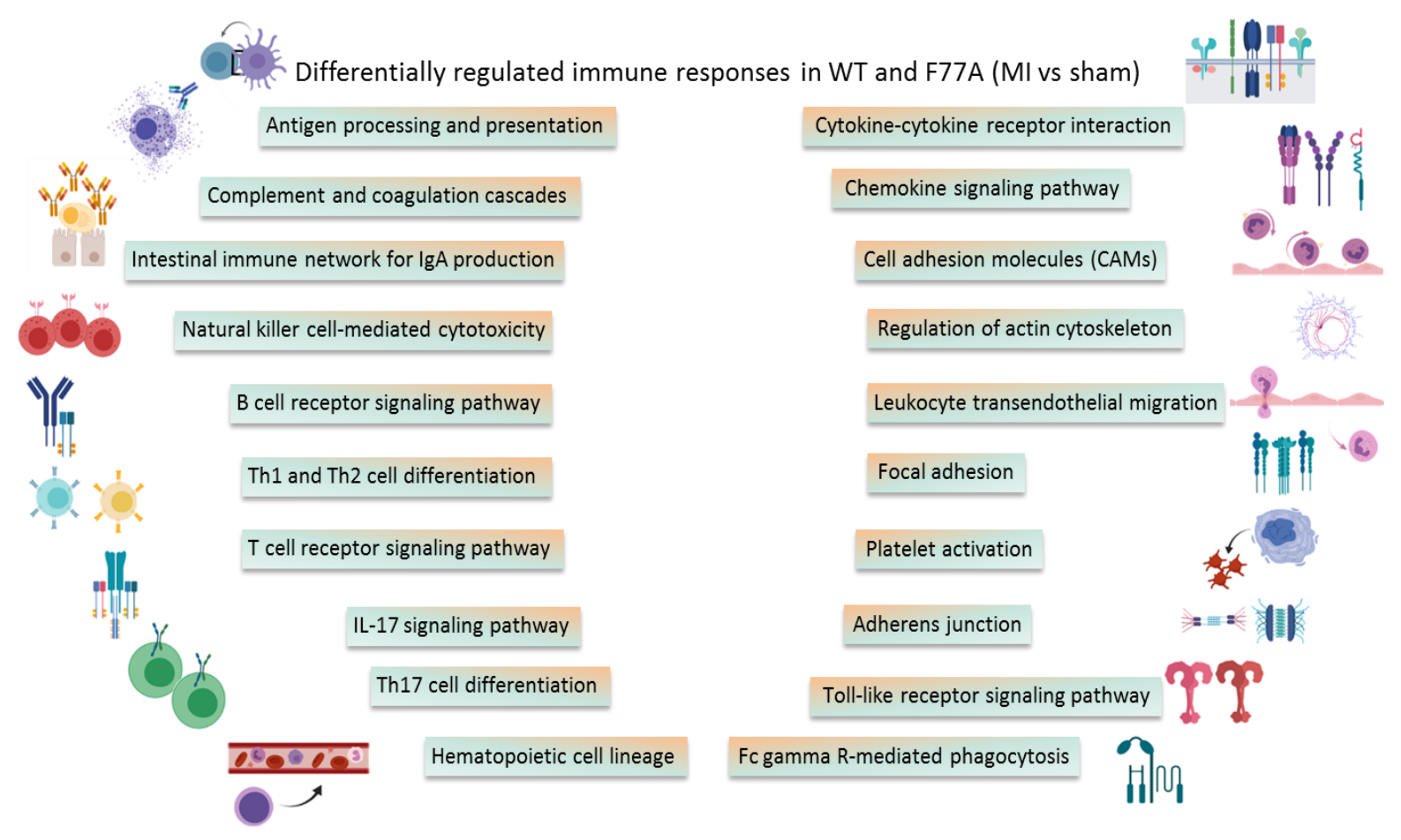

Figure 16: List of KEGG immune responses that were differentially regulated in both STAT1F77A and wild-type mice. 


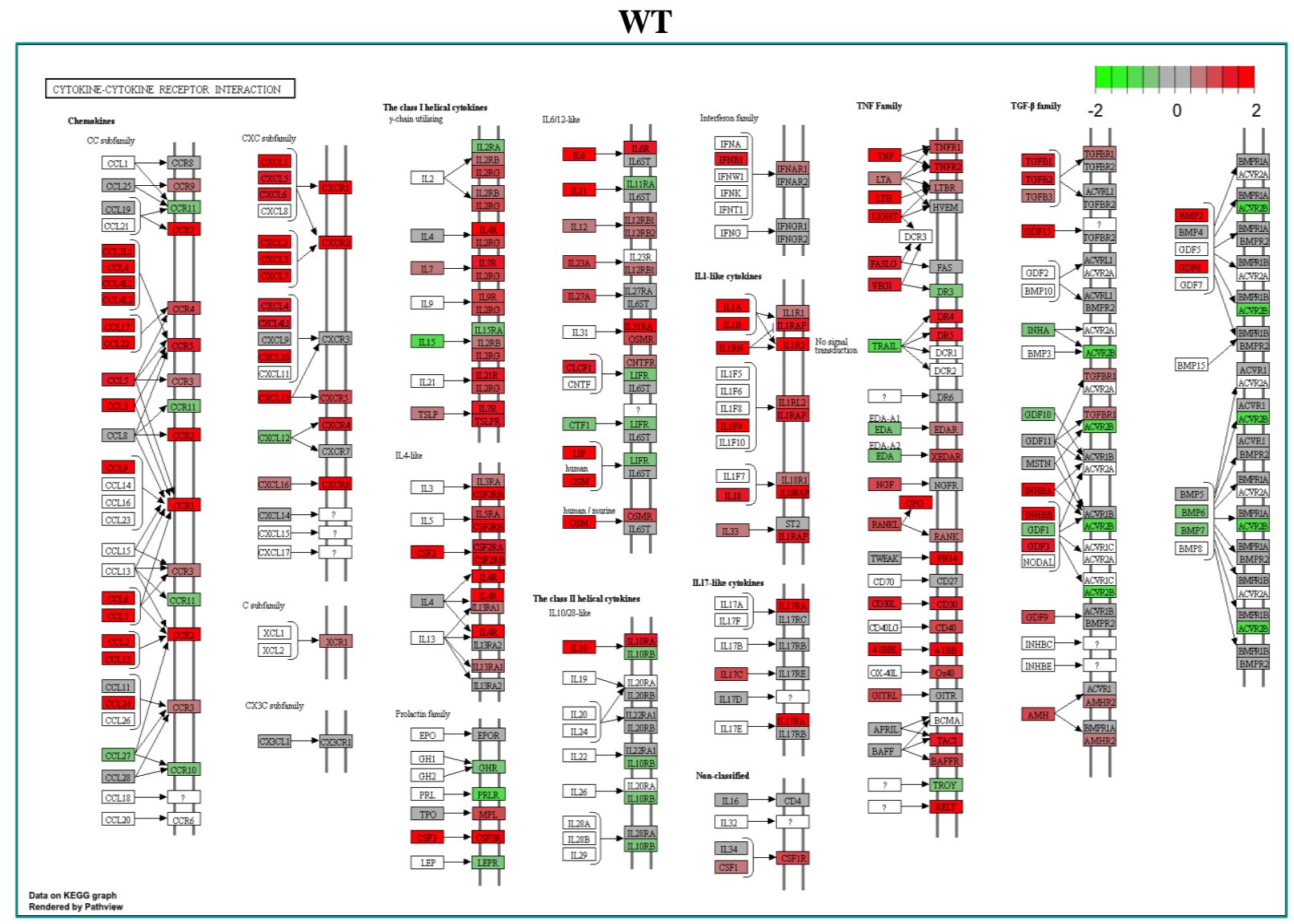

\section{STAT1-F77A}

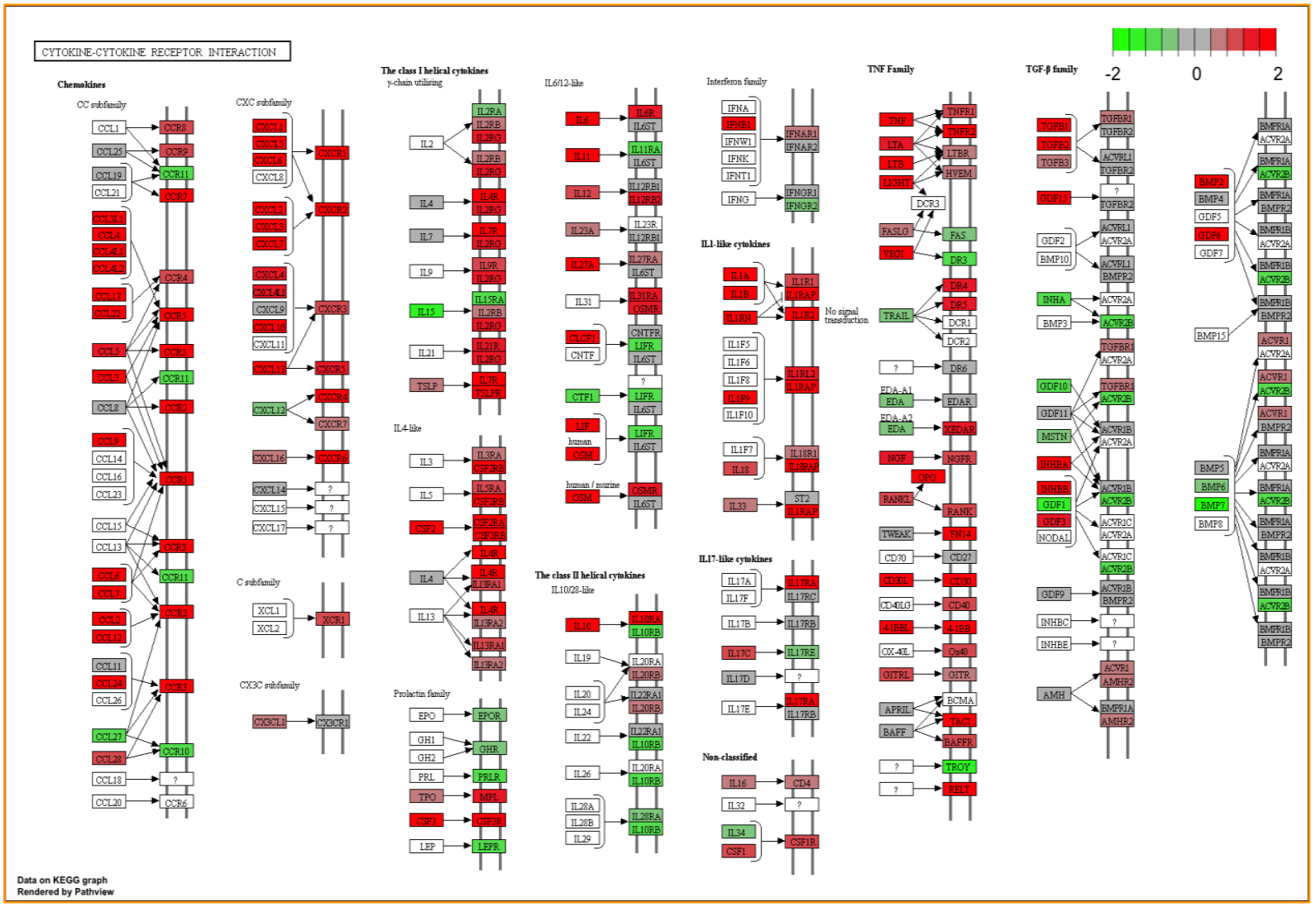

Figure 17: Pathview of the "cytokine-cytokine receptor interaction" KEGG pathway in wildtype and STAT1-F77A mice following LAD ligation. 


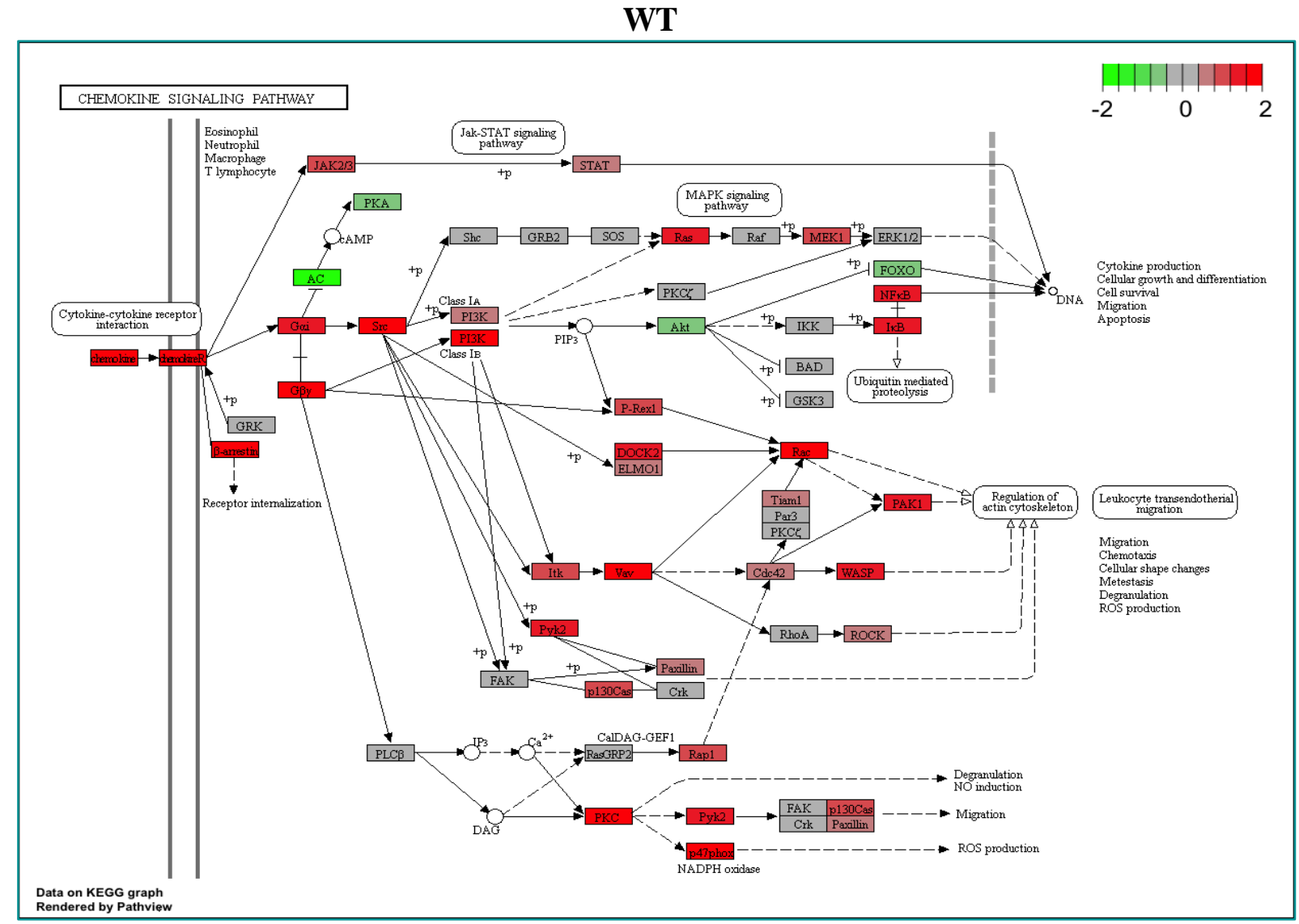

STAT1-F77A

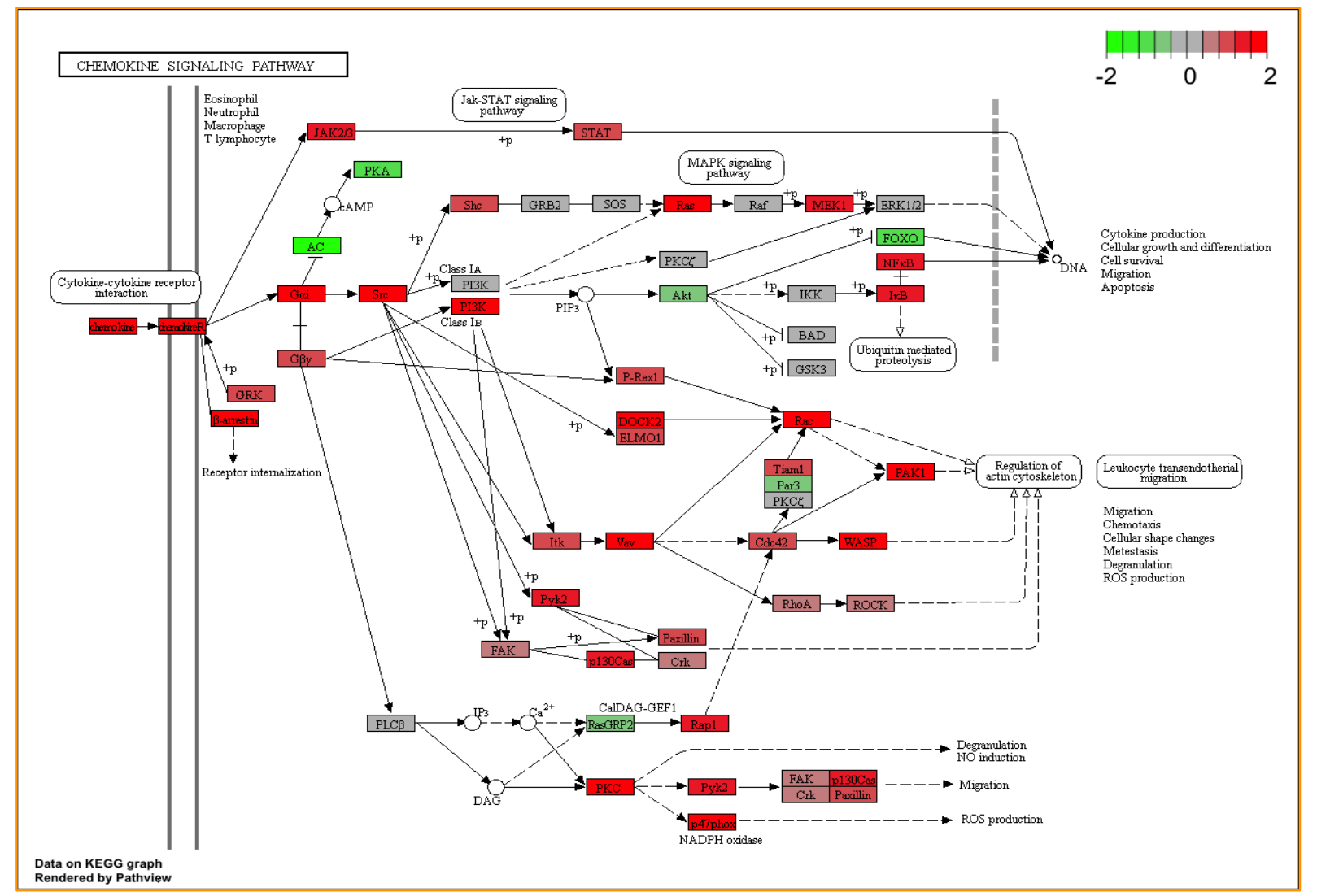

Figure 18: Pathview of the "chemokine signalling" KEGG pathway in wild-type and STAT1F77A mice following LAD ligation. 


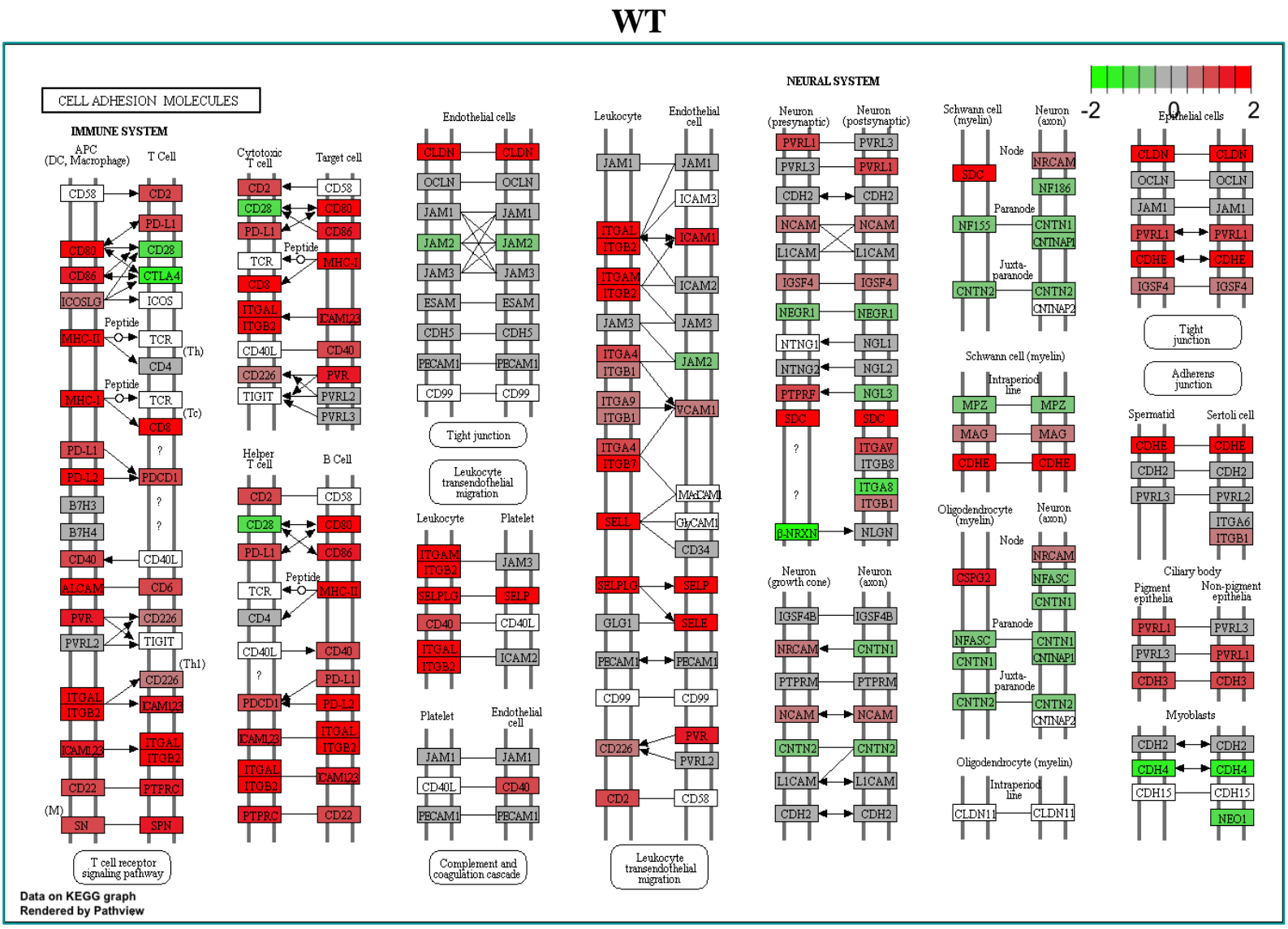

STAT1-F77A

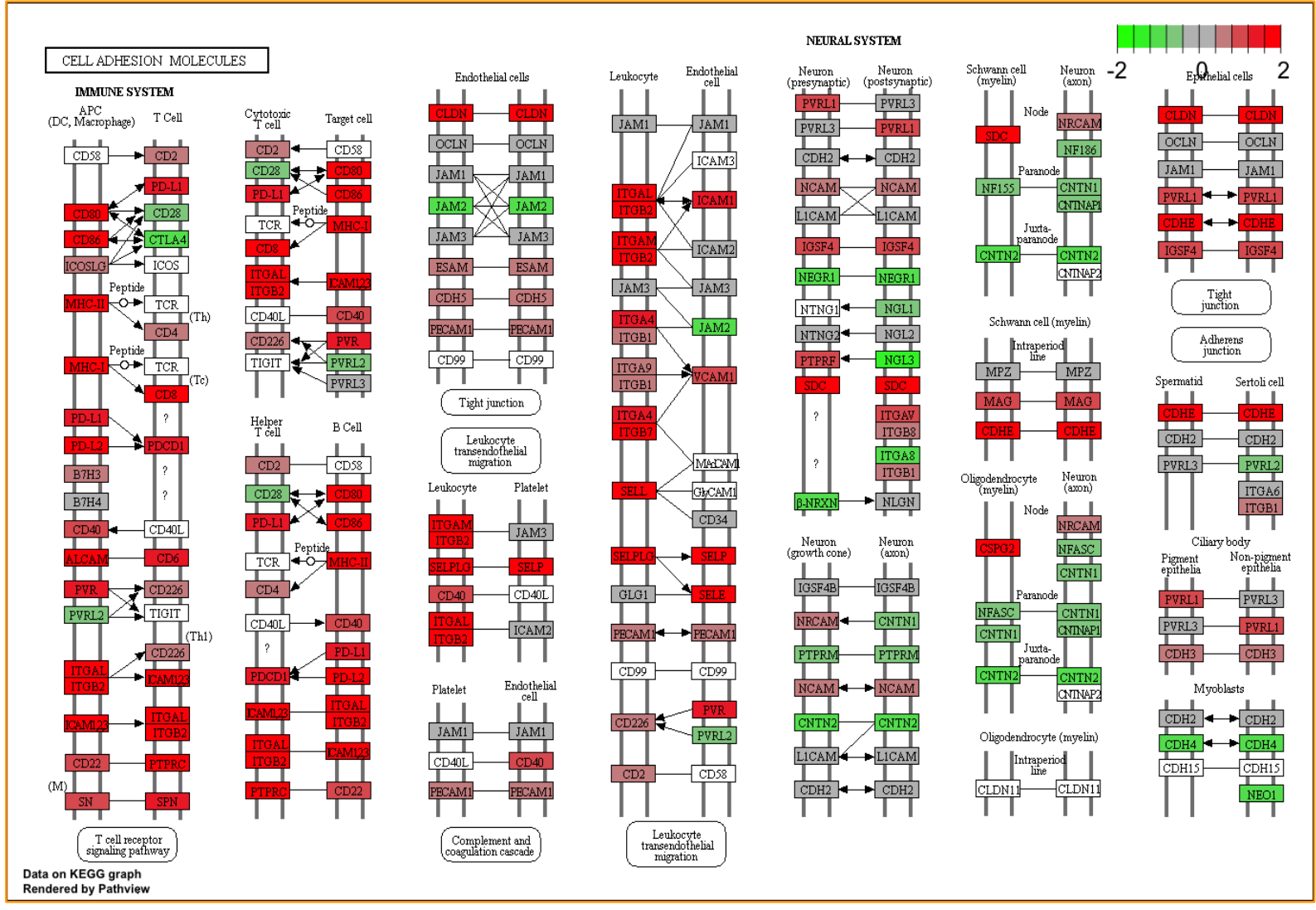

Figure 19: Pathview of the "cell adhesion molecules (CAMs)" KEGG pathway in the two STAT1 genotypes following myocarduial infarction. 

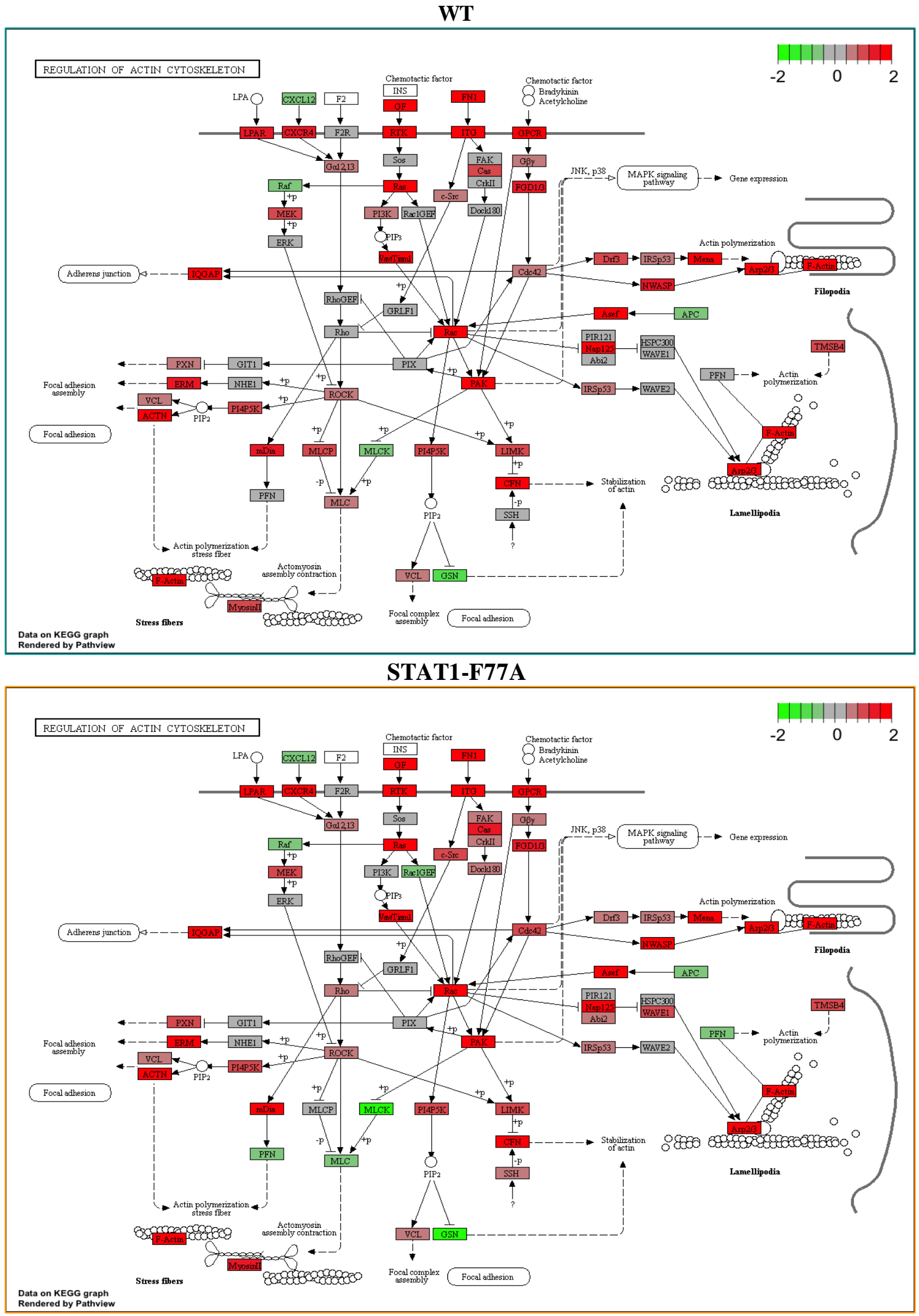

Figure 20: Pathview of the "regulation of actin cytoskeleton" KEGG pathway in mice with with wild-type or mutant Stat allele following LAD ligation. 



Figure 21: Pathview of the "leukocyte transendothelial migration" KEGG pathway in the two Stat1 genotypes following LAD ligation. 


\subsection{Identification of signal pathways and cellular processes differentially regulated after}

\section{LAD ligation}

Since signal transduction pathways transmit extracellular stimuli inside the cell thereby regulating the expression of genes with important physiological functions, we performed differential expression analysis to identify the most significantly enriched signal pathways. Signal transduction pathways that were associated with myocardial infarction and were genotype-independent are the following: PI3K-Akt signalling (Figure 23), JAK-STAT signalling (Figure 24), TNF signalling (Figure 25), NF- $\mathrm{KB}$ signalling (Figure 26), NOD-like receptor signalling (Figure 27), MAPK signalling, osteoclast differentiation, HIF-1 signalling, TGF- $\beta$ signalling, Rap1 signalling, RIG-I-like receptor signalling, Hippo signalling, p53 signalling, and cytosolic DNA-sensing pathway.

Differentially regulated signaling pathways in WT and F77A (MI vs sham)

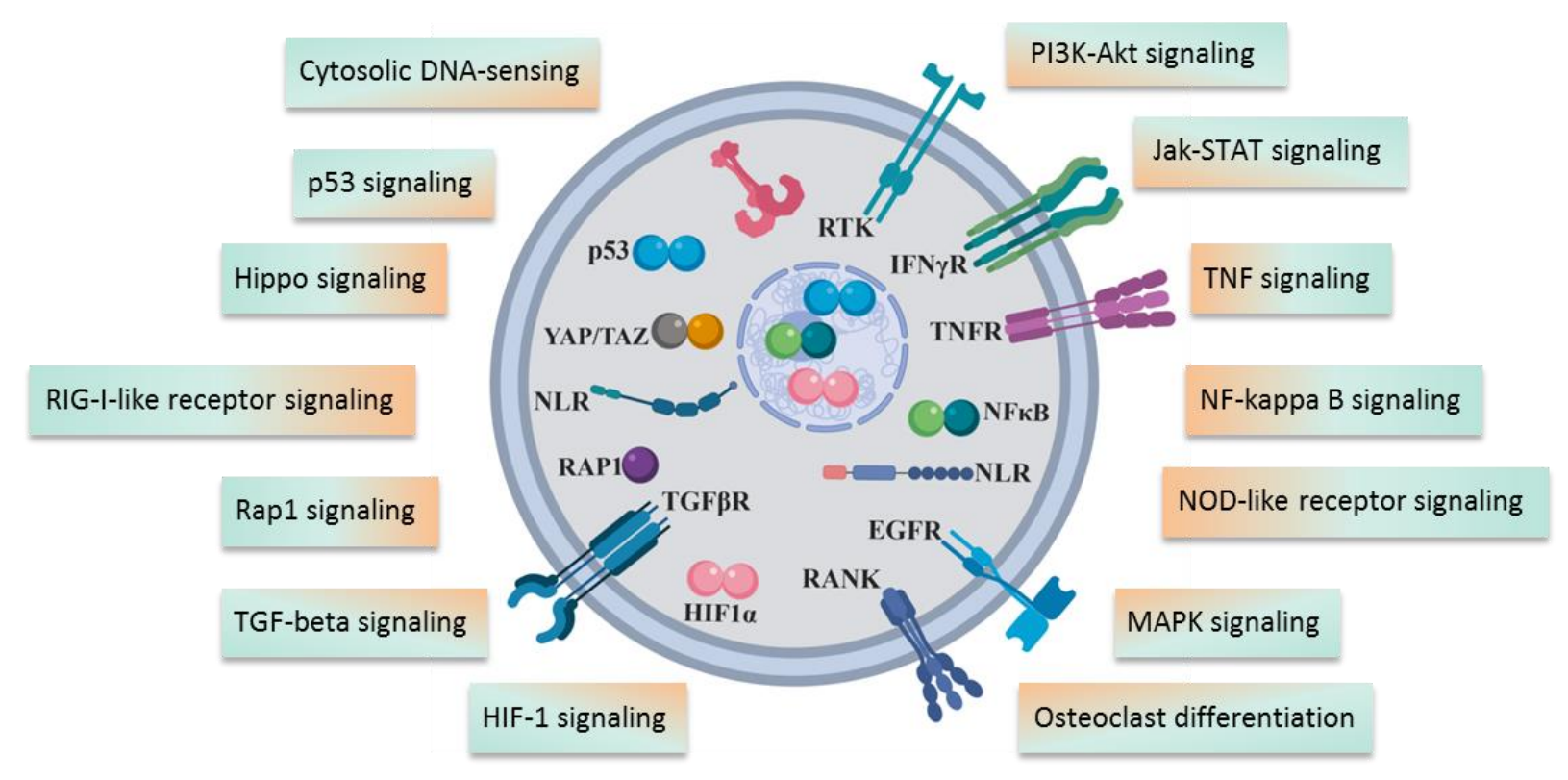

Figure 22: List of KEGG immune responses and signal transduction pathways that are differentially regulated in between STAT1-F77A and wild-type mice. 


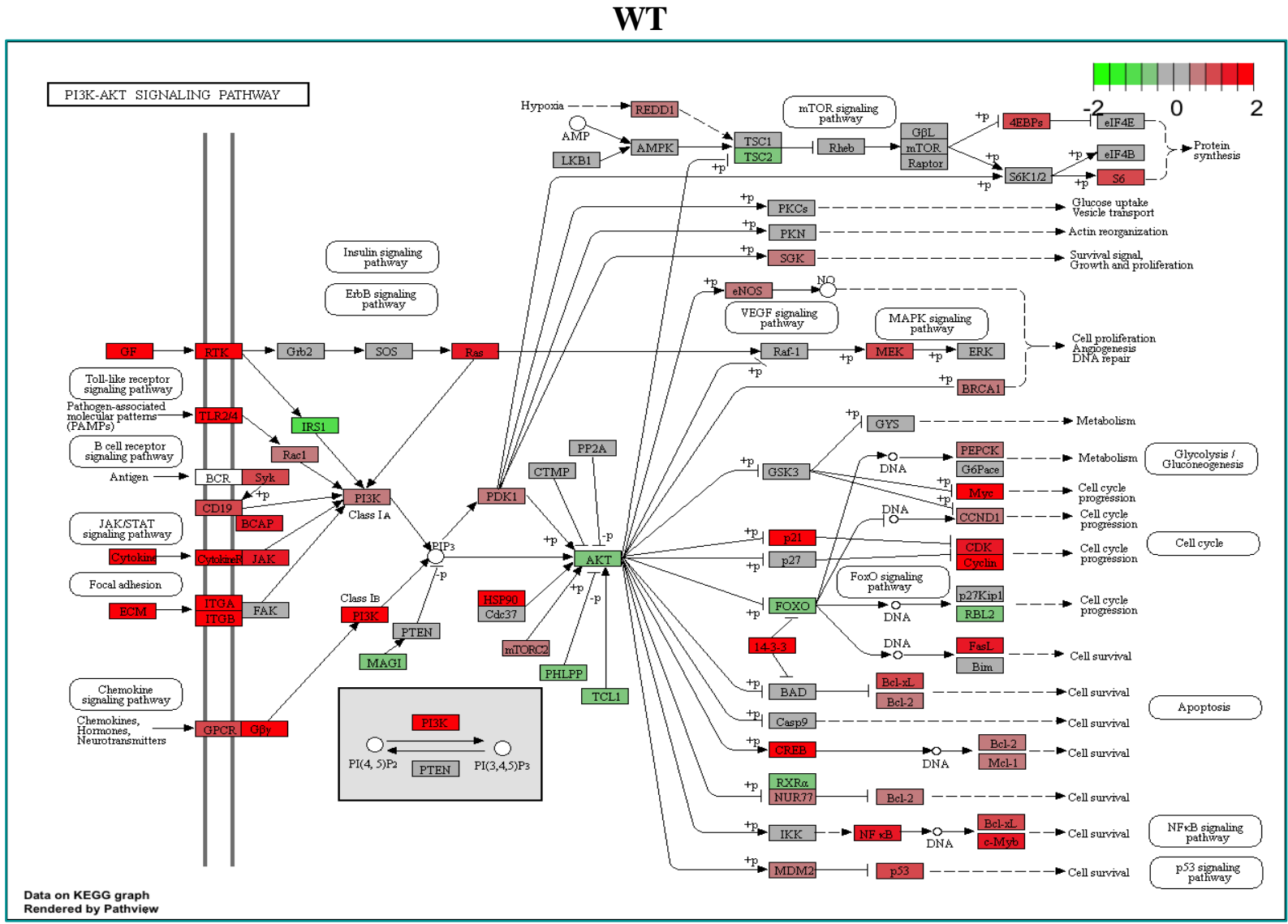

\section{STAT1-F77A}

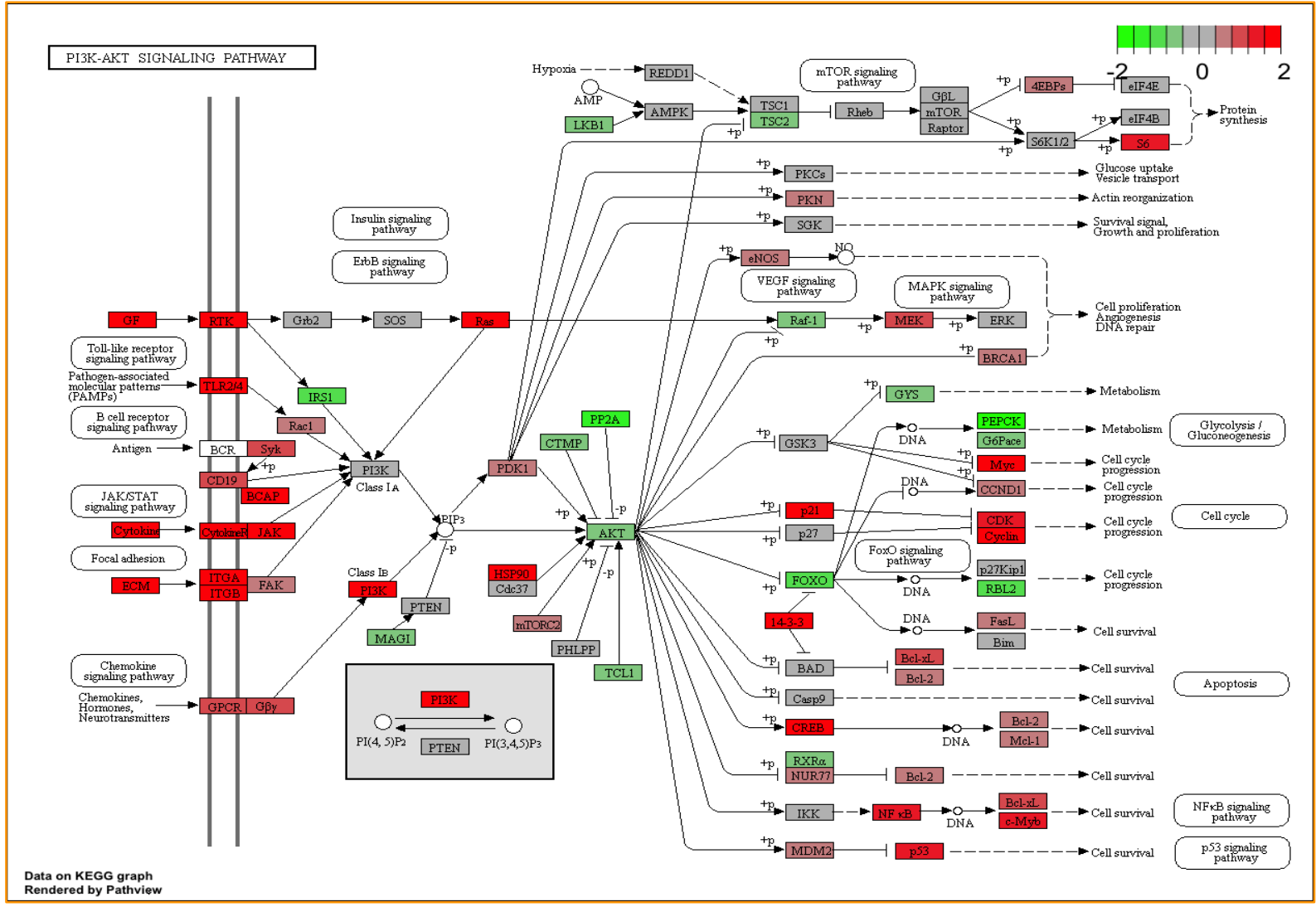

Figure 23: Pathview of the "PI3K-Akt signalling" KEGG pathway in wild-type and STAT1F77A mice following LAD ligation. 


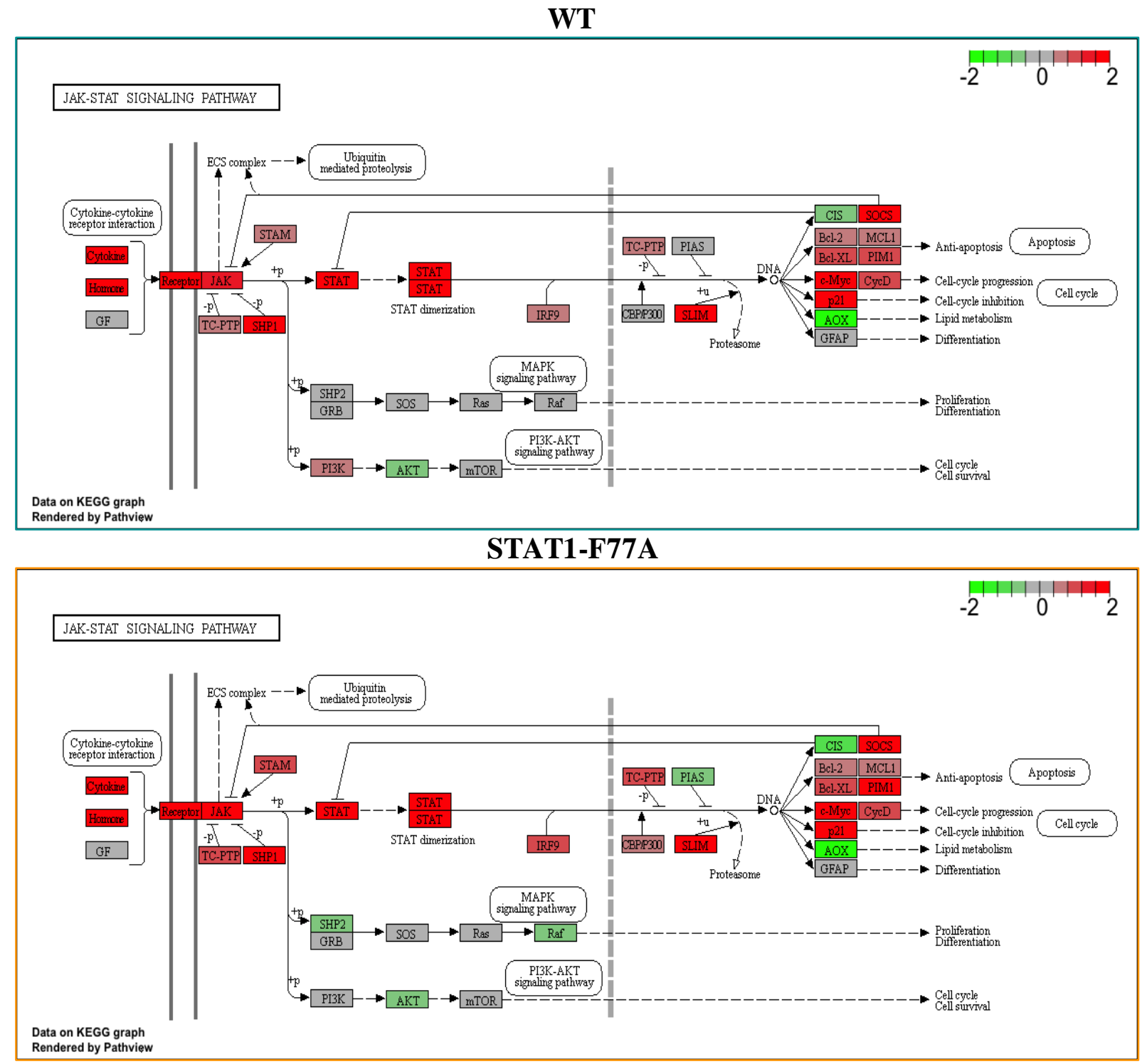

Figure 24: Pathview of the "Jak-STAT signalling" KEGG pathway in wild-type and STAT1F77A mice following LAD ligation. 


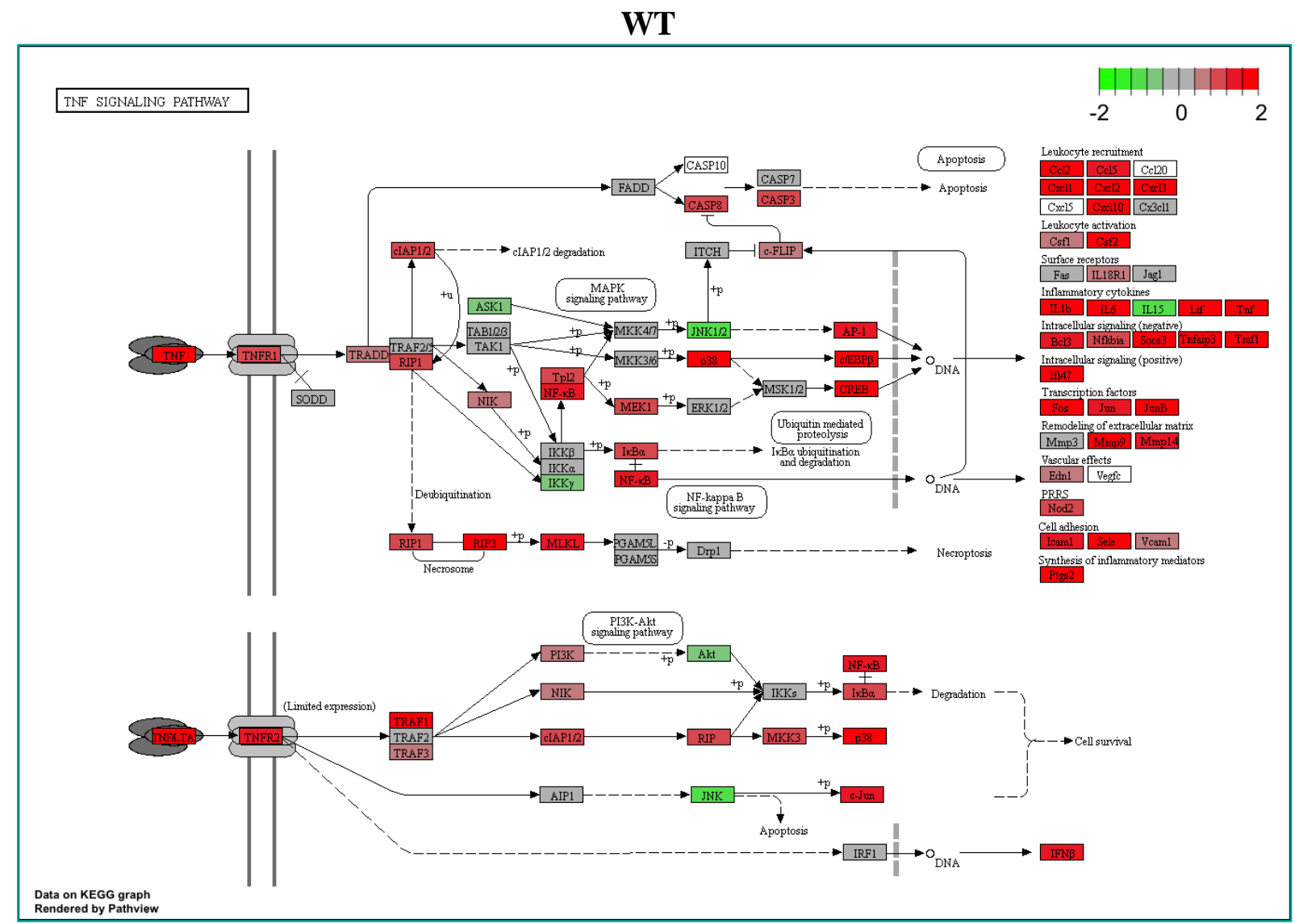

STAT1-F77A

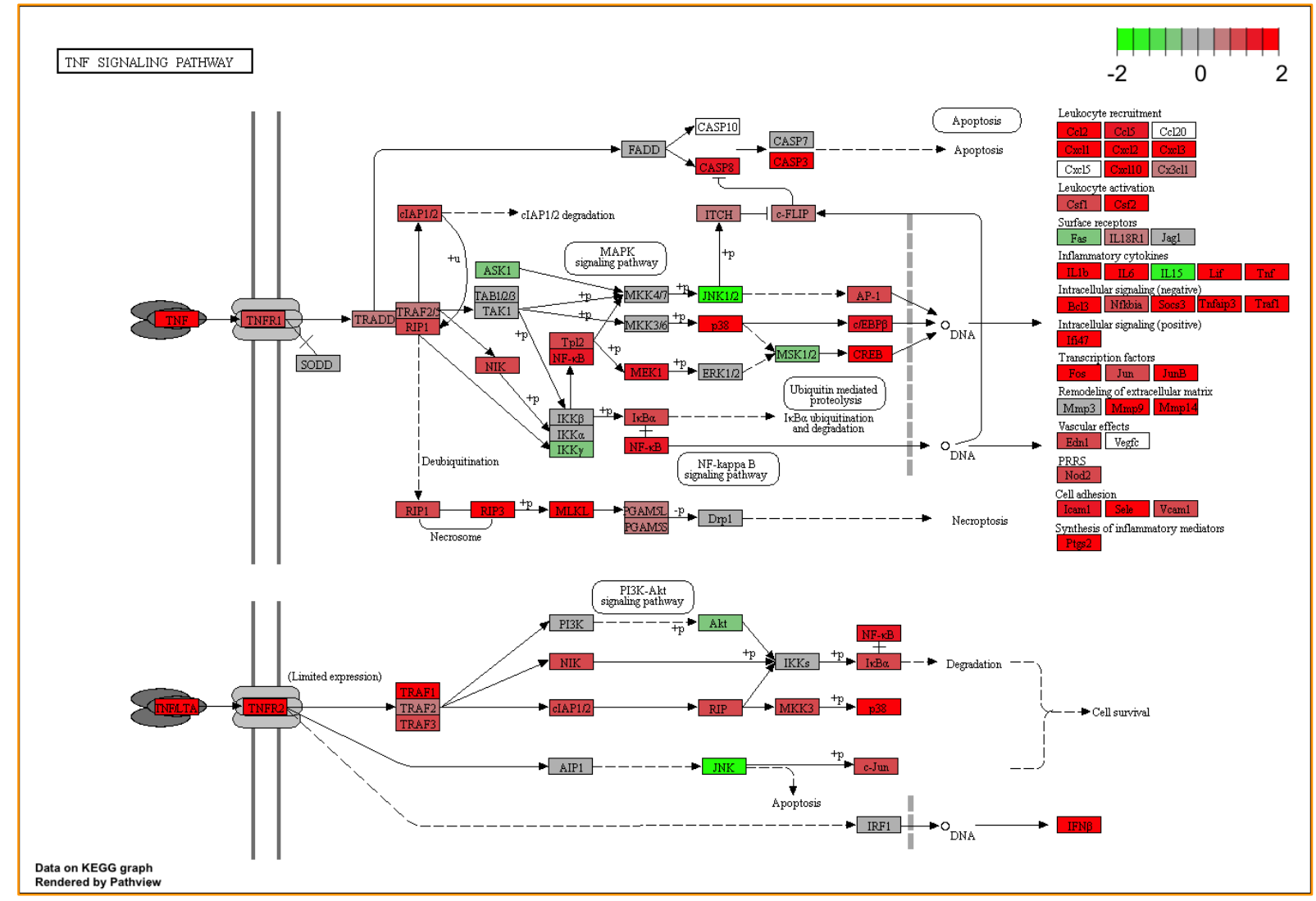

Figure 25: Pathview of the "TNF signalling" KEGG pathway in wild-type and STAT1-F77A mice following LAD ligation. 

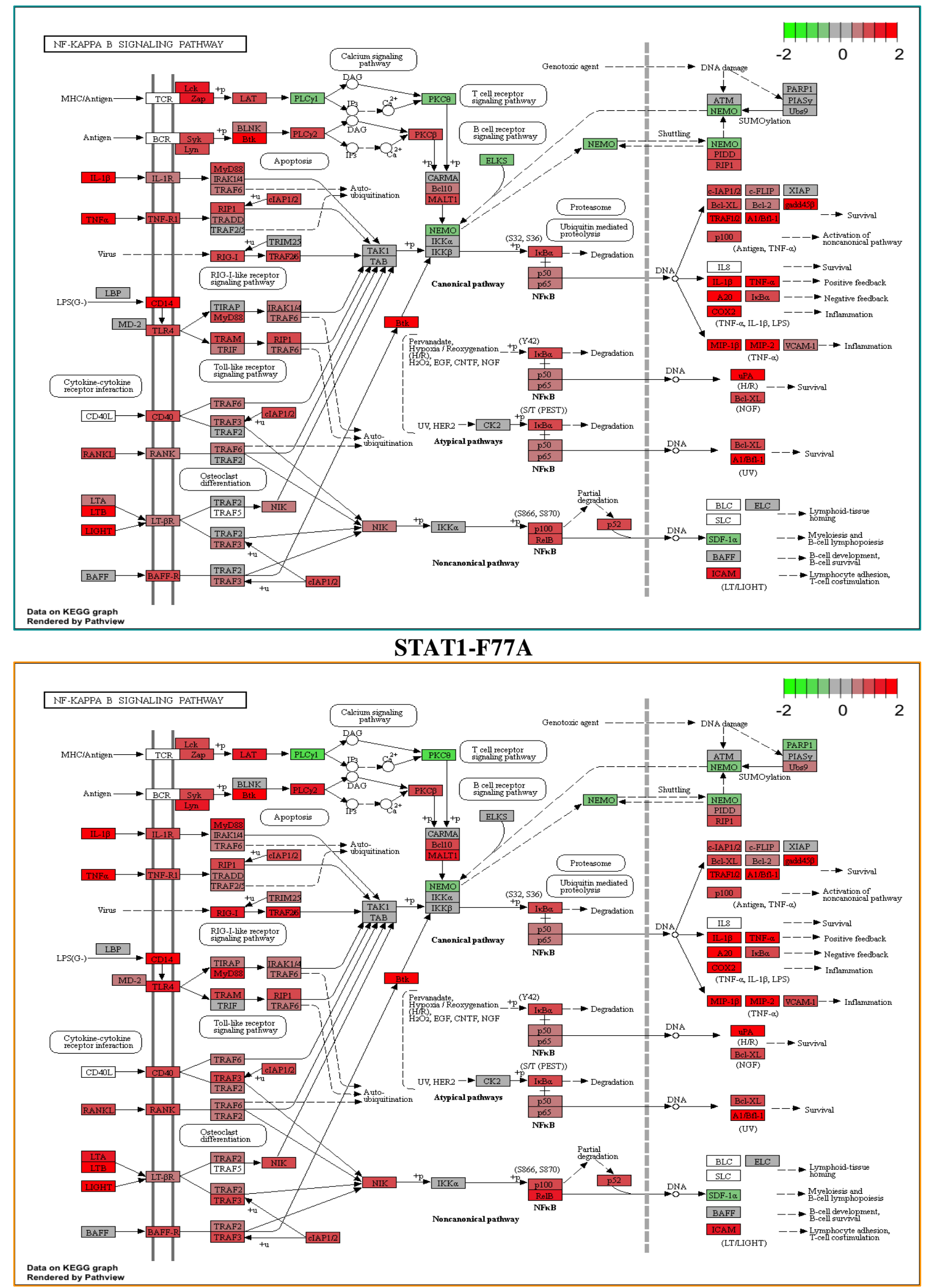

Figure 26: Differential expression of components in the "NF-kB signalling" KEGG pathway in the two Stat1 genotypes following LAD ligation. 
WT

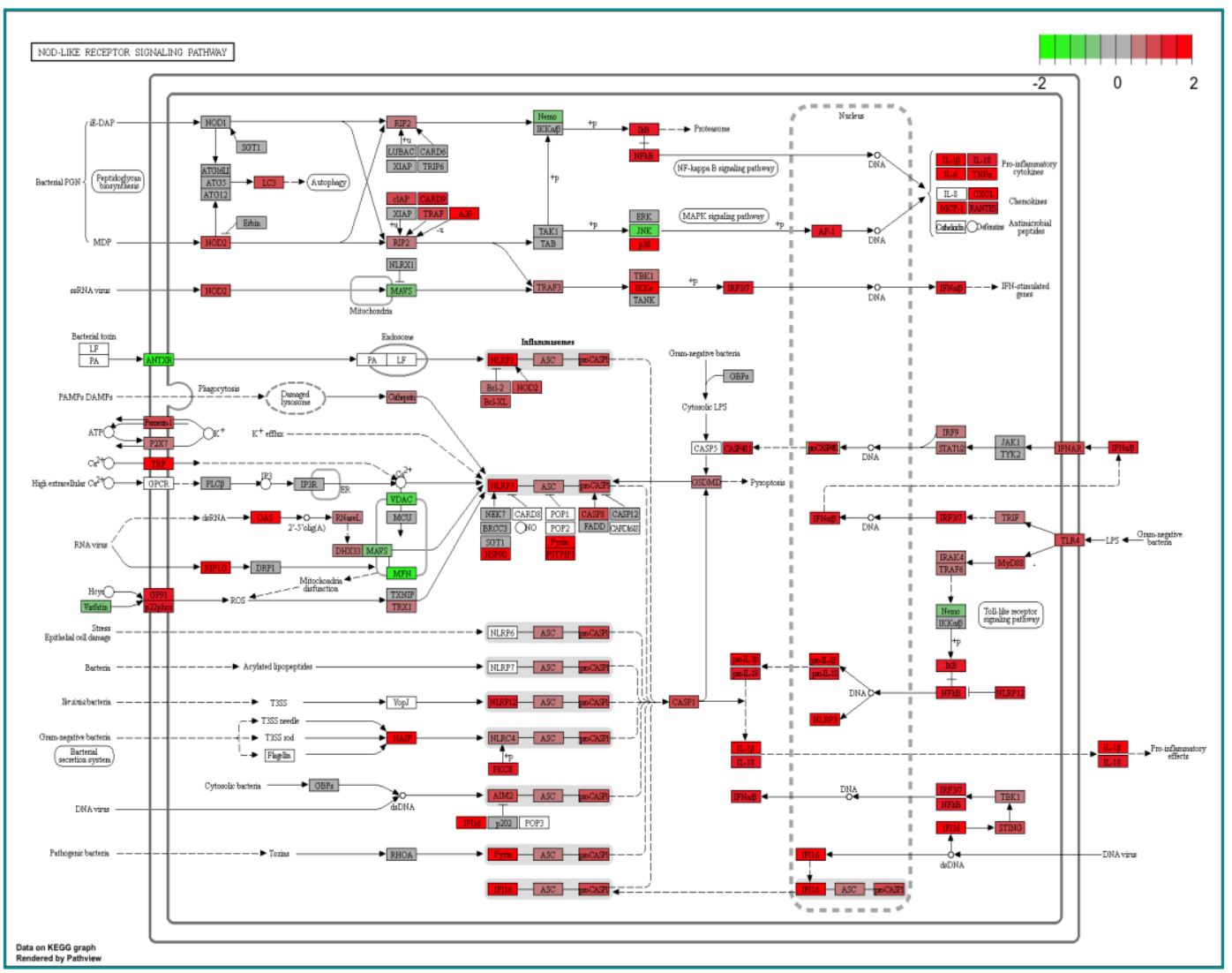

STAT1-F77A

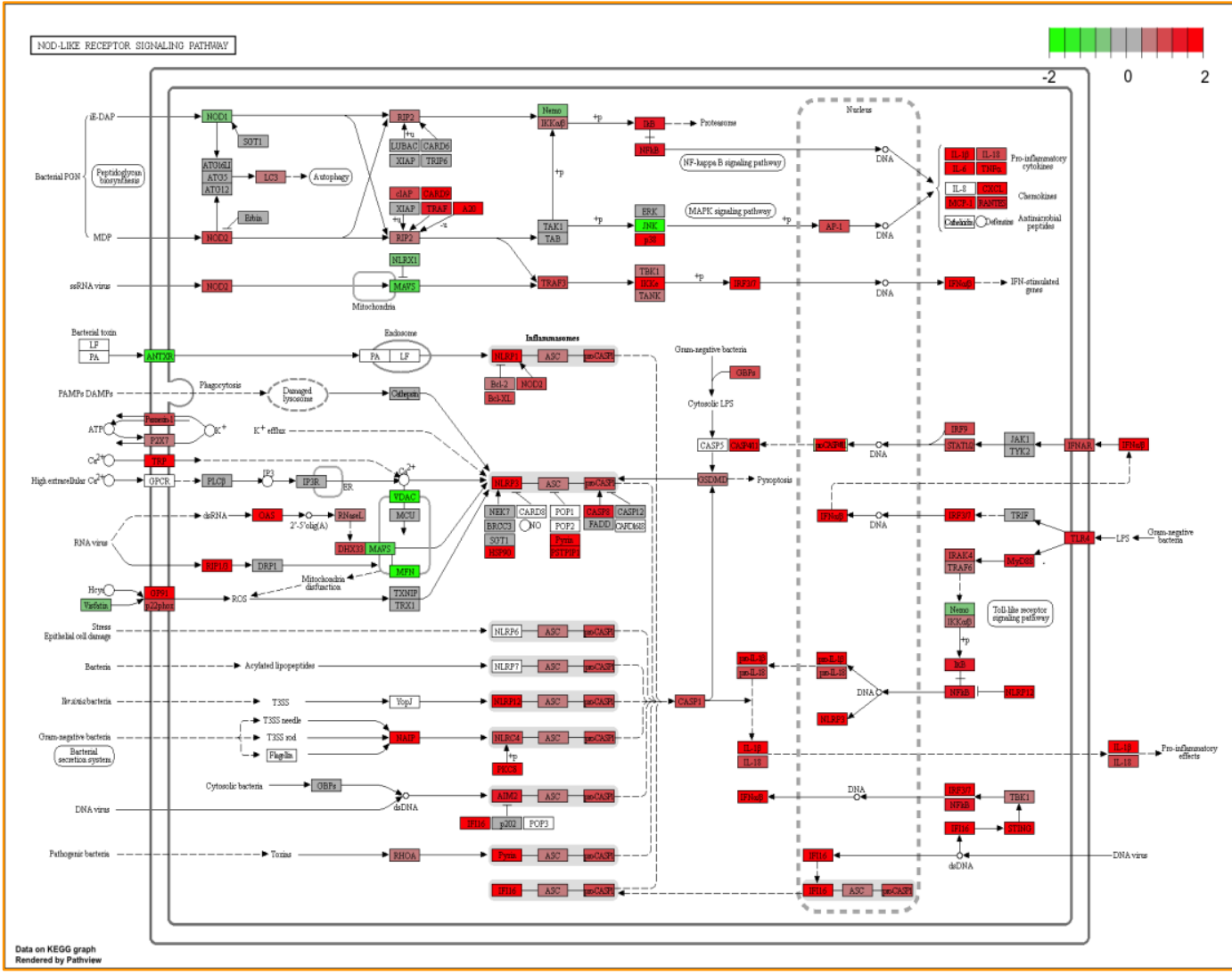

Figure 27: Pathview of the "NOD-like receptor signalling" KEGG pathway in wild-type and STAT1-F77A mice following LAD ligation. 
Additionally, the top significantly perturbed cellular processes associated with MI which are genotype-independent include: endocytosis (Figure 29), cell cycle (Figure 30), cellular senescence (Figure 31), apoptosis (Figure 32), necroptosis (Figure 33), phagosome, peroxisome, spliceosome, protein processing in endoplasmic reticulum, and retrograde endocannabinoid signalling and ribosome biogenesis in eukaryotes. Interestingly, we found cardiac muscle contraction to be uniquely differentially regulated in STAT1-F77A (MI vs sham) comparison only ( $\mathrm{q}$-value $=1.36 \times 10^{-2}$ ), while ribosome pathway was significantly differentially regulated in wild-type (MI vs sham) comparison only (q-value=3.94 x10 ${ }^{-2}$ ).

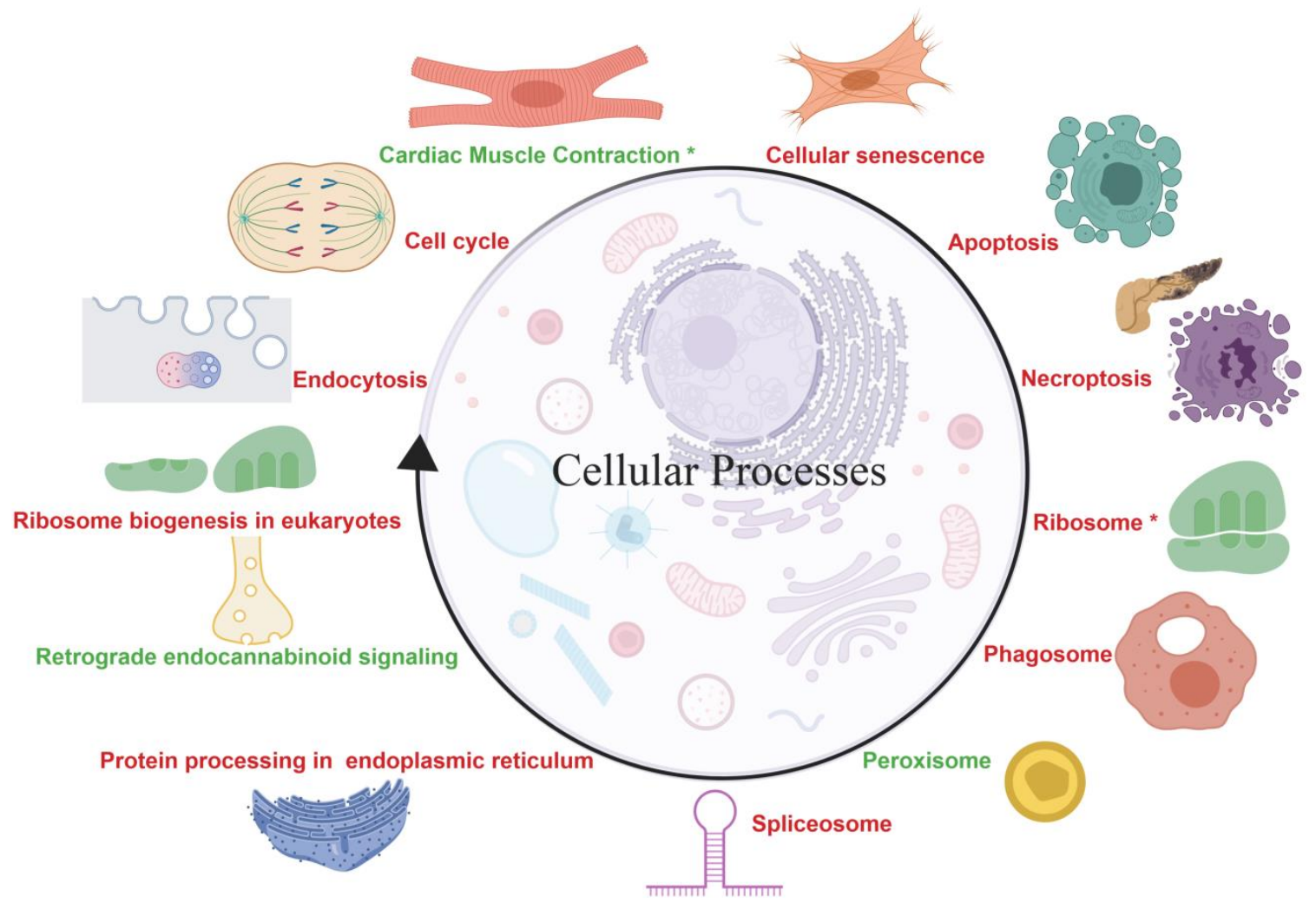

Figure 28: List of KEGG differentially regulated cellular processes following LAD ligation in wild-type and STAT1-F77A mice. Upregulated pathways are displayed in red and downregulated pathways are in green. *Cardiac muscle contraction is uniquely differentially regulated in STAT1-F77A (MI vs sham) comparison only, while ribosome pathway is uniquely differentially regulated in wild-type (MI vs sham) comparison only. 




STAT1-F77A

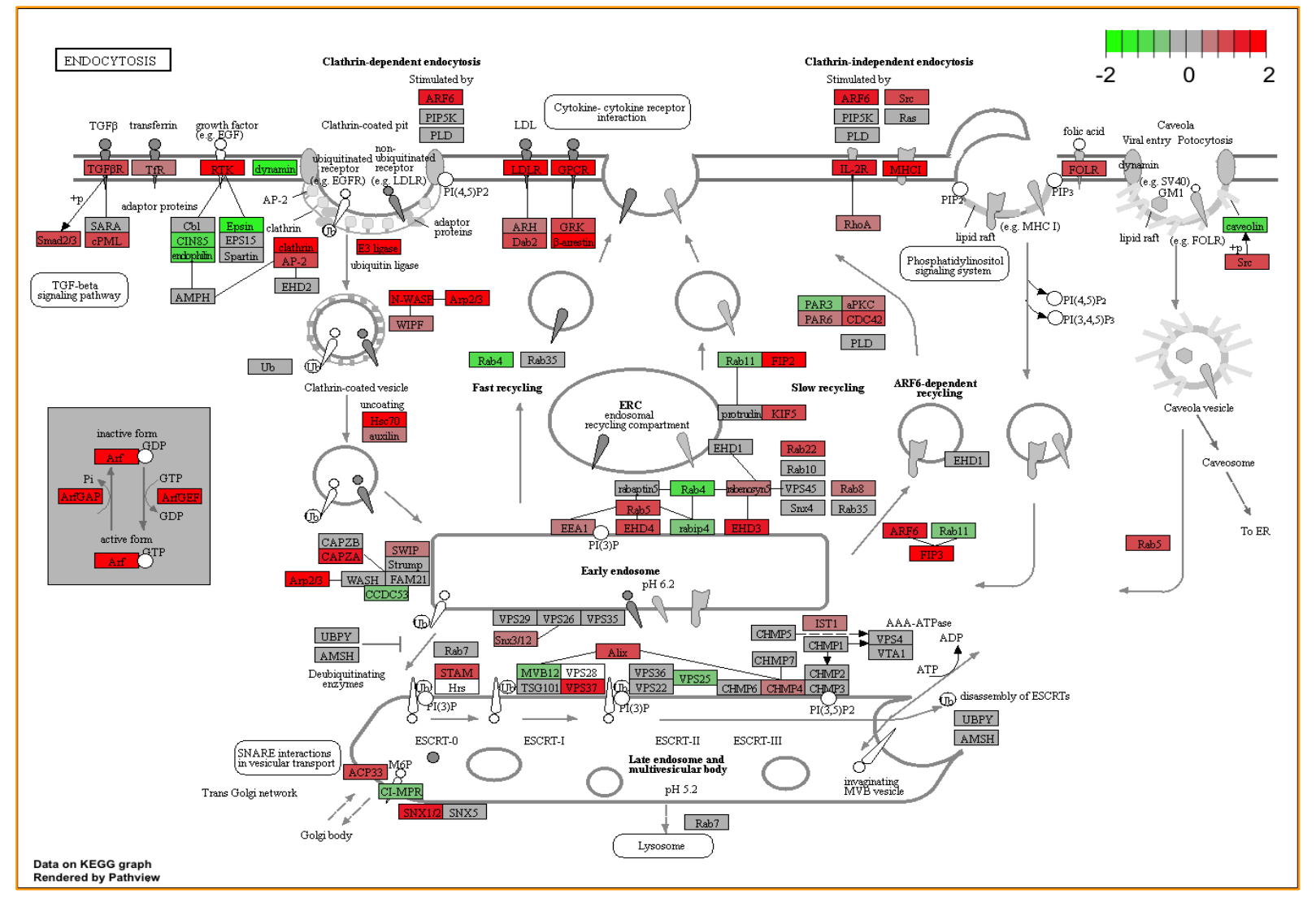

Figure 29: KEGG pathway "endocytosis" in wild-type and STAT1-F77A mice following LAD ligation. 


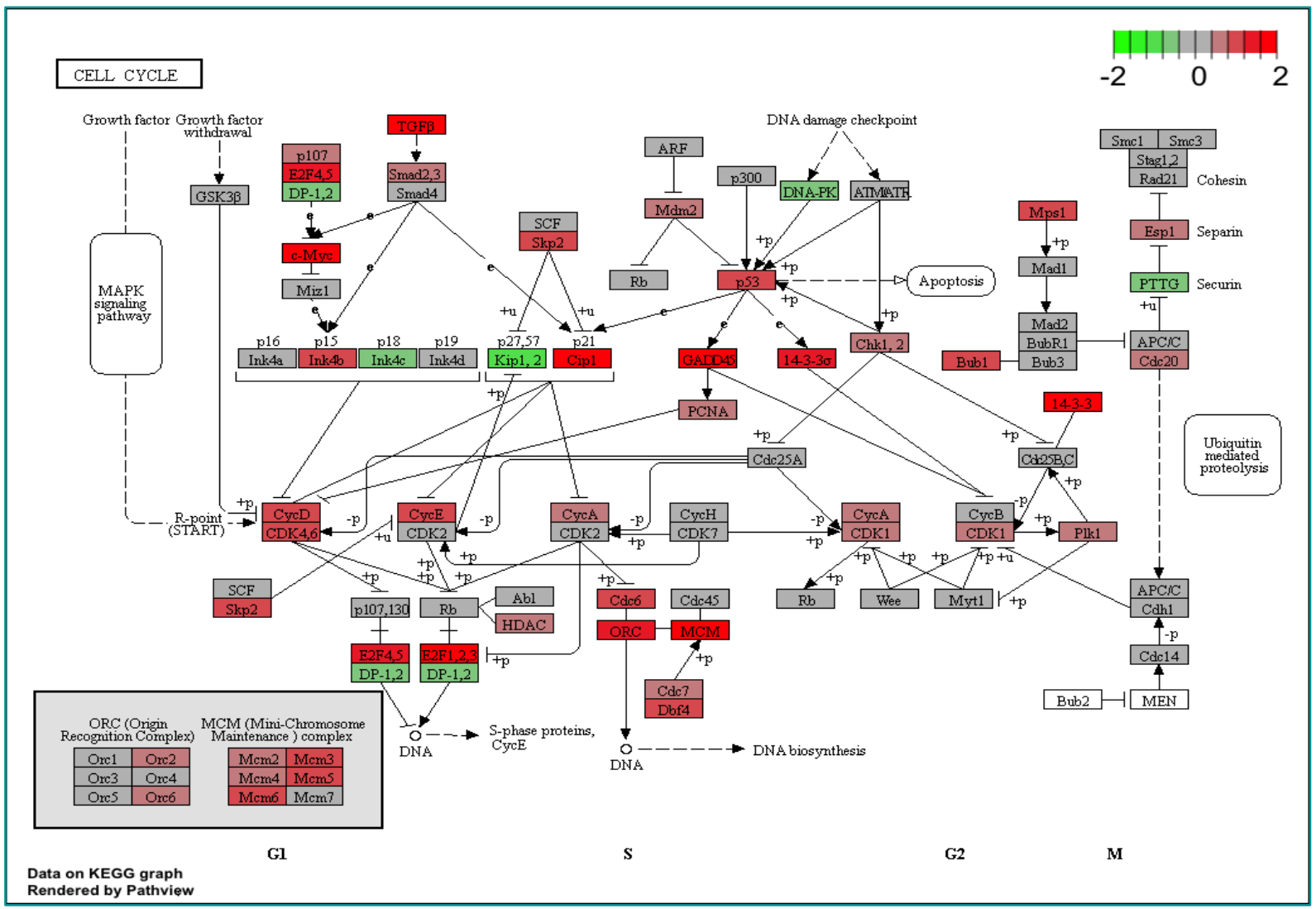

\section{Rendered by Pathview}

\section{STAT1-F77A}

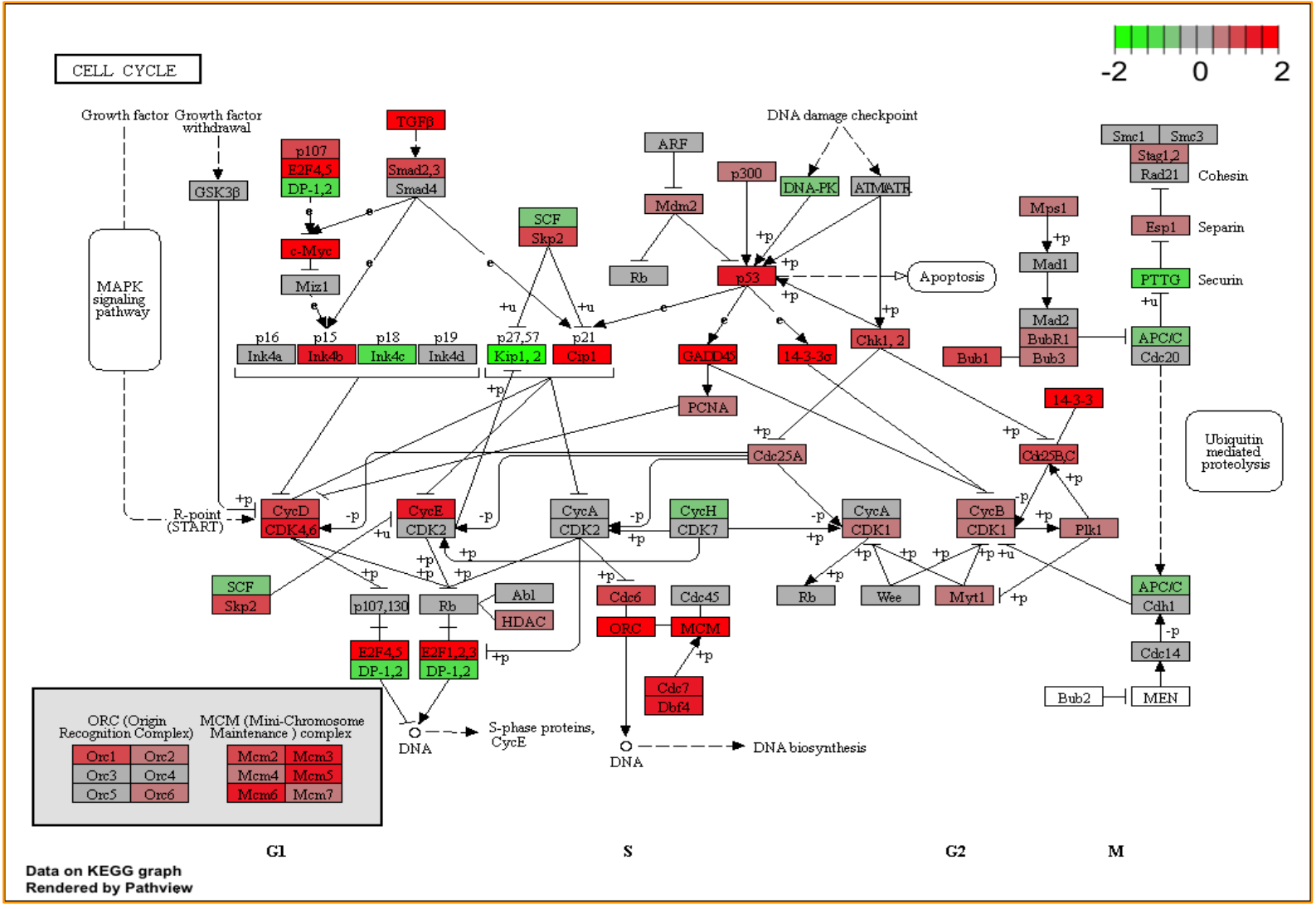

Figure 30: Pathview of the "cell cycle" KEGG pathway in wild-type and STAT1-F77A mice following LAD ligation. 



Figure 31: Pathview of the "cellu lar senescence" KEGG pathway in wild-type and STAT1-F77A mice following LAD ligation. 




Rendered by Pathview

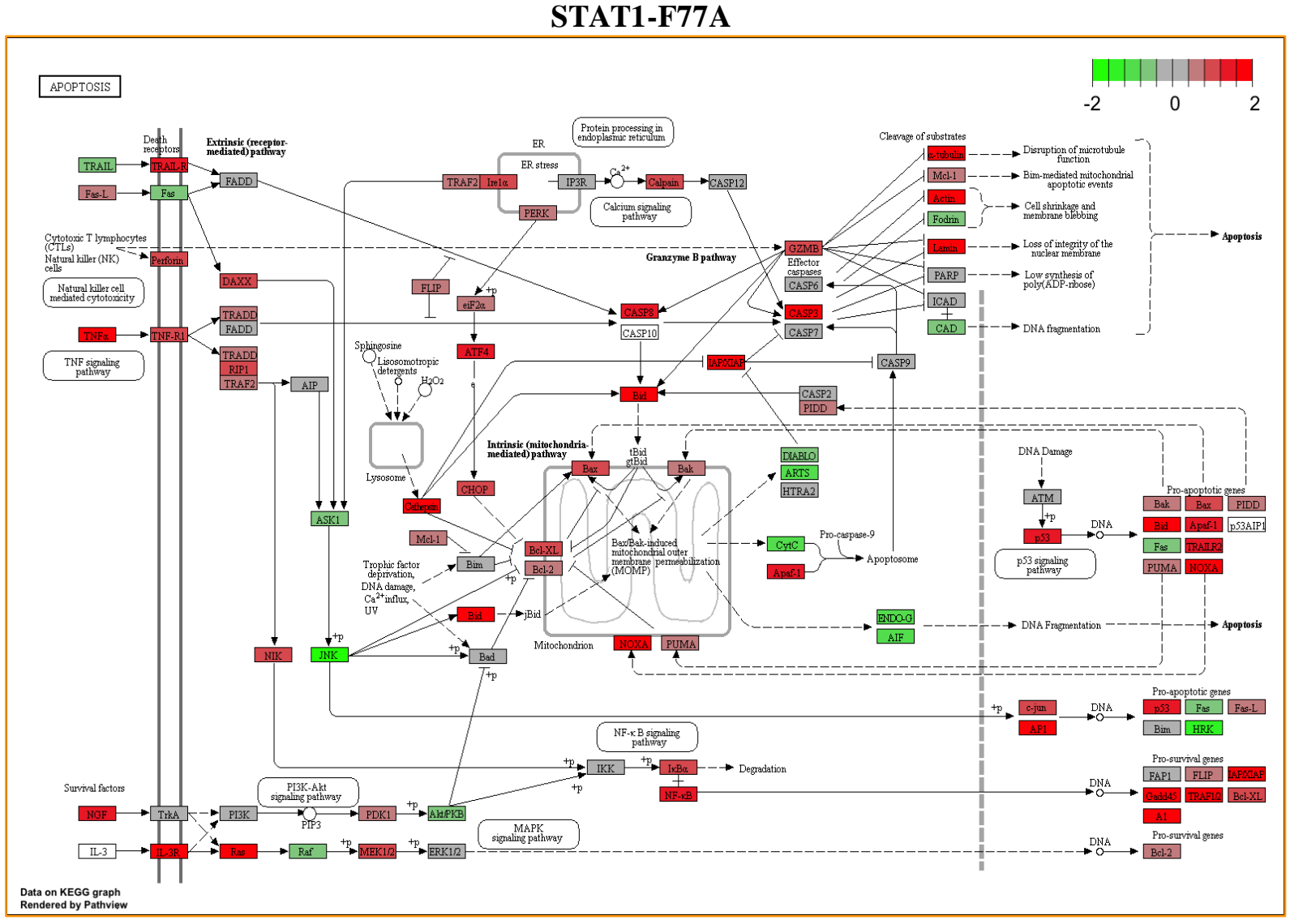

Figure 32: Pathview of the "apoptosis" KEGG pathway in wild-type and STAT1-F77A mice following LAD ligation. 


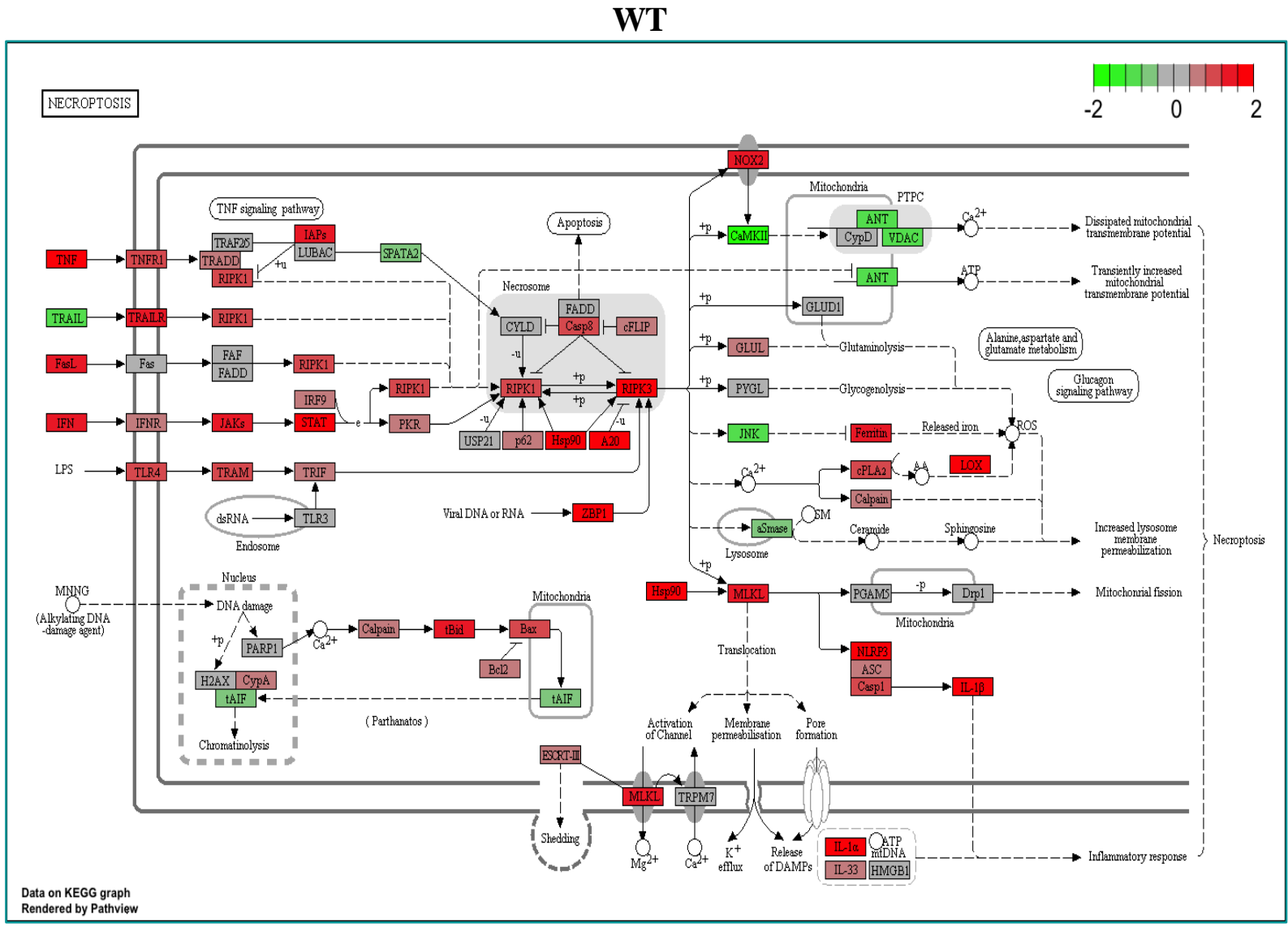

STAT1-F77A

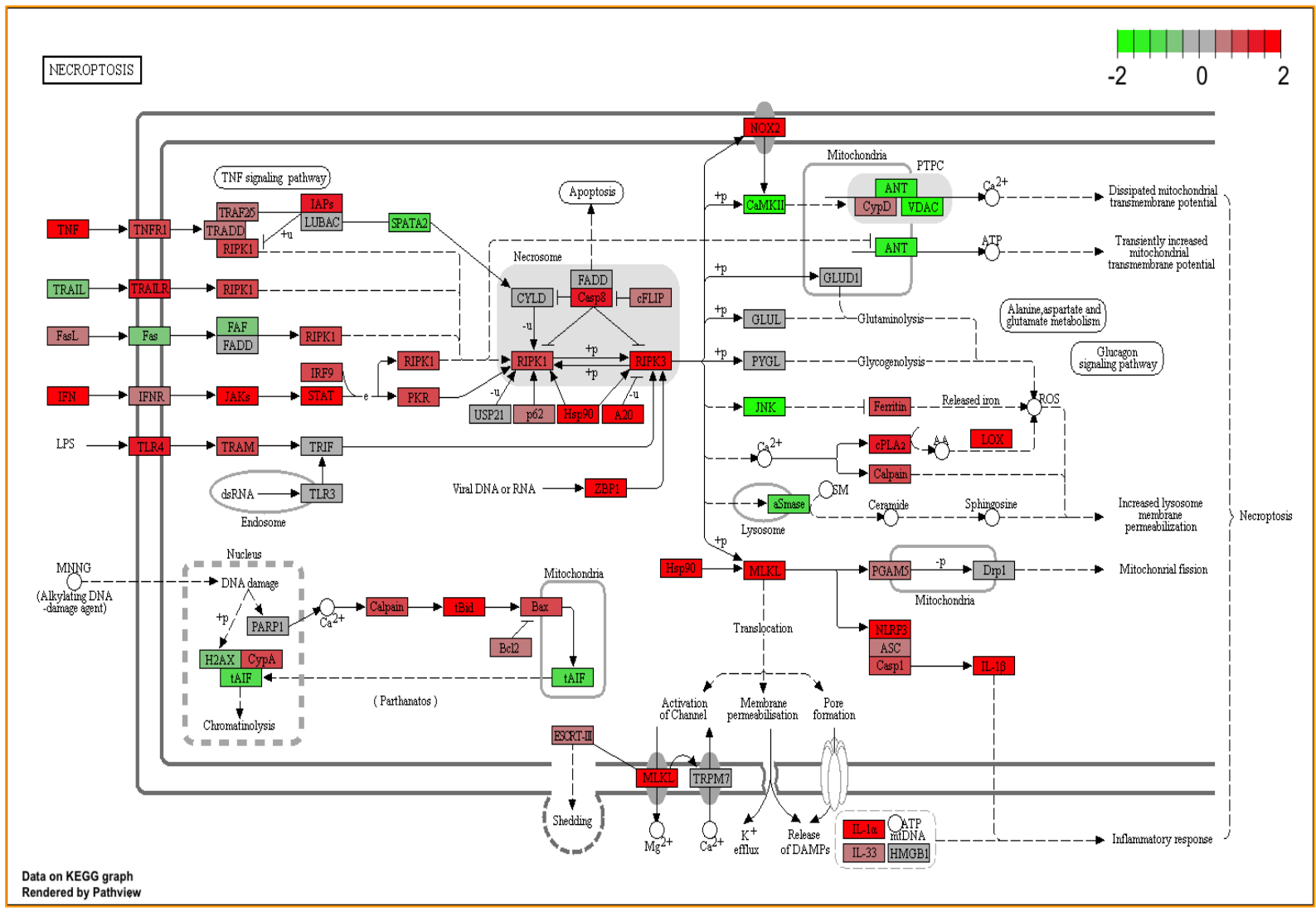

Figure 33: Pathview of the "necroptosis" KEGG pathway in mice expreesing wild-type STAT1 and STAT1-F77A following LAD ligation. 


\subsection{Gene set enrichment analysis revealed changes in metabolic signature following myocardial infarction}

The heart has the capacity to metabolize various types of substrates to synthesize ATP. In this study, we observed that both processes of energy production and energy utilization are dysregulated in the failing heart. Differential analysis revealed downregulation of metabolic pathways, involving oxidative phosphorylation (Figure 34), citrate cycle (TCA cycle) (Figure 35), fatty acid metabolism (Figure 36), fatty acid degradation (Figure 37), propanoate metabolism (Figure 38), tryptophan metabolism, pyruvate metabolism, valine, leucine and isoleucine degradation and glycosaminoglycan biosynthesis in both wild-type and STAT1F77A following myocardial infarction. Whereas lysine degradation was only significantly differentially regulated in wild-type (MI vs sham) comparison, other pathways significantly differentially regulated in STAT1-F77A (MI vs sham) were butanoate metabolism, glyoxylate and dicarboxylate metabolism, carbon metabolism, drug metabolism-cytochrome P450, PPAR signalling pathway, and metabolism of xenobiotics by cytochrome P450. Similarly, performing hierarchical clustering analysis showed that the 1428 DEGs which are downregulated in STAT1-F77A have similar functions, which are involved in the regulation of metabolic pathways related to oxidative phosphorylation, citrate cycle (TCA cycle), fatty acid metabolism, fatty acid degradation, propanoate and tryptophan metabolism, pyruvate metabolism, and valine, leucine and isoleucine degradation. In addition, those genes were associated with glycosaminoglycan biosynthesis, butanoate metabolism, glyoxylate and dicarboxylate metabolism, carbon metabolism, drug metabolism and cytochrome P450, and PPAR signalling pathway (Figure 39). From these data, we hypothesize that the differential gene expression pattern between animals from the two genotypes resulted from a higher immune cell infiltration in STAT1-F77A mice, demonstrating a metabolic shift from oxidative phosphorylation towards glycolysis, which is more prominent in the knock-in mice. 


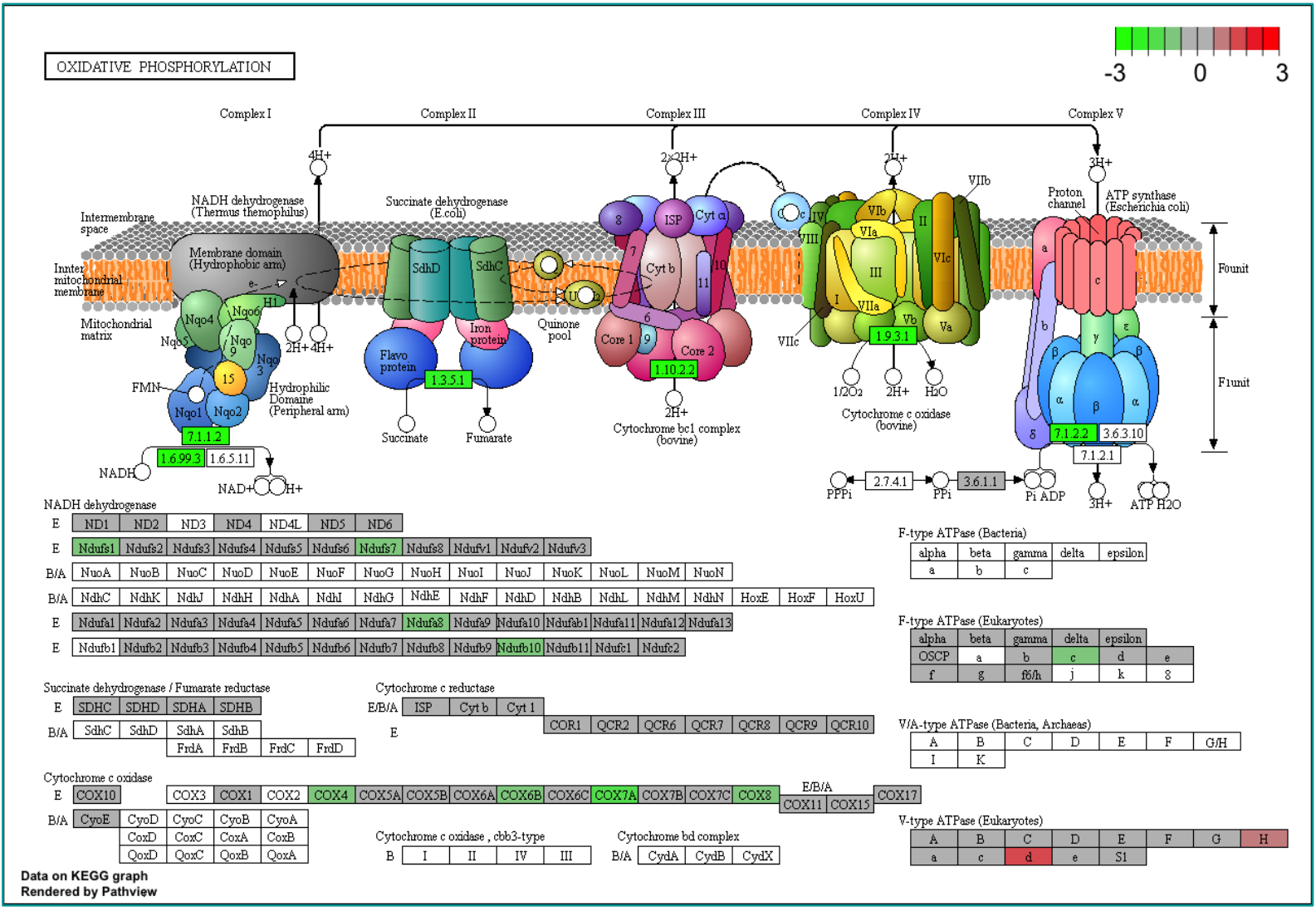

\section{STAT1-F77A}



Figure 34: Pathview of the "oxidative phosphorylation" KEGG pathway in wild-type and STAT1-F77A mice following LAD ligation. 


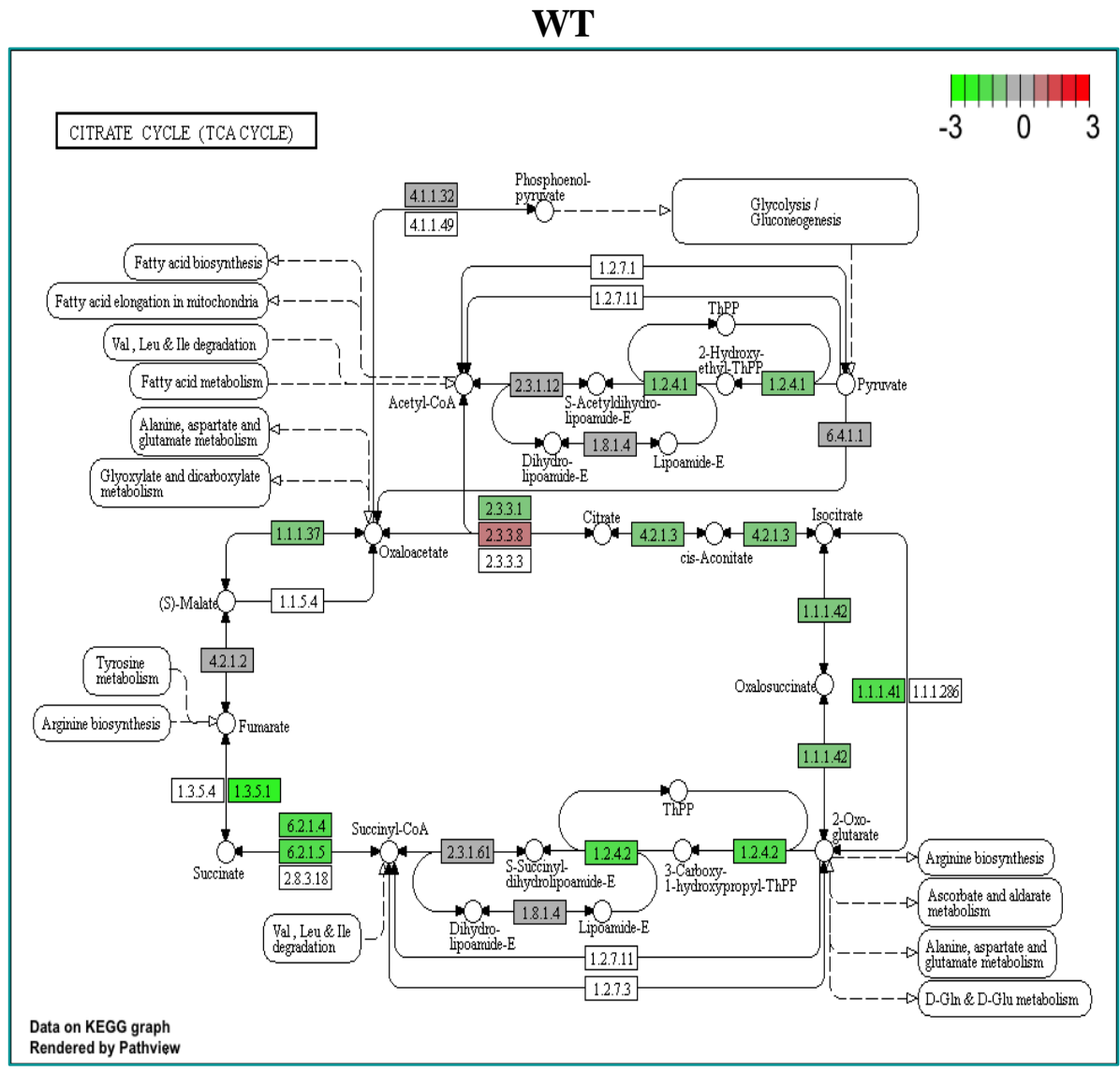

STAT1-F77A

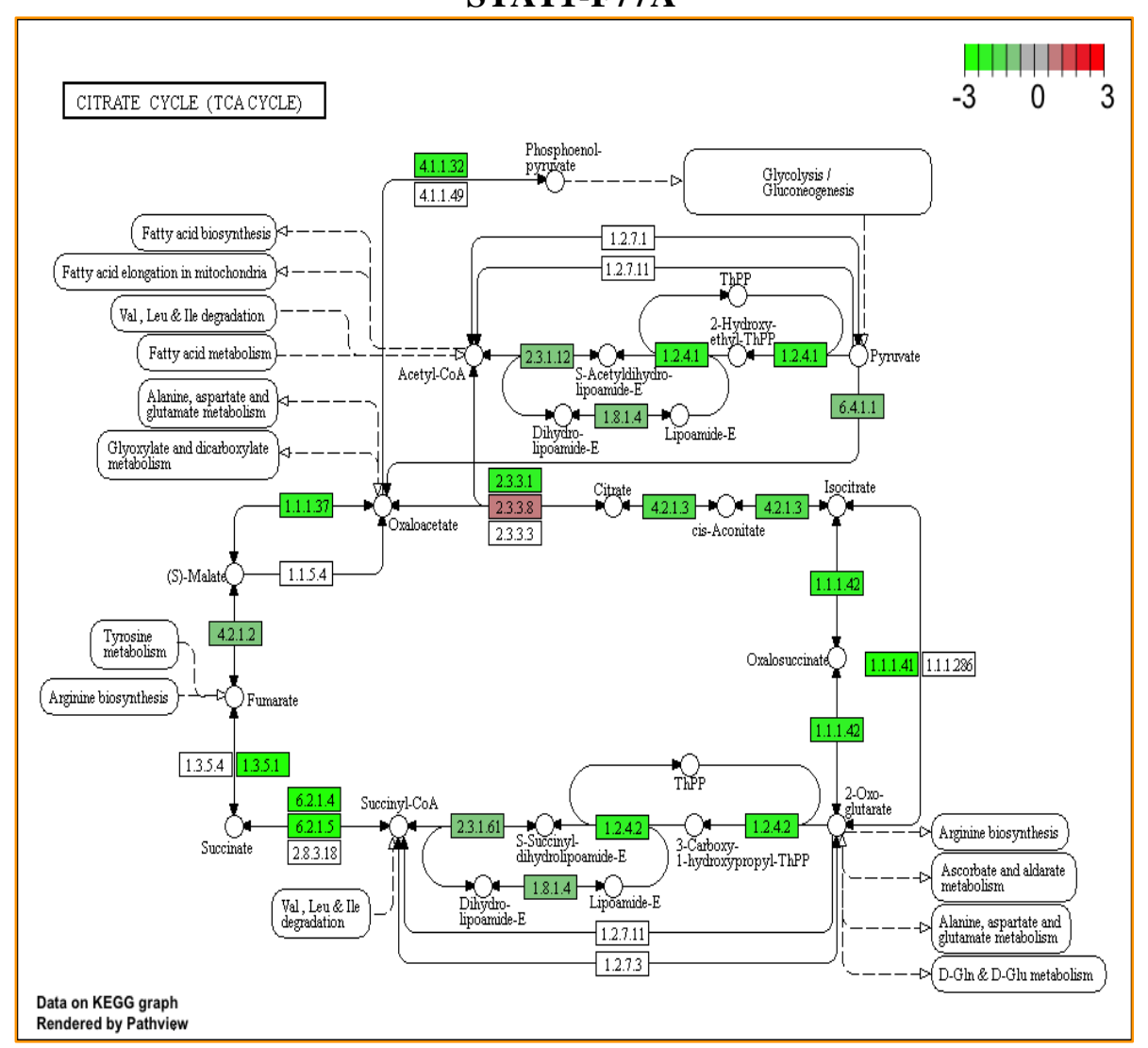

Figure 35: Pathview of the "citrate cycle (TCA cycle)" KEGG pathway in wild-type and STAT1F77A mice following LAD ligation. 
WT

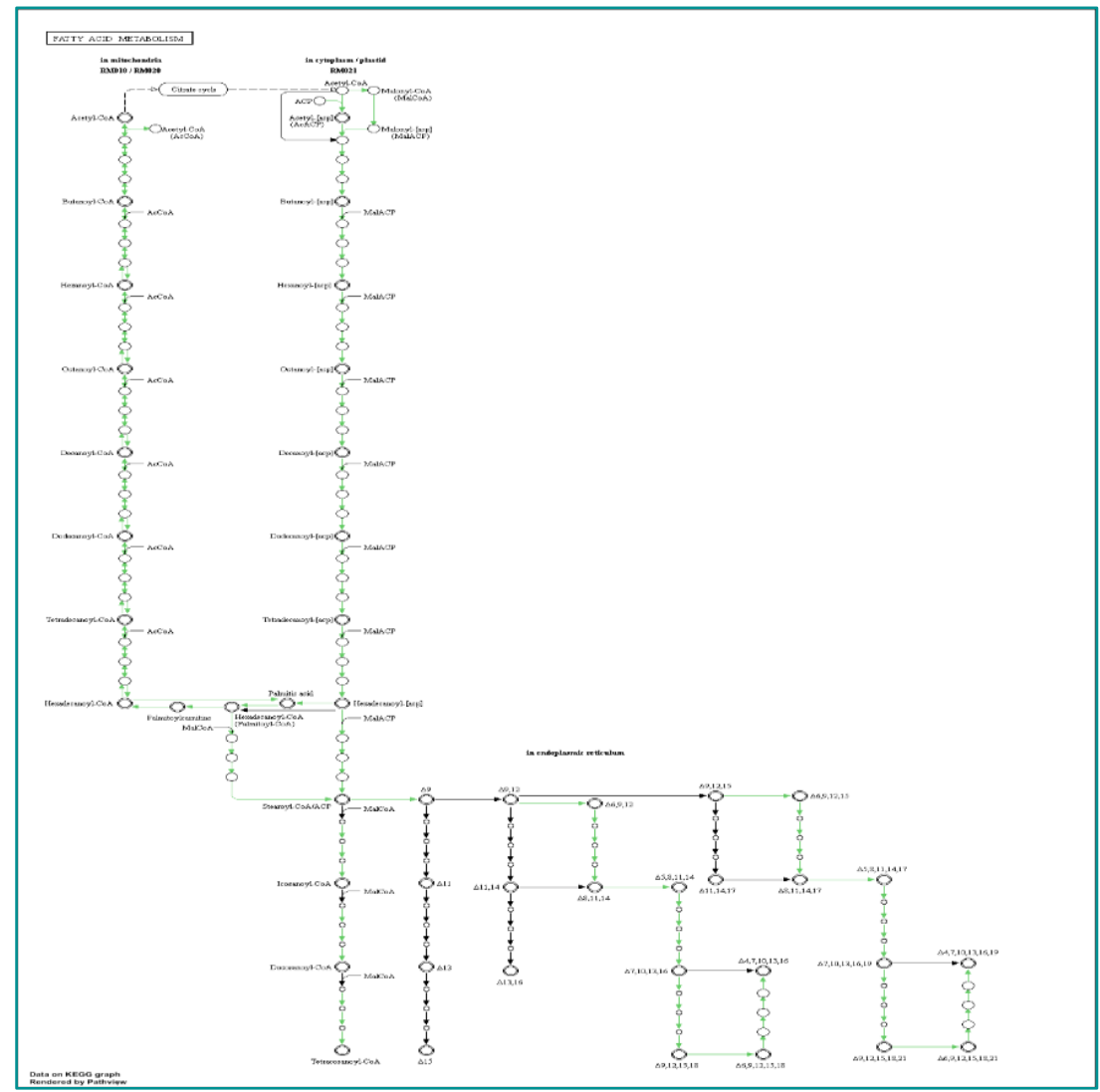

STAT1-F77A

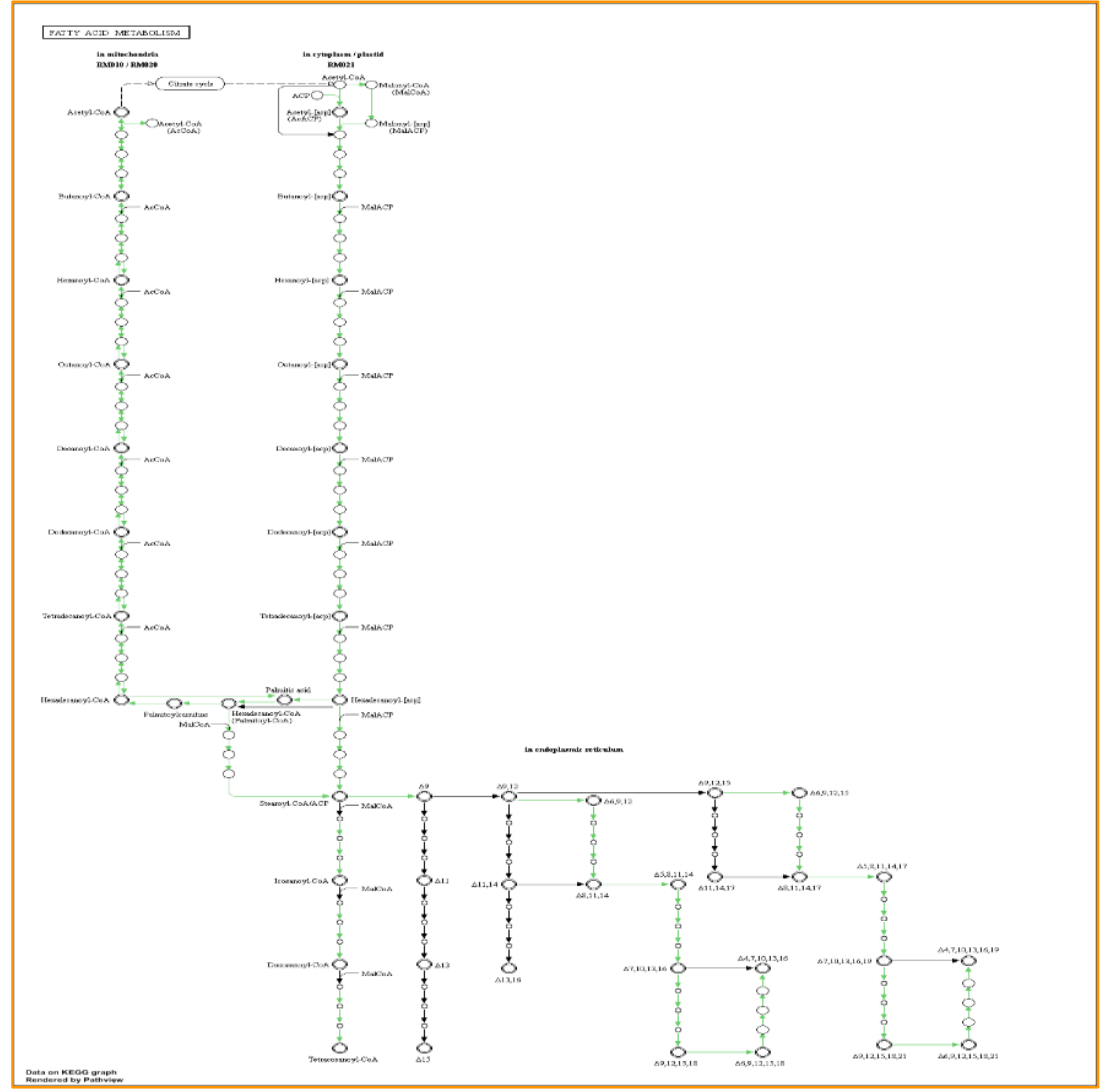

Figure 36: Pathview of the "fatty acid metabolism" KEGG pathway in wild-type and STAT1F77A mice following LAD ligation. 

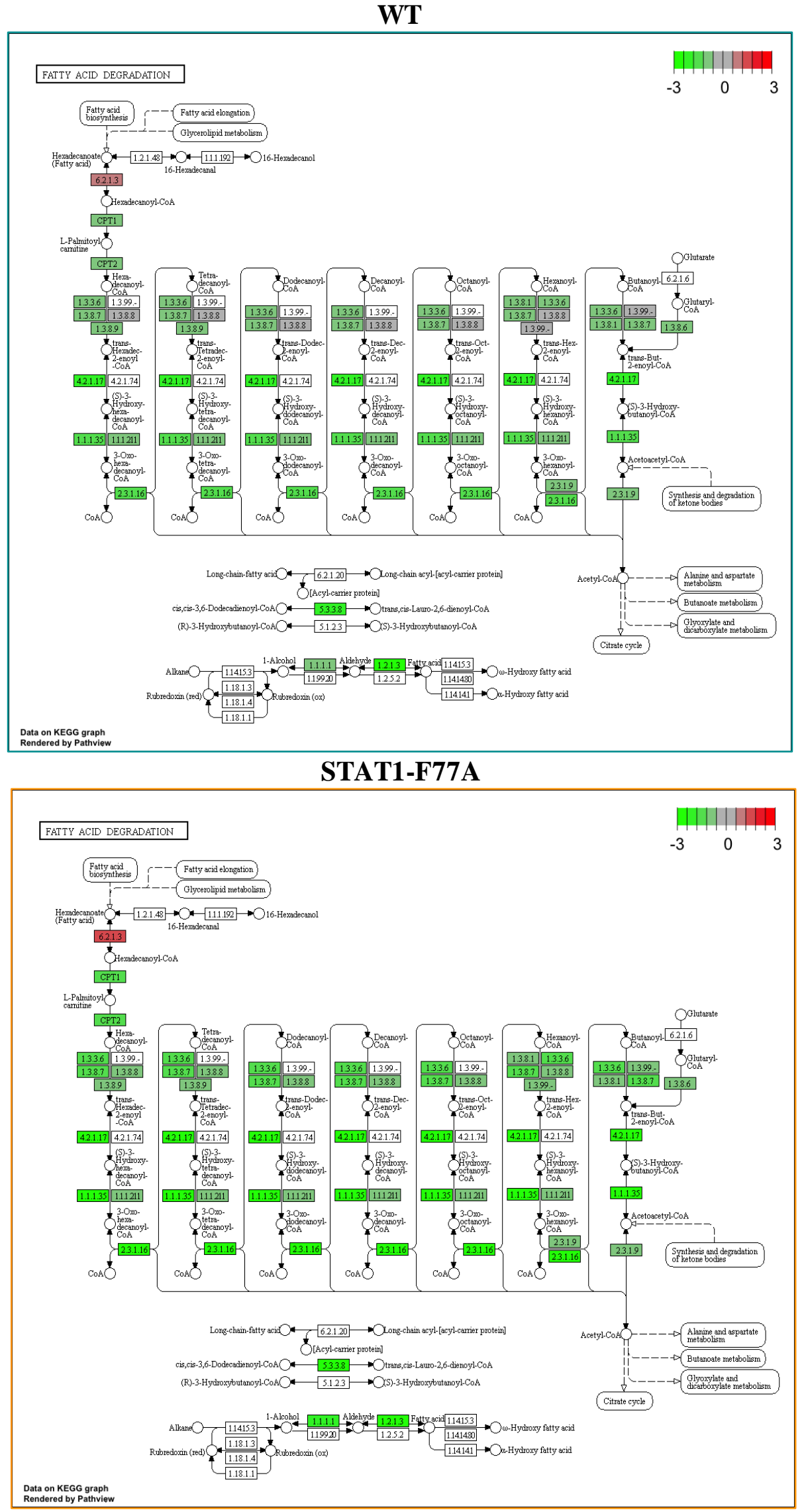

Figure 37: Pathview of the "fatty acid degradation" KEGG pathway in wild-type and STAT1F77A mice following LAD ligation. 


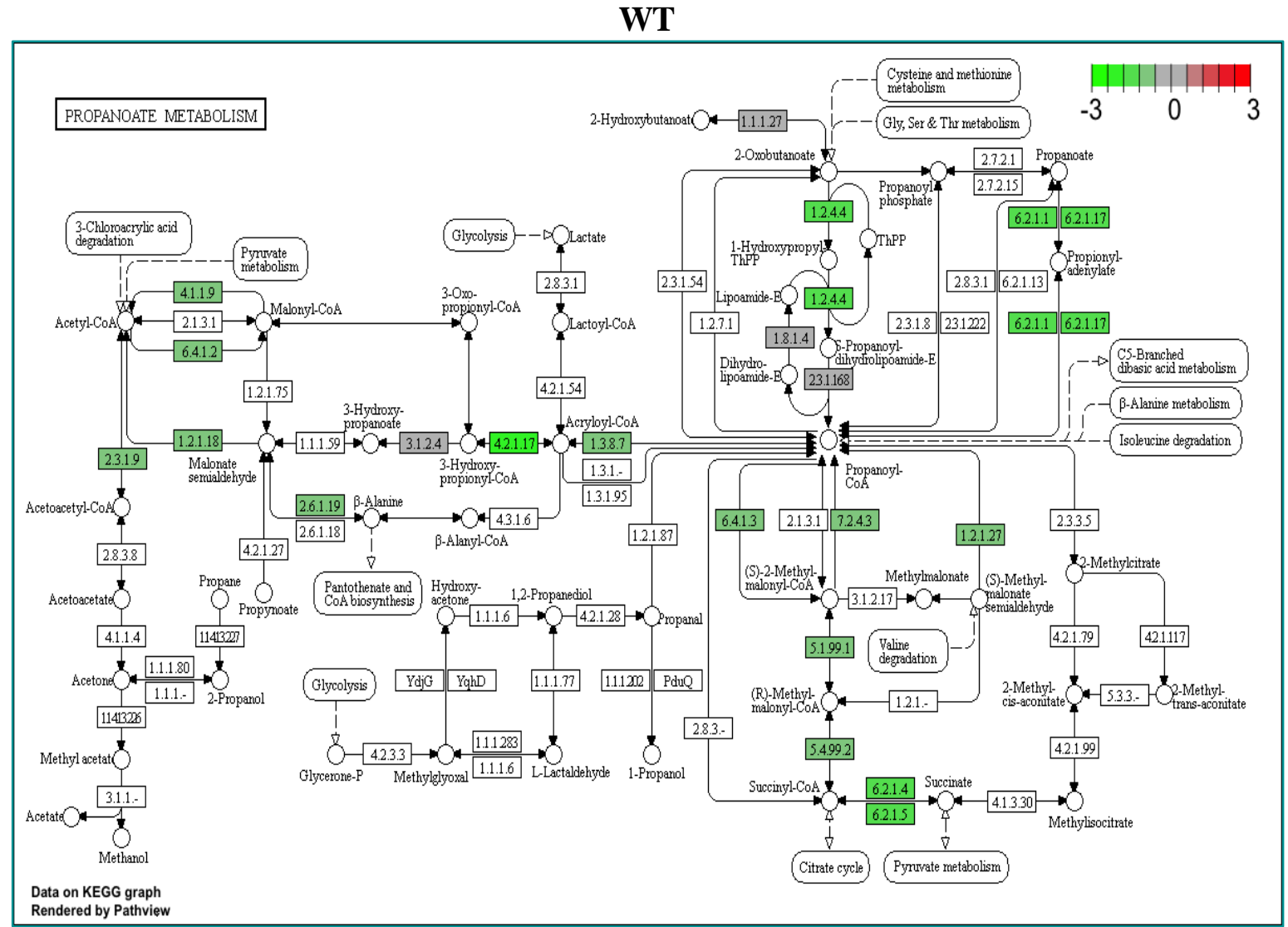

Rendered by Pathview

STAT1-F77A

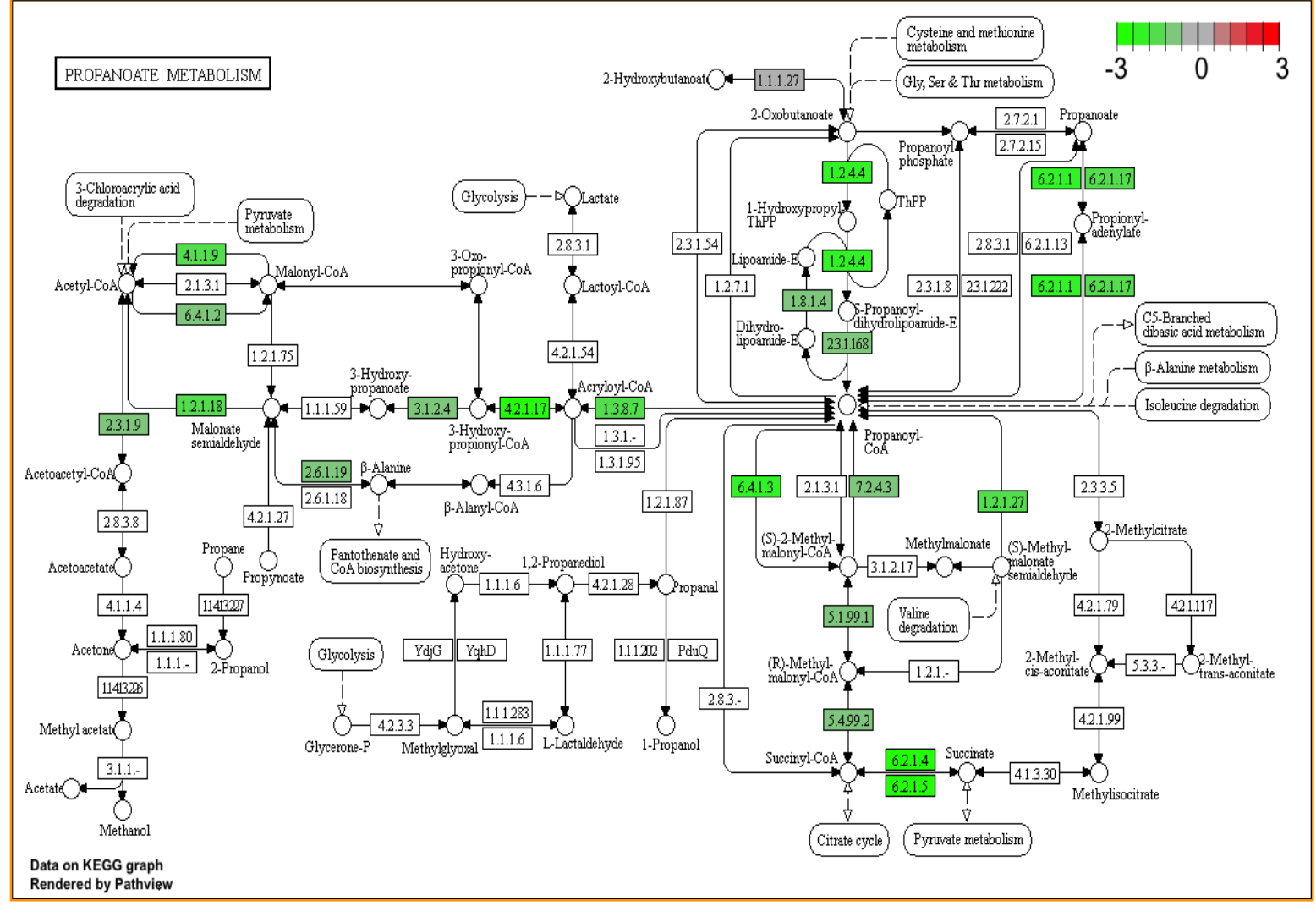

Figure 38: Pathview of the "propanoate metabolism" KEGG pathway in wild-type and STAT1F77A mice following LAD ligation. 
mmu00640:Propanoate metabolism mmu00280:Valine, leucine and isoleucine degradation mmu00650:Butanoate metabolism mmu00920:Sulfur metabolism mmu00071:Fatty acid degradation mmu00450:Selenocompound metabolism mmu00380:Tryptophan metabolism mmu00410:beta-Alanine metabolism mmu00062:Fatty acid elongation mmu01212:Fatty acid metabolism

mmu00120:Primary bile acid biosynthesis mmu00982:Drug metabolism - cytochrome P450mmu04964:Proximal tubule bicarbonate reclamation mmu00480:Glutathione metabolism

mmu01210:2-Oxocarboxylic acid metabolism mmu00620:Pyruvate metabolism mmu00980:Metabolism of xenobiotics by cytochrome P450 mmu03320:PPAR signaling pathway mmu00592:alpha-Linolenic acid metabolism mmu00270:Cysteine and methionine metabolism mmu01200:Carbon metabolism mmu04911:Insulin secretion mmu04976:Bile secretion

mmu04920:Adipocytokine signaling pathway mmu00250:Alanine, aspartate and glutamate metabolism mmu05204:Chemical carcinogenesis mmu05414:Dilated cardiomyopathy mmu04146:Peroxisome mmu04930:Type II diabetes mellitus mmu00350:Tyrosine metabolism -

mmu04261:Adrenergic signaling in cardiomyocytes mmu04260:Cardiac muscle contraction mmu00010:Glycolysis / Gluconeogenesis mmu05412:Arrhythmogenic right ventricular cardiomyopathy (ARVC) mmu04923:Regulation of lipolysis in adipocytes mmu00020:Citrate cycle (TCA cycle) mmu00310:Lysine degradation

mmu04925:Aldosterone synthesis and secretion mmu01130:Biosynthesis of antibiotics mmu04713:Circadian entrainment

mmu00330:Arginine and proline metabolism mmu04024:CAMP signaling pathway mmu04922:Glucagon signaling pathway mmu00591:Linoleic acid metabolism mmu05410:Hypertrophic cardiomyopathy (HCM) mmu04931:Insulin resistance mmu00590:Arachidonic acid metabolism mmu04152:AMPK signaling pathway mmu02010:ABC transporters mmu05032:Morphine addiction mmu04971:Gastric acid secretion mmu04912:GnRH signaling pathway mmu00561:Glycerolipid metabolism mmu04921:Oxytocin signaling pathway mmu01230:Biosynthesis of amino acids mmu00564:Glycerophospholipid metabolism mmu04022:cGMP-PKG signaling pathway mmu04723:Retrograde endocannabinoid signaling mmu04727:GABAergic synapse mmu04020:Calcium signaling pathway mmu04972:Pancreatic secretion mmu04910:Insulin signaling pathway mmu04725:Cholinergic synapse mmu04728:Dopaminergic synapse mmu04270:Vascular smooth muscle contraction mmu00230:Purine metabolism mmu04726:Serotonergic synapse mmu01100:Metabolic pathways mmu04724:Glutamatergic synapse mmu05012:Parkinson's disease
$30 \bigcirc 60 \bigcirc 90$

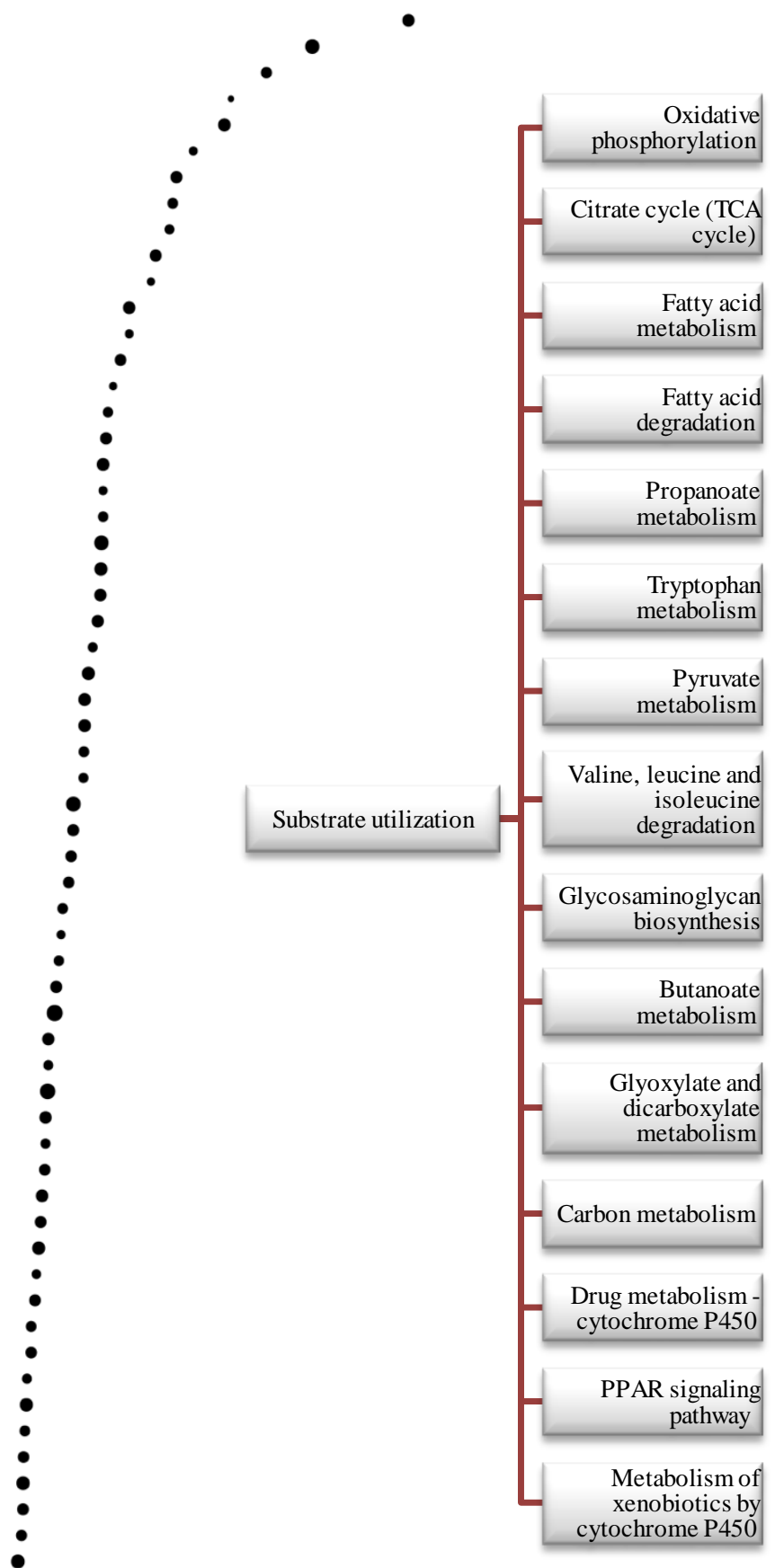




\subsection{Differentially regulated pathways in F77A as compared to wild-type mice in the infracted area following LAD ligation}

Using KEGG pathway enrichment analysis, 18 KEGG pathways were identified when comparing STAT1-F77A and wild-type mice post-LAD ligation, among which oxidative phosphorylation, cardiac muscle contraction, citrate cycle were observed to be the top three in mean enrichment score (Figure 40). Table 31 below illustrates the KEGG pathway ID, KEGG name, mean enrichment score, $p$-value, $q$-value, and the number of genes in each respective pathway comparing STAT1-F77A and wild-type mice post-MI. Furthermore, gene set enrichment analysis revealed differential expression of cardiac muscle contraction pathway only in STAT1-F77A mice at day 1 post-MI (Figure 41).

Table 31: List of KEGG differentially regulated pathways comparing STAT1-F77A and wildtype mice post-MI.

\begin{tabular}{llllll}
\hline KEGG ID & KEGG pathway & mean & $\boldsymbol{p}$-value & $\boldsymbol{q}$-value & size \\
\hline $\mathrm{mmu} 00190$ & Oxidative phosphorylation & -8.2 & $1.48 \mathrm{e}-14$ & $3.26 \mathrm{e}-12$ & 116 \\
\hline $\mathrm{mmu} 04260$ & Cardiac muscle contraction & -4.9 & $1.33 \mathrm{e}-06$ & $1.46 \mathrm{e}-04$ & 67 \\
\hline $\mathrm{mmu} 00020$ & Citrate cycle (TCA cycle) & -4.1 & $7.43 \mathrm{e}-05$ & $5.45 \mathrm{e}-03$ & 30 \\
\hline $\mathrm{mmu} 00280$ & Valine, leucine and isoleucine degradation & -3.8 & $1.36 \mathrm{e}-04$ & $6.53 \mathrm{e}-03$ & 47 \\
\hline $\mathrm{mmu00640}$ & Propanoate metabolism & -3.9 & $1.48 \mathrm{e}-04$ & $6.53 \mathrm{e}-03$ & 29 \\
\hline $\mathrm{mmu} 04621$ & NOD-like receptor signalling pathway & 4 & $3.46 \mathrm{e}-05$ & $7.61 \mathrm{e}-03$ & 146 \\
\hline $\mathrm{mmu04723}$ & Retrograde endocannabinoid signalling & -3.5 & $3.08 \mathrm{e}-04$ & $1.13 \mathrm{e}-02$ & 122 \\
\hline $\mathrm{mmu03008}$ & Ribosome biogenesis in eukaryotes & 3.4 & $5.00 \mathrm{e}-04$ & $3.86 \mathrm{e}-02$ & 72 \\
\hline $\mathrm{mmu04380}$ & Osteoclast differentiation & 3.3 & $5.26 \mathrm{e}-04$ & $3.86 \mathrm{e}-02$ & 122 \\
\hline $\mathrm{mmu00620}$ & Pyruvate metabolism & -3.1 & $1.34 \mathrm{e}-03$ & $4.22 \mathrm{e}-02$ & 33 \\
\hline $\mathrm{mmu} 04657$ & IL-17 signalling pathway & 3 & $1.60 \mathrm{e}-03$ & $6.46 \mathrm{e}-02$ & 78 \\
\hline $\mathrm{mmu04144}$ & Endocytosis & 3 & $1.65 \mathrm{e}-03$ & $6.46 \mathrm{e}-02$ & 240 \\
\hline $\mathrm{mmu} 04668$ & TNF signalling pathway & 2.9 & $1.85 \mathrm{e}-03$ & $6.46 \mathrm{e}-02$ & 108 \\
\hline $\mathrm{mmu04060}$ & Cytokine-cytokine receptor interaction & 2.8 & $2.43 \mathrm{e}-03$ & $6.46 \mathrm{e}-02$ & 204 \\
\hline $\mathrm{mmu03013}$ & RNA transport & 2.8 & $2.46 \mathrm{e}-03$ & $6.46 \mathrm{e}-02$ & 150 \\
\hline $\mathrm{mmu04062}$ & Chemokine signalling pathway & 2.8 & $2.64 \mathrm{e}-03$ & $6.46 \mathrm{e}-02$ & 167 \\
\hline $\mathrm{mmu} 01200$ & Carbon metabolism & -2.8 & $3.11 \mathrm{e}-03$ & $8.56 \mathrm{e}-02$ & 106 \\
\hline $\mathrm{mmu04218}$ & Cellular senescence & 2.7 & $4.17 \mathrm{e}-03$ & $9.17 \mathrm{e}-02$ & 155 \\
\hline
\end{tabular}



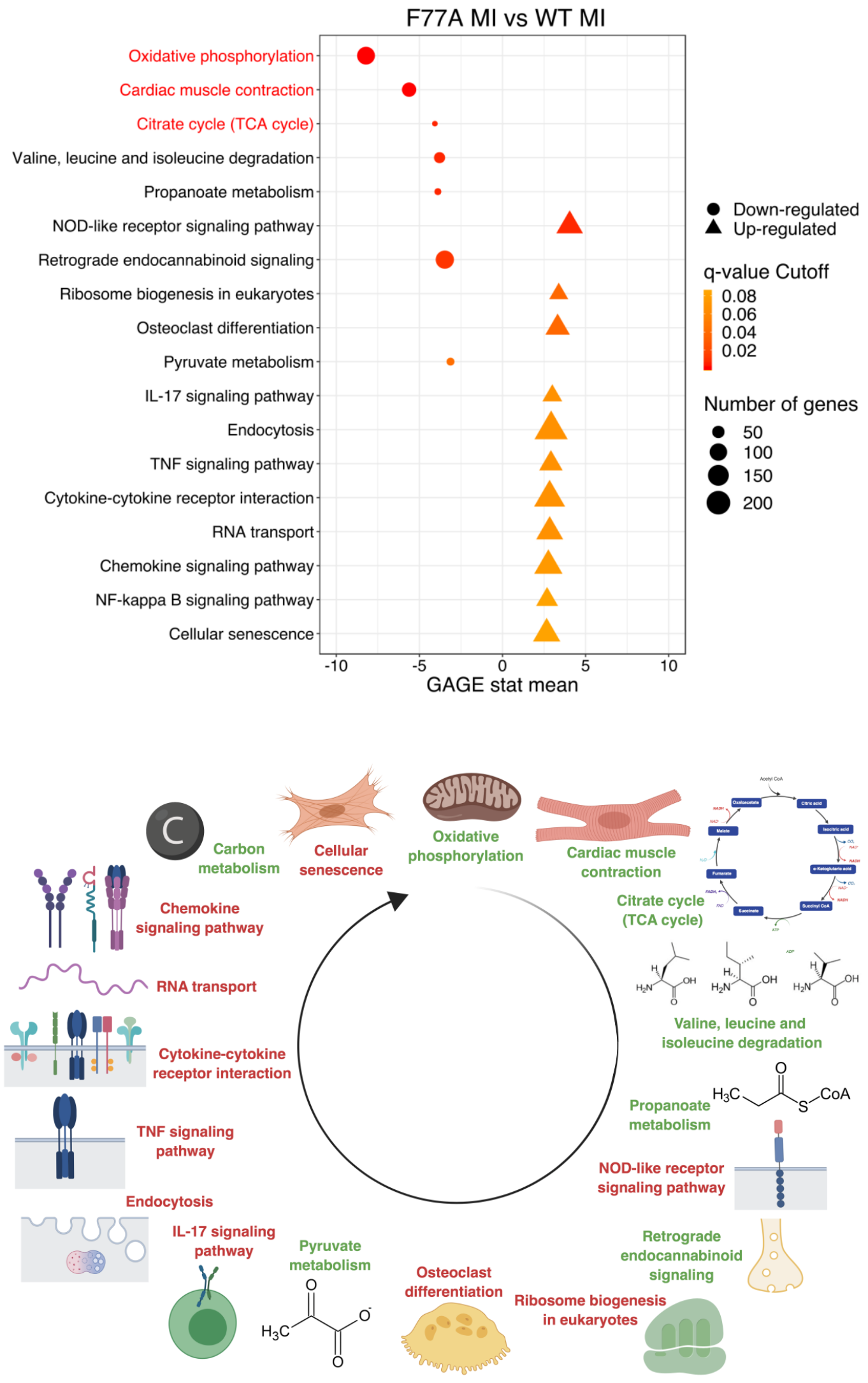

Figure 40: List of KEGG differentially regulated pathways comparing STAT1-F77A MI and wild-type MI mice. GSEA-enriched pathways with q-value $<0.1$ are shown. 




Figure 41: Pathview of the "cardiac muscle contraction" KEGG pathway in STAT1F77A following LAD ligation.

\subsection{A gene expression signature indicative of immune cells infiltration detected in F77A mice following LAD ligation}

Recent studies have demonstrated the prognostic and predictive roles of immune markers in several inflammatory diseases. Nevertheless, additional characterization of these markers in the context of myocardial infarction is evidently warranted. We aimed at identifying putative target genes that are differentially regulated in STAT1-F77A as compared to wild-type. A list of immune markers signifying an immunologically active microenvironment was identified in STAT1-F77A mice with higher cut off value $\left(-\log _{10}(\right.$ padj $\left.)=11\right)$ (Figure 42 and Table 32). 

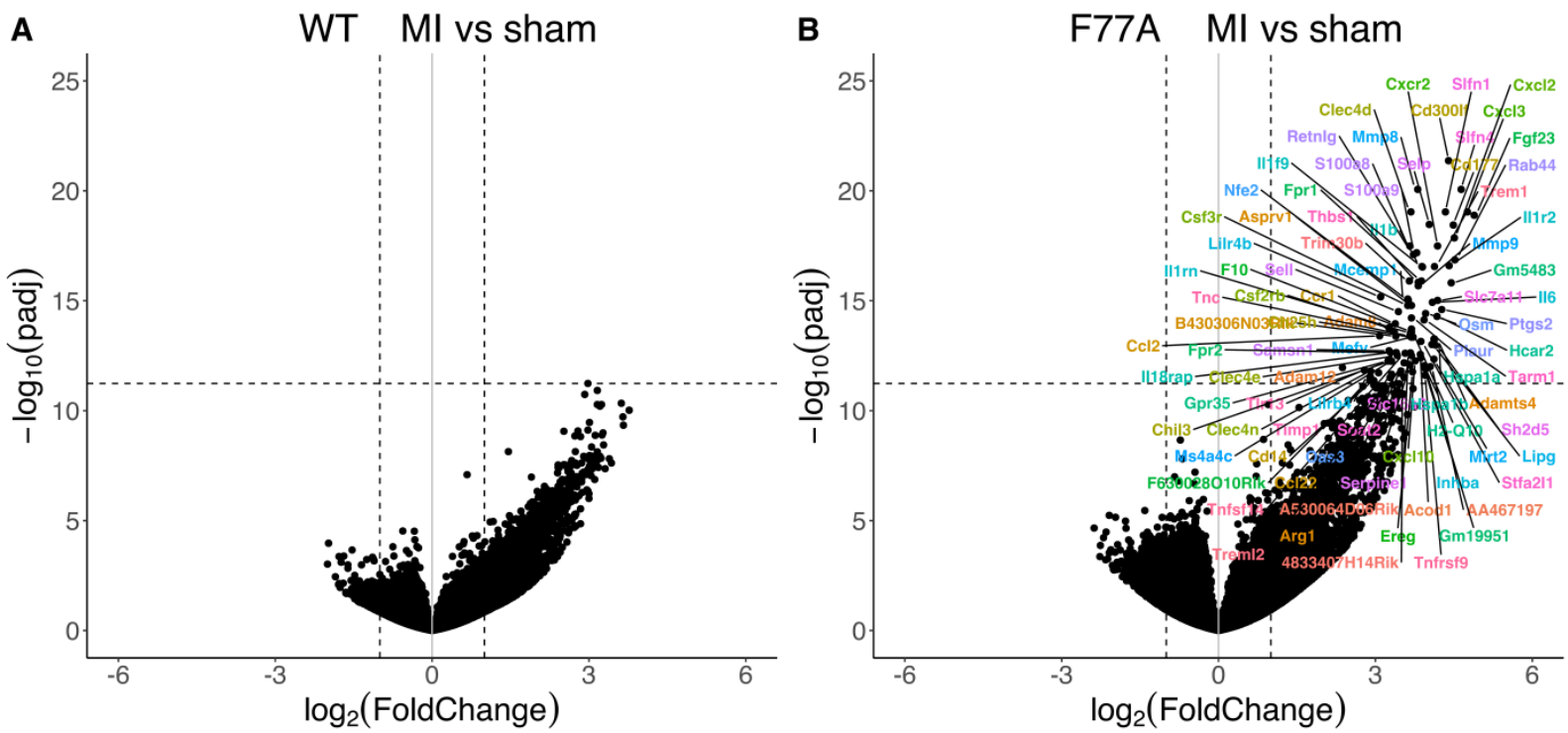

Figure 42: Gene expression signature associated with immune infiltration at day-1 post-MI in STAT1-F77A as compared to wild-type. Each point indicates a gene-of-interest that displays both large magnitude fold-changes ( $x$-axis) as well as high statistical significance (-log 10 of $p$-value, $y$ axis) in STAT1-F77A comparison as compared to wild-type. The horizontal dashed line shows the pvalue cutoff. The vertical dashed lines show 2-fold change cutoff. Therefore, genes that are highly dysregulated are further to the left and right sides, while highly significant changes appear higher on the plot.

These markers can be categorized into immune-activating chemokines and their receptors (e.g. Ccl2, Ccl22, Ccr1, Cxcl10, Cxcl2, Cxcl3, and Cxcr2), adhesion molecules (e.g. S100A8, S100A9, Sell, Selp, Thbs1, Tnc, and Mmp9), cytokines and their receptors (e.g. Cd14, Csf2rb, Csf3r, Fpr1, Il18rap, Ill b, Illf9, Il1r2, Il1 rn, Il6, Inhba, Tnfrsf9, Tnfsf14, Lilrb4, Mefv, Osm), growth factors and their receptors (e.g. Ereg, Fgf23), coagulation cascade proteins (e.g. F10), and heat shock proteins (e.g. Hspala, Hspalb). These data suggest a significant upregulation of both CC and CXC chemokine transcripts in the early period of acute MI, which can play a critical role in repair processes and as a hallmark of the inflammatory response following LAD ligation. Higher expression levels of these chemokines and their receptors and consequently chemokine-receptor interactions may affect immune cell activation and trafficking in the infarcted area of STAT1-F77A mice. The magnitude of remodeling is affected by the chemokine signals involved in the reparative process and the sequential recruitment of immune cell subpopulations that are pro-inflammatory or regulatory to the infarct area. 
Table 32: Genes associated with immune cells activation at day 1 following LAD ligation in STAT1-F77A

\begin{tabular}{|c|c|c|c|}
\hline Gene & $\log _{2}$ fold change & $\log _{10}$ padj & KEGG pathway \\
\hline $\mathrm{CCl} 2$ & 3.08 & $1.34 \mathrm{e}+01$ & Chemokine signalling pathway \\
\hline $\mathrm{Cc} / 22$ & 3.31 & $1.17 e+01$ & Chemokine signalling pathway \\
\hline Ccrl & 3.38 & $1.40 \mathrm{e}+01$ & Chemokine signalling pathway \\
\hline Cd14 & 2.97 & $1.14 \mathrm{e}+01$ & MAPK signalling pathway \\
\hline Csf2rb & 3.26 & $1.38 e+01$ & Jak-STAT signalling pathway \\
\hline Csf3r & 3.62 & $1.48 e+01$ & PI3K-Akt signalling pathway \\
\hline Cxcl10 & 3.76 & $1.23 e+01$ & RIG-I-like receptor signalling pathway \\
\hline$C x C / 2$ & 4.51 & $1.79 e+01$ & Chemokine signalling pathway \\
\hline$C x C / 3$ & 4.76 & $1.90 \mathrm{e}+01$ & Chemokine signalling pathway \\
\hline Cxcr2 & 4.19 & $1.75 e+01$ & Endocytosis \\
\hline Ereg & 3.71 & $1.18 e+01$ & PI3K-Akt signalling pathway \\
\hline$F 10$ & 3.69 & $1.36 \mathrm{e}+01$ & Complement and coagulation cascades \\
\hline Fgf23 & 4.89 & $1.89 e+01$ & Ras signalling pathway \\
\hline Fpr1 & 3.88 & $1.59 e+01$ & Rap1 signalling pathway \\
\hline$H 2-Q 10$ & 3.69 & $1.37 e+01$ & Endocytosis \\
\hline Hspala & 4.12 & $1.33 e+01$ & Protein processing in endoplasmic reticulum \\
\hline Hspa1b & 3.94 & $1.20 \mathrm{e}+01$ & Protein processing in endoplasmic reticulum \\
\hline I/18rap & 3.29 & $1.26 e+01$ & Cytokine-cytokine receptor interaction \\
\hline$I / 1 b$ & 3.89 & $1.66 \mathrm{e}+01$ & Necroptosis \\
\hline I/Ifg & 3.90 & $1.65 e+01$ & Cytokine-cytokine receptor interaction \\
\hline I/1r2 & 4.41 & $1.66 e+01$ & Hematopoietic cell lineage \\
\hline I/Irn & 3.67 & $1.35 e+01$ & Cytokine-cytokine receptor interaction \\
\hline I/6 & 4.09 & $1.49 \mathrm{e}+01$ & HIF-1 signalling pathway \\
\hline Inhba & 3.87 & $1.24 e+01$ & TGF- $\beta$ signalling pathway \\
\hline Lilrb4 & 2.90 & $1.16 e+01$ & Osteoclast differentiation \\
\hline Mefv & 3.72 & $1.33 e+01$ & NOD-like receptor signalling pathway \\
\hline Mmpg & 3.82 & $1.57 e+01$ & Leukocyte transendothelial migration \\
\hline Oas3 & 3.55 & $1.22 \mathrm{e}+01$ & NOD-like receptor signalling pathway \\
\hline Osm & 3.96 & $1.44 \mathrm{e}+01$ & PI3K-Akt signalling pathway \\
\hline Plaur & 3.69 & $1.42 \mathrm{e}+01$ & Complement and coagulation cascades \\
\hline Ptgs2 & 4.27 & $1.46 \mathrm{e}+01$ & NF- $\kappa B$ signalling pathway \\
\hline $5100 a 8$ & 3.66 & $1.75 \mathrm{e}+01$ & IL-17 signalling pathway \\
\hline S100a9 & 3.79 & $1.72 \mathrm{e}+01$ & IL-17 signalling pathway \\
\hline Sell & 3.44 & $1.45 e+01$ & Cell adhesion molecules (CAMs) \\
\hline Selp & 4.03 & $1.85 e+01$ & Cell adhesion molecules (CAMs) \\
\hline
\end{tabular}




\begin{tabular}{llll}
\hline Serpine1 & 3.66 & $1.21 \mathrm{e}+01$ & p53 signalling pathway \\
\hline Thbs1 & 3.82 & $1.58 \mathrm{e}+01$ & Focal adhesion \\
\hline Timp1 & 2.89 & $1.14 \mathrm{e}+01$ & HIF-1 signalling pathway \\
\hline Tnc & 3.66 & $1.34 \mathrm{e}+01$ & Focal adhesion \\
\hline Tnfrsf9 & 3.95 & $1.16 \mathrm{e}+01$ & Cytokine-cytokine receptor interaction \\
\hline Tnfsf14 & 3.57 & $1.26 \mathrm{e}+01$ & NF- $\kappa$ B signalling pathway \\
\hline
\end{tabular}

padj: Benjamini-Hochberg adjusted $p$-value 


\subsection{Analysis of STAT1-target genes associated with myocardial infarction}

Since a perturbation in STAT1-target gene networks is linked to multiple autoimmune diseases and tumorigenesis, it is noteworthy to investigate the global transcriptional network of STAT1-target genes after myocardial infarction (Cao et al., 2017). Using a list of 1,441 ChIP-Seq-based STAT1 target genes in IFN- $\gamma$-treated HeLa cells published by Satoh and Tabunoki (2013), we constructed a functional protein association network of 132 STAT1target genes that are differentially regulated in wild-type mice (Figure 43) and another protein association network of 180 STAT1-target genes differentially regulated in STAT1-F77A (Figure 44) at day 1 following myocardial infarction. A fold change $>1$ was used as a cut-off value.

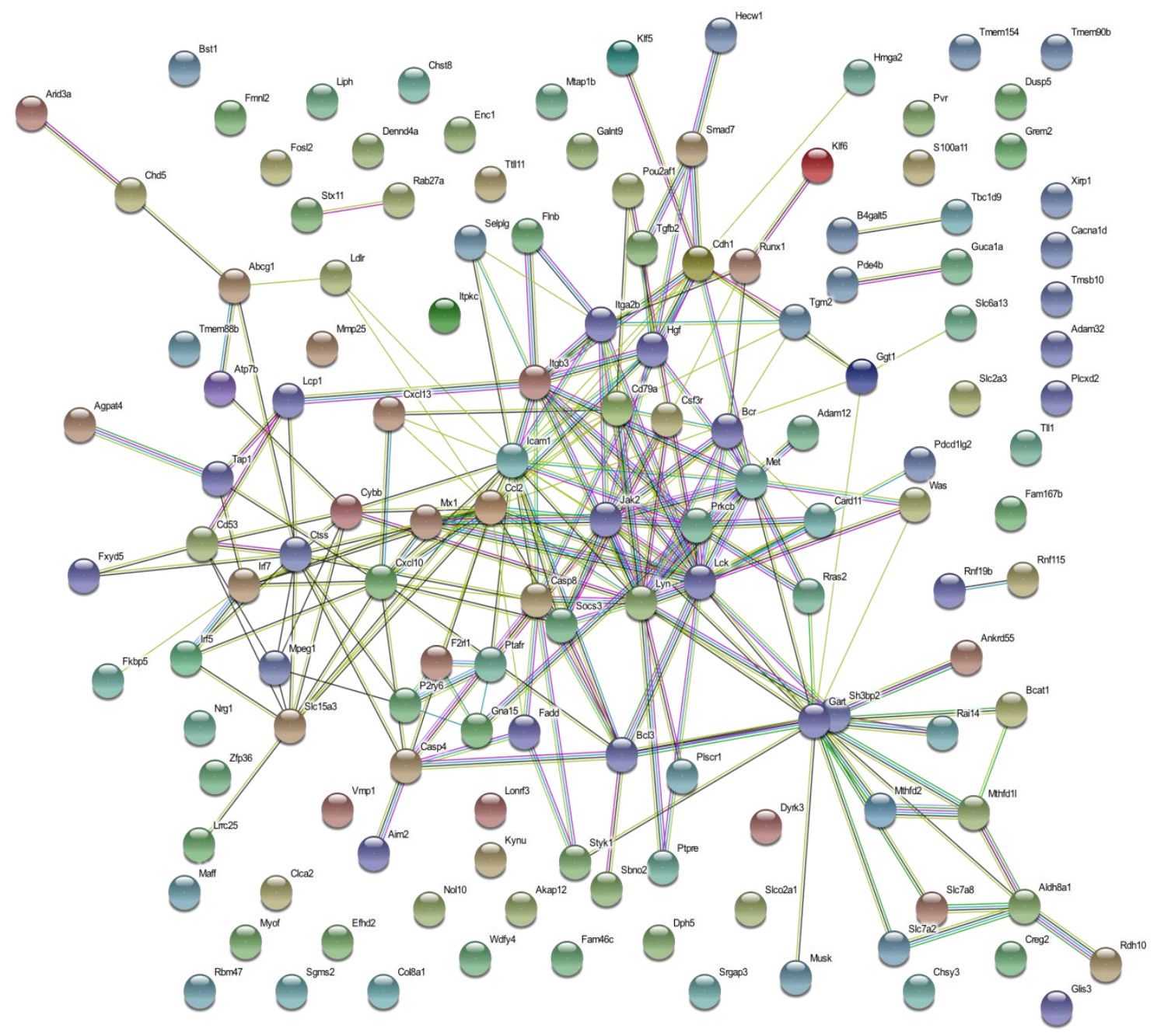

Figure 43: A functional protein association network of 132 STAT1 target genes differentially regulated in wild-type mice post-MI. 


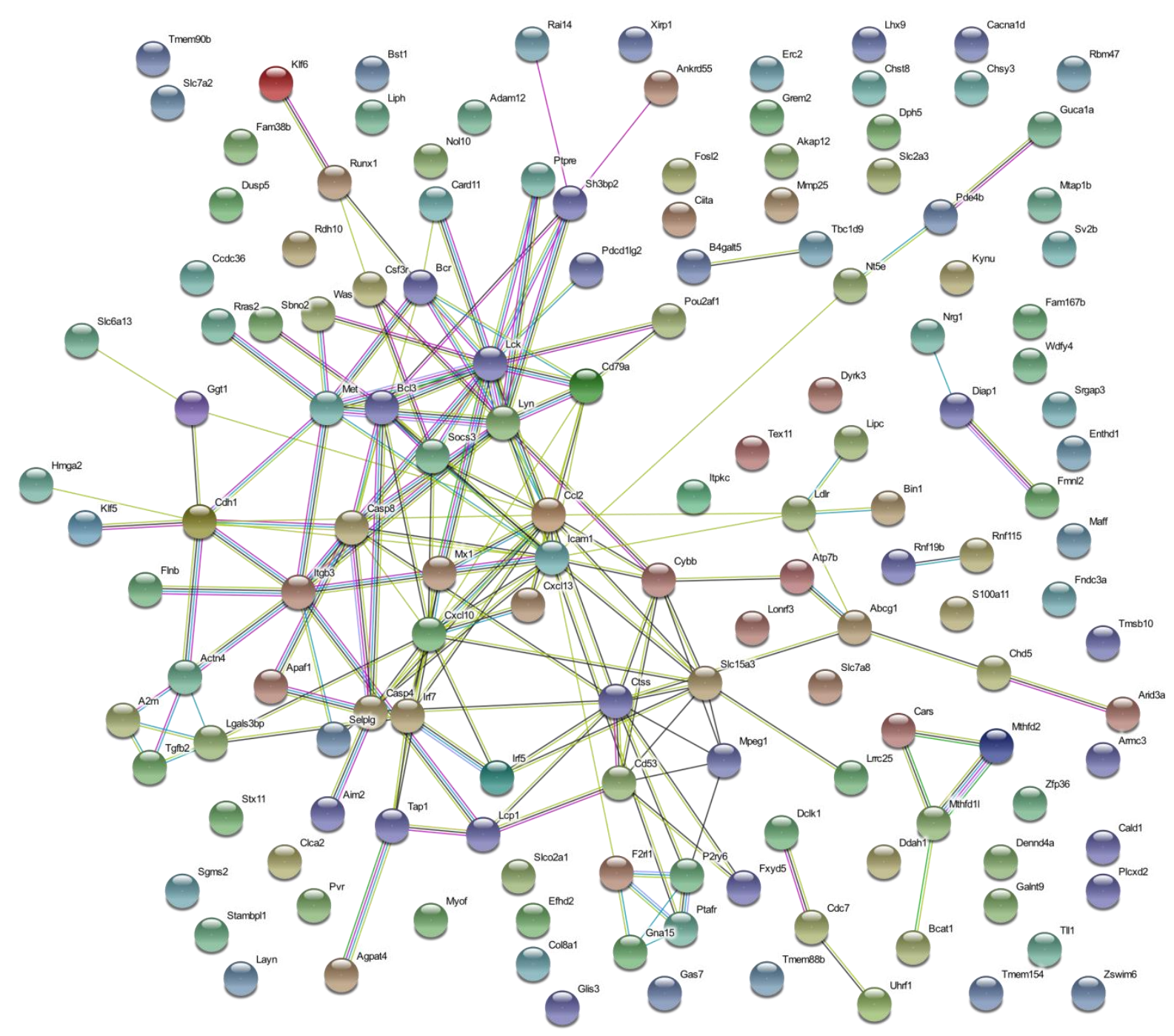

Figure 44: A functional protein association network of 180 STAT1 target genes differentially regulated in F77A post-MI.

\subsection{Co-stimulatory effects of interferon- $\gamma$, interferon- $\alpha$, and interleukin-6 in MHEC-5 cells}

Intriguingly, STAT1-target genes appear to promote inflammation and antagonize proliferation. This contrasts the pro-proliferative and anti-inflammatory activities associated with STAT3, which raises the possibility of balancing the activation of both STAT1 and STAT3 by members of the IFN and IL-6 families. To identify the transcriptional outcome that is induced by activated STAT proteins, we used a murine myocardium-derived endothelial cell line (MHEC-5) to study the phosphorylation levels and the protein expression levels of STAT1 and STAT3 following $45 \mathrm{~min}$ stimulation with IFN- $\alpha$, IFN- $\gamma$, IL-6, or any combination thereof. We found that co-stimulation with IL- 6 and IFN- $\gamma$ reduced the RNA expression level of STAT1 target genes (Cxcl9, Irf1, Gbp2b, Bcl2l1, Stat1, Nnmt) in MHEC-5 
cells compared to cells treated with IFN- $\gamma$ only (Figure 45A), whereas co-stimulation with IL6 and IFN- $\gamma$ did not alter the level of STAT1 tyrosine phosphorylation in comparison to cells treated with IFN- $\gamma$ only (Figure 45B).

A)
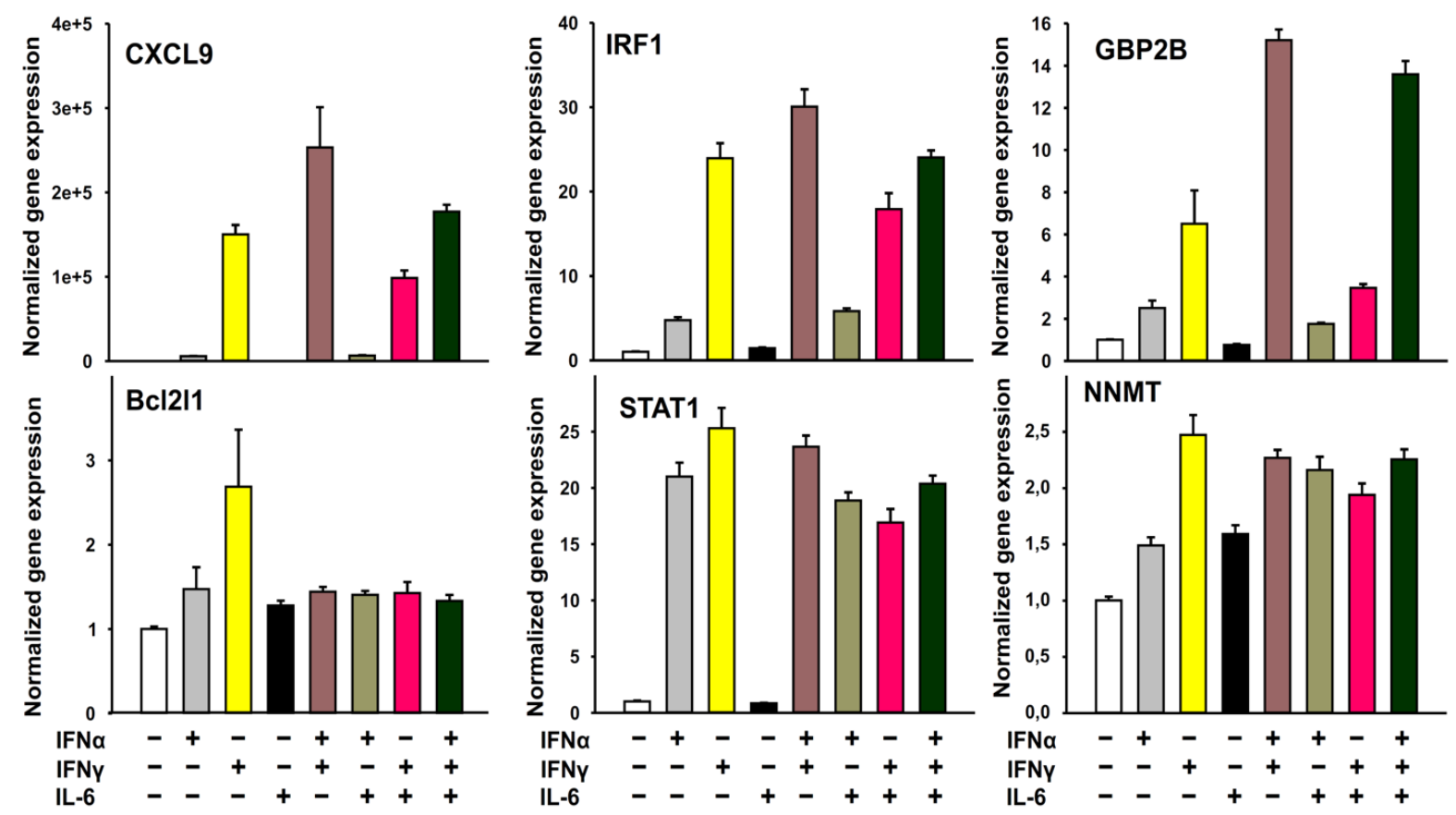

B)

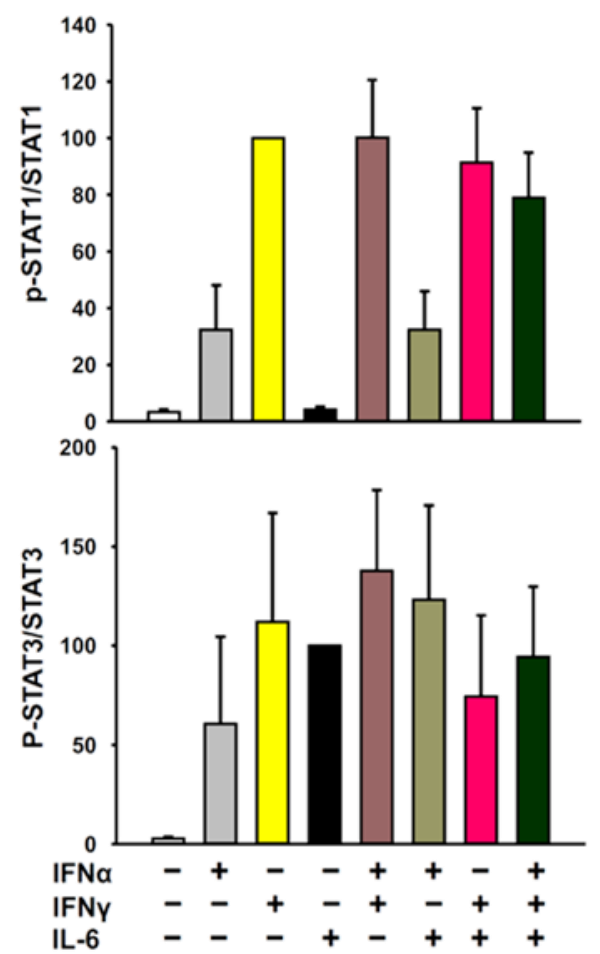

Figure 45: Co-stimulatory effects of interferon- $\gamma$, interferon- $\alpha$, and interleukin-6 in MHEC-5 cells on (A) the induction of STAT1 target gens and (2) the expression and phosphorylation levels of STAT1 and STAT3 proteins 


\subsection{Generation of STAT1- and STAT3-knockdown HL-60 cell lines}

In addition to acting as cytoskeletal proteins, the ezrin, radixin and moesin (ERM) proteins act as signaling adaptors and play a fundamental role regulating invasion and cellular cortical tension, both of which are required for efficient transendothelial migration. Given the complex phenotype presented in the STAT1-F77A mice challenged with MI, STAT1 and STAT3 were systematically knocked down as a proof of concept study to determine if reducing STAT1 expression in neutrophils would interfere with recruitment and transendothelial migration. Lentiviral vectors with short hairpin RNA (shRNA) were used to transfect the human leukemia cell line HL-60. Immunoblotting was utilized to assess the relative protein levels in STAT1 and STAT3 shRNA-transfected cells compared to cells transfected with control shRNA. Total STAT1 levels were reduced following transfection with 3 different anti-STAT1 shRNA, whereas total STAT3 levels were reduced using 5 different anti-STAT3 shRNA viral clones, as can be seen in Figure 46. HL-60 cells that are transfected with lentiviral shSTAT1 clone\#3 and HL-60 cells transfected with shSTA3 clone\#5 were used in the following experiments, as they have shown the highest percentage of knockdown according to the ratios of STAT1 and STAT3 proteins to $\beta$-actin in each lane in comparison to shRNA control.

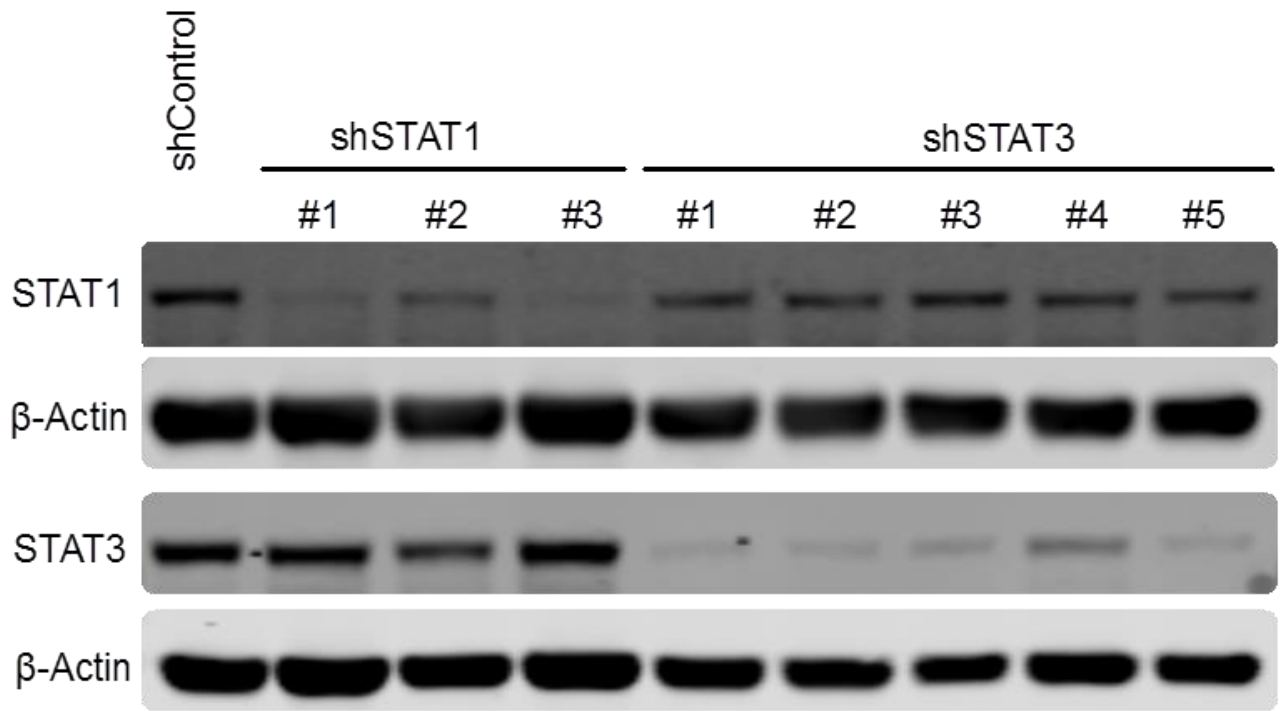

Figure 46: shRNA knockdown of STAT1 and STAT3 in HL60 untreated cells 


\subsection{DMSO-induced differentiation results in upregulation of STAT1 and STAT3 in}

\section{HL-60 cells}

In order to further characterize the mechanisms involved in HL-60 cells differentiation, we examined the kinetics of STAT1 and STAT3 expression in response to $1.3 \%$ DMSO stimulation. Protein extracts were collected at days $0,1,2,3,4$, and 5 and were subjected to immunoblot analyses. DMSO stimulation led to the induction of STAT1 and STAT3 expression, where the peak of STAT1 expression was at day 4, and the peak of STAT3 expression was at day 5 in STAT1 and STAT3-knockdown cells, respectively (Figure 47). These data suggest that STATs signalling is involved in the differentiation of HL-60 cells.

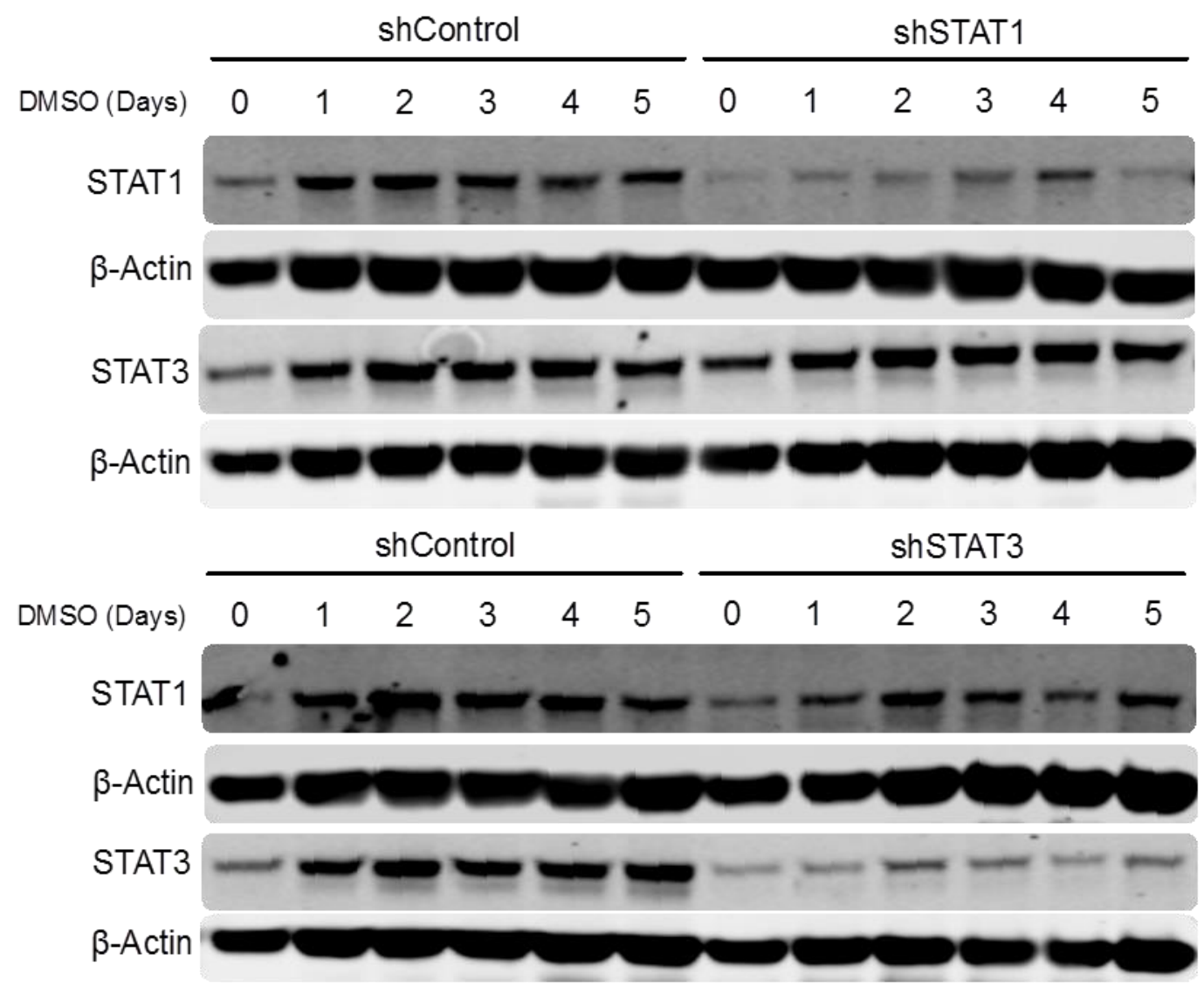

Figure 47: STAT1 and STAT3 expression levels in control as well as STAT1- and STAT3knockdown HL-60 cells differentiated with DMSO

\subsection{Decreased phosphorylation levels of STAT1 and STAT3 in HL-60 knockdown cells}

To further characterize the generated STAT1- and STAT3-knockdown cells, we differentiated HL-60 cells with $1.3 \%$ DMSO and analyzed them at day 4 for the phosphorylation levels of STAT1 and STAT3. Cells were left unstimulated or stimulated with IFN- $\gamma$ (50 ng/ml), IL-6 
(10 ng/ml) or both IFN- $\gamma$ and IL-6 for 6 and 24 hours. Our results show that STAT1 phosphorylation levels upon stimulation with IFN- $\gamma$ or both IFN- $\gamma$ and IL-6 are significantly increased, whereas STAT3 phosphorylation is induced in IFN- $\gamma$, IL-6 or both IFN- $\gamma$ and IL-6 stimulation conditions as compared to unstimulated cells. Additionally, STAT1 and STAT3 phosphorylation levels are significantly decreased in STAT1-knockdown and STAT3knockdown HL-60 cells, respectively, as compared to control shRNA transfected cells. Moreover, immunoblots of STAT1 and STAT3 demonstrated that downregulation of STAT1 in HL-60 cells by shRNA expression had no effect on STAT3 expression level. However, the knockdown of STAT3 in HL-60 cells by shRNA had a negative effect on the expression levels of STAT1. Furthermore, the phosphorylation levels of STAT1 were reduced in STAT3-knockdown HL-60 cells as compared to control shRNA (Figure 48 and Figure 49).

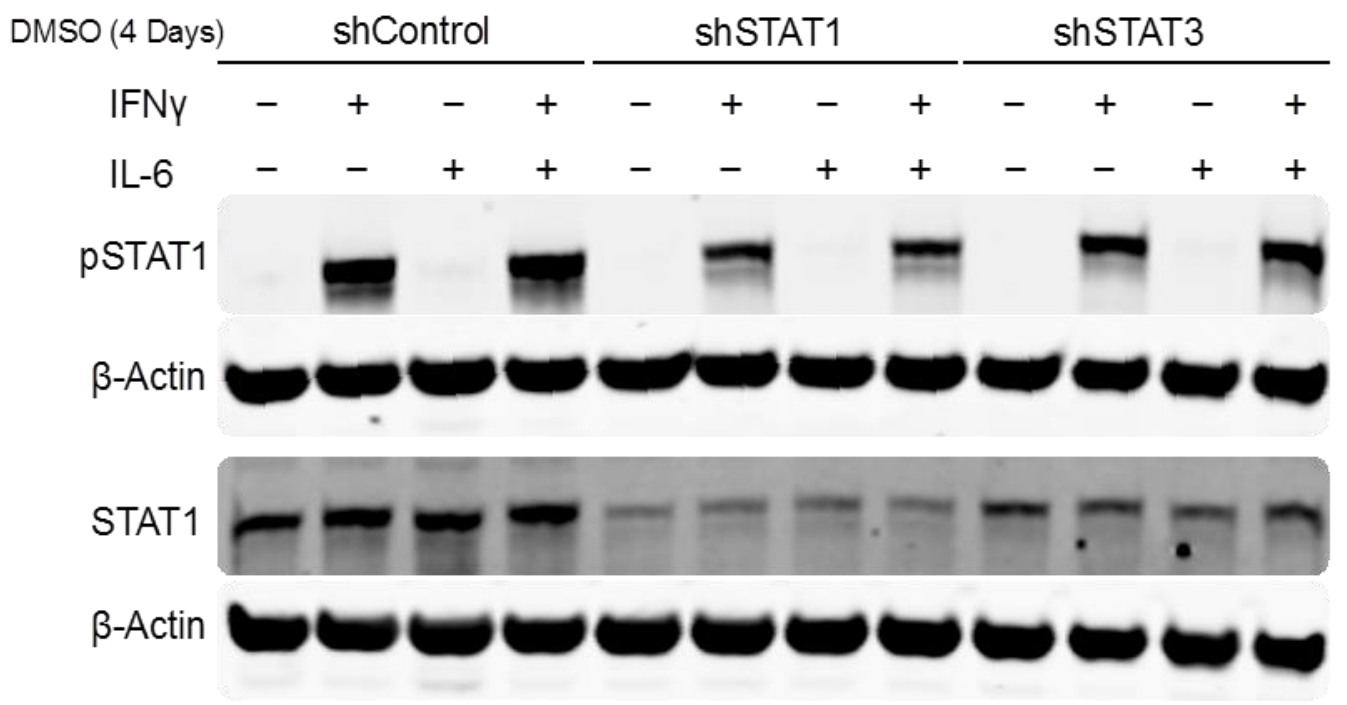

Figure 48: STAT1 expression levels and phosphorylation in HL-60 cells differentiated with DMSO and stimulated at day 4 with IFN- $\gamma$, IL-6 or the combination of both for 45 min. 


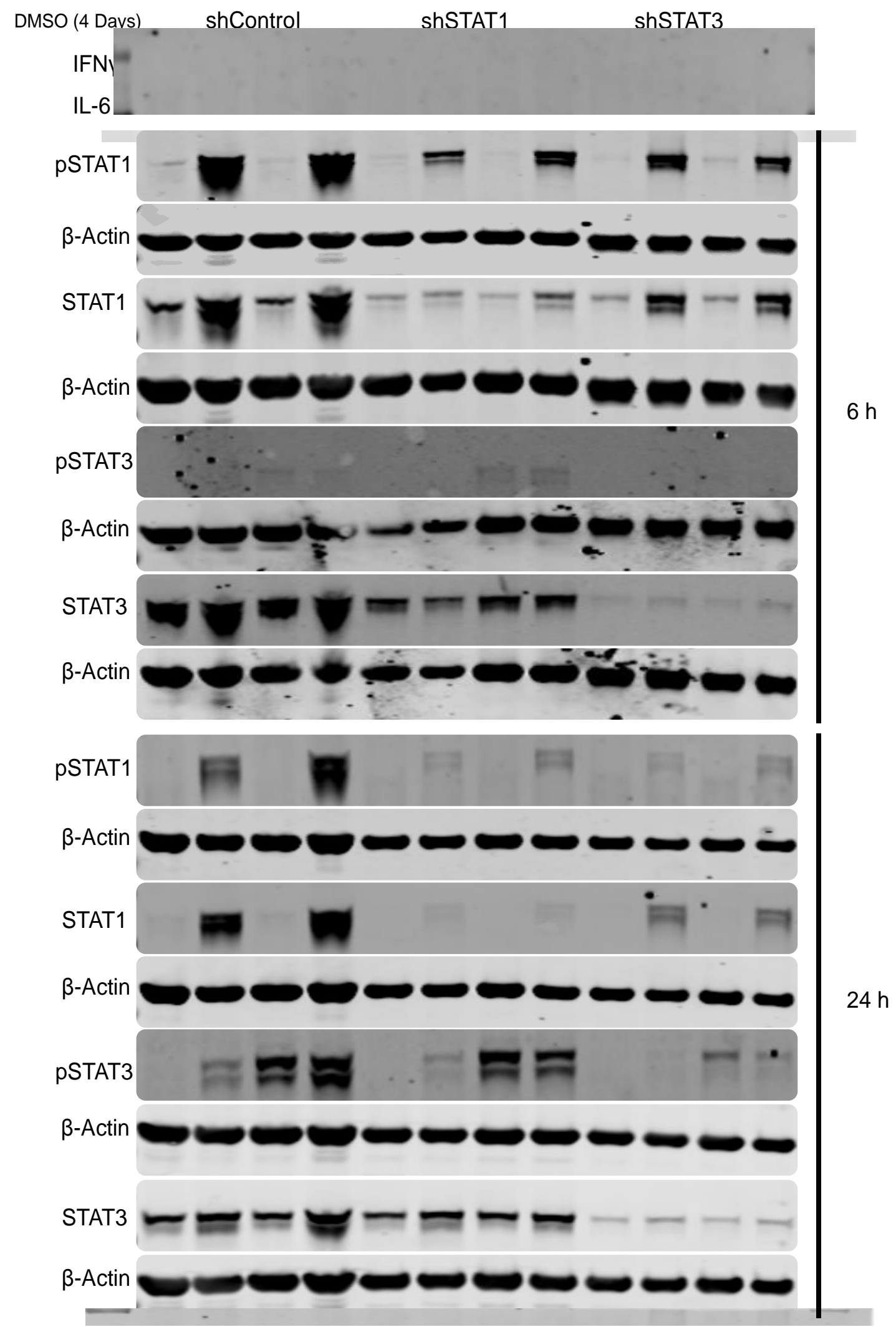

Figure 49: STAT1 and STAT3 expression and phosphorylation in control, shSTAT1, and shSTAT3 HL-60 cells differentiated with DMSO. Stimulation was performed with IFN- $\gamma, I L-6$ or combination of both for $6 h, 24 h$ at analyzed at day 4 


\subsection{Downregulation of ezrin in STAT1- and STAT3-knockdown HL-60 cells}

Ezrin, a member of the ezrin/radixin/moesin (ERM) family of proteins, cross-links the cytoskeleton and the cell membrane mediating various cellular processes. Here, to investigate the expression of ezrin, we blotted for ezrin in control and STAT1 and STAT3-knockdown HL-60 cells that are differentiated with DMSO (day 4). In this preliminary data we found that the knockdown of STAT1 and STAT3 by shRNA decreased the expression of ezrin in HL-60 cells when left untreated or treated with IFN- $\gamma$ and IL-6 or a combination of both for 24 hours compared to control shRNA cells (Figure 50).

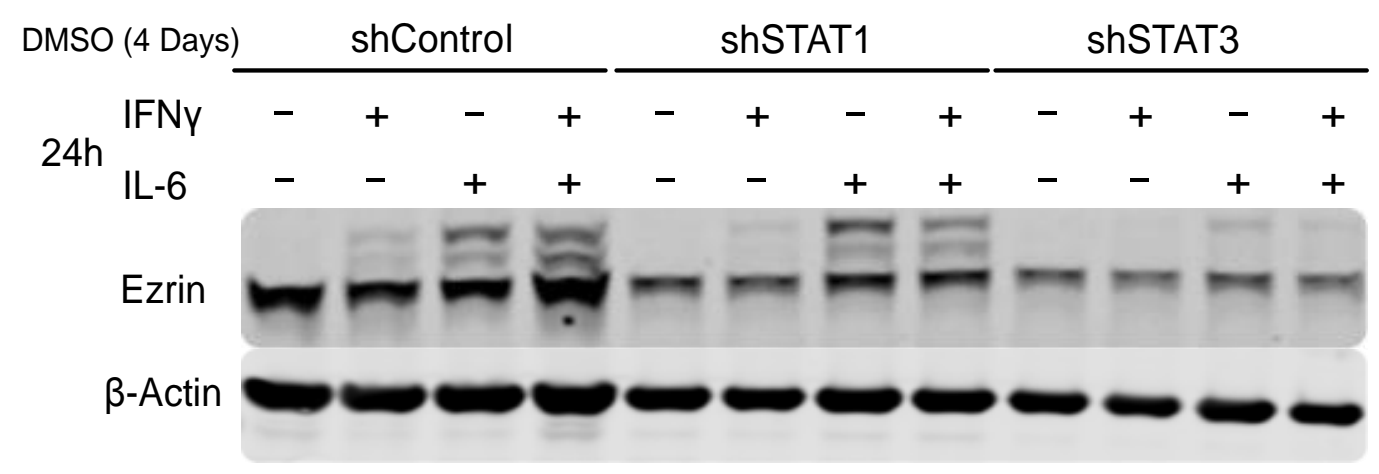

Figure 50: Ezrin expression levels in control, STAT1- and STAT3-knockdown HL-60 cells differentiated with 1.3\% DMSO. Cells were analyzed at day 4 and left unstimulated or stimulated with IFN- $\gamma$, IL-6 or combination of both for 24 hours.

\subsection{Reduced transendothelial migration in HL-60 cells with decreased STAT1 and STAT3 expression}

Parallel plate flow chamber assays were used for live-cell imaging to investigate the role of STAT1 and STAT3 on the recruitment and transmigration of neutrophil-like HL-60 cells to activated endothelial monolayers under constant flow conditions. While tracking of transmigrating cells have shown a reduced transendothelial migration in both STAT1 and STAT3-knockdown HL-60 cells, STAT3-knockdown resulted in a more pronounced inhibition of transmigration across the HUVEC endothelial monolayer as compared to control cells $(n=3)$ (Figure 51). Interestingly, STAT3 knockdown has further impacted cell polarity and directional persistence in HL-60 cells. 

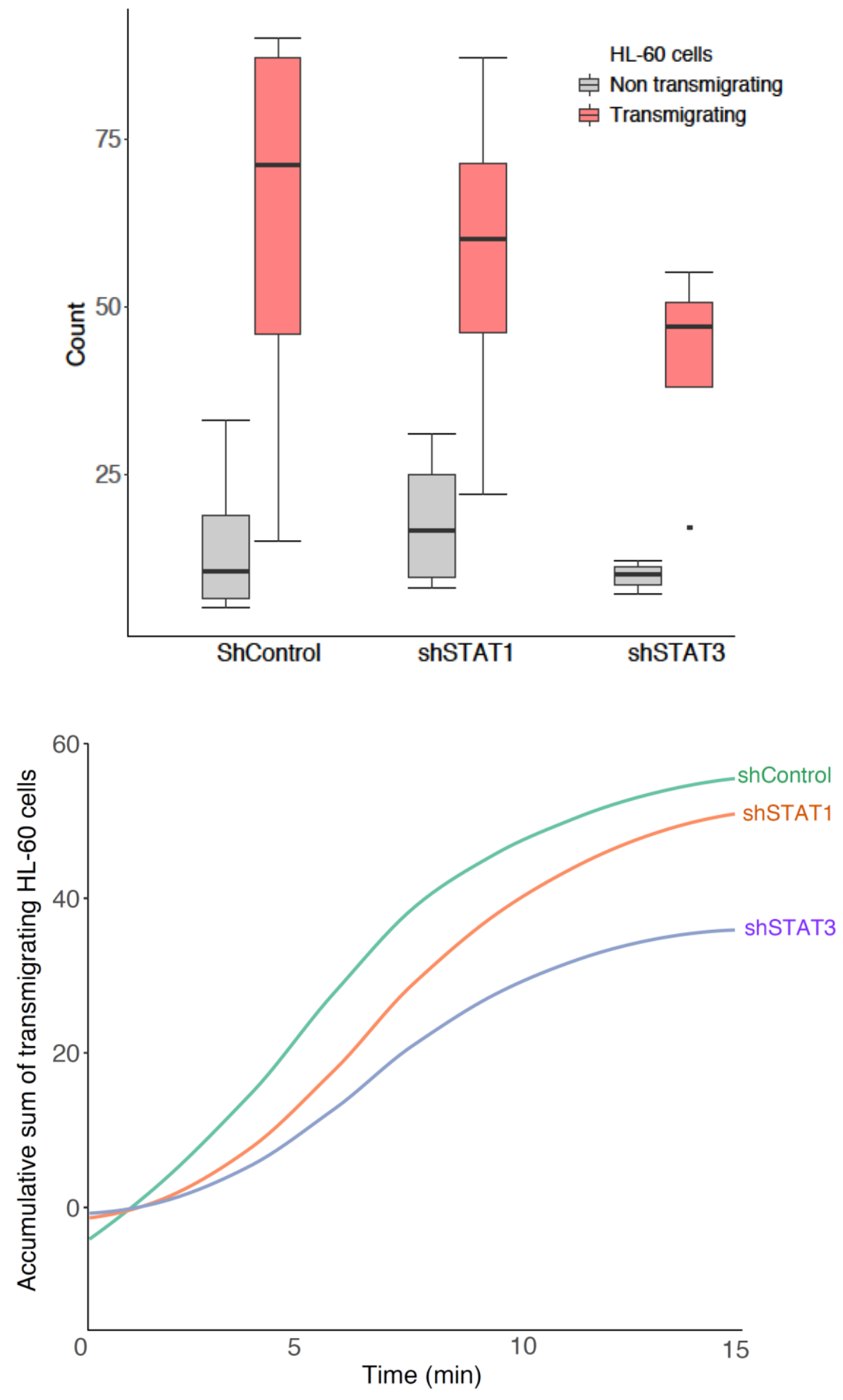

Figure 51: Extravasation analysis of HL-60 cells across HUVEC monolayer in STAT1 and STAT3 shRNA-transfected cells compared to cells transfected with control shRNA. All cells were differentiated with DMSO and analyzed at day 4. 


\section{Discussion}

The Janus kinase-signal transducer and activator of transcription (JAK-STAT) signalling pathway has been identified as a critical regulator of several physiological responses following cardiac insult including oxidative damage, myocarditis, and cardiac hypertrophy. Moreover, elevated levels of circulating cytokines have been recognized to activate the JAKSTAT signal transduction and modulate the functional status of heart patients (Rumalla et al., 2002; Jug et al., 2009; Parissis et al., 2004; Ruppert and Meyer, 2007; Simon et al., 1998; Ueland et al., 2015; Wagner and Siddiqui, 2012).

Interferon signalling is initiated by binding of the cytokine to the extracellular domain of its cognate receptor. To facilitate differential regulation of type I and type II interferon signalling, interferon- $\alpha$ receptor recruits STAT1 as well as STAT2, while interferon- $\boldsymbol{\gamma}$ receptor exclusively recruits STAT1. The STAT1 homodimer is formed by interactions between the SH2 domains of two phosphorylated STAT1 protomers. The STAT1-F77A point mutant, with alanine substituted for phenylalanine at position 77 , is defective in forming stabilized tetramers on DNA and shows no cooperative DNA binding (Begitt et al., 2014).

In this project, the role of STAT1-cooperative DNA binding, its transcriptional consequences in the infarcted myocardium, and its impact on cardiac function have been investigated in a mouse model of tetramerisation-deficient STAT1 signalling. To this end, permanent occlusion of the left anterior descending coronary artery was performed to experimentally induce infarction in the left ventricle in wild-type and knock-in STAT1-F77A mice, whereas sham-operated mice were used as controls to determine baseline characteristics.

To assess cardiac structure and function following sham operation and LAD ligation, we analyzed cardiac chamber dimensions, fractional shortening, intracardial volumes, the ejection fraction, and wall thickness using echocardiographic ultrasound imaging with two dimensional views of the heart (short and long axis). Subsequently, RNA-seq technology was used to delineate the role of STAT1-cooperative DNA binding by analyzing RNA samples from the infarcted tissue in wild-type and knock-in mice in comparison to sham-operated left ventricular myocardium (Wang et al., 2009).

Assessment of echocardiographic parameters in wild-type and STAT1-F77A mice demonstrated a crucial role of STAT1 signalling, where IFN- $\gamma$-irresponsive female mice lacking cooperative DNA binding were protected from adverse cardiac remodeling (Figure 4). This finding is consistent with previous observations, in which mice deficient in interferon regulatory factor 3 (IRF3) or type I IFN receptor (IFNAR) have an improved survival rate 
following MI as compared to control mice (King et al., 2017). In the same study, single-cell RNA-seq analysis of 4215 isolated leukocytes from infarcted and healthy heart tissues revealed an activation of the IRF3-interferon axis in cardiac macrophages which had driven a fatal response.

Moreover, treatment of mice with an IFNAR-neutralizing antibody post-MI abrogated the interferon response and improved left ventricular size, contractile function, and survival (King et al., 2017). Similar results were also reported in another study, in which STAT1deficient mice had a significantly smaller infarct size compared to wild-type mice. The authors observed an upregulation of autophagy in STAT1-deficient hearts, characterized by light chain 3 (LC3)-I/LC3-II conversion and an increased expression of cellular autophagy markers Atg12 and Beclin 1, suggesting that the absence of functional STAT1 signalling is protective within the context of myocardial infarction by enhancing autophagy (McCormick et al., 2012).

It has been previously reported that STAT1 activates Fas/FasL genes and apoptosis in cardiac myocytes, when subjected to ischemia/reperfusion injury, which was mediated by the phosphorylation of serine residue 727, but not the tyrosine residue 701 of STAT1 (Stephanou et al., 2001). Further in vitro experiments have shown that STAT1 interacts directly with p53 to regulate the expression of pro-apoptotic target genes, such as Bax, Noxa, and Fas. These data provide further support for the role of STAT1 in promoting DNA damage-induced apoptosis (Townsend et al., 2004). Similarly, a previous study by Stephanou and co-workers demonstrated a higher susceptibility to ischemia-induced cell death in STAT1-transfected cells compared to cells transfected with control plasmid. The authors have also reported an activation of STAT1 and caspase-1 processing in myocytes following ischemia/reperfusion injury using an ex vivo model of intact heart (Stephanou et al., 2000). Over the last decade, the literature has further accumulated enough data to convincingly establish a role for the JAK/STAT signalling pathway in ischemia-reperfusion injury and ischemic post-conditioning (Barry et al., 2007; Boengler et al., 2010; Dawn et al., 2004; Goodman et al., 2008; Goodman et al., 2011; Gross et al., 2006; Heusch et al., 2012; Huffman et al., 2008; Kodama et al., 1998; Smith et al., 2010; Xuan et al., 2005). Of particular importance are the experiments by several groups concerning the cardioprotective effects of STAT3 during ischemia/reperfusion injury as well as under pressure overload (Bolli et al., 2011; Butler et al., 2006; Fuglesteg et al., 2008; Heusch et al., 2011; Hilfiker-Kleiner et al., 2004; Jacoby et al., 2003; Kunisada et al., 2000; Oshima et al., 2005; Pedretti and Raddatz, 2011; Smith et al., 2004; Zhuo et al., 2011). 
Numerous studies in this fast-moving field have now convincingly shown that abrupt reduction in blood flow through the coronary artery can expose the cells to hypoxia, resulting in an irreversible cell damage and necrosis (Semenza et al., 2014). Necrosis, characterized by cytoplasmic granulation and cellular swelling, leads to cell membrane rupture and breakdown of organelles, which consequently culminates in the activation of a severe inflammatory response (Kung et al., 2011). However, the mechanisms of how STAT1 cooperative DNA binding plays a role in mediating this MI-associated inflammatory response have remained unexplored.

In this study, next-generation sequencing (RNA-seq) technology was used to analyze the transcriptome of infarcted tissue in STAT1-F77A mice as compared to wild-type littermates. This approach has gained high-resolution data without any previous knowledge about the sequences under exploration. Within the ongoing efforts to construct a comprehensive model which can predict phenotypes from the transcriptomic data, KEGG (Kyoto Encyclopedia of Genes and Genomes) tries to answer different biological questions by integrating the recent understanding of molecular functions and processes at the level of genes, proteins, and pathways (Kanehisa and Goto, 2000).

Interestingly, our gene set enrichment analysis has revealed PI3K-Akt and JAK-STAT signalling pathways as the top enriched signal transduction pathways following myocardial infarction. A previous study has demonstrated that the activation of PI3K (p110 $\alpha$ ) in mice protects against myocardial infarction-induced heart failure (Lin et al., 2010). In our data set, transcriptomic analysis of cardiac tissue at day 1 following myocardial infarction revealed prominent gene expression changes in wild-type mice and STAT1 tetramerisation-deficient mice. We observed a significantly higher number of differentially expressed genes in STAT1F77A mice, since 2337 genes were differentially regulated with a $\mid \log 2$ (fold change) $\mid>1$ and a p-value $<0.05$, including 909 upregulated genes and 1428 downregulated genes compared to wild-type mice (Figure 8).

Utilizing KEGG database, we have identified common and unique differentially expressed pathways in both wild-type and STAT1-F77A animals following LAD ligation. These were enriched in four categories including immune processes, signal transduction, cellular processes, and metabolism (Figure 13 and Figure 14). The top enriched immune processes that are upregulated post-MI in both wild-type and knock-in mice are cytokine-cytokine receptor interactions, chemokine signalling pathways, cell adhesion molecules, regulation of actin cytoskeleton, and leukocyte transendothelial migration. 
These data are in line with prior studies where gene expression data sets of MI patients were analyzed. For instance, Kontou et al. has identified using blood samples of 93 MI patients and 89 healthy controls differentially expressed genes involved in inflammation, wound healing, nuclear pore proteins, and RNA processing and transport (Kontou et al., 2018). In patients with ST-elevation myocardial infarction (STEMI), Muller and colleagues have used customdesigned DNA array to analyze blood samples at the site of occlusion and in the peripheral circulation during primary percutaneous coronary intervention. Validation of identified genes by qPCR has demonstrated an upregulation of chemokines (Ccl2, $\mathrm{Ccll} 8$ and $\mathrm{Cxcll2}$ ), cellextracellular matrix (Fnl, Cdh5 and Sppl), and lipoprotein modification genes (Apoe) in local versus peripheral blood (Muller et al., 2011).

Moreover, current research has shown that low serum levels of CCL2 chemokine $(\leq 100.9 \mathrm{pg} / \mathrm{mL})$ in 803 patients with acute coronary syndrome were significantly associated with fatal and non-fatal myocardial infarction (Leocádio et al., 2019). Using microarray and protein networks to analyze blood samples from 127 acute MI patients, Devaux and coworkers have demonstrated the involvement of vascular endothelial growth factor B (Vegf-b), thrombospondin-1 (Thbs1) and placental growth factor ( $P g f)$ as potential biomarkers (Devaux et al., 2010). In addition, using RNA-seq of MI patients' blood samples, Zhao and colleagues have identified several immune-related genes, including interferon-induced protein with tetratricopeptide repeats 3 (Ifit3), interferon-induced GTP-binding protein (Mxl), and major histocompatibility complex class II DQ $\alpha 1$ (Hla-dqal) as potentially relevant for myocardial infarction (Zhao et al., 2018).

In a recently published study in mice, several pro-inflammatory transcripts have been detected during the first week post-myocardial infarction, including tumor necrosis factor- $\alpha$ (Tnf- $\alpha)$, monocyte chemoattractant protein-1 (Mcp-1), interleukin-6 (Il-6), and interleukin-1 $\beta$ $(I l 1-\beta)$. Administration of an TNF- $\alpha$ inhibitor has been reported to adversely affect cardiac function, suggesting that inflammatory responses play a protective role in the early stage of myocardial infarction (Wang et al., 2018). Furthermore, recent research has shown in mice that depleting neutrophils from the infarcted myocardium at day 1, using a monoclonal antiGr-1 antibody, had resulted in a downregulation of IFN- $\gamma$ and TNF- $\alpha$ mRNA transcripts, higher mortality rates and worsened cardiac function compared to infarcted controls (Finger et al., 2019). Collectively, these observations indicated that IFN- $\gamma$ mediates a sequential chemotactic cellular immune response that regulates survival and cardiac function.

On the other hand, administration of IL-10 has been shown to improve left ventricular function in rats following MI, which was associated with a significant downregulation of pro- 
inflammatory cytokines and chemokine levels including TNF- $\alpha$, IL-6, and MCP-1. This effect was also accompanied by decreased infiltration of macrophages in the myocardium (Jung et al., 2017; Stumpf et al., 2008). Experimental evidence indicates that transcriptional repression of monocyte interleukin-10 (IL-10) is mediated through STAT1 signalling (VanDeusen et al., 2006). Thus, to inhibit the excessive recruitment of monocyte to the infarcted myocardium, Wang and co-workers have developed poly(ethylene glycol) (PEG)-distearoylphosphatidylethanolamine (PEG-DSPE) micelles loaded with a CCR2 (C-C chemokine receptor type 2) antagonist. These micelles were further surface-decorated with an anti-CCR2 antibody for efficient targeting. Both non-targeted and CCR2-targeting micelles exhibited a tendency to improve cardiac function (Wang et al., 2018). These experiments highlight the crucial effects immune cells play in cardiac remodeling post-MI.

Our results from DESeq2 analysis revealed a set of immune-activating cell markers that were differentially expressed at a higher fold change in the early phase post-MI in STAT1-F77A mice as compared to their wild-type littermates (Figure 42). In particular, the expression profile in the myocardium of STAT1-F77A mice following LAD ligation had identified candidate genes involved in chemokines and their receptors, adhesion molecules, cytokines and their receptors, growth factors and their receptors, coagulation cascade proteins, and heat shock proteins. The top ten differentially upregulated genes in the infarcted myocardium of STAT1-F77A mice were Fgf23, Cxcl3, Slfn4, Rab44, Cxcl2, Cd177, Gm5483, Illr2, Cd300lf, and Slfn1.

Moreover, it is noteworthy to highlight that STAT1-F77A mice had a downregulation in the contractile machinery components compared to wild-type mice (Figure 41). This finding might be a result of a higher ratio of infiltrating immune cells to cardiac cells in the infarcted area, which is essential for cardiac repair processes. This is very interesting in light of accumulating research pinpointing to the inflammatory response as a key factor in ventricular remodeling, where it is associated with chemokine signalling as well as recruitment and migration of different immune cell subsets (Azevedo et al., 2016; Frangogiannis, 2014; Liu et al., 2015; Prabhu and Frangogiannis, 2016; Xia and Frangogiannis, 2017).

To further explore the molecular drivers of myocardial infarction that are under the control of STAT1, we performed a gene set enrichment analysis using the results of the DESeq2 differential expression analysis. Our study in the transgenic mouse line adds to previous findings by identifying top downregulated metabolic pathways post-MI, which are oxidative phosphorylation, citrate cycle, fatty acid metabolism, fatty acid degradation, and propanoate metabolism (Nojiri et al., 2006; Stanton et al., 2000; van Bilsen et al., 2004; Xu et al., 2011). 
In the present study, we demonstrated a higher decrease in the expression of a multitude of genes involved in mitochondrial oxidative phosphorylation, citrate cycle, and substrate utilization in the infarcted myocardium in STAT1-F77A mice. These findings are interesting given that STAT1 has been identified as a transcriptional modulator of Warburg effect in the context of tumorigenesis, regulating genes involved in glycolysis, the citrate cycle and mitochondrial oxidative phosphorylation (Pitroda et al., 2009).

It is important to point out that the highest energy yield in the normal heart comes from metabolizing and consuming non-carbohydrate substrates, such as fatty acids. However, under certain pathological conditions, glucose becomes a preferential substrate in producing energy per oxygen consumption. These alterations are driven by an intricate transcriptional regulatory network that feeds into factors including substrate availability and workload (Nagoshi et al., 2011). Modifications in the oxidative phosphorylation machinery as a result of inflammation-induced NF- $\mathrm{B}$ activation culminate in changes in energy requirement to support systolic and diastolic function (Gordon et al., 2011; Remels et al., 2018). Indeed, it has been suggested that shifting substrate utilization from fatty acids to glucose can improve cardiac function and slow the progression of heart failure (Lionetti et al., 2011).

It has recently been shown that macrophages exhibit distinctive transcriptomic profiles during the first week of myocardial infarction. At day 3 post-MI, macrophages displayed an upregulation of genes involved in proliferation, phagocytosis, mitochondrial function and oxidative phosphorylation, further suggesting an important role for metabolic reprogramming in macrophage polarization (Mouton et al., 2018).

In order to evaluate post-infarction remodeling in a swine model of MI, Yang and co-workers have utilized label-free quantitative proteomics and immunohistochemical analyses (Yang et $a l .$, 2017). Subsequent experiments in their model have shown a significant downregulation of proteins involved in energy metabolism, suggesting dysfunctional myocardial energetics and mitochondrial impairment in the peri-infarct border zone. Numerous other mitochondrial processes were also similarly downregulated, including branched-chain amino acid catabolism, $\beta$-oxidation, fatty acid metabolism, Krebs cycle, and the formation of iron-sulfur clusters (Yang et al., 2017). These results are in line with our observation that MI interferes at a global transcriptional level with oxidative phosphorylation. Taken together, these findings clearly support that the imbalance of cardiac substrate metabolism plays a significant role in the pathogenesis of heart failure (Birkenfeld et al., 2019; Noordali et al., 2018).

We have postulated that STAT1-F77A mice will demonstrate a similar phenotype as mice expressing hyperactive STAT3 with respect to cardioprotective actions. Phillips et al. 
suggested that STAT3 is conceivably regulating mitochondrial machinery through transcriptional regulation or indirect signalling pathways (Phillips et al., 2010). However, according to current concepts activated STAT3 enhances mitochondrial oxidative phosphorylation and promotes transformation by oncogenic Ras. The observed downregulation of oxidative phosphorylation in STAT1-F77A mice may possibly be attributed to the dependence of infiltrating immune cells on glycolysis as a major pathway of energy production which is essential for the restoration of homeostasis (Buck et al., 2017).

Our RNA-seq analysis has several limitations as the data indicate the average gene expression pattern using millions of cells which can mask biologically relevant signatures between different cell types. Therefore, additional studies using single-cell RNA-seq (scRNA-seq) technology will be required to overcome this limitation and determine the transcriptional program in individual cell populations including cardiomyocytes, fibroblasts, endothelial cells, and immune cells with unparalleled resolution (Farbehi et al., 2019).

Although there are plenty of studies investigating the role of the immune system in myocardial infarction (Lai et al., 2019), many aspects of immunological modulation by STAT1 and STAT3 in the infarcted heart still require further elucidation. Therefore, the overarching aim of the next experiments were to study the balance of STAT1 and STAT3 activation in murine heart endothelial cells. Several studies have reported STAT1 and STAT3 playing opposing roles in regulating multiple cellular processes including survival, proliferation, motility and inflammation (Avalle et al., 2012). In our data, we observed that upon co-stimulation with IFN- $\gamma$ and IL-6 in MHEC-5 cells, the RNA expression level of STAT1 target genes were reduced as compared to cells stimulated with IFN- $\gamma$ only, however, the phosphorylation levels of STAT1 and STAT3 were similar in both conditions.

The results presented here open up new possibilities for analyzing the role of STAT1:STAT3 heterodimers in modifying signals downstream of the receptor by integrating different signal inputs. Sadreev and colleagues recognized the importance of STAT heterodimer species in regulating immune responses (Sadreev et al., 2018). The authors postulated that the rearrangement of various STAT dimers formed by stimulation with different extracellular cytokines can regulate T-cell plasticity. They further suggested that the competing STAT complexes can indirectly inhibit each other depending on extracellular cytokine concentrations. An interesting aspect of these findings is that they highlight the biological role of STAT heterodimeric complexes which have remained enigmatic (Delgoffe and Vignali, 2013; Sadreev et al., 2018). 
The focus of my project was then directed towards studying the role of STAT1 and STAT3 proteins in controlling transendothelial migration and the regulation of the cytoskeletal ERM component ezrin in the neutrophil-like HL-60 cell line. In light of their importance in ischemic heart disease, neutrophils have been extensively studied, with excessive infiltration contributing to adverse cardiac remodeling in the injured myocardium. Accordingly, directing detrimental neutrophils away from the site of cardiac injury is an interesting avenue to investigate (de Oliveira et al., 2016).

In a parallel plate flow chamber assay, analysis of STAT1 and STAT3 knockdown HL-60 cells showed a reduced transendothelial migration across an endothelial monolayer compared to control cells. Therefore, STAT1 and STAT3 knockdown highlighted defects in neutrophils' transendothelial migration, as modelled in HL-60 cells. Our original observation indicated that ezrin expression levels are lower in STAT1 and STAT3 knockdown HL-60 cells as compared to control cells following differentiation with DMSO at day 4. The recently published paper by Ivetic et al. provided an interesting model for monocyte behavior during transendothelial migration. Herein, the authors described a novel physiological function of sequential binding of ezrin and moesin to L-selectin, which modulates L-selectin shedding and hence protrusion dynamics and polarity. In our data, it is interesting to note that STAT3 knockdown in HL-60 cells may result in disorganized cell polarity and loose directional persistence. These novel data are in agreement with earlier reports, in which STAT3 knockdown in mouse embryonic fibroblasts resulted in increased Rac1 activity, which regulates directional persistence and generation of actin stress fibers, thus inducing a random mode of migration (Teng et al., 2009). The authors have also established a direct binding of STAT3 to $\beta$-PIX, which is a known Rac1 activator, suggesting a possible mechanism for STAT3 in modulating actin cytoskeleton reorganization and inducing directional movement (Teng et al., 2009). Other studies have focused on the role of IL-6 in promoting epithelial-tomesenchymal transition and metastatic behavior via STAT3 signalling pathway within the context of carcinogenesis (Yadav et al., 2011). Nevertheless, the physiological significance of these data is yet to be evaluated within the context of myocardial infarction. These studies would pave the way for more sophisticated experiments involving the re-introduction of shRNA immune constructs of mutant STAT1-F77A. Alternatively, the more recently developed CRISPR/Cas9 platform of gene editing could be used to engineer the F77A mutant.

With recent advancements in STAT1 signalling, current concepts are continually evolving to describe the molecular and cellular processes in the failing heart. In concert with prior studies, 
the observed upregulation of immune cell markers and adhesion molecules and the significant decrease in the expression of oxidative phosphorylation genes in STAT1-F77A as compared to wild-type mice may be beneficial in the early phase to meet the demands of repair processes through metabolic rewiring and immune cells recruitment. Nowadays, a compelling body of experimental evidence indicates that innate and adaptive immune cells play a fundamental role in cardiac remodeling (Aubert et al., 2013). Certainly, our finding that immunity-related pathways are significantly upregulated following myocardial infarction is in good agreement with previous studies showing increased expression of genes involved in inflammation, chemotaxis, blood agglutination, and immunity in the myocardial tissue of MIoperated mice (Li et al., 2019). Furthermore, we observed a downregulation of a multitude of genes that are involved in ATP production when STAT1 tetramer activity is suppressed. The heart fails when its generation or utilization of free energy in the process of contraction is inadequate for the cardiac load imposed. These observations may help explain the why STAT1-F77A mice are protected from adverse cardiac remodeling. Overall, these results provide evidence for a plausible link between interferon signalling and the modulation of gene signature related to mitochondrial ATP synthesis. Our findings will encourage further investigations to study if these metabolic changes observed at the transcriptional level of the entire genome are reflected by parallel changes in the enzymatic activity of the proteins as well as the levels of metabolites. Therapeutic interventions which modify substrate utilization will be a promising target to optimize cardiac metabolism and function. Hence, investigating how does STAT signalling affect the utilization of alternative fuel sources in cardiac cells in addition to the major substrates utilized will be of major importance for an understanding of cardiac metabolic reprogramming. 


\section{References}

Abroun S, Saki N, Ahmadvand M, Asghari F, Salari F, Rahim F. STATs: an old story, yet mesmerizing. Cell J 2015;17(3):395-411.

Adams TE, Hansen JA, Starr R, Nicola NA, Hilton DJ, Billestrup N. Growth hormone preferentially induces the rapid, transient expression of SOCS-3, a novel inhibitor of cytokine receptor signaling. J Biol Chem 1998; 273(3):1285-7.

Akira S. IL-6-regulated transcription factors. Int J Biochem Cell Biol 1997;29(12):1401-18.

Akira S. Functional roles of STAT family proteins: lessons from knockout mice. Stem Cells 1999;17(3):138-46.

Akira S, Nishio Y, Inoue M, Wang XJ, Wei S, Matsusaka T, Yoshida K, Sudo T, Naruto M, Kishimoto T. Molecular cloning of APRF, a novel IFN-stimulated gene factor 3 p91-related transcription factor involved in the gp130-mediated signaling pathway. Cell 1994;77(1):6371.

Altara R, Mallat Z, Booz GW, Zouein FA. The CXCL10/CXCR3 axis and cardiac inflammation: implications for immunotherapy to treat infectious and noninfectious diseases of the heart. J Immunol Res 2016; 4396368.

Aman MJ, Leonard WJ. Cytokine signaling: cytokine-inducible signaling inhibitors. Curr Biol 1997;7(12):R784-8.

Argetsinger LS, Campbell GS, Yang X, Witthuhn BA, Silvennoinen O, Ihle JN, Carter-Su C. Identification of JAK2 as a growth hormone receptor-associated tyrosine kinase. Cell 1993;74(2):237-44.

Aubert G, Vega RB, Kelly DP. Perturbations in the gene regulatory pathways controlling mitochondrial energy production in the failing heart. Biochim Biophys Acta 2013;1833(4):840-7. 
Avalle L, Pensa S, Regis G, Novelli F, Poli V. STAT1 and STAT3 in tumorigenesis: a matter of balance. JAKSTAT 2012;1(2):65-72.

Azevedo PS, Polegato BF, Minicucci MF, Paiva SA, Zornoff LA. Cardiac remodeling: concepts, clinical impact, pathophysiological mechanisms and pharmacologic treatment. Arq Bras Cardiol 2016;106(1):62-9.

Bao J, Zervos AS. Isolation and characterization of Nmi, a novel partner of Myc proteins. Oncogene 1996;12(10):2171-6.

Barry SP, Townsend PA, Latchman DS, Stephanou A. Role of the JAK-STAT pathway in myocardial injury. Trends Mol Med 2007;13(2):82-9.

Becker S, Groner B, Müller CW. Three-dimensional structure of the Stat3 $\beta$ homodimer bound to DNA. Nature 1998;394(6689):145-51.

Begitt A, Droescher M, Meyer T, Schmid CD, Baker M, Antunes F, Knobeloch KP, Owen MR, Naumann R, Decker T, Vinkemeier U. STAT1-cooperative DNA binding distinguishes type 1 from type 2 interferon signaling. Nat Immunol 2014;15(2):168-76.

Begitt A, Meyer T, van Rossum M, Vinkemeier U. Nucleocytoplasmic translocation of Stat1 is regulated by a leucine-rich export signal in the coiled-coil domain. Proc Natl Acad Sci U S A 2000;97(19):10418-23.

Binari R, Perrimon N. Stripe-specific regulation of pair-rule genes by hopscotch, a putative Jak family tyrosine kinase in Drosophila. Genes Dev 1994;8(3):300-12.

Birkenfeld AL, Jordan J, Dworak M, Merkel T, Burnstock G. Myocardial metabolism in heart failure: purinergic signalling and other metabolic concepts. Pharmacol Ther 2019;194:132144.

Boengler K, Hilfiker-Kleiner D, Heusch G, Schulz R. Inhibition of permeability transition pore opening by mitochondrial STAT3 and its role in myocardial ischemia/reperfusion. Basic Res Cardiol 2010;105(6):771-85. 
Boisson-Dupuis S, Kong XF, Okada S, Cypowyj S, Puel A, Abel L, Casanova JL. Inborn errors of human STAT1: allelic heterogeneity governs the diversity of immunological and infectious phenotypes. Curr Opin Immunol 2012;24(4):364-78.

Bolli R, Stein AB, Guo Y, Wang OL, Rokosh G, Dawn B, Molkentin JD, Sanganalmath SK, Zhu Y, Xuan YT. A murine model of inducible, cardiac-specific deletion of STAT3: its use to determine the role of STAT3 in the upregulation of cardioprotective proteins by ischemic preconditioning. J Mol Cell Cardiol 2011;50(4):589-97.

Bousoik E, Montazeri Aliabadi H. "Do we know jack" about JAK? A closer look at JAK/STAT signaling pathway. Front Oncol 2018;8:287.

Bromberg JF, Horvath CM, Besser D, Lathem WW, Darnell JE Jr. Stat3 activation is required for cellular transformation by v-src. Mol Cell Biol 1998;18(5):2553-8.

Bronner ME, LeDouarin NM. Development and evolution of the neural crest: an overview. Dev Biol 2012;366(1):2-9.

Buck MD, Sowell RT, Kaech SM, Pearce EL. Metabolic instruction of immunity. Cell 2017;169(4):570-586.

Butler KL, Huffman LC, Koch SE, Hahn HS, Gwathmey JK. STAT-3 activation is necessary for ischemic preconditioning in hypertrophied myocardium. Am J Physiol Heart Circ Physiol 2006;291(2):H797-803.

Böhmer FD, Friedrich K. Protein tyrosine phosphatases as wardens of STAT signaling. JAKSTAT 2014;3(1):e28087.

Cao Y, Li R, Li Y, Zhang T, Wu N, Zhang J, Guo Z. Identification of transcription factorgene regulatory network in acute myocardial infarction. Heart Lung Circ 2017;26(4):343353.

Casanova JL, Holland SM, Notarangelo LD. Inborn errors of human JAKs and STATs. Immunity 2012;36(4):515-28. 
Cavalera M, Frangogiannis NG. Targeting the chemokines in cardiac repair. Curr Pharm Des 2014;20(12):1971-9.

Cavallaro U, Dejana E. Adhesion molecule signalling: not always a sticky business. Nat Rev Mol Cell Biol 2011;12(3):189-97.

Chen X, Vinkemeier U, Zhao Y, Jeruzalmi D, Darnell JE Jr, Kuriyan J. Crystal structure of a tyrosine-phosphorylated STAT-1 dimer bound to DNA. Cell 1998;93(5):827-39.

Chen Z, Liu M, Li L, Chen L. Involvement of the Warburg effect in non-tumor diseases processes. J Cell Physiol 2018;233(4):2839-2849.

Cheon H, Stark GR. Unphosphorylated STAT1 prolongs the expression of interferon-induced immune regulatory genes. Proc Natl Acad Sci U S A 2009;106(23):9373-8.

Costa-Pereira AP, Tininini S, Strobl B, Alonzi T, Schlaak JF, Is'harc H, Gesualdo I, Newman SJ, Kerr IM, Poli V. Mutational switch of an IL-6 response to an interferon- $\gamma$-like response. Proc Natl Acad Sci U S A 2002;99(12):8043-7.

Darnell JE Jr, Kerr IM, Stark GR. JAK-STAT pathways and transcriptional activation in response to IFNs and other extracellular signaling proteins. Science 1994;264(5164):1415-21.

Dawn B, Xuan YT, Guo Y, Rezazadeh A, Stein AB, Hunt G, Wu WJ, Tan W, Bolli R.IL-6 plays an obligatory role in late preconditioning via JAK-STAT signaling and upregulation of iNOS and COX-2. Cardiovasc Res 2004;64(1):61-71.

de Oliveira S, Rosowski EE, Huttenlocher A. Neutrophil migration in infection and wound repair: going forward in reverse. Nat Rev Immunol. 2016;16(6):378-91.

Delgoffe GM, Vignali DA. STAT heterodimers in immunity: A mixed message or a unique signal? JAKSTAT 2013;2(1):e23060.

Devaux Y, Azuaje F, Vausort M, Yvorra C, Wagner DR. Integrated protein network and microarray analysis to identify potential biomarkers after myocardial infarction. Funct Integr Genomics 2010;10(3):329-37. 
Dinarello CA. Historical insights into cytokines. Eur J Immunol 2007;37 Suppl 1:S34-45.

Dobin A, Davis CA, Schlesinger F, Drenkow J, Zaleski C, Jha S, Batut P, Chaisson M, Gingeras TR. STAR: ultrafast universal RNA-seq aligner. Bioinformatics 2013;29(1):15-21.

Dodington DW, Desai HR, Woo M. JAK/STAT - Emerging players in metabolism. Trends Endocrinol Metab 2018;29(1):55-65.

Doenst T, Nguyen TD, Abel ED. Cardiac metabolism in heart failure: implications beyond ATP production. Circ Res 2013;113(6):709-24.

Droescher M, Begitt A, Marg A, Zacharias M, Vinkemeier U. Cytokine-induced paracrystals prolong the activity of signal transducers and activators of transcription (STAT) and provide a model for the regulation of protein solubility by small ubiquitin-like modifier (SUMO). J Biol Chem 2011;286(21):18731-46.

Durinck S, Spellman PT, Birney E, Huber W. Mapping identifiers for the integration of genomic datasets with the R/Bioconductor package biomaRt. Nat Protoc 2009;4(8):1184-91.

Dustin ML, Chan AC. Signaling takes shape in the immune system. Cell 2000;103(2):283-94.

Dutta P, Courties G, Wei Y, Leuschner F, Gorbatov R, Robbins CS, Iwamoto Y, Thompson B, Carlson AL, Heidt T, Majmudar MD, Lasitschka F, Etzrodt M, WatermanP, Waring MT, Chicoine AT, van der Laan AM, Niessen HW, Piek JJ, Rubin BB, ButanyJ, Stone JR, Katus HA, Murphy SA, Morrow DA, Sabatine MS, Vinegoni C, MoskowitzMA, Pittet MJ, Libby P, Lin CP, Swirski FK, Weissleder R, Nahrendorf M. Myocardial infarction accelerates atherosclerosis. Nature 2012;487(7407):325-9.

El-Adawi H, Deng L, Tramontano A, Smith S, Mascareno E, Ganguly K, CastilloR, El-Sherif $\mathrm{N}$. The functional role of the JAK-STAT pathway in post-infarction remodeling. Cardiovasc Res 2003;57(1):129-38.

Epelman S, Liu PP, Mann DL. Role of innate and adaptive immune mechanisms in cardiac injury and repair. Nat Rev Immunol 2015;15(2):117-29. 
Farbehi N, Patrick R, Dorison A, Xaymardan M, Janbandhu V, Wystub-Lis K, HoJW, Nordon RE, Harvey RP. Single-cell expression profiling reveals dynamic flux of cardiac stromal, vascular and immune cells in health and injury. Elife 2019;26;8.

Finger S, Knorr M, Molitor M, Schüler R, Garlapati V, Waisman A, Brandt M, Münzel T, Bopp T, Kossmann S, Karbach S, Wenzel P. A sequential interferon $\gamma$ directed chemotactic cellular immune response determines survival and cardiac function post myocardial infarction. Cardiovasc Res 2019;5.

Firmbach-Kraft I, Byers M, Shows T, Dalla-Favera R, Krolewski JJ. TYK2, prototype of a novel class of non-receptor tyrosine kinase genes. Oncogene 1990;5(9):1329-36.

Forte E, Furtado MB, Rosenthal N. The interstitium in cardiac repair: role of the immunestromal cell interplay. Nat Rev Cardiol 2018;15(10):601-616.

Frangogiannis NG. The inflammatory response in myocardial injury, repair, and remodelling. Nat Rev Cardiol 2014;11(5):255-65.

Frangogiannis NG. The immune system and the remodeling infarcted heart: cell biological insights and therapeutic opportunities. J Cardiovasc Pharmacol 2014;63(3):185-95.

$\mathrm{Fu} \mathrm{XY}$. A transcription factor with $\mathrm{SH} 2$ and $\mathrm{SH} 3$ domains is directly activated by an interferon $\alpha$-induced cytoplasmic protein tyrosine kinase(s). Cell 1992;70(2):323-35.

Fuglesteg BN, Suleman N, Tiron C, Kanhema T, Lacerda L, Andreasen TV, SackMN, Jonassen AK, Mjøs OD, Opie LH, Lecour S. Signal transducer and activator of transcription 3 is involved in the cardioprotective signalling pathway activated by insulin therapy at reperfusion. Basic Res Cardiol 2008;103(5):444-53.

Gabay C, Kushner I. Acute-phase proteins and other systemic responses to inflammation. $N$ Engl J Med 1999;340(6):448-54.

Gentleman RC, Carey VJ, Bates DM, Bolstad B, Dettling M, Dudoit S, Ellis B, Gautier L, Ge Y, Gentry J, Hornik K, Hothorn T, Huber W, Iacus S, Irizarry R, Leisch F, Li C, Maechler M, 
Rossini AJ, Sawitzki G, Smith C, Smyth G, Tierney L, Yang JY, Zhang J. Bioconductor: open software development for computational biology and bioinformatics. Genome Biol 2004;5(10):R80.

Gomez I, Duval V, Silvestre JS. Cardiomyocytes and macrophages discourse on the method to govern cardiac repair. Front Cardiovasc Med 2018;5:134.

Goodman MD, Koch SE, Afzal MR, Butler KL. STAT subtype specificity and ischemic preconditioning in mice: is STAT-3 enough? Am J Physiol Heart Circ Physiol 2011;300(2):H522-6.

Goodman MD, Koch SE, Fuller-Bicer GA, Butler KL. Regulating RISK: a role for JAKSTAT signaling in postconditioning? Am J Physiol Heart Circ Physiol 2008;295(4):H164956.

Gordon JW, Shaw JA, Kirshenbaum LA. Multiple facets of NF- $\kappa B$ in the heart: to be or not to NF-кB. Circ Res 2011;108(9):1122-32.

Gough DJ, Corlett A, Schlessinger K, Wegrzyn J, Larner AC, Levy DE. Mitochondrial STAT3 supports Ras-dependent oncogenic transformation. Science 2009;324(5935):1713-6.

Greenlund AC, Farrar MA, Viviano BL, Schreiber RD. Ligand-induced IFN- $\gamma$ receptor tyrosine phosphorylation couples the receptor to its signal transduction system (p91). EMBO $J$ 1994;13(7):1591-600.

Gross ER, Hsu AK, Gross GJ. The JAK/STAT pathway is essential for opioid-induced cardioprotection: JAK2 as a mediator of STAT3, Akt, and GSK-3 $\beta$. Am J Physiol Heart Circ Physiol 2006;291(2):H827-34.

Heidt T, Courties G, Dutta P, Sager HB, Sebas M, Iwamoto Y, Sun Y, Da Silva N, Panizzi P, van der Laan AM, Swirski FK, Weissleder R, Nahrendorf M. Differential contribution of monocytes to heart macrophages in steady-state and after myocardial infarction. Circ Res 2014;115(2):284-95. 
Heim MH, Kerr IM, Stark GR, Darnell JE Jr. Contribution of STAT SH2 groups to specific interferon signaling by the Jak-STAT pathway. Science 1995;267(5202):1347-9.

Heusch G, Musiolik J, Kottenberg E, Peters J, Jakob H, Thielmann M. STAT5 activation and cardioprotection by remote ischemic preconditioning in humans: short communication. Circ Res 2012;110(1):111-5.

Heusch G, Musiolik J, Gedik N, Skyschally A. Mitochondrial STAT3 activation and cardioprotection by ischemic postconditioning in pigs with regional myocardial ischemia/reperfusion. Circ Res 2011;109(11):1302-8.

Hilfiker-Kleiner D, Hilfiker A, Fuchs M, Kaminski K, Schaefer A, SchiefferB, Hillmer A, Schmiedl A, Ding Z, Podewski E, Podewski E, Poli V, Schneider MD, Schulz R, Park JK, Wollert KC, Drexler H. Signal transducer and activator of transcription 3 is required for myocardial capillary growth, control of interstitial matrix deposition, and heart protection from ischemic injury. Circ Res 2004;95(2):187-95.

Ho J, Pelzel C, Begitt A, Mee M, Elsheikha HM, Scott DJ, Vinkemeier U. STAT2 is a pervasive cytokine regulator due to its inhibition of STAT1 in multiple signaling pathways. PLoS Biol 2016;14(10):e2000117.

Horvath CM, Wen Z, Darnell JE Jr. A STAT protein domain that determines DNA sequence recognition suggests a novel DNA-binding domain. Genes Dev 1995;9(8):984-94.

$\mathrm{Hu}$ Y, Hu X, Boumsell L, Ivashkiv LB. IFN- $\gamma$ and STAT1 arrest monocyte migration and modulate RAC/CDC42 pathways. J Immunol 2008;180(12):8057-65.

Hüntelmann B, Staab J, Herrmann-Lingen C, Meyer T. A conserved motif in the linker domain of STAT1 transcription factor is required for both recognition and re-lease from highaffinity DNA-binding sites, Plos One 2014:9:e97633

Huffman LC, Koch SE, Butler KL. Coronary effluent from a preconditioned heart activates the JAK-STAT pathway and induces cardioprotection in a donor heart. Am J Physiol Heart Circ Physiol 2008;294(1):H257-62. 
Ihle JN. STATs: signal transducers and activators of transcription. Cell 1996;84(3):331-4.

Isaacs A, Lindenmann J. Virus interference. I. The interferon. Proc R Soc Lond B Biol Sci 1957;147(927):258-67.

Jacoby JJ, Kalinowski A, Liu MG, Zhang SS, Gao Q, Chai GX, Ji L, Iwamoto Y, Li E, Schneider M, Russell KS, Fu XY. Cardiomyocyte-restricted knockout of STAT3 results in higher sensitivity to inflammation, cardiac fibrosis, and heart failure with advanced age. Proc Natl Acad Sci U S A 2003;100(22):12929-34.

Jug B, Salobir BG, Vene N, Sebestjen M, Sabovic M, Keber I. Interleukin-6 is a stronger prognostic predictor than high-sensitive $\mathrm{C}$-reactive protein in patients with chronic stable heart failure. Heart Vessels 2009;24(4):271-6.

Jung M, Ma Y, Iyer RP, DeLeon-Pennell KY, Yabluchanskiy A, Garrett MR, Lindsey ML. IL-10 improves cardiac remodeling after myocardial infarction by stimulating M2 macrophage polarization and fibroblast activation. Basic Res Cardiol 2017;112(3):33.

Kanehisa M, Goto S. KEGG: kyoto encyclopedia of genes and genomes. Nucleic Acids Res 2000;28(1):27-30.

Kawata T, Shevchenko A, Fukuzawa M, Jermyn KA, Totty NF, Zhukovskaya NV, Sterling AE, Mann M, Williams JG. SH2 signaling in a lower eukaryote: a STAT protein that regulates stalk cell differentiation in dictyostelium. Cell 1997;89(6):909-16.

King KR, Aguirre AD, Ye YX, Sun Y, Roh JD, Ng RP Jr, Kohler RH, ArlauckasSP, Iwamoto Y, Savol A, Sadreyev RI, Kelly M, Fitzgibbons TP, Fitzgerald KA, Mitchison T, Libby P, Nahrendorf M, Weissleder R. IRF3 and type I interferons fuel a fatal response to myocardial infarction. Nat Med 2017;23(12):1481-1487.

Kisseleva T, Bhattacharya S, Braunstein J, Schindler CW. Signaling through the JAK/STAT pathway, recent advances and future challenges. Gene 2002;285(1-2):1-24. Review. 
Kodama H, Fukuda K, Pan J, Makino S, Sano M, Takahashi T, Hori S, Ogawa S. Biphasic activation of the JAK/STAT pathway by angiotensin II in rat cardiomyocytes. Circ Res 1998;82(2):244-50.

Kontou P, Pavlopoulou A, Braliou G, Bogiatzi S, Dimou N, Bangalore S, Bagos P. Identification of gene expression profiles in myocardial infarction: a systematic review and meta-analysis. BMC Med Genomics 2018;11(1):109.

Kung G, Konstantinidis K, Kitsis RN. Programmed necrosis, not apoptosis, in the heart. Circ Res 2011;108(8):1017-36.

Kunisada K, Negoro S, Tone E, Funamoto M, Osugi T, Yamada S, Okabe M, Kishimoto T, Yamauchi-Takihara K. Signal transducer and activator of transcription 3 in the heart transduces not only a hypertrophic signal but a protective signal against doxorubicin-induced cardiomyopathy. Proc Natl Acad Sci U S A 2000;97(1):315-9.

Lai SL, Marín-Juez R, Stainier DYR. Immune responses in cardiac repair and regeneration: a comparative point of view. Cell Mol Life Sci 2019;76(7):1365-1380.

Lambert JM, Lopez EF, Lindsey ML. Macrophage roles following myocardial infarction. Int J Cardiol 2008;130(2):147-58.

Lamouille S, Xu J, Derynck R. Molecular mechanisms of epithelial-mesenchymal transition. Nat Rev Mol Cell Biol 2014;15(3):178-96.

Lee AJ, Ashkar AA. The dual nature of type I and type II interferons. Front Immunol 2018;9:2061.

Leocádio PCL, Menta PLDR, Dias MTS, Fraga JR, Goulart AC, Santos IS, Lotufo PA, Bensenor IM, Alvarez-Leite JI. Low serum levels of CCL2 are associated with worse prognosis in patients with acute coronary syndrome: 2-year survival analysis. Biomed Pharmacother 2019;109:1411-1416. 
Levy DE, Darnell JE Jr. Interferon-dependent transcriptional activation: signal transduction without second messenger involvement? New Biol 1990;2(10):923-8.

Levy DE, Darnell JE Jr. Stats: transcriptional control and biological impact. Nat Rev Mol Cell Biol 2002;3(9):651-62.

Li Y, Wang C, Li T, Ma L, Fan F, Jin Y, Shen J. The whole transcriptome and proteome changes in the early stage of myocardial infarction. Cell Death Discov 2019;5:73.

Liao Y, Smyth GK, Shi W. featureCounts: an efficient general purpose program for assigning sequence reads to genomic features. Bioinformatics 2014;30(7):923-30.

Lin RC, Weeks KL, Gao XM, Williams RB, Bernardo BC, Kiriazis H, Matthews VB,Woodcock EA, Bouwman RD, Mollica JP, Speirs HJ, Dawes IW, Daly RJ, Shioi T, IzumoS, Febbraio MA, Du XJ, McMullen JR. PI3K (p110 $\alpha$ ) protects against myocardial infarction-induced heart failure: identification of PI3K-regulated miRNA and mRNA. Arterioscler Thromb Vasc Biol 2010;30(4):724-32.

Lionetti V, Stanley WC, Recchia FA. Modulating fatty acid oxidation in heart failure. Cardiovasc Res 2011;90(2):202-9.

Liu Y, Morley M, Brandimarto J, Hannenhalli S, Hu Y, Ashley EA, Tang WH, Moravec CS, Margulies KB, Cappola TP, Li M; MAGNet consortium. RNA-seq identifies novel myocardial gene expression signatures of heart failure. Genomics 2015;105(2):83-9.

Love MI, Huber W, Anders S. Moderated estimation of fold change anddispersion for RNAseq data with DESeq2. Genome Biol 2014;15(12):550.

Majoros A, Platanitis E, Kernbauer-Hölzl E, Rosebrock F, Müller M, Decker T. Canonical and non-canonical aspects of JAK-STAT signaling: lessons from interferons for cytokine responses. Front Immunol 2017;26;8:29. 
Mascareno E, El-Shafei M, Maulik N, Sato M, Guo Y, Das DK, Siddiqui MA. JAK/STAT signaling is associated with cardiac dysfunction during ischemia and reperfusion. Circulation 2001;104(3):325-9.

Massa M, Rosti V, Ferrario M, Campanelli R, Ramajoli I, Rosso R, De Ferrari GM, Ferlini M, Goffredo L, Bertoletti A, Klersy C, Pecci A, Moratti R, Tavazzi L. Increased circulating hematopoietic and endothelial progenitor cells in the early phase of acute myocardial infarction. Blood 2005;105(1):199-206.

McBride KM, Reich NC. The ins and outs of STAT1 nuclear transport. Sci STKE 2003;2003(195):RE13.

McCormick J, Suleman N, Scarabelli TM, Knight RA, Latchman DS, Stephanou A. STAT1 deficiency in the heart protects against myocardial infarction by enhancing autophagy. $J$ Cell Mol Med 2012;16(2):386-93.

Meng X, Yang J, Dong M, Zhang K, Tu E, Gao Q, Chen W, Zhang C, Zhang Y. Regulatory T cells in cardiovascular diseases. Nat Rev Cardiol 2016;13(3):167-79.

Mertens C, Zhong M, Krishnaraj R, Zou W, Chen X, Darnell JE Jr. Dephosphorylation of phosphotyrosine on STAT1 dimers requires extensive spatial reorientation of the monomers facilitated by the N-terminal domain. Genes Dev 2006;20(24):3372-81.

Mesev EV, LeDesma RA, Ploss A. Decoding type I and III interferon signaling during viral infection. Nat Microbiol 2019.

Meyer T, Voigt N. In search for novel functions of adenosine 5'-triphosphate (ATP) in the heart. Cardiovasc Res 2017;113(14):e59-e60.

Mouton AJ, DeLeon-Pennell KY, Rivera Gonzalez OJ, Flynn ER, Freeman TC, Saucerman JJ, Garrett MR, Ma Y, Harmancey R, Lindsey ML. Mapping macrophage polarization over the myocardial infarction time continuum. Basic Res Cardiol 2018;113(4):26. 
Muller O, Delrue L, Hamilos M, Vercauteren S, Ntalianis A, Trana C, Mangiacapra F, Dierickx K, De Bruyne B, Wijns W, Behfar A, Barbato E, Terzic A, Vanderheyden M, Bartunek J. Transcriptional fingerprint of human whole blood at the site of coronary occlusion in acute myocardial infarction. Euro Intervention 2011;7(4):458-66.

Muller WA. The regulation of transendothelial migration: new knowledge and new questions. Cardiovasc Res 2015;107(3):310-20.

Murray NE, Gann A. What has phage lambda ever done for us? Curr Biol 2007;17(9):R30512.

Nagoshi T, Yoshimura M, Rosano GM, Lopaschuk GD, Mochizuki S. Optimization of cardiac metabolism in heart failure. Curr Pharm Des 2011;17(35):3846-53. Review.

Nahrendorf M, Swirski FK. Innate immune cells in ischaemic heart disease: does myocardial infarction beget myocardial infarction? Eur Heart J 2016;37(11):868-72.

Nahrendorf M, Swirski FK. Monocyte and macrophage heterogeneity in the heart. Circ Res 2013;112(12):1624-33.

Nahrendorf M, Swirski FK, Aikawa E, Stangenberg L, Wurdinger T, FigueiredoJL, Libby P, Weissleder R, Pittet MJ. The healing myocardium sequentially mobilizes two monocyte subsets with divergent and complementary functions. J Exp Med 2007;204(12):3037-47.

Nieto M, Frade JM, Sancho D, Mellado M, Martinez-A C, Sánchez-Madrid F. Polarization of chemokine receptors to the leading edge during lymphocyte chemotaxis. J Exp Med 1997;186(1):153-8.

Nojiri H, Shimizu T, Funakoshi M, Yamaguchi O, Zhou H, Kawakami S, Ohta Y, Sami M, Tachibana T, Ishikawa H, Kurosawa H, Kahn RC, Otsu K, Shirasawa T. Oxidative stress causes heart failure with impaired mitochondrial respiration. $J$ Biol Chem 2006;281(44):33789-801. 
Noordali H, Loudon BL, Frenneaux MP, Madhani M. Cardiac metabolism - a promising therapeutic target for heart failure. Pharmacol Ther 2018;182:95-114.

Ortiz-Muñoz G, Martin-Ventura JL, Hernandez-Vargas P, Mallavia B, Lopez-Parra V, Lopez-Franco O, Muñoz-Garcia B, Fernandez-Vizarra P, Ortega L, Egido J, Gomez-Guerrero C. Suppressors of cytokine signaling modulate JAK/STAT-mediated cell responses during atherosclerosis. Arterioscler Thromb Vasc Biol 2009;29(4):525-31.

Oshima Y, Fujio Y, Nakanishi T, Itoh N, Yamamoto Y, Negoro S, Tanaka K, Kishimoto T, Kawase I, Azuma J. STAT3 mediates cardioprotection against ischemia/reperfusion injury through metallothionein induction in the heart. Cardiovasc Res 2005;65(2):428-35.

Parissis JT, Adamopoulos S, Venetsanou K, Kostakis G, Rigas A, Karas SM, Kremastinos D. Plasma profiles of circulating granulocyte-macrophage colony-stimulating factor and soluble cellular adhesion molecules in acute myocardial infarction. Contribution to post-infarction left ventricular dysfunction. Eur Cytokine Netw 2004;15(2):139-44.

Pedretti S, Raddatz E. STAT3 $\alpha$ interacts with nuclear GSK3 $\beta$ and cytoplasmic RISK pathway and stabilizes rhythm in the anoxic-reoxygenated embryonic heart. Basic Res Cardiol 2011;106(3):355-69.

Pestka S. The interferons: 50 years after their discovery, there is much more to learn. $J$ Biol Chem 2007;282(28):20047-51.

Phillips D, Reilley MJ, Aponte AM, Wang G, Boja E, Gucek M, Balaban RS. Stoichiometry of STAT3 and mitochondrial proteins: implications for the regulation of oxidative phosphorylation by protein-protein interactions. J BiolChem 2010;285(31):23532-6.

Pinto AR, Paolicelli R, Salimova E, Gospocic J, Slonimsky E, Bilbao-CortesD, Godwin JW, Rosenthal NA. An abundant tissue macrophage population in the adult murine heart with a distinct alternatively-activated macrophage profile. PLoSOne 2012;7(5):e36814.

Pitroda SP, Wakim BT, Sood RF, Beveridge MG, Beckett MA, MacDermed DM, Weichselbaum RR, Khodarev NN. STAT1-dependent expression of energy metabolic 
pathways links tumour growth and radioresistance to the Warburg effect. BMC Med 2009;7:68.

Prabhu SD, Frangogiannis NG. The biological basis for cardiac repair after myocardial infarction: from inflammation to fibrosis. Circ Res 2016;119(1):91-112.

Randolph GJ. Dendritic cell migration to lymph nodes: cytokines, chemokines, and lipid mediators. Semin Immunol 2001;13(5):267-74.

Remels AHV, Derks WJA, Cillero-Pastor B, Verhees KJP, Kelders MC, HeggermontW, Carai P, Summer G, Ellis SR, de Theije CC, Heeren RMA, Heymans S, Papageorgiou AP, van Bilsen M. NF- $\kappa$ B-mediated metabolic remodelling in the inflamed heart in acute viral myocarditis. Biochim Biophys Acta Mol Basis Dis 2018;1864(8):2579-2589.

Rumalla VK, Calvano SE, Spotnitz AJ, Krause TJ, Hilkert RJ, Lin E, Lowry SF. Alterations in immunocyte tumor necrosis factor receptor and apoptosis in patients with congestive heart failure. Ann Surg 2002;236(2):254-60.

Ruparelia N, Godec J, Lee R, Chai JT, Dall'Armellina E, McAndrew D, DigbyJE, Forfar JC, Prendergast BD, Kharbanda RK, Banning AP, Neubauer S, Lygate CA, Channon KM, Haining NW, Choudhury RP. Acute myocardial infarction activates distinct inflammation and proliferation pathways in circulating monocytes, prior to recruitment, and identified through conserved transcriptional responses in mice and humans. Eur Heart J 2015;36(29):1923-34.

Ruppert V, Meyer T. JAK-STAT signaling circuits in myocarditis and dilated cardiomyopathy. Herz 2007;32(6):474-81. Review.

Sadreev II, Chen MZQ, Umezawa Y, Biktashev VN, Kemper C, Salakhieva DV, Welsh GI, Kotov NV. The competitive nature of signal transducer and activator of transcription complex formation drives phenotype switching of T cells. Immunology 2018;153(4):488-501.

Saparov A, Ogay V, Nurgozhin T, Chen WCW, Mansurov N, Issabekova A, Zhakupova J. Role of the immune system in cardiac tissue damage and repair following myocardial infarction. Inflamm Res 2017;66(9):739-751. 
Satoh J, Tabunoki H. A comprehensive profile of Chip-Seq-based stat1 target genes suggests the complexity of statl-mediated gene regulatory mechanisms. Gene Regul Syst Bio 2013;7:41-56.

Satou R, Gonzalez-Villalobos RA. JAK-STAT and the renin-angiotensin system: The role of the JAK-STAT pathway in blood pressure and intrarenal renin-angiotensin system regulation. JAKSTAT 2012;1(4):250-6.

Schirone L, Forte M, Palmerio S, Yee D, Nocella C, Angelini F, Pagano F,Schiavon S, Bordin A, Carrizzo A, Vecchione C, Valenti V, Chimenti I, De Falco E, Sciarretta S, Frati G. A review of the molecular mechanisms underlying the development and progression of cardiac remodeling. Oxid Med Cell Longev 2017;2017:3920195.

Semenza GL. Hypoxia-inducible factor 1 and cardiovascular disease. Annu Rev Physiol 2014;76:39-56.

Shuai K. Regulation of cytokine signaling pathways by PIAS proteins. Cell Res. 2006;16(2):196-202.

Simon AR, Rai U, Fanburg BL, Cochran BH. Activation of the JAK-STAT pathway by reactive oxygen species. Am J Physiol 1998;275(6):C1640-52.

Smith CC, Dixon RA, Wynne AM, Theodorou L, Ong SG, Subrayan S, Davidson SM, Hausenloy DJ, Yellon DM. Leptin-induced cardioprotection involves JAK/STAT signaling that may be linked to the mitochondrial permeability transition pore. Am J Physiol Heart Circ Physiol 2010;299(4):H1265-70.

Smith RM, Suleman N, Lacerda L, Opie LH, Akira S, Chien KR, Sack MN. Genetic depletion of cardiac myocyte STAT-3 abolishes classical preconditioning. Cardiovasc Res 2004;63(4):611-6.

Staab J, Herrmann-Lingen C, Meyer T. A rapid conformational rearrangement of STAT1 dimers is required for termination rather than for amplification of interferon- $\gamma$ signaling. JAKSTAT 2013;2(1):e23576. 
Stanton LW, Garrard LJ, Damm D, Garrick BL, Lam A, Kapoun AM, Zheng Q, Protter AA, Schreiner GF, White RT. Altered patterns of gene expression in response to myocardial infarction. Circ Res 2000;86(9):939-45.

Stephanou A, Scarabelli TM, Brar BK, Nakanishi Y, Matsumura M, Knight RA, Latchman DS. Induction of apoptosis and Fas receptor/Fas ligand expression by ischemia/reperfusion in cardiac myocytes requires serine 727 of the STAT-1 transcription factor but not tyrosine 701 . J Biol Chem 2001;276(30):28340-7.

Stephanou A, Brar BK, Scarabelli TM, Jonassen AK, Yellon DM, Marber MS, Knight RA, Latchman DS. Ischemia-induced STAT-1 expression and activation play a critical role in cardiomyocyte apoptosis. J Biol Chem 2000;275(14):10002-8.

Stumpf C, Seybold K, Petzi S, Wasmeier G, Raaz D, Yilmaz A, Anger T, Daniel WG, Garlichs CD. Interleukin-10 improves left ventricular function in rats with heart failure subsequent to myocardial infarction. Eur J Heart Fail 2008;10(8):733-9.

Takeda K, Noguchi K, Shi W, Tanaka T, Matsumoto M, Yoshida N, Kishimoto T, Akira S. Targeted disruption of the mouse $q$ gene leads to early embryonic lethality. Proc Natl Acad Sci U S A 1997;94(8):3801-4.

Tanaka T, Soriano MA, Grusby MJ. SLIM is a nuclear ubiquitin E3 ligase that negatively regulates STAT signaling. Immunity 2005;22(6):729-36.

ten Hoeve J, de Jesus Ibarra-Sanchez M, Fu Y, Zhu W, Tremblay M, David M, Shuai K. Identification of a nuclear Stat1 protein tyrosine phosphatase. Mol Cell Biol 2002;22(16):5662-8.

Teng TS, Lin B, Manser E, Ng DC, Cao X. Stat3 promotes directional cell migration by regulating Rac1 activity via its activator $\beta$-PIX. J Cell Sci 2009;122(Pt 22):4150-9.

Thygesen K, Alpert JS, Jaffe AS, Chaitman BR, Bax JJ, Morrow DA, White HD; Executive Group on behalf of the Joint European Society of Cardiology (ESC)/American College of Cardiology (ACC)/American Heart Association (AHA)/World Heart Federation (WHF) Task 
Force for the Universal Definition of Myocardial Infarction. Fourth universal definition of myocardial infarction (2018). Circulation 2018;138(20):e618-e651.

Townsend PA, Scarabelli TM, Davidson SM, Knight RA, Latchman DS, Stephanou A. STAT-1 interacts with p53 to enhance DNA damage-induced apoptosis. J Biol Chem 2004;279(7):5811-20.

Ueland T, Gullestad L, Nymo SH, Yndestad A, Aukrust P, Askevold ET. Inflammatory cytokines as biomarkers in heart failure. Clin Chim Acta 2015;443:71-7.

van Bilsen M, Smeets PJ, Gilde AJ, van der Vusse GJ. Metabolic remodeling of the failing heart: the cardiac burn-out syndrome? Cardiovasc Res 2004;61(2):218-26.

VanDeusen JB, Shah MH, Becknell B, Blaser BW, Ferketich AK, Nuovo GJ, Ahmer BM, Durbin J, Caligiuri MA. STAT-1-mediated repression of monocyte interleukin-10 gene expression in vivo. Eur J Immunol 2006;36(3):623-30.

Varinou L, Ramsauer K, Karaghiosoff M, Kolbe T, Pfeffer K, Müller M, Decker T. Phosphorylation of the Stat1 transactivation domain is required for full-fledged IFN- $\gamma$ dependent innate immunity. Immunity 2003;19(6):793-802.

Velazquez L, Fellous M, Stark GR, Pellegrini S. A protein tyrosine kinase in the interferon $\alpha / \beta$ signaling pathway. Cell 1992;70(2):313-22.

Vinkemeier U, Moarefi I, Darnell JE Jr, Kuriyan J. Structure of the amino-terminal protein interaction domain of STAT-4. Science 1998;279(5353):1048-52.

Vultur A, Arulanandam R, Turkson J, Niu G, Jove R, Raptis L. Stat3 is required for full neoplastic transformation by the Simian Virus 40 large tumor antigen. Mol Biol Cell 2005;16(8):3832-46.

Wagner MA, Siddiqui MA. The JAK-STAT pathway in hypertrophic stress signaling and genomic stress response. JAKSTAT 2012;1(2):131-41. 
Wang J, Seo MJ, Deci MB, Weil BR, Canty JM, Nguyen J. Effect of CCR2 inhibitor-loaded lipid micelles on inflammatory cell migration and cardiac function after myocardial infarction. Int J Nanomedicine 2018;13:6441-6451.

Wang X, Guo Z, Ding Z, Mehta JL. Inflammation, autophagy, and apoptosis after myocardial infarction. J Am Heart Assoc 2018;7(9).

Wang Y, Levy DE. Comparative evolutionary genomics of the STAT family of transcription factors. JAKSTAT 2012;1(1):23-33.

Wang Y, Levy DE. C. elegans STAT: evolution of a regulatory switch. FASEB $J$ 2006;20(10):1641-52.

Wang Z, Gerstein M, Snyder M. RNA-seq: a revolutionary tool for transcriptomics. Nat Rev Genet 2009;10(1):57-63.

Wegenka UM, Buschmann J, Lütticken C, Heinrich PC, Horn F. Acute-phase response factor, a nuclear factor binding to acute-phase response elements, is rapidly activated by interleukin6 at the posttranslational level. Mol Cell Biol 1993;13(1):276-88.

Wegrzyn J, Potla R, Chwae YJ, Sepuri NB, Zhang Q, Koeck T, Derecka M, Szczepanek K, Szelag M, Gornicka A, Moh A, Moghaddas S, Chen Q, Bobbili S, Cichy J, Dulak J, Baker DP, Wolfman A, Stuehr D, Hassan MO, Fu XY, Avadhani N, DrakeJI, Fawcett P, Lesnefsky EJ, Larner AC. Function of mitochondrial Stat3 in cellular respiration. Science 2009;323(5915):793-7.

Wende AR, Brahma MK, McGinnis GR, Young ME. Metabolic origins of heart failure. JACC Basic Transl Sci 2017;2(3):297-310.

Wenta N, Strauss H, Meyer S, Vinkemeier U. Tyrosine phosphorylation regulates the partitioning of STAT1 between different dimer conformations. Proc Natl Acad Sci U S A 2008;105(27):9238-43. 
Wilks AF, Harpur AG, Kurban RR, Ralph SJ, Zürcher G, Ziemiecki A. Two novel proteintyrosine kinases, each with a second phosphotransferase-related catalytic domain, define a new class of protein kinase. Mol Cell Biol 1991;11(4):2057-65.

Witthuhn BA, Quelle FW, Silvennoinen O, Yi T, Tang B, Miura O, Ihle JN. JAK2 associates with the erythropoietin receptor and is tyrosine phosphorylated and activated following stimulation with erythropoietin. Cell 1993;74(2):227-36.

Wojciak JM, Martinez-Yamout MA, Dyson HJ, Wright PE. Structural basis for recruitment of $\mathrm{CBP} / \mathrm{p} 300$ coactivators by STAT1 and STAT2 transactivation domains. EMBO $J$ 2009;28(7):948-58.

Xia Y, Frangogiannis NG. MCP-1/CCL2 as a therapeutic target in myocardial infarction and ischemic cardiomyopathy. Inflamm Allergy Drug Targets 2007;6(2):101-7.

Xie B, Zhao J, Kitagawa M, Durbin J, Madri JA, Guan JL, Fu XY. Focal adhesion kinase activates Stat 1 in integrin-mediated cell migration and adhesion. $J$ Biol Chem 2001;276(22):19512-23.

$\mathrm{Xu}$ J, Nie HG, Zhang XD, Tian Y, Yu B. Down-regulated energy metabolism genes associated with mitochondria oxidative phosphorylation and fatty acid metabolism in viral cardiomyopathy mouse heart. Mol Biol Rep 2011;38(6):4007-13.

$\mathrm{Xu} \mathrm{X}$, Sun YL, Hoey T. Cooperative DNA binding and sequence-selective recognition conferred by the STAT amino-terminal domain. Science 1996;273(5276):794-7.

Xuan YT, Guo Y, Zhu Y, Wang OL, Rokosh G, Messing RO, Bolli R. Role of the protein kinase C-epsilon-Raf-1-MEK-1/2-p44/42 MAPK signaling cascade in the activation of signal transducers and activators of transcription 1 and 3 and induction of cyclooxygenase- 2 after ischemic preconditioning. Circulation 2005;112(13):1971-8.

Yadav A, Kumar B, Datta J, Teknos TN, Kumar P. IL-6 promotes head and neck tumor metastasis by inducing epithelial-mesenchymal transition via theJAK-STAT3-SNAIL signaling pathway. Mol Cancer Res 2011;9(12):1658-67. 
Yang B, Akhter S, Chaudhuri A, Kanmogne GD. HIV-1 gp120 induces cytokine expression, leukocyte adhesion, and transmigration across the blood-brain barrier: modulatory effects of STAT1 signaling. Microvasc Res 2009;77(2):212-9.

Yang E, Henriksen MA, Schaefer O, Zakharova N, Darnell JE Jr. Dissociation time from DNA determines transcriptional function in a STAT1 linker mutant. J Biol Chem 2002;277(16):13455-62.

Yang L, Gregorich ZR, Cai W, Zhang P, Young B, Gu Y, Zhang J, Ge Y. Quantitative proteomics and immunohistochemistry reveal insights into cellular and molecular processes in the infarct border zone one month after myocardial infarction. J Proteome Res 2017;16(5):2101-2112.

Yao K, Chen Q, Wu Y, Liu F, Chen X, Zhang Y. Unphosphorylated STAT1 represses apoptosis in macrophages during Mycobacterium tuberculosis infection. J Cell Sci 2017;130(10):1740-1751.

Yeh HH, Giri R, Chang TY, Chou CY, Su WC, Liu HS. Ha-ras oncogene-induced Stat3 phosphorylation enhances oncogenicity of the cell. DNA Cell Biol 2009;28(3):131-9.

Yu H, Pardoll D, Jove R. STATs in cancer inflammation and immunity: a leading role for STAT3. Nat Rev Cancer 2009;9(11):798-809.

Zeidler MP, Bach EA, Perrimon N. The roles of the Drosophila JAK/STAT pathway. Oncogene 2000;19(21):2598-606.

Zhang JJ, Vinkemeier U, Gu W, Chakravarti D, Horvath CM, Darnell JE Jr. Two contact regions between Stat1 and $\mathrm{CBP} / \mathrm{p} 300$ in interferon- $\gamma$ signaling. Proc Natl Acad Sci U S A 1996;93(26):15092-6.

Zhang S, Liu X, Goldstein S, Li Y, Ge J, He B, Fei X, Wang Z, Ruiz G. Role of the JAK/STAT signaling pathway in the pathogenesis of acute myocardial infarction in rats and its effect on NF-кB expression. Mol Med Rep 2013;7(1):93-8. 
Zhao Q, Wu K, Li N, Li Z, Jin F. Identification of potentially relevant genes for myocardial infarction using RNA sequencing data analysis. Exp Ther Med 2018;15(2):1456-1464.

Zhong M, Henriksen MA, Takeuchi K, Schaefer O, Liu B, ten Hoeve J, Ren Z,Mao X, Chen X, Shuai K, Darnell JE Jr. Implications of an antiparallel dimeric structure of nonphosphorylated STAT1 for the activation-inactivation cycle. Proc Natl Acad Sci U S A 2005;102(11):3966-71.

Zhong Z, Wen Z, Darnell JE Jr. Stat3: a STAT family member activated by tyrosine phosphorylation in response to epidermal growth factor and interleukin-6. Science 1994;264(5155):95-8.

Zhu X, Wen Z, Xu LZ, Darnell JE Jr. Stat 1 serine phosphorylation occurs independently of tyrosine phosphorylation and requires an activated Jak2 kinase. Mol Cell Biol 1997;17(11):6618-23.

Zhuo C, Wang Y, Wang X, Wang Y, Chen Y. Cardioprotection by ischemic postconditioning is abolished in depressed rats: role of Akt and signal transducer and activator of transcription3. Mol Cell Biochem 2011;346(1-2):39-47.

Zouein FA, Altara R, Chen Q, Lesnefsky EJ, Kurdi M, Booz GW. Pivotal importance of STAT3 in protecting the heart from acute and chronic stress: new advancement and unresolved issues. Front Cardiovasc Med 2015;30;2:36.

Zweemer AJ, Toraskar J, Heitman LH, IJzerman AP. Bias in chemokine receptor signalling. Trends Immunol 2014;35(6):243-52. 


\section{Biography}

Asmma Doudin was born in 1991 in Amman, Jordan. She received her secondary education in Fatima Al-Zahra Primary and Secondary School in Sharjah. She graduated and received her bachelor's degree in Applied Biology/Biotechnology (2012) from the College of Science and her Master's in Molecular Medicine (2014) from the College of Medicine at the University of Sharjah. Following her graduation Asmma worked as a research assistant at Sharjah Institute of Medical Research (2015). In the year 2016, she joined the International Research Training Group 1816 which is funded by the German Research Fondation. The experimental work for her Ph.D. thesis was conducted in the Molecular Psychocardiology Laboratory at the Department of Psychosomatic Medicine at Georg-August University of Göttingen, Germany, and at the Cardiovascular Department at King's College London, UK. Asmma was involved in research projects studying the molecular actions of STAT transcription factors in the pathophysiology of sterile inflammatory diseases, including murine myocardial infarction and experimental autoimmune encephalitis. She is also a young researcher in the German Centre for Cardiovascular Research (DZHK) and has published papers on the relationship between serum 25-hydroxyvitamin D and red blood cell indices in German adolescents. 